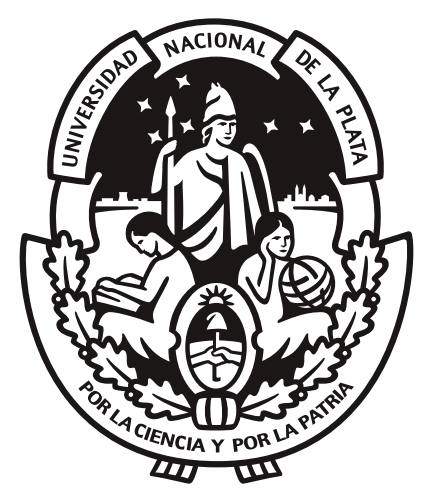

Universidad Nacional de La Plata

Facultad de Ciencias Exactas

Departamento De Física

Trabajo de Tesis Doctoral

\title{
Búsqueda de Supersimetría en el detector ATLAS (CERN-LHC)
}

Tesista: Lic. Joaquín Hoya

Director: Dr. Hernán Wahlberg 



\title{
Búsqueda de Supersimetría en el detector ATLAS (CERN-LHC)
}

\author{
Joaquín Hoya
}

\begin{abstract}
Resumen
La búsqueda de nueva física más allá del Modelo Estándar es el principal objetivo de la física experimental de altas energías desde el descubrimiento del bosón de Higgs por ATLAS y CMS en 2012. Motivaciones relacionadas con mediciones astrofísicas que predicen la existencia de materia y energía oscura, o el supuesto de una gran unificación de las fuerzas elementales de la naturaleza, entre tantas otras, justifican dicha búsqueda. La Supersimetría (SUSY) es la teoría mejor fundamentada desde el punto de vista teórico y provee modelos con estados finales atractivos para su búsqueda en colisionadores como el LHC.

En esta tesis se presenta un estudio para la búsqueda de nueva física en el marco de la teoría SUSY en estados finales con fotones aislados, jets y alto momento transversal faltante en colisiones protón-protón a una energía de centro de masa de $13 \mathrm{TeV}$. La búsqueda se realiza utilizando el conjunto de datos colectados con el detector ATLAS en el LHC entre 2015 y 2018, correspondiente a una luminosidad integrada de 139 $\mathrm{fb}^{-1}$. Para un correcto análisis de los datos es imprescindible estudiar las eficiencias de selección de objetos a medida que se adquieren datos. En este contexto, esta tesis presenta un estudio detallado del cálculo de las eficiencias del trigger de fotones y de multijets. Se presenta además un estudio de factibilidad para una búsqueda en el marco de un modelo de SUSY más complejo, conocido como Stealth SUSY, con fotones, múltiples jets y bajo momento transverso faltante en el estado final.
\end{abstract}




\title{
Search for Supersymmetry in the ATLAS detector (CERN-LHC)
}

\author{
Joaquín Hoya
}

\begin{abstract}
The search for new physics beyond the Standard Model is the main objective of experimental high energy physics since the discovery of the Higgs boson by ATLAS and CMS in 2012. Motivations related to astrophysical measurements that predict the existence of matter and dark energy, or the assumption of a great unification of the elemental forces of nature, among many others, justify this search. Supersymmetry (SUSY) is the theory best motivated from the theoretical point of view and provides models with attractive final states for their search in colliders such as the LHC.

In this thesis a study for the search of new physics within the framework of the SUSY theory in final states with isolated photons, jets and high missing transverse momentum in proton-proton collisions at a center of mass energy of $13 \mathrm{TeV}$, is presented. The search is performed using the dataset collected with the ATLAS detector at the LHC between 2015 and 2018, corresponding to an integrated luminosity of $139 \mathrm{fb}^{-1}$. For a correct analysis of the data it is essential to study the efficiencies of the object selection as data is acquired. In this context, this thesis presents a detailed study of the calculation of photon and multijet trigger efficiencies. A feasibility study is also presented for a search within the framework of a more complex SUSY model, known as Stealth SUSY, with photons, multiple jets and low missing transverse momentum in the final state.
\end{abstract}




\section{Publicaciones científicas}

Como miembro de la colaboración ATLAS del CERN desde 2016, soy coautor de 335 publicaciones que pueden consultarse en inspireHEP: http://inspirehep. net/search?p=joaquin+hoya\&action_search=Buscar\&sf=earliestdate. En base a mis trabajos, reflejados en parte en las notas internas listadas más abajo, destaco las siguientes publicaciones por su relevancia.

1. "Search for new phenomena in final states with large jet multiplicities and missing transverse momentum using $\sqrt{s}=13 \mathrm{TeV}$ protonproton collisions recorded by ATLAS in Run 2 of the LHC" ATLAS Collaboration. http://cds.cern.ch/record/2710420. Feb, 2020. ATLASCONF-2020-002.

2. "Performance of electron and photon triggers in ATLAS during LHC Run 2"

ATLAS Collaboration. 10.1140/epjc/s10052-019-7500-2. Eur. Phys. J. C (2020)

3. "Search for photonic signatures of gauge-mediated supersymmetry in $13 \mathrm{TeV}$ pp collisions with the ATLAS detector"

ATLAS Collaboration. doi.org/10.1103/PhysRevD.97.092006. Phys. Rev. D 97 (2018) 092006.

4. "ATLAS Electron and Photon Trigger"

J. Hoya, on behalf of the ATLAS Collaboration. doi.org/10.22323/1.321.0027. PoS(LHCP2018)027. 2018.

5. "Performance of the ATLAS Trigger System in 2015"

ATLAS Collaboration. doi.org/10.1140/epjc/s10052-017-4852-3. Eur. Phys. J. C77 (2017) 317.

6. "Search for supersymmetry in a final state containing two photons and missing transverse momentum in $\sqrt{s}=13 \mathrm{TeV}$ pp collisions at the LHC using the ATLAS detector"

ATLAS Collaboration. doi.org/10.1140/epjc/s10052-016-4344-x. Eur. Phys. J. C 76 (2016) 517. 
Publicaciones en etapa de aprobación interna

El siguiente trabajo está pendiente de la aprobación final interna de la colaboración ATLAS para hacerlo público.

1. "Search for SUSY signatures in events with photons, missing transverse energy and jets using $139 \mathrm{fb}^{-} 1^{\prime \prime}$

The ATLAS Collaboration.

Notas internas de ATLAS

1. "Performance of ATLAS Electron and Photon triggers during LHC Run 2"

T. Bold, K. Burka, D. Damazio, M. de Araujo, W. Freund, I. Grabowska-Bold, J. Hoya, T. Hryn'ova, P. Janus, S. Jones, D. Koeck, J. Kremer, D. Millar, F. Monticelli, G. Orellana, J. Pinto, B. Sefarzadeh, J. Seixas, M. Spina, H. Wahlberg, R. White. ATL-COM-DAQ-2019-032. CERN, 2019.

2. "Search for SUSY signatures in events with photons, missing transverse energy and jets using $140 \mathrm{fb}^{-} 1^{\prime \prime}$

F. Alonso, F. Arduh, M.T. Dova, J. Hoya, G. Orellana, H. Wahlberg. ATLCOM-PHYS-2018-1420. CERN, 2018.

3. "The Quest for Supersymmetry in Final States with many Hadronic Jets, using the Full Run 2 ATLAS Data"

A. Barr, C. Gwenlan, A. Sfyrla, K. Nagai, TJ. Khoo, M. Nelson, M. Valente, A. O'neill, J. Hoya, H. Wahlberg. ATL-COM-PHYS-2018-1419. CERN, 2018.

4. "Search for direct pair production of a chargino and a neutralino decaying via a lepton plus photons from the $125 \mathrm{GeV}$ Higgs boson: update with full Run-II data sample"

F. Alonso, M. Gignac, J. Hoya, O. Jinnouchi, B. Laforge, A. Leopold, A. Litke, S. Mazza, B. Schumm, H. Wahlberg. ATL-COM-PHYS-2018-753. CERN, 2018 .

5. "ATLAS Electron and Photon Trigger"

J. Hoya. ATL-COM-DAQ-2018-126. CERN, 2018.

6. "Search for supersymmetry in events with photons, jets and missing transverse momentum with $36 \mathrm{fb}^{-1}$ of data at $13 \mathrm{TeV}$ "

F. Alonso, F. Arduh, J. Hoya, M.T. Dova, H. Wahlberg, G. Orellana. ATLCOM-PHYS-2016-1662. CERN, 2016.

Notas cortas de ATLAS

1. "Performance of Photon triggers in ATLAS during LHC Run-2" F. Monticelli, G. Orellana, J. Hoya, H. Wahlberg. ATL-COM-DAQ-2018-184. CERN, 2018.

2. "Electron and photon trigger efficiencies and rates using 2017 data for LHCC"

A. Ruiz-Martinez, F. Monticelli, S. Jones, J. Hoya. ATL-COM-DAQ-2017-117. CERN, 2017. 
3. "Electron and photon trigger efficiency plots using the full 2016 dataset"

A. Ruiz-Martinez, F. Monticelli, J. Hoya, S. Jones. ATL-COM-DAQ-2017-015. CERN, 2017.

4. "Electron/photon trigger efficiency plots for ICHEP2016"

M. Backes, A. Ruiz-Martinez, S. Jones, F. Monticelli, J. Hoya. ATL-COMDAQ-2016-086. CERN, 2016.

5. "Photon trigger performance in 2015 ATLAS data"

G. Pasztor, R. White, F. Monticelli, J. Hoya, H. Wahlberg. ATL-COM-DAQ2015-101. CERN, 2015. 


\section{Índice general}

$\begin{array}{lll}\text { Publicaciones científicas } & \text { IV }\end{array}$

Introducción 3

1. Supersimetría: SUSY 4

1.1. Simetría . . . . . . . . . . . . . . . . . 4

1.2. El Modelo Estándar de las partículas . . . . . . . . . . . . 5

1.2.1. Interacciones electrodébiles . . . . . . . . . . . . . 7

1.2.2. Ruptura espontánea de simetría . . . . . . . . . . . . . 8

1.2.3. Interacciones fuertes . . . . . . . . . . . . . . 10

1.2.4. Interacciones hadrónicas en un colisionador protón-protón . . 12

1.2.5. Limitaciones del Modelo Estándar . . . . . . . . . . . . . . 15

1.3. Simetría Fermión-Bosón . . . . . . . . . . . . . . . . . 17

1.3.1. El álgebra de SUSY . . . . . . . . . . . . . . . . . . . . . . . 17

1.4. El Modelo Estándar Supersimétrico Mínimo: MSSM . . . . . . . . . . 18

1.4.1. Rompimiento de Supersimetría . . . . . . . . . . . . . 18

1.4.2. Parámetros libres de la teoría . . . . . . . . . . . . . . . . . 20

1.4.3. Paridad R . . . . . . . . . . . . . . . . . 21

1.4.4. El espectro de masa del MSSM . . . . . . . . . . . . . 21

1.5. Modelos para el mecanismo de rompimiento de SUSY . . . . . . . . . 23

1.5.1. Propuestas para las interacciones mediadoras . . . . . . . . . 23

1.5.2. Stealth SUSY . . . . . . . . . . . . . . . . 25

2. El experimento ATLAS 27

2.1. El Gran Colisionador de Hadrones . . . . . . . . . . . . . . . . . . 27

2.2. El detector ATLAS . . . . . . . . . . . . . . . . . . . . . . . . . . . . . . . . . . . . . . . .

2.2.1. Sistema de coordenadas . . . . . . . . . . . . . 31

2.2.2. Sistema de imanes . . . . . . . . . . . . . . . 34

2.2.3. Detector interno . . . . . . . . . . . . . . . . . . . . . . . . . 34

2.2.4. Calorímetros . . . . . . . . . . . . . 37

2.2.5. Espectrómetro de Muones . . . . . . . . . . . . . . 39

2.3. El sistema de trigger . . . . . . . . . . . . . . . . . . . 39

2.3.1. L1: Trigger basado en Hardware . . . . . . . . . . . . . . . . 40

2.3.2. HLT: Trigger basado en Software . . . . . . . . . . . . . . . 41

2.3.3. Cadenas y menú del trigger . . . . . . . . . . . . . . . 42

2.4. Manejo y distribución de datos y recursos computacionales . . . . . . 42

2.5. Toma de datos durante el Run-2 . . . . . . . . . . . . . . . . . . . . . 44

2.6. Simulaciones Monte Carlo . . . . . . . . . . . . . . . . . . . . 46 
3. Objetos físicos en ATLAS 47

3.1. Fotones y Electrones . . . . . . . . . . . . . . . . . . 47

3.1.1. Reconstrucción . . . . . . . . . . . . . . . . 47

3.1.2. Identificación . . . . . . . . . . . . . . . . . . . . . 48

3.1.3. Aislamiento . . . . . . . . . . . . . . . . 51

3.2. Muones ............................. 52

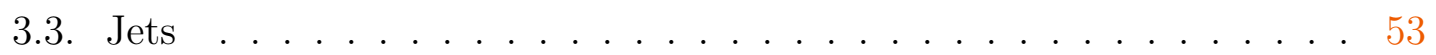

3.3.1. Jets provenientes de quarks b: b-jets . . . . . . . . . 55

3.4. Momento transverso faltante . . . . . . . . . . . . . . . . 56

4. Eficiencia del trigger de fotones y multijets 58

4.1. El método Bootstrap . . . . . . . . . . . . . . . . . . . 58

4.2. Eficiencia del trigger de fotones . . . . . . . . . . . . . 60

4.2.1. Resultados de las eficiencias del trigger de fotones . . . . . . . 62

4.3. Método de estimación de fondo para cálculos de eficiencias . . . . . . 62

4.3.1. Resultados de las eficiencias con sustracción de fondo para

datos de $2015 \ldots \ldots$. . . . . . . . . . . . . . . . 67

4.4. Eficiencia del trigger de multijets . . . . . . . . . . . . . 69

4.4.1. Comparación de las eficiencias del trigger de múltiples jets

versus EMTopo y PFlow jets . . . . . . . . . . . . . . 69

4.5. Propuesta de nuevo trigger combinado de multijets y un fotón . . . . 70

5. Estrategia de búsqueda de SUSY en estados finales con fotones + $\begin{array}{ll}\text { jets }+E_{\mathrm{T}}^{\text {miss }} & 76\end{array}$

5.1. Muestra de datos y selección de eventos . . . . . . . . . . . . . . 77

5.1.1. Fotones . . . . . . . . . . . . . . . 77

5.1.2. Electrones . . . . . . . . . . . . . . . . . 78

5.1.3. Muones ............................ 79

5.1.4. Jets . . . . . . . . . . . . . . . . . 79

5.1.5. Eliminación de solapamiento . . . . . . . . . . . . . . . . 79

5.1.6. Momento transverso faltante . . . . . . . . . . . . . . . 80

5.2. Muestras MC de señal y de fondos del SM . . . . . . . . . . . . . 80

5.2.1. Muestras de señal . . . . . . . . . . . . . . . . 80

5.2.2. Muestras de fondo . . . . . . . . . . . . . . . . . 83

5.3. Regiones de señal, control y validación . . . . . . . . . . . 86

5.3.1. Optimización y definición de las regiones de señal . . . . . . . 87

5.3.2. Regiones de control y validación . . . . . . . . . . . . . . 93

5.4. Estimación del fondo en base a técnicas de datos . . . . . . . . . . . 100

5.4.1. Jets falseando fotones . . . . . . . . . . . . . . . 100

5.4.2. Electrones falseando fotones . . . . . . . . . . . . 103

5.5. Tratamiento estadístico e interpretación de los resultados . . . . . . . 106

5.5.1. Test de hipótesis . . . . . . . . . . . . . . . . . 107

5.5.2. Construcción del modelo . . . . . . . . . . . . . . . . . 109

5.5.3. Flujo de una búsqueda . . . . . . . . . . . . . . . . . 110

6. Resultados e interpretación de la búsqueda de SUSY en estados

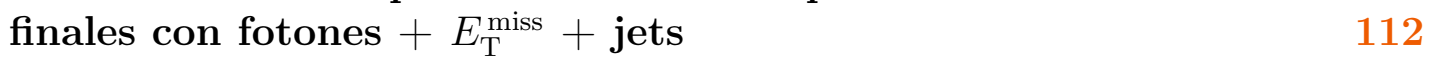

6.1. Incertezas sistemáticas . . . . . . . . . . . . . . . . . . . . . 112

6.1.1. Incertezas experimentales . . . . . . . . . . . . 112 
6.1.2. Incertezas originadas en métodos data-driven . . . . . . . . 114

6.1.3. Incertezas teóricas . . . . . . . . . . . . . . . . . 115

6.2. Ajuste de solo-fondo para todo Run-2 . . . . . . . . . . . . . . . 115

6.2.1. Resultados en las regiones de control . . . . . . . . . . . . 115

6.2.2. Resultados en las regiones de validación . . . . . . . . . 116

6.2.3. Resultados en las regiones de señal . . . . . . . . . . . . . 118

6.3. Resultados en las regiones de señal para datos de 2015 y 2016 . . . . 136

6.4. Límites independientes del modelo . . . . . . . . . . . . . . . . . . 137

6.5. Límites dependientes del modelo . . . . . . . . . . . . . . . . . . 139

7. Nuevas búsquedas en modelos extendidos 141

7.1. Modelos y simulaciones . . . . . . . . . . . . . . . . . . . . . 142

7.2. Selección de objetos y distribuciones . . . . . . . . . . . . . . . . 143

7.3. Estudios preliminares de factibilidad . . . . . . . . . . . . . . 145

7.3.1. Impacto de selección de eventos por un trigger combinado . . 146

7.3.2. Reinterpretación simple de Nueva Física con Stealth SUSY . . 148

8. Conclusión

Bibliografía 


\section{Introducción}

La física de partículas tiene como objetivos descubrir las leyes que rigen nuestro universo y revelar los componentes elementales a partir de los cuales se construye. Desde principios del siglo XX, la estrecha interacción entre la física de partículas teórica y experimental ha sido de enorme importancia para el desarrollo del campo. Juntos, los conceptos teóricos y las observaciones experimentales dieron lugar a lo que se conoce como el Modelo Estándar (SM) que sintetiza nuestra comprensión actual de las partículas fundamentales y sus interacciones. El SM proporciona un marco notablemente preciso de los fenómenos actualmente entendidos de nuestro universo, encapsulando su complejidad en solo dos tipos de partículas: fermiones y bosones. Aunque es considerado un moderno triunfo de la física, el SM no explica todos los procesos observados en la naturaleza: describe solo una fracción del contenido de materia del universo y no proporciona candidatos viables a partículas para la materia oscura que representan una proporción significativa del universo, y, aún más intrigante, por qué existe una asimetría entre la materia y la antimateria, entre otros interrogantes abiertos. Desde hace varios años, los físicos se han abocado a la investigación de la física más allá del SM, buscando proporcionar extensiones al exitoso modelo actual. El laboratorio CERN (Organización Europea para la Investigación Nuclear) tiene un extenso programa de investigación para la búsqueda de nueva física, con experimentos basados en el Gran Colisionador de Hadrones (LHC), en particular el detector ATLAS en cuyo marco se realizó la presente tesis. En diciembre de 2018 concluyó el segundo período de toma de datos del LHC, llamado Run-2. Desde entonces el LHC cesó las actividades para iniciar las actualizaciones y mejoras planificadas para retomar en 2021 con colisiones entre protones a mayor energía de centro de masa y mayor luminosidad.

En esta tesis se describe una búsqueda de nueva física con fotones aislados de alto $p_{\mathrm{T}}$, jets y alto momento transversal faltante inspirada por modelos de Supersimetría, que es una de las teorías mejor motivadas teóricamente para la física más allá del Modelo Estándar. La búsqueda se realiza con el conjunto de datos de colisiones pp a $\sqrt{s}=13 \mathrm{TeV}$ registrado entre 2015 y 2018 con el detector ATLAS en el LHC, lo que corresponde a una luminosidad total integrada de $139 \mathrm{fb}^{-1}$.

Análisis previos se han desarrollado en ATLAS usando el conjunto de datos a energía de colisiones de $8 \mathrm{TeV}$ [1], y a $13 \mathrm{TeV}$ con los primeros datos colectados en 2015 y 2016 [2]. Estos estudios entre otros, no han mostrado evidencia de excesos de datos significativos sobre las predicciones del Modelo Estándar. Para la investigación desarrollada en la presente tesis, se incluyeron cuatro veces más de datos a energía de colisión de $13 \mathrm{TeV}$, se incluyeron mejoras y optimizaciones de los métodos de reconstrucción y selección de los objetos físicos involucrados en los procesos bajo estudio, se implementaron distintas técnicas para la determinación de los fondos del SM - sin dudas el desafío mayor en la búsqueda experimental de nueva física- y se 
diseñó una estrategia de búsqueda utilizando nuevas regiones del espacio observable donde la señal de física nueva podría manifestarse (regiones de señal), y nuevas regiones de control y validación para la determinación de los fondos contaminantes a la señal, todas optimizadas para aumentar la sensibilidad a descubrimiento o extender los límites de exclusión actuales sobre las masas de las partículas supersimétricas producidas (gluinos y neutralinos) hasta valores nunca antes alcanzados.

Las características experimentales del estado final buscado están motivadas por modelos de supersimetría (SUSY) [3, 4, 5, 6, 7, 8, 9], con ruptura de simetría con mediadores de gauge (GMSB) [10, 11, 12]. Éstas suponen un sector oculto en el que la supersimetría se rompe, y la ruptura de la simetría se comunica al sector visible a través de las interacciones de bosones de gauge del SM.

La partícula supersimétrica más liviana (LSP) en el GMSB es el gravitino ultraliviano $(\widetilde{G})$ que, bajo ciertas circunstancias, es un candidato viable para la materia oscura $[13,14]$. La fenomenología de los modelos GMSB está determinada por la naturaleza de la siguiente partícula supersimétrica a la más liviana (NLSP) que, para gran parte del espacio de parámetros GMSB, es el neutralino $\widetilde{\chi}_{1}^{0}$ más liviano. Los neutralinos son mezclas de autoestados de gauginos $\left(\tilde{B}, \tilde{W}^{0}\right)$ y higgsinos $\left(\tilde{H}_{u}^{0}, \tilde{H}_{d}^{0}\right)$ y, por lo tanto, el neutralino más liviano se descompone en un $\widetilde{G}$ y un $\gamma, Z$, o $h$ (el bosón neutral más liviano del sector de Higgs supersimétrico, que se supone compatible con el bosón de Higgs observado en ATLAS y CMS [15, 16]).

Entre los años 2015 y 2018 (Run-2), el LHC funcionó a una energía del centro de masa de $13 \mathrm{TeV}$ y una luminosidad que excedió los $10^{34} \mathrm{~cm}^{-2} \mathrm{~s}^{-1}$. A mayor luminosidad, el número y complejidad de los requerimientos del trigger (sistema de selección en-línea) aumenta para satisfacer los objetivos físicos, manteniendo al mismo tiempo las tasas de salida en el orden de los $100 \mathrm{kHz}$. Para el programa de física de ATLAS es de máxima relevancia el rendimiento eficiente del sistema de trigger para seleccionar eventos de interés, con un alto rechazo de aquellos procesos de QCD conocidos que constituyen los fondos dominantes en el LHC. Las partículas que interactúan a través de las interacciones electrodébiles, como los electrones y los fotones, están presentes en muchos procesos de interés del SM así como en la búsqueda de física más allá del SM, como es el caso de la búsqueda de SUSY desarrollada en capítulos siguientes de esta tesis. La capacidad de seleccionar, en particular los fotones, de manera efectiva es un ingrediente crucial para el experimento ATLAS y, debido al rol central que juegan los fotones en la búsqueda que se realiza en esta tesis, parte del trabajo se ha centrado en la evaluación de la performance del detector para seleccionar eventos que contengan dichos objetos físicos.

En particular, se presenta la evolución de los triggers de fotones para la toma de datos de ATLAS de colisiones $p p$ en 2015-2018 y se computan sus eficiencias. Se estudia además, la performance para los triggers de jets necesarios para un análisis específico con multijets en el estado final.

La tesis está organizada de la siguiente manera. Primero se introduce SUSY, como una teoría atractiva para extender el SM y que, a su vez, brinda modelos con una rica fenomenología comprendiendo una gran variedad de estados finales en los eventos colectados por el detector ATLAS del LHC. El acelerador LHC y el detector ATLAS se describen en el Capítulo 2, donde se detalla también el sistema de trigger. El siguiente capítulo discute la reconstrucción e identificación de los objetos físicos utilizados en el presente trabajo. En el Capítulo 4 se presenta la técnica utilizada para la evaluación de la eficiencia del trigger de fotones y de jets, y los resultados 
obtenidos para el conjunto de datos colectados entre 2015 y 2018. Luego se desarrolla la estrategia seguida para la búsqueda de SUSY en estados finales con fotones, jets y momento transverso faltante. Aquí se detallan la selección de objetos utilizados, la optimización de las regiones de señal y definiciones de las regiones de control y validación, las técnicas utilizadas para estimar los fondos y el método estadístico utilizado. En el Capítulo 6 se muestran las incertezas sistemáticas y los resultados obtenidos en el contexto de la búsqueda de nueva física bajo estudio. Especulando con nuevos escenarios, el Capítulo 7 discute la factibilidad de una búsqueda en el marco de un modelo de Supersimetría más complejo y, por consiguiente con mayor número de parámetros libres, con un nuevo sector donde la ruptura de Supersimetría está prácticamente ausente, Stealth SUSY. En el Capítulo 8 se resumen y discuten en contexto, los resultados de esta tesis a modo de conclusión. 


\section{Capítulo 1}

\section{Supersimetría: SUSY}

En este capítulo se detalla el marco teórico en el que se basa la búsqueda experimental desarrollada en esta tesis. Debido a que la idea central es buscar evidencia de nueva física o física más allá del SM, iniciamos este capítulo con un breve comentario sobre un concepto central en física: la simetría. Luego se hace una breve descripción del SM comentando sus virtudes y limitaciones. A continuación, se describe en términos generales la teoría elegida para ir más allá del SM, Supersimetría (SUSY), mostrando cómo puede solucionar algunas de las patologías del SM y al mismo tiempo brindar, de manera colateral, posibles respuestas a otras incógnitas actuales que el SM no puede dar respuesta (viable candidato a materia oscura por ejemplo). Se describe entonces la mínima extensión supersimétrica del SM (MSSM) de modo general. Finalmente, se discute sobre el rompimiento de SUSY, y se describen los modelos particulares de mediación de rotura de supersimetría por interacciones de gauge (GMSB) y un caso particular de espectro supersimétrico degenerado (Stealth SUSY), con los que se trabaja.

\subsection{Simetría}

El concepto de simetría está presente en incontables aspectos de nuestra vida cotidiana, y es en física donde juega un papel central a la hora de formular las teorías que describen la naturaleza. Un ejemplo intuitivo de este concepto son las baldosas de Penrose o Penrose Tiling, que se muestra en la Figura 1.1, y tiene la característica de no presentar simetría traslacional, pero es simétrico bajo reflexión y bajo rotaciones discretas de orden 5 (es decir de $72^{\circ}$ ). La definición de simetría provista por Hermann Weyl,

"A symmetry is some operation that one can do to a thing such that it still looks the same afterwards." H. Weyl

posee profundas implicancias como demostró Emily Noether, en el famoso teorema que lleva su nombre, donde cada simetría continua está conectada con una cantidad conservada (una corriente continua con carga conservada). Este teorema relaciona simetrías observadas y leyes de conservación con la estructura de la teoría. Vale resaltar entonces este trabajo fundacional de Noether, que sentó las bases para la formulación y comprensión de las teorías física que la sucedieron y siguen hasta la actualidad. Resulta ser una guía en la construcción de muchos modelos de física BSM. 


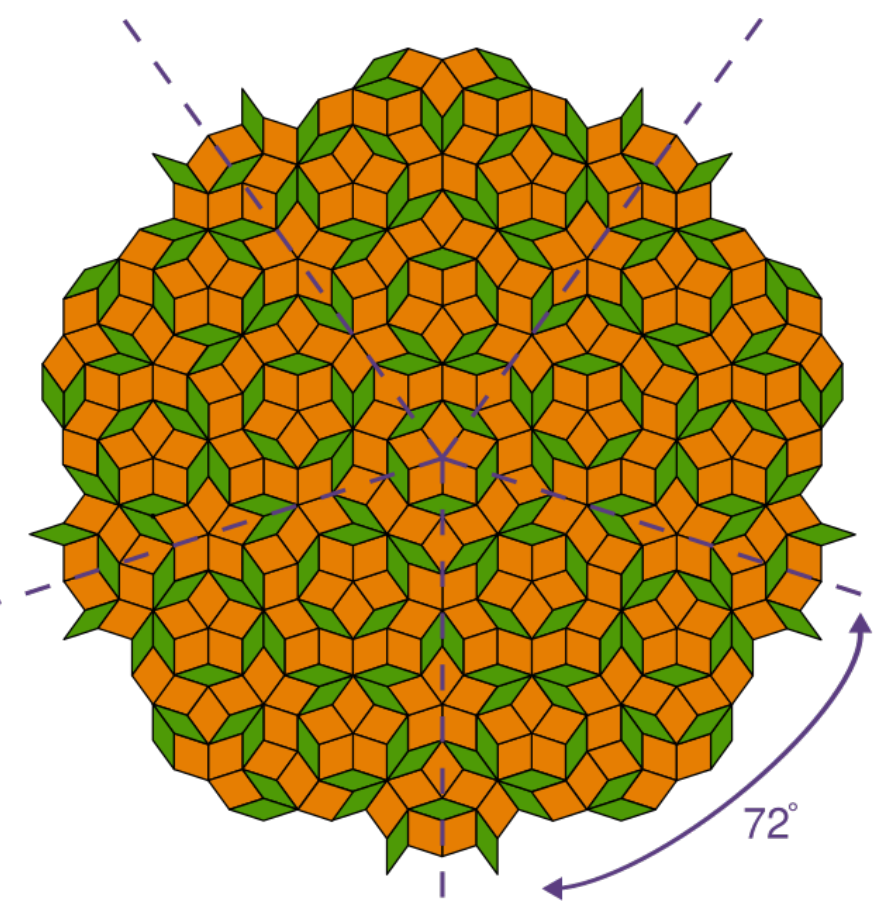

Figura 1.1: Ejemplo de simetría: Penrose Tiling.

Las simetrías en física se pueden dividir en discretas y continuas. Como ejemplos de las primeras se pueden mencionar Paridad (P), Conjugación de carga (C) o Inversión temporal (T). Las continuas, a su vez, se pueden dividir en geométricas e internas, donde las geométricas son aquellas que se aplican sobre las coordenadas espacio temporales y muestran situaciones observacionales, como por ejemplo rotaciones o traslaciones. Por su parte las internas, que se aplican sobre el espacio interno de los campos que describen la teoría, pueden ser globales o locales (cuyos parámetros están conectados con el espacio-tiempo). Cada simetría tiene su grupo asociado, con lo que la teoría de grupos es el lenguaje natural para tratarlas.

Por último, antes de detallar el Modelo Estándar de las partículas, es interesante resaltar que se busca la simetría en las ecuaciones de movimiento dado que la física solo depende de ellas. En el caso de la teoría cuántica de campos, existen casos en los cuales la ecuación de movimiento es simétrica y el estado fundamental no respeta la simetría. Se dice entonces que la simetría se rompe de manera espontánea a bajas energías, y la dinámica permanece constreñida por la simetría. Se vuelve más adelante sobre esta idea al discutir el mecanismo de Higgs.

\section{2. $\quad$ El Modelo Estándar de las partículas}

El Modelo Estándar es una teoría cuántica de campos renormalizable que provee una descripción precisa y sin precedentes, de los campos de las partículas elementales conocidas y de las interacciones fuerte, débil y electromagnética. La interacción gravitatoria, la cuarta conocida en la naturaleza, no se incluye en esta descripción. Aquellas interacciones surgen de pedir que la teoría sea invariante bajo transformaciones de gauge locales del grupo de simetría: $S U(3)_{C} \times S U(2)_{L} \times U(1)_{Y}$, y se detalla brevemente a continuación siguiendo en gran medida a [17], [18] y [19]. Se tiene que: 


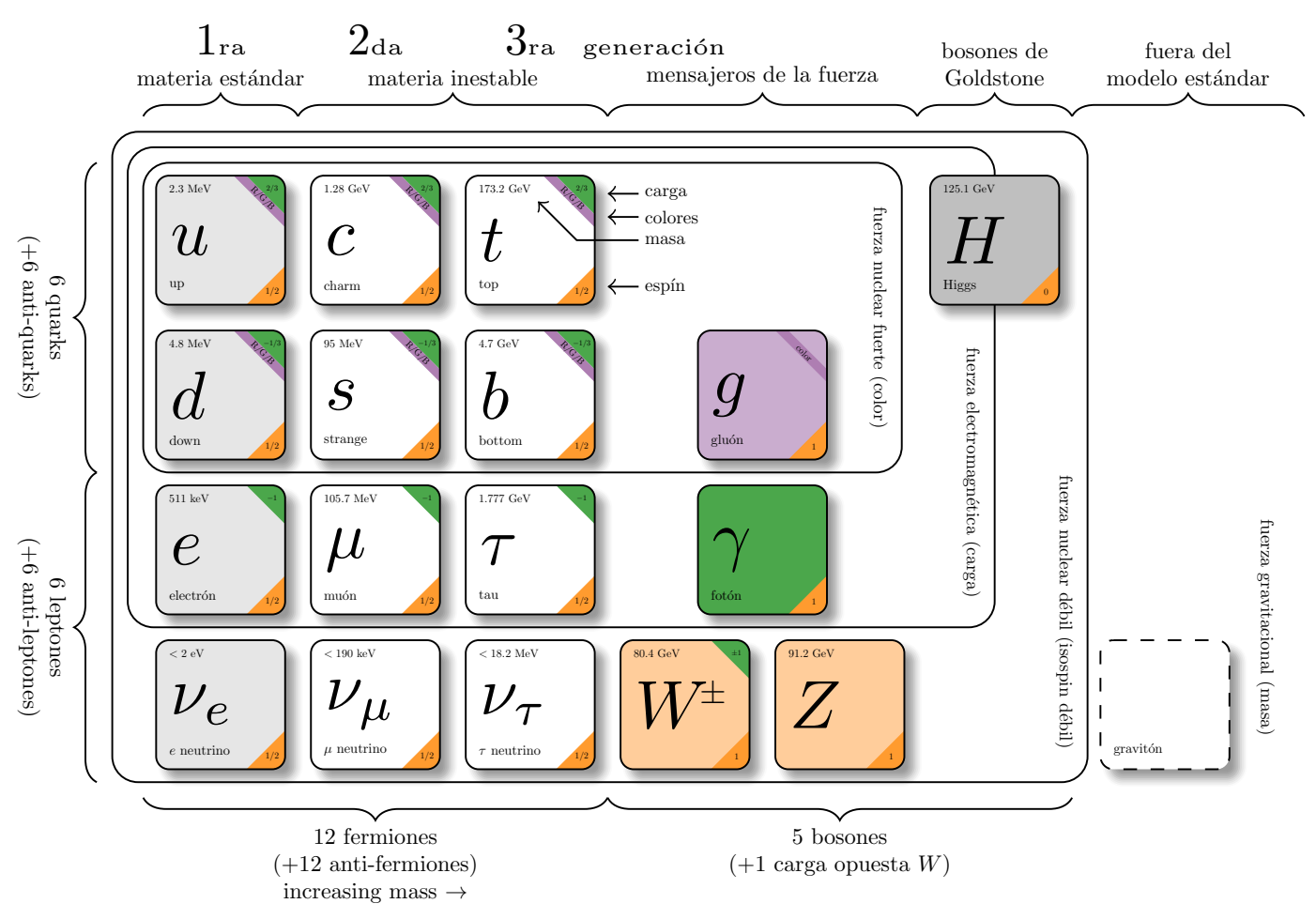

Figura 1.2: Resumen de las partículas y las interacciones del Modelo Estándar. Gráfico basado en [20] y el trabajo de Carsten Burgard.

- El grupo $S U(2)_{L} \times U(1)_{Y}$ describe el sector electrodébil de Isospin débil e Hipercarga.

- El grupo $S U(3)_{C}$ describe la cromodinámica cuántica (QCD).

En las secciones siguientes se detallan estas interacciones, pero antes se presenta una descripción general de las partículas que componen el Modelo Estándar, resumida en la Figura 1.2.

Dichas partículas pueden dividirse en primer lugar entre partículas interactuantes, que son fermiones (espín semientero) y partículas mediadoras de las interacciones, que son bosones (espín entero). Es decir que se tienen campos fermiónicos de materia interactuando a través de campos de gauge bosónicos. Los fermiones del SM son 12 (más sus anti partículas) que se dividen a su vez en leptones y quarks, y en tres familias o generaciones (de cuatro partículas) de diferentes sabor y crecientes masas. En cada familia hay dos leptones, uno cargado (carga eléctrica $Q=-1 e$ ) y otro neutro (el neutrino) al que se considera no masivo en este modelo (siendo que distintos experimentos le han puesto cotas a sus masas, véase Sección 1.2.5). De los dos quarks, la componente up tiene carga eléctrica $Q=\frac{2}{3} e$ con isospin $I=+\frac{1}{2}$, mientras la componente down tiene carga eléctrica $Q=-\frac{1}{3} e$ con isospin $I=-\frac{1}{2}$. El fotón es el mediador de las interacciones electromagnéticas, es no masivo y no posee carga eléctrica. Por su parte, los bosones $W \pm$ (con carga eléctrica $Q= \pm 1 e$ ) y $Z$ (neutro eléctricamente), mediadores de la fuerza débil, poseen masas de $80.4 \mathrm{GeV}$ y $91.2 \mathrm{GeV}$, respectivamente. Finalmente, las interacciones fuertes son mediadas por los ocho gluones del modelo (uno por cada generador de $S U(3)_{C}$ ) que sólo poseen 
carga de color y son no masivos. El último bosón del modelo es el bosón de Higgs que, a diferencia de los bosones vectoriales anteriores, es un escalar fundamental. Se vuelve sobre éste en las sucesivas secciones donde se resalta su papel central en la generación de masas para fermiones y bosones a través del proceso de ruptura espontánea de la simetría electrodébil.

\subsubsection{Interacciones electrodébiles}

Las interacciones electrodébiles satisfacen la simetría de gauge del grupo $S U(2)_{L} \times$ $U(1)_{Y}$. El grupo $S U(2)_{L}$, llamado isospin débil, actúa sólo sobre los fermiones de quiralidad izquierda, y el grupo $U(1)_{Y}$ se llama de hipercarga y actúa sobre ambas quiralidades de manera vectorial. El grupo $S U(2)_{L} \times U(1)_{Y}$ tiene cuatro generadores, de los cuales tres pertenecen al isospin débil: $T_{i}=\frac{\sigma_{i}}{2}$, con $i=1,2,3$ y $\sigma_{i}$ las matrices de Pauli, y uno al grupo de hipercarga: $\frac{Y}{2}$. Los fermiones izquierdos transforman como dobletes bajo $S U(2)_{L}, f_{L} \rightarrow e^{i \vec{T} \vec{\theta}} f_{L}$ con

$$
f_{L}=\left(\begin{array}{c}
\nu_{L} \\
e_{L}
\end{array}\right),\left(\begin{array}{c}
u_{L} \\
d_{L}
\end{array}\right), \cdots
$$

mientras que los fermiones derechos lo hacen como singletes $f_{R} \rightarrow f_{R}$ con

$$
f_{R}=e_{R}, u_{R}, d_{R}, \cdots
$$

Esta distinción entre izquierda y derecha implica que el modelo $S U(2)_{L} \times U(1)_{Y}$ es una teoría quiral.

La carga eléctrica está relacionada con la tercer componente del isospin débil $T_{3}$ y la hipercarga $Y$, de acuerdo a la fórmula de Gell-Mann Nishijima:

$$
Q=T_{3}+\frac{Y}{2}
$$

En cada generación de quarks y leptones, los números cuánticos se repiten.

El número de bosones de gauge asociados coincide con el número de generadores del grupo de simetría. Para el isospin débil se tienen 3 bosones de $S U(2)_{L}$ : $W_{\mu}^{1}, W_{\mu}^{2}, W_{\mu}^{3}$, y para $U(1)_{Y}$ se tiene un bosón de hipercarga: $B_{\mu}$.

La simetría global $S U(2)_{L} \times U(1)_{Y}$ pasa a ser local, reemplazando en el lagrangiano la derivada de los campos por la derivada covariante:

$$
D_{\mu}=\partial_{\mu}-i g \vec{T} \cdot \vec{W}_{\mu}-i g^{\prime} \frac{Y}{2} B_{\mu}
$$

donde $\mathrm{g}$ es la constante de acoplamiento de $S U(2)_{L}$ y g' de $U(1)_{Y}$.

Se definen los tensores de campo de Yang-Mills $\vec{F}_{\mu \nu}$ para $S U(2)_{L}$ y $B_{\mu \nu}$ para $U(1)_{Y}$ como:

$$
\begin{gathered}
\vec{F}_{\mu \nu}=\partial_{\mu} \vec{W}_{\nu}-\partial_{\nu} \vec{W}_{\mu}+g \vec{W}_{\mu} \times \vec{W}_{\nu} \\
B_{\mu \nu}=\partial_{\mu} B_{\nu}-\partial_{\nu} B_{\mu}
\end{gathered}
$$


La densidad lagrangiana electrodébil puede escribirse entonces como la suma del lagrangiano fermiónico con las interacciones de gauge y los términos cinéticos para los campos de gauge introducidos:

$$
\mathcal{L}_{E W}=-\frac{1}{4} \vec{F}_{\mu \nu} \cdot \vec{F}^{\mu \nu}-\frac{1}{4} B_{\mu \nu} B^{\mu \nu}+\sum_{f=l, q} \bar{f} i \gamma^{\mu} D_{\mu} f
$$

Hasta el momento no se han incluido términos de masa para los fermiones o los bosones de gauge, ya que los típicos términos de masa de Dirac, rompen la simetría, como por ejemplo con un lagrangiano de la forma:

$$
\mathcal{L}=-m \bar{\psi} \psi=-m\left(\bar{\psi}_{L} \psi_{R}+\bar{\psi}_{R} \psi_{L}\right)
$$

que es inaceptable con $\psi_{L}$ un doblete y $\psi_{R}$ un singlete de $S U(2)_{L}$.

Por lo tanto, y debido a que las masas de dichas partículas se conocen de manera experimental (y sus valores se muestran en la Figura 1.2), se necesita introducir un mecanismo extra que las genere. Se agrega entonces un campo escalar, y se considera la simetría como una simetría latente, presente en el lagrangiano pero no respetada por el valor de expectación del vacío (vev) del campo. Los estados de vacío degenerados respetan la simetría $S U(2)_{L} \times U(1)_{Y}$, y es la elección particular de uno de ellos lo que genera el rompimiento $S U(2)_{L} \times U(1)_{Y} \rightarrow U(1)_{E M}$. Este mecanismo (al que un tanto injustamente se lo suele referir en la literatura como mecanismo de Higgs) en el que se produce un rompimiento espontáneo de la simetría se describe brevemente a continuación y se debe al trabajo de Anderson[21], Nambu[22], Higgs[23], Englert, Brout[24], Guralnik, Hagen y Kibble [25, 26].

\subsubsection{Ruptura espontánea de simetría}

El mecanismo de Higgs antes mencionado, implica el rompimiento espontáneo de la simetría electrodébil $S U(2)_{L} \times U(1)_{Y}$ en $U(1)_{E M}$, y provee las masas de los fermiones y los bosones débiles $W^{ \pm}$y $Z$. Para ello, se introduce un campo escalar complejo, llamado campo de Higgs, que agrega un nuevo bosón en la teoría que es un doblete de isospin y que se acopla con los campos $\vec{W}_{\mu}$ y $B_{\mu}$ :

$$
\Phi=\left(\begin{array}{c}
\phi_{+} \\
\phi_{0}
\end{array}\right)=\frac{1}{\sqrt{2}}\left(\begin{array}{c}
\phi_{1}+i \phi_{2} \\
\phi_{3}+i \phi_{4}
\end{array}\right)
$$

donde $\phi_{+} \mathrm{y} \phi_{0}$ son complejos, con carga eléctrica igual a sus subíndices y $Y_{\Phi}=+1$.

Además del comportamiento cinemático de este campo, que tendrá la derivada covariante (Ec. 1.1), se agrega en el lagrangiano una interacción $V\left(\Phi^{\dagger} \Phi\right)$, de manera que respete la simetría $S U(2)_{L} \times U(1)_{Y}$, pero que rompa la simetría del vacío, es decir, que el mínimo del potencial esté infinitamente degenerado. Por lo tanto, el lagrangiano tendrá la forma:

$$
\mathcal{L}_{\text {Higgs }}=\left(D_{\mu} \Phi\right)^{\dagger}\left(D^{\mu} \Phi\right)^{\dagger}-V\left(\Phi^{\dagger} \Phi\right)
$$

El potencial se elige para que cumpla los requerimientos antes mencionado, como: 


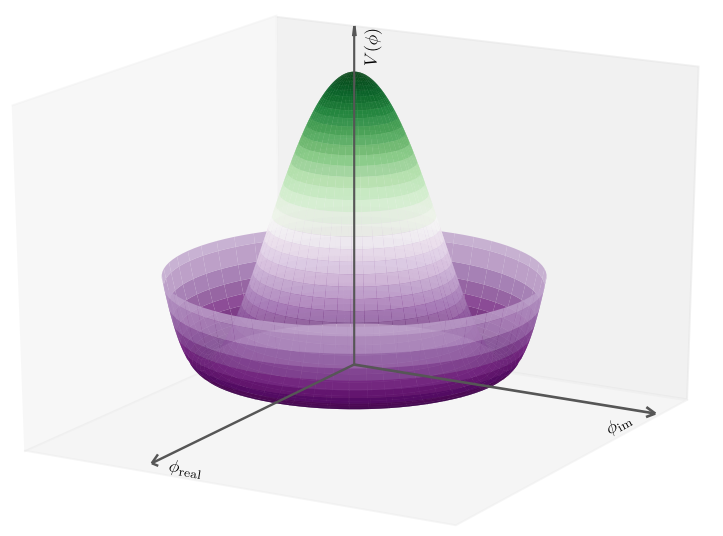

Figura 1.3: Potencial de Higgs con $\mu>0$.

$$
V\left(\Phi^{\dagger} \Phi\right)=-\mu^{2} \Phi^{\dagger} \Phi+\lambda\left(\Phi^{\dagger} \Phi\right)^{2}
$$

que depende de los parámetros $\mu$ y $\lambda$, y es invariante bajo la transformación de gauge local: $\phi \rightarrow \phi^{\prime}=e^{i \vec{\alpha} \cdot \vec{\sigma} / 2} \phi$, siendo $\sigma_{i}$ las matrices de Pauli y $\alpha_{i}$ parámetros. En este potencial, se requiere $\lambda>0$ para tener una cota inferior en el potencial, y el comportamiento queda determinado entonces por el signo de $\mu$. Si $\mu<0, V(|\phi|)$ tiene un sólo mínimo en cero, y no se obtiene el comportamiento buscado. Sin embargo, si $\mu>0$, el potencial tiene la forma de sombrero mexicano que se muestra en la Figura 1.3, con un estado fundamental infinitamente degenerado.

El valor absoluto del campo en el mínimo, el vev, se encuentra que es $|\Phi|=\frac{v}{\sqrt{2}}$. De los infinitos posibles valores en que se puede satisfacer esta condición, se suele usar:

$$
\langle\Phi\rangle=\frac{1}{\sqrt{2}}\left(\begin{array}{l}
0 \\
v
\end{array}\right)
$$

con $\phi_{1}=\phi_{2}=\phi_{4}=0, \phi_{3}=v$, por lo que $v=\frac{\mu}{\sqrt{\lambda}} \sim 246 \mathrm{GeV}$, valor que se obtiene de mediciones de la constante de Fermi de las interacciones débiles. Cualquier elección de entre todos los posibles valores romperá la simetría $S U(2)_{L} \times U(1)_{Y}$.

Se puede estudiar el espectro desarrollando alrededor del vacío, por lo que se puede elegir

$$
\phi=\frac{1}{\sqrt{2}}\left(\begin{array}{c}
0 \\
v+H(x)
\end{array}\right)
$$

donde $H(x)$ son excitaciones del estado fundamental alrededor del mínimo, pero en la dirección perpendicular al círculo con los infinitos mínimos. Debido a la invariancia de gauge del potencial para las excitaciones alrededor del círculo de los mínimos, y de acuerdo al teorema de Goldstone, se deberían tener en principio tres bosones escalares no masivos asociados a los grados de libertad no radiales del campo. Se tiene una realización à la Nambu-Goldstone de la simetría U(1) antes mencionada. La arbitrariedad del gauge permite que los bosones de Goldstone sean absorbidos 
por los bosones $W^{ \pm}$y $Z$ (proveen las polarizaciones longitudinales que adquieren los campos de gauge). El desarrollo en la dirección radial da la masa de la excitación $\mathrm{H}, \sqrt{2 \lambda} v$ que es la masa del bosón de Higgs, y acoples cúbicos y cuárticos de este bosón.

Por lo tanto, gracias a este mecanismo, los cuatro bosones de gauge de la teoría electrodébil poseen las masas correspondientes. En concreto, el fotón sigue no masivo, $m_{W}=\frac{g v}{2}, m_{Z}=\frac{g}{2} \sqrt{g^{2}+g^{\prime 2}}$ y el ángulo de Weinberg $\theta_{W}$ (ángulo de mezcla débil) puede escribirse en función de estas masas como $\operatorname{sen}^{2}\left(\theta_{W}\right)=1-\frac{m_{W}^{2}}{m_{Z}^{2}}$.

El mecanismo de Higgs también permite obtener las masas para los fermiones, incluyendo en el lagrangiano términos con acoplamientos tipo Yukawa de la forma:

$$
\mathcal{L}_{Y}=-y_{f} \bar{\psi}_{L} \Phi \psi_{R}+\text { h.c. }
$$

donde $y_{f}$ son las matrices que describen los acoplamientos de Yukawa con el doblete de Higgs y los fermiones, y este lagrangiano es invariante de gauge ya que $\bar{\psi}_{L} \Phi \psi_{R}$ son singletes de $S U(2)_{L}$.

Si se desarrolla nuevamente alrededor del mínimo, se obtienen las masas para los fermiones como $m_{f}=\frac{y_{f} v}{\sqrt{2}}$.

\subsubsection{Interacciones fuertes}

Las interacciones fuertes entre quarks y gluones son descriptas por la cromodinámica cuántica en el SM y satisfacen la simetría de gauge del grupo $S U(3)_{C}$. La $C=R, B, G$ corresponde al número cuántico color, necesario para distinguir los tres posibles estados de los quarks. Los mesones y bariones, hadrones formados por dos o tres quarks respectivamente, son singletes "blancos" (carga de color neutra) de $S U(3)$.

El grupo de simetría $S U(3)_{C}$ tiene ocho generadores $T^{a}=\frac{\lambda_{\alpha \beta}^{a}}{2}$, siendo $\alpha$ y $\beta$ índices de color, $\lambda_{\alpha \beta}^{a}$ las ocho matrices de Gell-Mann y $a=1,2, \cdots, 8$ de la representación adjunta de $S U(3)$. Por lo tanto, se tiene ocho bosones de gauge, que serán los gluones $G_{\mu}^{a}$.

La simetría local $S U(3)_{C}$ se obtiene reemplazando en el lagrangiano la derivada de los campos por la derivada covariante:

$$
D_{\mu}=\partial_{\mu}-i g_{s} \sum_{a=1}^{8} \frac{\lambda_{\alpha \beta}^{a}}{2} G_{\mu}^{a}
$$

donde $g_{s}$ es la constante de acoplamiento de $S U(3)_{C}$ y suele escribirse como $\alpha_{s}=\frac{g_{s}^{2}}{4 \pi}$.

Se define el tensor de campo de Yang-Mills $G_{\mu \nu}^{a}$ para $S U(3)_{C}$ como:

$$
G_{\mu \nu}^{a}=\partial_{\mu} G_{\nu}^{a}-\partial_{\nu} G_{\mu}^{a}+g_{s} f_{a b c} G_{\mu}^{b} G_{\nu}^{c}
$$

donde $f_{a b c}$ son las constantes de estructura del grupo $S U(3)$. Es importante notar que el último término describe la auto interacción de los gluones, responsable de la naturaleza no abeliana de QCD.

Con esto se puede escribir la densidad lagrangiana de QCD como: 


$$
\mathcal{L}_{Q C D}=-\frac{1}{4} \sum_{a=1}^{8} G_{\mu \nu}^{a} G^{a \mu \nu}+\sum_{j=q u a r k s} \bar{q}_{j} i \gamma^{\mu} D_{\mu} q_{j}
$$

Dentro de la teoría cuántica de campos se encuentra que la constante de acoplamiento depende de la escala (Q) de la interacción. El cambio en una constante de acoplamiento producto de dicho cambio de escala en el que se trabaja, puede entenderse a partir de la acción del campo sobre las partículas que lleven la dada carga. El llamado screening en el vacío, producto de pares partícula-antipartícula virtuales que polarizan el vacío, modifican el valor efectivo de la carga dependiendo de la escala. Esta característica está presente tanto en QED como en QCD, pero este último presenta a su vez el hecho que los bosones de gauge transportan carga (de color en este caso) a diferencia de los fotones en QED. El efecto de polarización de los gluones virtuales es en la dirección contraria y tiende a aumentar la carga de color, lo que suele llamarse como anti-screening. El término dominante dependerá del número de "sabores activos" $n_{f}$ (es decir aquellos quarks con $m_{q}<Q$ ), y, por lo tanto, para el caso de QCD y en la aproximación a primer orden, la constante de acoplamiento puede escribirse como:

$$
\alpha_{s}(Q)=\frac{12 \pi}{\left(33-2 n_{f}\right) \cdot \log \left(\frac{Q^{2}}{\Lambda_{Q C D}^{2}}\right)}
$$

donde $\Lambda_{Q C D}^{2}$ es la escala del cut-off infra-rojo en la que la aproximación perturbativa ya no es válida.

De esta expresión se desprenden dos de las características más importantes de QCD, el confinamiento y la libertad asintótica. Para una escala de energía baja, cuando $Q^{2} \rightarrow 0, \alpha_{s}$ crece divergentemente, lo que indica que tanto los quarks como los gluones están confinados y no se pueden encontrar como partículas libres, sino que serán los constituyentes (que suelen llamarse partones) de objetos más complejos/no elementales: los hadrones. Por el contrario, en el límite de altas energías, $\alpha_{s}$ tiende a cero y se puede considerar a los quarks y gluones como partículas libres, lo que se conoce como libertad asintótica. En un colisionador de protones, los quarks y gluones producidos altas energías sufren un proceso conocido como hadronización, a medida que pierden energía, de manera que no se detectan quarks o gluones en el detector, sino que unos objetos conocidos como jets, que son un chorro de hadrones o una cascada de partículas, que forma un cono desde el quark/gluon inicial hasta el calorímetro. Estos efectos se detallan a continuación, luego de una breve descripción del proceso de renormalización.

\section{Renormalización}

Como se dijo, el SM es una teoría cuántica de campos renormalizable. En lo que sigue se detalla brevemente a qué refiere este término. Los efectos de órdenes superior introducen correcciones cuánticas, por ejemplo, en el cálculo de los acoplamientos en el SM, que deben tenerse en cuenta. Al mismo tiempo, las partículas en estos loops tienen momentos no acotados, por lo que surgen divergencias en los cálculos tanto para bajos momentos (llamadas infra-rojas o IR) como para altos momentos 
(ultra-violetas o UV), que deben eliminarse para que la teoría sea consistente con las mediciones experimentales. El proceso por el cual las divergencias desaparecen o se "absorben" al agregar una dependencia con la escala a los parámetros como los acoplamientos o masas de partículas, se conoce como renormalización. De esta forma el lagrangiano físico, con los acoplamientos comparables con los experimentos, se puede escribir como un lagrangiano bare o desnudo (a distancias tendiendo a cero), menos un lagrangiano que contenga los términos que eliminen las divergencias, a costo de introducir una dependencia con la escala $\mu$ del momento. Por tanto, la renormalización genera que los acoplamientos (y otros observables) no sean constantes y varíen con $\mu$. Tanto el screening en QED como la libertad asintótica y el confinamiento de QCD son consecuencias de este proceso de renormalización, que es a su vez una propiedad de las teorías de gauge.

\subsubsection{Interacciones hadrónicas en un colisionador protón- protón}

En el Capítulo 2, se hace una descripción detallada del detector ATLAS, con el cual se tomaron los datos utilizados para la búsqueda realizada en la presente tesis, y en la sección 5.2 se detallan las distintas muestras de simulaciones Monte Carlo que se usan para realizar la comparación naturaleza-teoría. Por su parte, en esta sección se describen las características generales de la descripción teórica de las interacciones entre protones en el colisionador.

Describir teóricamente y, consecuentemente, simular correctamente una colisión protón-protón es una tarea crucial y, al mismo tiempo, fuertemente compleja debido a la propia naturaleza de QCD y la dependencia con la energía de su acoplamiento. Se trata de la descripción de un proceso que involucra tanto el régimen perturbativo como el no perturbativo, es decir, interacciones entre partones (a cortas distancias o altas energías) y los hadrones y sus productos de decaimiento (bajas energías) que se miden en el detector. Este proceso de factorización lleva al tratamiento del evento separado en distintos regímenes de energía, lo que permite utilizar distintas herramientas de cálculo según corresponda.

El teorema de factorización [27], establece que la sección eficaz de producción de un proceso de QCD con dos partones A y B iniciales puede escribirse como:

$$
\sigma_{A B \rightarrow X}=\sum_{i, j} \int d x_{a_{i}} d x_{b_{j}} f_{A / a_{i}}\left(x_{a_{i}}, \mu_{F}^{2}\right) f_{B / b_{j}}\left(x_{b_{j}}, \mu_{F}^{2}\right) \sigma_{a_{i} b_{j} \rightarrow X}\left(\mu_{F}^{2}, \mu_{R}^{2}\right)
$$

donde $x_{i}$ y $x_{j}$ son las fracciones del momento de los hadrones $\mathrm{A}$ y $\mathrm{B}$, que llevan los partones $a_{i}$ y $b_{j}$, respectivamente, y $\sigma_{a_{i} b_{j}}$ es la sección eficaz de la interacción a nivel partónico, calculada a un dado orden de perturbaciones y una escala de renormalización $\mu_{R}$. Esta escala de normalización es importante para absorber las divergencias UV en los cálculos a órdenes mayores. Las funciones de la forma $f_{h / n}\left(x_{n}, \mu_{F}^{2}\right)$ son las PDFs, que representan la posibilidad de encontrar un partón de tipo $n$ en un hadrón $h$ con una fracción de momento $x_{n}$, dada la escala de factorización $\mu_{F}$. Esta escala es arbitraria y se introduce para tratar singularidades que aparecen en el regimen no perturbativo. Las PDFs no pueden ser determinadas perturbativamente, pero su dependencia funcional con la escala de energía se puede predecir con las ecuaciones 
DGLAP (Dokshitzer-Gribov-Lipatov-Altarelli-Parisi) [28, 29, 30]. En la presente tesis se consideran las predicciones teóricas a NLO utilizando las parametrizaciones CTEQ [31], MSTW [32, 33, 34] y NNPDF [35], a las que se vuelve en la Sección 6.1 cuando se discuten las incertezas sistemáticas teóricas.

Cuando dos protones colisionan a las altas energías de funcionamiento del LHC, los partones se encuentran en la condición de libertad asintótica, por lo que la interacción que se produce es efectivamente entre partones. El resultado de esta interacción evoluciona en el tiempo, como se detalla más adelante en esta sección, generando una cascada de partones hasta que la energía disponible es lo suficientemente baja como para que el confinamiento de QCD imponga la re-combinación de los partones en hadrones, proceso que no puede tratarse perturbativamente. La probabilidad de un partón en fragmentar en un determinado hadrón del estado final viene dada por las funciones de fragmentación $D_{c_{k}}$, que se introducen en la sección eficaz de la Ecuación 1.15 para obtener:

$$
\sigma_{a_{i} b_{j} \rightarrow C+X}=\int d z_{C} D_{c_{k}}\left(z_{C}, \mu_{f}^{2}\right) \sigma_{a_{i} b_{j} \rightarrow c_{k}+X}\left(\mu_{F}^{2}, \mu_{R}^{2}\right)
$$

donde $c_{k}$ es un partón con una dada probabilidad de fragmentar a un hadrón $C$, con una fracción $z_{C}$ de su momento, a la escala de fragmentación $\mu_{f}$.

Se tiene entonces, que las estimaciones teóricas y la correspondiente cadena de simulación para un evento de colisión p-p, como el que se muestra en la Figura 1.4, comienza con el cálculo del elemento de matriz $M$ de un dado proceso de dispersión dura o Hard Scatter (HS) entre los partones iniciales a distintos niveles en teoría de perturbaciones. El elemento de matriz contiene la información de la función de onda para los partones entrantes y salientes, y depende principalmente del acoplamiento fuerte y la escala de energía de la dispersión.

Debido a que los quarks son cargados bajo QCD y los gluones tienen acoplamientos triples y cuádruples, puede suceder que los partones emitan quarks o gluones antes de la dispersión dura, dando lugar a lo que se conoce como initial state radiation (ISR). De manera análoga, los partones salientes pueden emitir gluones o producir pares de quarks/anti-quarks y se tiene la final state radiation (FSR). A su vez, se pueden dar interacciones adicionales entre partones pertenecientes a los protones originales, que no participaron de la dispersión dura, dando lugar a múltiples interacciones en el evento (MPI), mayoritariamente interacciones de bajo momento (soft). La actividad no asociada con la dispersión dura se llama evento subyacente o underlying event (UE), que debido a la baja energía de las interacciones que lo componen, no puede ser tratado de manera perturbativa y se requieren de modelos fenomenológicos para describirlos.

La evolución de todos los partones producidos se realiza mediante un modelo de lluvia de partones (PS), que genera cascadas de radiación formadas por un gran número de gluones soft y quarks livianos, además de las partículas de la dispersión dura, hasta el límite de validez del régimen perturbativo, lo que se conoce como la escala de hadronización $\Lambda_{Q C D} \sim 1 \mathrm{GeV}$. Es importante mencionar en este punto que la combinación entre la dispersión dura y la lluvia partónica debe realizarse con especial cuidado de no duplicar en la PS los partones ya producidos y evitar así el doble conteo. Las estrategias principales para esto se conocen como CKKW (Catani-Krauss-Kuhn-Webber) [37, 38] y MLM [39]. 


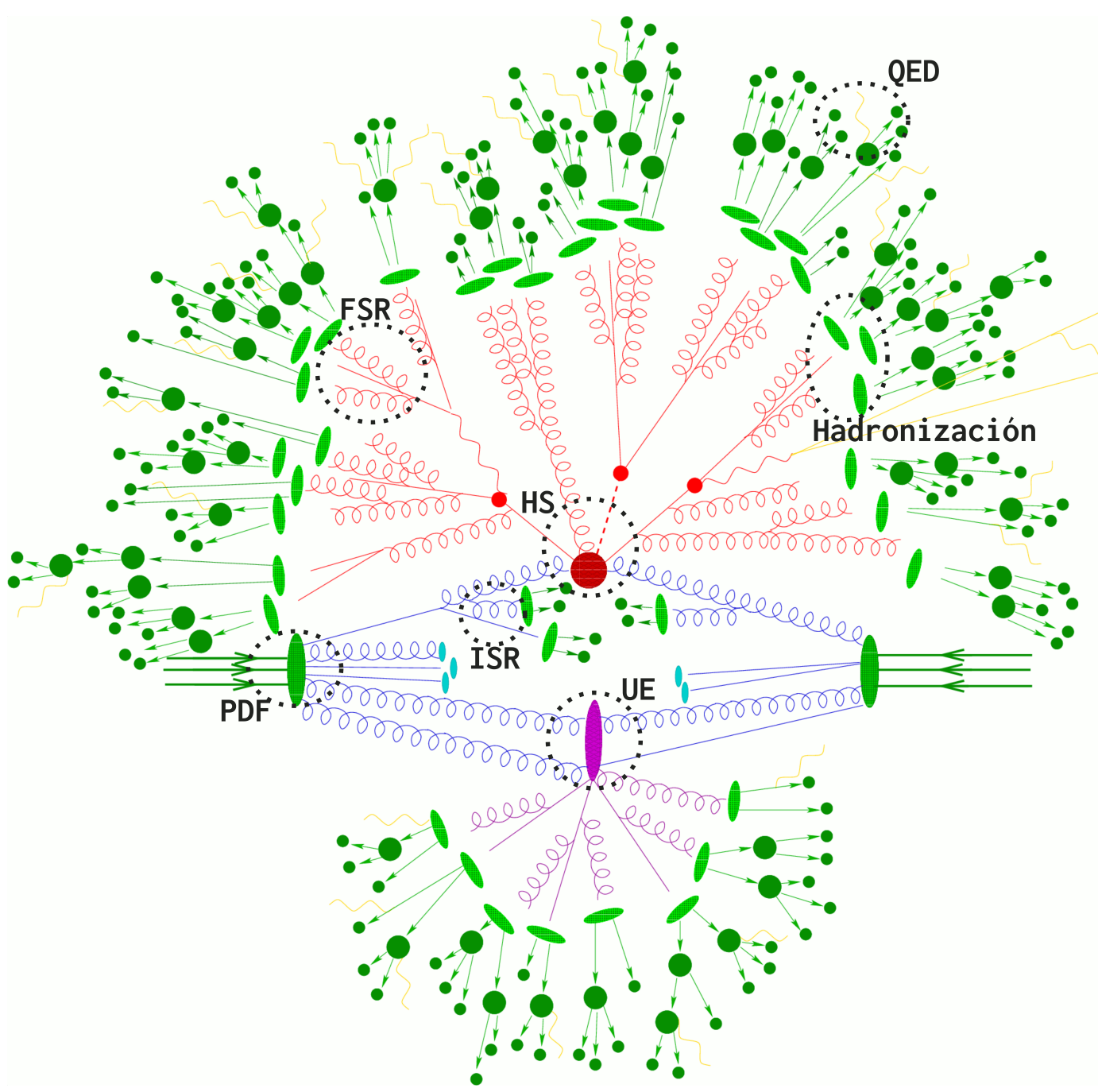

Figura 1.4: Esquema de una colisión protón-protón simulada por un generador de eventos de Monte-Carlo. La región roja en el centro representa la dispersión dura, rodeada por una estructura parecida a un árbol que representa al Bremsstrahlung como es simulado por los parton showers. La parte violeta indica un evento secundario de dispersión dura. Las transiciones de partones a hadrones están representadas por las partes verdes claras, mientras que las partes verdes oscuras indican el decaimiento de los hadrones. Finalmente, las líneas amarillas señalan la radiación de fotones [36]. 
Por último, la cadena termina con el proceso de hadronización, donde los partones en el estado final se recombinan para formar hadrones, de color neutro, debido a las bajas energías alcanzadas y el confinamiento de QCD. Estos hadrones, y el producto de sus decaimientos, se propagan a través del detector y pueden ser medidos experimentalmente. Para esto se cuenta con modelos fenomenológicos, ya que hasta el momento no hay forma de calcular su evolución en este régimen, debido a que no puede ser tratado perturbativamente.

\subsubsection{Limitaciones del Modelo Estándar}

El SM es el modelo teórico más exitoso que se haya construido, con un poder predictivo sin comparación y que desde aproximadamente la mitad del siglo veinte hacia adelante, fue motor y faro de los esfuerzos realizados por la comunidad de altas energía para el entendimiento más elemental de la naturaleza. Sin embargo, no puede considerarse la teoría final (aquella que podría "explicarlo todo"), ya que presenta ciertas limitaciones, tanto desde la mirada teórica como desde la experimental. De manera muy resumida se mencionan a continuación sólo algunas de estas limitaciones, para luego detallar una de ellas (divergencias cuadráticas), que se usa como disparador para nuevas teorías BSM.

- Problema de Jerarquía: Las escalas de la interacción débil y gravitatoria son extremadamente distintas, dando en particular $M_{W} / M_{P} \sim 10^{-17}$, y no hay una simetría que proteja a la escala electrodébil.

- Ruptura espontánea de la simetría electro-débil: Como se muestra en la Sección 1.2.2, se debe introducir de manera arbitraria un factor cuadrático en el potencial escalar para obtener el potencial con forma de sombrero mexicano.

- Masas de los neutrinos: Si bien durante la discusión del SM no se hizo hincapié en los neutrinos, el SM los considera como partículas no masivas, siendo que distintos experimentos detectaron su carácter masivo y le han puesto cotas a sus masas [40].

- Asimetría Bariónica: El universo que conocemos está compuesto de materia, cuando en principio no habría ninguna preferencia por ésta sobre por la antimateria. Este desbalance materia-antimateria que se observa en el universo no es explicado por el SM.

- Materia oscura: En base a mediciones astronómicas y consideraciones cosmológicas se sabe que la materia que conocemos es sólo el $4 \%$ del universo, mientras que un $23 \%$ del mismo se asocia a un tipo de materia que desconocemos y se la llama materia oscura, debido a que no emite radiación electromagnética, pero debe ser masiva producto de los efectos gravitacionales que tiene en la materia (visible). El SM no provee un candidato adecuado para materia oscura.

- No incluye gravedad.

\section{Divergencias cuadráticas}

El término de masa del Higgs renormalizado es su masa desnuda más correcciones de diagramas de orden superior: $\left(m_{h}^{2}\right)_{r}=\left(m_{h}^{2}\right)_{0}+\delta\left(m_{h}^{2}\right)$. Si se consideran sólo las 

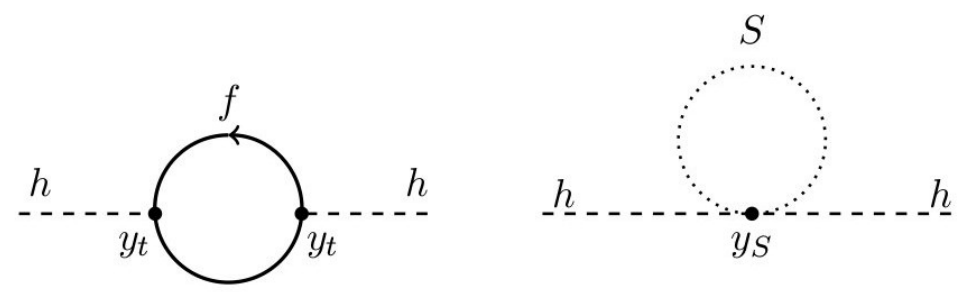

Figura 1.5: Divergencias cuadráticas: Corrección a un loop a $m_{h}^{2}$.

correcciones del quark top ${ }^{1}$ por ejemplo, se tiene que:

$$
\delta\left(m_{h}^{2}\right)_{f}=-\frac{y_{t}^{2}}{8 \pi^{2}}\left[\Lambda^{2}-3 m_{t}^{2} \ln \left(\frac{\Lambda^{2}}{m_{t}^{2}}\right)+\cdots\right]
$$

donde $\Lambda$ es la escala de energía a la que el SM como teoría efectiva deja de ser válido, y puede verse que la corrección anterior es cuadráticamente divergente en $\Lambda$.

Si se supone que no hay nueva física hasta la escala de GUT $\left(\Lambda \sim 10^{14}-10^{17}\right.$ $\mathrm{GeV})$ o la escala de Planck $\left(\Lambda=M_{P} \sim 10^{19} \mathrm{GeV}\right)$, entonces la masa desnuda del Higgs sería de $O(\Lambda)$,

$$
\left(m_{h}^{2}\right)_{0}=\left(m_{h}^{2}\right)_{r}-\delta\left(m_{h}^{2}\right)_{f} \simeq \Lambda^{2}\left(\frac{\left(m_{h}^{2}\right)_{r}}{\Lambda^{2}}-1\right) \simeq \Lambda^{2}\left(10^{-24}-1\right)
$$

donde $\Lambda=10^{14}$ y el acoplamiento de Yukawa para el top es de $O(1)$.

Esto introduce ciertos problemas, ya que $m_{h}$ parecería tener un valor en la escala de alta energía $\Lambda$, cuando la masa del Higgs observada $(125 \mathrm{GeV})$ es mucho menor que esta aparente escala "natural". Por lo tanto, para cancelar estas correcciones, habría que hacer un fine-tunning de los parámetros fundamentales en menos de una parte en $\sim 10^{12}$, lo que desde el punto de vista teórico parece ser en realidad falta de conocimiento de la física entre estas escalas.

Una forma de evitar este fine-tunning y al mismo tiempo cancelar las correcciones, podría darse si existiera un escalar complejo pesado $S$, con masa $m_{S}$ que se acople al Higgs con un término cuártico en el lagrangiano de la forma - $y_{S}\left(\phi_{S} \phi_{S}^{*}\right)\left(h h^{*}\right)$ (ver Figura 1.5), dado que entonces la corrección a un loop a la masa del Higgs sería:

$$
\delta\left(m_{h}^{2}\right)_{S}=\frac{y_{S}}{16 \pi^{2}}\left[\Lambda^{2}-m_{S}^{2} \ln \left(\frac{\Lambda^{2}}{m_{S}^{2}}\right)+\cdots\right]
$$

Esta contribución escalar es también cuadráticamente divergente, pero como es un loop bosónico, tiene signo opuesto comparado con la corrección a un loop fermiónico que se mostró antes. Para que ocurra una cancelación de las divergencias cuadráticas, la teoría debe tener dos escalares complejos que se acoplen al Higgs con $y_{S}=\left(y_{t}\right)^{2}$. Como se muestra a partir de la próxima sección, SUSY puede proveer las mencionadas nuevas partículas, agregando una nueva simetría entre fermiones y bosones.

\footnotetext{
${ }^{1}$ Es la más importante debido a que los acoplamientos de Yukawa son proporcionales a las masas de los fermiones.
} 


\subsection{Simetría Fermión-Bosón}

En el inicio de este capítulo se mencionó la importancia de las simetrías y su rol central en la formulación de las teorías que describen la naturaleza. Intentando continuar por este camino para extender el SM, la Supersimetría introduce una simetría entre las dos categorías de partículas constituyentes del SM, los fermiones y los bosones.

\subsubsection{El álgebra de SUSY}

Sea $Q$ el operador fermiónico que genera una transformación supersimétrica, convirtiendo un estado bosónico en uno fermiónico, y viceversa, tal que:

$$
\begin{aligned}
& Q \mid \text { Bosón }\rangle=\mid \text { Fermión }\rangle \\
& Q \mid \text { Fermión }\rangle=\mid \text { Bosón }\rangle
\end{aligned}
$$

Por lo tanto $Q$ es un espinor anticonmutativo y entonces $Q^{\dagger}$ es también generador de la simetría. Estos generadores llevan momento angular de espín 1/2, y satisfacen relaciones de anti-conmutación y conmutación de la forma:

$$
\begin{gathered}
\left\{Q, Q^{\dagger}\right\}=P^{\mu} \\
\{Q, Q\}=\left\{Q^{\dagger}, Q^{\dagger}\right\}=0 \\
{\left[P^{\mu}, Q\right]=\left[P^{\mu}, Q^{\dagger}\right]=0}
\end{gathered}
$$

donde $P^{\mu}$ es el momento generador de las traslaciones espacio-temporales. A estos modelos supersimétricos usualmente se los llaman SUSY $\mathrm{N}=1$, donde $\mathrm{N}$ se refiere al número de supersimetrías (o al número de distintas copias de $Q$ y $Q^{\dagger}$ ). Vale aclarar que ésta es la única excepción (al ser un generador fermiónico) al teorema de Coleman-Mandula para simetrías espacio-temporales [41].

\section{Supermultipletes}

Los estados de partícula de una teoría supersimétrica pertenecen a las representaciones irreducibles del álgebra de SUSY, llamadas supermultipletes. El operador de masa $-P^{2}$ conmuta con los generadores de la simetría, y con todos los operadores de rotación y traslación. Por consiguiente, los estados del supermutiplete deberán tener los mismos autovalores de $-P^{2}$, y entonces la misma masa. Además, como $Q$ y $Q^{\dagger}$ conmutan con los generadores de las transformaciones de gauge, se entiende que las partículas en un mismo supermultiplete deben tener la misma carga eléctrica, isospin y color.

Usualmente se dice que un fermión es el supercompañero de un bosón, y viceversa. Cada supermultiplete tiene que contener igual número de grados de libertad fermiónico y bosónico: $n_{B}=n_{F}$. Para lograr esto, las formas más simples (y a partir de las cuales formas más complejas pueden combinarse) son:

- Supermultiplete Quiral o de Materia o Escalar: Con un fermión de Weyl con dos estados de helicidad $\left(n_{F}=2\right)$ y dos escalares reales (cada uno con $n_{B}=1$ ) o uno complejo. 
- Supermultiplete de Gauge o Vectorial: Con un bosón de gauge vectorial de espín 1 no masivo (al menos antes del rompimiento) con dos estados de helicidad $\left(n_{B}=2\right)$, y un fermión no masivo de Weyl de espín $1 / 2$, con dos estados de helicidad $\left(n_{F}=2\right)$. Si se incluye la gravedad, el gravitón de espín 2 tiene un supercompañero de espín 3/2 llamado gravitino.

\subsection{El Modelo Estándar Supersimétrico Mínimo: MSSM}

El MSSM es la extensión al SM que requiere introducir la mínima cantidad de partículas que permita completar los supermultipletes quirales o de gauge (con diferencia de $1 / 2$ entre los espines de los supercompañeros).

Los quarks y leptones del SM deben acomodarse en supermultipletes quirales (debido a que sus partes izquierda y derecha transforman de forma diferente bajo el grupo de gauge) y sus supercompañeros de espín 0, serán los squarks y sleptones, para los cuales las interacciones de gauge de cada uno de los campos son las mismas que la de los correspondientes fermiones del SM.

El bosón escalar de Higgs debe estar en un supermultiplete quiral (tiene espín 0 ). En el SM el campo de Higgs es un doblete y de los cuatro grados de libertad, sólo permanece uno como consecuencia del rompimiento espontáneo de la simetría electrodébil, resultando el bosón de Higgs. En el MSSM se necesitan dos dobletes escalares complejos de Higgs, $H_{u}=\left(H_{u}^{+}, H_{u}^{0}\right)$ y $H_{d}=\left(H_{d}^{0}, H_{d}^{-}\right)$, lo que se conoce como un 2HDM (modelo de dos dobletes de Higgs). El escalar neutro que corresponde al Higgs del SM es entonces una combinación lineal de $H_{u}^{0}$ y $H_{d}^{0}$. Los supercompañeros de espín $1 / 2$ se llaman higgsinos: $\widetilde{H}_{u}$ y $\widetilde{H}_{d}$.

Por su parte, los bosones vectoriales del SM pertenecen a supermultipletes de gauge y sus supercompañeros fermiónicos llamados gauginos tienen espín 1/2. Para las interacciones de QCD, el octeto de gluones tiene el octeto supercompañero de gluinos. Los bosones $W^{+}, W^{0}, W^{-}$y $B^{0}$ de espín 1 asociados con la simetría electrodébil, tienen a los supercompañeros winos y bino (con igual símbolo agregando un $\sim$ ).

El espectro completo del MSSM se muestra en la Tabla 1.1.

\subsubsection{Rompimiento de Supersimetría}

Hasta el momento se ha omitido la discusión respecto a la no detección de ninguna spartícula. Si SUSY no estuviera rota, en base a la discusión anterior deberían existir, por ejemplo, selectrones con masa igual a $m_{e} \sim 511 \mathrm{keV}$. De manera análoga para el resto de los sfermiones y se deberían tener gluinos y fotinos sin masa. Sin embargo, ninguna compañera supersimétrica de las partículas del SM ha sido observada experimentalmente. Esto significa que SUSY debe ser a lo sumo una simetría rota en el estado de vacío. Pero, al mismo tiempo que no se ha encontrado experimentalmente ninguna spartícula, se necesita cierto "grado" de simetría para poder cancelar los términos de masas divergentes, como se menciona en la Sección 1.2.5. Se propone entonces un rompimiento soft en la teoría para que ésta sea compatible con las observaciones experimentales, sin dejar de resolver los problemas asociados en la teoría. Que el rompimiento sea soft implica que solo términos con dimensiones 


\begin{tabular}{|c|c|c|c|c|c|}
\hline Nombre & Símbolo & espín-0 & espín-1/2 & espín-1 & $S U(3)_{c}, S U(2)_{L}, S U(1)_{Y}$ \\
\hline \multicolumn{6}{|c|}{ Supermultipletes Quirales } \\
\hline squarks y quarks & $\mathrm{Q}$ & $(\widetilde{u}, \widetilde{d})_{L}$ & $(u, d)_{L}$ & & $\left(3,2, \frac{1}{3}\right)$ \\
\hline \multirow[t]{2}{*}{ (x3 familias) } & $\bar{u}$ & $\widetilde{u}_{R}^{*}$ & $u_{R}^{\dagger}$ & & $\left(\overline{3}, 1,-\frac{4}{3}\right)$ \\
\hline & $\bar{d}$ & $\tilde{d}_{R}^{*}$ & $d_{R}^{\dagger}$ & & $\left(\overline{3}, 1, \frac{2}{3}\right)$ \\
\hline \multirow{2}{*}{$\begin{array}{c}\text { sleptons y leptons } \\
\text { (x3 familias) }\end{array}$} & $\mathrm{L}$ & $(\widetilde{v}, \widetilde{e})_{L}$ & $(v, e)_{L}$ & & $(1,2,-1)$ \\
\hline & $\bar{e}$ & $\widetilde{e}_{R}^{*}$ & $e_{R}^{\dagger}$ & & $(1,1,2)$ \\
\hline \multirow{2}{*}{ higgs y higgsinos } & $H_{u}, \widetilde{\sim}_{u}$ & $\left(H_{u}^{+}, H_{u}^{0}\right)$ & $\left(\widetilde{H}_{u}^{+}, \widetilde{\sim}_{u}^{0}\right)$ & & $(1,2,1)$ \\
\hline & $H_{d}, \widetilde{H}_{d}$ & $\left(H_{d}^{0}, H_{d}^{-}\right)$ & $\left(\widetilde{H}_{d}^{0}, \widetilde{H}_{d}^{-}\right)$ & & $(1,2,-1)$ \\
\hline \multicolumn{6}{|c|}{ Supermultipletes de Gauge } \\
\hline gluino y gluon & & & $\widetilde{g}$ & $\mathrm{~g}$ & $(8,1,0)$ \\
\hline winos, $\mathrm{W}$ bosons & & & $\widetilde{W}^{ \pm} \widetilde{W}^{0}$ & $W^{ \pm} W^{0}$ & $(1,3,0)$ \\
\hline bino, B boson & & & $\widetilde{B}^{0}$ & $B^{0}$ & $(1,1,0)$ \\
\hline
\end{tabular}

Tabla 1.1: Espectro completo del MSSM.

de masa pueden romper SUSY y la relación de acoples se mantiene, permitiendo la cancelación de las divergencias cuadráticas (aunque exista una sensibilidad logarítmica).

El Lagrangiano del MSSM puede escribirse entonces como:

$$
\mathcal{L}_{M S S M}=\mathcal{L}_{S U S Y}+\mathcal{L}_{S O F T}
$$

separando la parte supersimétrica y el término arbitrario de rompimiento de la supersimetría. Ambas partes se describen con más detalle en lo que sigue.

\section{Término supersimétrico: $\mathcal{L}_{S U S Y}$}

El lagrangiano de SUSY tiene las mismas interacciones de gauge que el SM. Los acoplamientos de Yukawa fermión-escalar necesarios para dar masa a los quarks y a los leptones están contenidos en un superpotencial $W_{M S S M}$ que es una función de supermultipletes quirales:

$$
\mathrm{W}_{\text {MSSM }}=\mathrm{y}_{\mathrm{u}}^{\mathrm{ij}} \overline{\mathrm{u}}_{\mathrm{ai}} \mathrm{Q}_{\mathrm{j}}^{\mathrm{a}} \mathrm{H}_{\mathrm{u}}-\mathrm{y}_{\mathrm{d}}^{\mathrm{ij}} \overline{\mathrm{d}}_{\mathrm{ai}} \mathrm{Q}_{\mathrm{j}}^{\mathrm{a}} \mathrm{H}_{\mathrm{d}}-\mathrm{y}_{\mathrm{e}}^{\mathrm{ij}} \overline{\mathrm{e}}_{\mathrm{i}} \mathrm{L}_{\mathrm{j}} \mathrm{H}_{\mathrm{d}}+\mu \mathrm{H}_{\mathrm{u}} \mathrm{H}_{\mathrm{d}}
$$

Los primeros tres términos dan las masas para los leptones y los quarks up y down (sumados sobre los colores: $\mathrm{a}=1,2,3$ ). Los parámetros de acoplamiento de Yukawa (adimensionales) son matrices complejas de $3 \times 3$ en el espacio de familia $(i, j=1,2,3)$. Queda claro de esto, la necesidad de incluir dos dobletes de Higgs como se mencionó antes, para darle masa a todos los quarks y leptones. El término de $\mu$ es la versión supersimétrica del término de masa del bosón de Higgs en el SM.

\section{Término asociado al rompimiento soft de la simetría: $\mathcal{L}_{S O F T}$}

Como se verá en la Sección 1.5, existen diversos mecanismos que llevan al rompimiento de SUSY. Por lo tanto, el término $\mathcal{L}_{S O F T}$ queda supeditado al mecanismo particular que se utilice. De la forma más general, debe ser invariante frente a $S U(3)_{C} \times S U(2)_{L} \times U(1)_{Y}$ y soft. Entre los términos permitidos se encuentran: 
- Masas para los gauginos:

$$
\mathcal{L}_{\text {SOFT }} \supset-\frac{1}{2}\left(\mathrm{M}_{1} \widetilde{\mathrm{BB}} \widetilde{\mathrm{B}}+\mathrm{M}_{2} \widetilde{\mathrm{W}}^{\mathrm{b}} \widetilde{\mathrm{W}}^{\mathrm{b}}+\mathrm{M}_{3} \widetilde{\mathrm{g}}^{\mathrm{a}} \widetilde{\mathrm{g}}^{\mathrm{a}}+\text { h.c. }\right)
$$

- Interacciones trilineares para los escalares y los higgses (A-terms):

$$
\mathcal{L}_{\text {SOFT }} \supset-\left(\mathrm{a}_{\mathrm{u}}^{\mathrm{ij}} \widetilde{\mathrm{u}}_{\mathrm{ai}} \widetilde{\mathrm{Q}}_{\mathrm{j}}^{\mathrm{a}} \mathrm{H}_{\mathrm{u}}-\mathrm{a}_{\mathrm{d}}^{\mathrm{ij}} \widetilde{\mathrm{d}}_{\mathrm{ai}} \widetilde{\mathrm{Q}}_{\mathrm{j}}^{\mathrm{a}} \mathrm{H}_{\mathrm{d}}-\mathrm{a}_{\mathrm{e}}^{\mathrm{ij}} \widetilde{\mathrm{e}}_{\mathrm{i}} \widetilde{\mathrm{L}}_{\mathrm{j}} \mathrm{H}_{\mathrm{d}}+\text { h.c. }\right)
$$

- Masas del sector de Higgs y términos de mezcla escalar bilineares (B-terms):

$$
\mathcal{L}_{\text {SOFT }} \supset-\mathrm{m}_{\mathrm{H}_{\mathrm{u}}}^{2} \mathrm{H}_{\mathrm{u}}^{*} \mathrm{H}_{\mathrm{u}}-\mathrm{m}_{\mathrm{H}_{\mathrm{d}}}^{2} \mathrm{H}_{\mathrm{d}}^{*} \mathrm{H}_{\mathrm{d}}-\left(\mathrm{bH}_{\mathrm{u}} \mathrm{H}_{\mathrm{d}}+\text { h.c. }\right)
$$

- Términos de masa para los sfermiones:

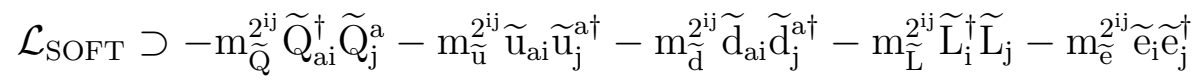

En secciones subsiguientes se realiza una discusión más extendida sobre el rompimiento de SUSY, donde se describen dos de las propuestas más importantes para las interacciones mediadoras.

\subsubsection{Parámetros libres de la teoría}

Cuando se introduce en el lagrangiano el término de ruptura de SUSY, se incorporan una serie de nuevos parámetros libres a la teoría que están relacionados como sigue. Tres nuevos parámetros $M_{1}, M_{2}$ y $M_{3}$ están asociados con las masas soft para los gauginos. A su vez, los acoplamientos trilineares para los squarks y sleptons agregan $a_{u}, a_{d}$ y $a_{e}$, mientras que las masas soft del sector de Higgs y el parámetro de la interacción escalar bilinear suman $m_{H_{u}}, m_{H_{d}}$ y $b$. Por último, las masas soft de los squarks y sleptones añaden $\mathrm{m}_{\widetilde{\mathrm{Q}}}, \mathrm{m}_{\widetilde{\mathrm{u}}}, \mathrm{m}_{\widetilde{\mathrm{d}}}, \mathrm{m}_{\widetilde{\mathrm{L}}} \mathrm{y} \mathrm{m}_{\tilde{\mathrm{e}}}$.

Por lo tanto, si se tiene un modelo con una sola familia de quarks, leptones y sus supercompañeros, el MSSM tendría 14 parámetros nuevos. Mientras que en un modelo completo de tres familias, los parámetros de masa de los squarks y sleptones, y los parámetros A, serán matrices de 3x3, sumado a la posibilidad de mezcla entre familias. No obstante, como no todos los parámetros son físicos, el número puede reducirse hasta obtener 124 parámetros libres para el MSSM de los cuales:

- 18 corresponden al SM.

- 1 al sector de Higgs.

- 105 nuevos parámetros del modelo.

Si la cantidad de parámetros libres del SM era ya una cantidad importante, el agregar del orden de 100 parámetros nuevos implica (casi como única alternativa) que, con el fin de disminuir los grados de libertad, se deben llevar a cabo fuertes suposiciones a fin de acotar los valores de varios de sus parámetros. 


\subsubsection{Paridad $\mathrm{R}$}

Los términos incluidos en el superpotencial del MSSM (Ec. 1.17) no son los únicos posibles, y se pueden agregar otros términos invariantes de gauge como:

$$
\begin{aligned}
W_{\Delta \mathrm{L}=1} & =\frac{1}{2} \lambda^{i j k} L_{i} L_{j} \bar{e}_{k}+\lambda^{\prime i j k} L_{i} Q_{j} \bar{d}_{k}+\mu^{\prime i} L_{i} H_{u} \\
W_{\Delta \mathrm{B}=1} & =\frac{1}{2} \lambda^{\prime \prime i j k} \bar{u}_{i} \bar{d}_{j} \bar{d}_{k}
\end{aligned}
$$

Sin embargo, estos términos son fenomenológicamente indeseados ya que, a diferencia de los anteriores mencionados y los del lagrangiano del SM, no conservan el número leptónico (L) y bariónico (B). En particular, el primero de la Ecuación 1.18 viola el número leptónico en una unidad, mientras que el segundo hace lo mismo con el número bariónico (esto se especifica en los subíndices de $W$ ). De estar presentes estos términos, el protón podría decaer, por ejemplo, a un positrón y a un pión neutro mediado por un squark supercompañero escalar del quark d. Sin embargo, el límite experimental para la vida media del protón es $>10^{33}$ años [42], por lo que los acoplamientos $\lambda$ deben ser muy pequeños, o se debe postular la conservación de L y B en el MSSM. Para esto último, en el MSSM se introduce una nueva simetría que prohíbe la presencia de estos términos en el superpotencial y evitar así la posibilidad del decaimiento del protón. Dicha simetría se conoce como paridad R, y se define como:

$$
P_{R}=(-1)^{3(B-L)+2 S},
$$

donde además de los números bariónico y leptónico, se tiene en cuenta el espín (S) de la partícula. Se tiene entonces que las partículas del SM tendrán $P_{R}=1$ y las spartículas $P_{R}=-1$.

La inclusión de esta nueva simetría tiene consecuencias fenomenológicas importantes. Como las partículas del SM tienen opuesto valor de $P_{R}$ a las spartículas, no puede haber mezcla entre ellas y en cualquier vértice debe haber un número par de spartículas. A su vez, la partícula supersimétrica más liviana (LSP) debe ser estable y cada spartícula, diferente de la LSP, debe decaer eventualmente a un estado con número impar de LSPs. Si la LSP es eléctricamente neutra y solo interactúa débilmente con la materia ordinaria, puede ser una buena candidata para materia oscura. Finalmente, debido a la gran influencia que tienen en la búsqueda que se realiza en la presente tesis, vale destacar que en experimentos colisionadores, la producción de spartículas se da de a pares, en general de a 2, y debido a la estabilidad de la LSP los estados finales tendrán energía transversa faltante.

\subsubsection{El espectro de masa del MSSM}

Es necesario remarcar que no todas las partículas que se muestran en la Tabla 1.1 son autoestados de masa de la teoría. Esto se debe a que, después del rompimiento de la simetría electrodébil y de la supersimetría, pueden darse mezclas entre los gauginos y los higgsinos, y entre partículas/spartículas con mismos números cuánticos.

A continuación se presenta un detalle de las posibles mezclas que pueden ocurrir y que producen el espectro de masas del MSSM: 
- Squarks y sleptons

Se puede producir mezcla entre cualquier par de escalares con la misma carga eléctrica, paridad-R y color. Los autoestados de masa de los squarks y sleptones se obtienen diagonalizando tres matrices de masa de 6x6 para los squarks tipo up $\left(\widetilde{u}_{L}, \widetilde{c}_{L}, \widetilde{t}_{L}, \widetilde{u}_{R}, \widetilde{c}_{R}, \widetilde{t}_{R}\right)$, squarks tipo down $\left(\widetilde{d}_{L}, \widetilde{s}_{L}, \widetilde{b}_{L}, \widetilde{d}_{R}, \widetilde{s}_{R}, \widetilde{b}_{R}\right)$, y sleptones cargados $\left(\widetilde{e}_{L}, \widetilde{\mu}_{L}, \widetilde{\tau}_{L}, \widetilde{e}_{R}, \widetilde{\mu}_{R}, \widetilde{\tau}_{R}\right)$, y una matriz de $3 \mathrm{x} 3$ para sneutrinos $\left(\widetilde{\nu}_{e}, \widetilde{\nu}_{\mu}, \widetilde{\nu}_{\tau}\right)$.

\section{- Gluinos}

El gluino no puede mezclarse con ninguna otra partícula del MSSM debido a que es un fermión de color de ocho componentes. La fuente para la masa del gluino es el término que rompe a SUSY, incluido en $\mathcal{L}_{S O F T}$, cuyo parámetro de masa es $M_{3}$.

\section{- Charginos}

Los charginos $\widetilde{\chi}_{1,2}^{ \pm}$son fermiones de Dirac de cuatro componentes, mezcla de los higgsinos y gauginos cargados eléctricamente $\left(\widetilde{H}_{u}^{+}, \widetilde{H}_{d}^{-}\right.$y $\left.\widetilde{W}^{+}, \widetilde{W}^{-}\right)$, debido al rompimiento de la simetría electrodébil. Para determinar los estados físicos y las masas de los charginos, se debe diagonalizar la matriz de masas dependiente de $M_{2}, \mu$ y $\beta$. Por convención se toma $m_{\widetilde{\chi}_{1}^{ \pm}} \leq m_{\widetilde{\chi}_{2}^{ \pm}}$.

\section{- Neutralinos}

Los neutralinos $\widetilde{\chi}_{i}^{0}($ con $i=1,2,3,4)$ son fermiones de Majorana mezcla de $\widetilde{B}$, $\widetilde{W}^{0}, \widetilde{H}_{u}^{0}$ y $\widetilde{H}_{d}^{0}$. Su matriz de masa [43] depende de los parámetros $M_{1}, M_{2}, \mu$ y $\tan (\beta)$. El neutralino más liviano, $\widetilde{\chi}_{1}^{0}$, se suele considerar como la LSP, ya que es una de las partículas supersimétricas lo suficientemente liviana para ser una buena candidata a materia oscura.

\section{- Sector de Higgs}

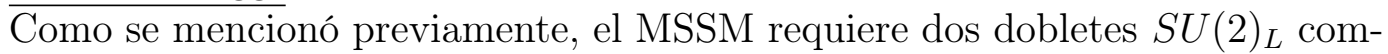
plejos $H_{u}$ y $H_{d}$ para el sector de Higgs, lo que provee de ocho grados de libertad escalares. Después del rompimiento de la simetría electrodébil, tres de estos son bosones de Nambu-Goldstone que se convierten en los modos longitudinales de los bosones vectoriales $Z$ y $W^{ \pm}$.

Los cinco restantes producen los bosones escalares de Higgs físicos del modelo (si se supone un potencial invariante bajo $\mathrm{CP}$ ):

- Un par de bosones cargados $H^{ \pm}: m_{H^{ \pm}}^{2}=m_{A^{0}}^{2}+m_{W}^{2}$

- Un bosón neutral CP-impar $A^{0}: m_{A^{0}}^{2}=\frac{2 b}{\sin (2 \beta)}$

- Y los bosones neutrales CP-par $H^{0}$ y $h^{0}$ :

$$
m_{h^{0}, H^{0}}^{2}=\frac{1}{2}\left(m_{A^{0}}^{2}+m_{Z}^{2} \mp \sqrt{\left(m_{A^{0}}^{2}-m_{Z}^{2}\right)^{2}+4 m_{Z}^{2} m_{A^{0}}^{2} \sin ^{2}(2 \beta)}\right)
$$

Por convención, $h^{0}$ es el bosón más liviano y se comporta similar al del SM. Su masa a nivel árbol es del órden de la masa del bosón $Z$, pero tiene grandes correcciones a nivel loop. 


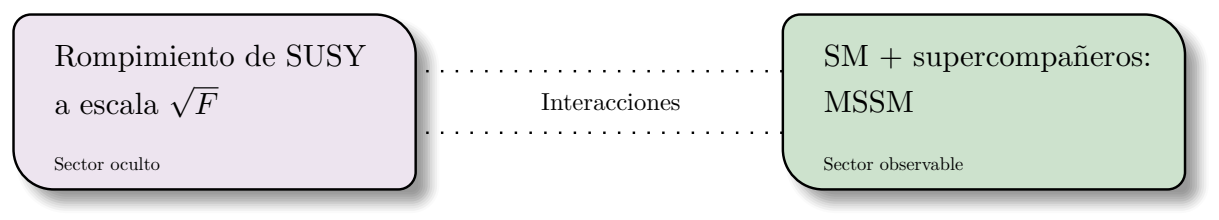

Figura 1.6: Ruptura de SUSY. Se extiende el MSSM con un sector oculto donde se rompe SUSY. Las partículas pertenecientes a este nuevo sector no tienen acoplamientos con las partículas del MSSM. Las interacciones que median entre ambos sectores pueden ser de distinta naturaleza, permitiendo una gran variedad de modelos que intentan explicar el rompimiento.

\subsection{Modelos para el mecanismo de rompimiento de SUSY}

En la Sección 1.4.1 se concluyó que SUSY, de existir, debe estar rota y, en particular, el rompimiento debe ser soft para mantener la solución a las divergencias cuadráticas.

El rompimiento de una simetría global genera un modo no masivo de NambuGoldstone con los mismos números cuánticos que el generador de la simetría rota. Para el caso de la Supersimetría global, el generador es la carga fermiónica $Q_{\alpha}$, por lo que la partícula de Nambu-Goldstone será un fermión de Weyl no masivo neutro, el goldstino (el cual es absorbido como modo longitudinal del gravitino, en un mecanismo que se conoce como Super Higgs).

El rompimiento espontáneo de SUSY requiere la extensión del MSSM. Se agrega entonces un sector oculto, de partículas sin acoplamientos directos (o muy pequeños) con los supermultipletes quirales (incluidas en el sector observable o visible) del MSSM. Estos dos sectores comparten interacciones, que median el rompimiento de SUSY desde el sector oculto al observable, dando los términos soft del lagrangiano del MSSM. En la Figura 1.6, se puede ver esquemáticamente esta idea.

Las interacciones mediadoras entre el sector oculto y el observable pueden ser de distinta naturaleza, por lo que existen muchos modelos que intentan explicar de esta forma el rompimiento de SUSY. A continuación se hace una breve descripción de algunas de las propuestas existentes.

\subsubsection{Propuestas para las interacciones mediadoras}

\section{Interacciones gravitacionales}

Si las interacciones son gravitacionales, los modelos se enmarcan en lo que se conoce como Planck scale mediated supersymmetry breaking o PMSB, debido a que la gravedad entra cerca de la escala de Planck. Si SUSY se rompe en el sector oculto por un vev $\langle F\rangle$, entonces los términos soft en el sector visible serán:

$$
m_{\text {soft }} \sim \frac{<F>}{M_{P}}
$$


Para $m_{\text {soft }}$ del orden de los cientos de GeV, la escala de rompimiento de SUSY en el sector oculto es $\sqrt{\langle F\rangle} \sim 10^{11} \mathrm{GeV}$.

Cuando se tiene en cuenta la gravedad, SUSY deber ser una simetría local y la teoría se conoce como supergravedad. En este caso, el gravitón de espín 2 tiene un supercompañero fermión de espín 3/2, el gravitino, inicialmente no masivos. Una vez que SUSY es espontáneamente rota, el gravitino absorbe el goldstino, adquiriendo masa, que se convierte en sus componentes longitudinales (helicidad $\pm 1 / 2$ ). La masa del gravitino $m_{3 / 2}$, se puede estimar de la Ec. 1.20, y se espera que sea comparable a la masa de las partículas del MSSM $\sim 100 / 1000 \mathrm{GeV}$.

\section{Modelos con mediadores de gauge}

Si se considera interacciones de gauge electrodébiles y QCD ordinarias, se tienen los modelos Gauge Mediated Supersymmetry Breaking o GMSB [10, 11, 12] (se considera aquí que estas interacciones siempre dominan sobre gravedad). Los términos soft del MSSM provienen de diagramas a un loop que involucran partículas mensajeras, que son nuevos supermultipletes quirales que se acoplan al vev $\langle F\rangle$ que rompe SUSY, y tienen a su vez interacciones $S U(3)_{C} \times S U(2)_{L} \times U(1)_{Y}$ que generan la conexión con el MSSM. Se tiene en este caso:

$$
m_{\text {soft }} \sim \frac{\alpha_{i}}{4 \pi} \frac{<F>}{M_{\text {mens }}}
$$

donde $M_{\text {mens }}$ es la escala característica de las masas de los campos mensajeros. En caso que $M_{\text {mens }}$ y $\sqrt{\langle F\rangle}$ sean comparables, se pueden tener $m_{\text {soft }}$ en el correcto orden de magnitud con sólo $\sqrt{\langle F\rangle} \sim 10^{4} \mathrm{GeV}$.

El marco más general se conoce como GGM (General Gauge Mediation), en el cual se define al mecanismo de mediación por campos de gauge como el límite en que las constantes de acoplamiento del MSSM $\alpha_{i} \rightarrow 0$, la teoría se desacopla en el MSSM y un sector oculto separado que rompe SUSY.

El conjunto de parámetros independientes está compuesto por las tres masas complejas de gauginos $M_{1}, M_{2}$ y $M_{3}$, y tres parámetros reales que controlan las masas de los cinco fermiones $m_{Q, u, d, L, e}^{2}$. Como característica principal, la masa del gravitino es: $m_{3 / 2}<<M_{W}$, típicamente del orden del $\mathrm{eV}$, lo que implica que es la LSP de la teoría. Es interesante notar que debido a que la LSP es siempre el gravitino, la partícula más liviana del MSSM es la NLSP de la teoría y su naturaleza determina entonces el estado final que se encuentra en un colisionador donde, debido a que el gravitino siempre escapa el detector, se espera que haya energía transversa faltante.

En la búsqueda que se lleva a cabo en la presente tesis, la NLSP es el neutralino más liviano, $\widetilde{\chi}_{1}^{0}$. Como se mencionó en la Sección 1.4.4, los neutralinos son mezcla del bino, winos y higgsinos, y dependiendo de su composición se tienen diferentes estados finales. Por lo tanto, el neutralino decae al $\widetilde{G}$ LSP y a $\gamma, Z$ o $h$. Cuando el neutralino es bino puro, la probabilidad de decaimiento (o BR) es mayoritariamente a $\gamma$, si es wino puro decae principalmente a $Z$ y si es higgsino puro, dependiendo de los valores de $\tan (\beta)$ y $\mu$, el higgsino decae a $\mathrm{Z}$ o a h o a una mezcla de ambos.

En el caso que se trata en el Capítulo 5 , se considera un neutralino mezcla de bino y higgsino con el parámetro $\mu>0$, donde los decaimientos dominantes son $\widetilde{\chi}_{1}^{0} \rightarrow \gamma \widetilde{G}$ y $\widetilde{\chi}_{1}^{0} \rightarrow Z \widetilde{G}$, con el decaimiento al bosón de Higgs suprimido, dando un 
estado final con al menos un fotón, jets (provenientes del Z) y energía transversa faltante (proveniente del gravitino) [1, 2, 44].

Cada una de las diferentes posibilidades para la composición del neutralino NLSP antes mencionadas, conforman un análisis distinto y dedicado de ATLAS. Vale mencionar entonces los otros análisis con fotones que completan los escenarios provistos por modelos GGM. Búsquedas con un fotón y leptones [45, 46], con di-fotones [47] y con un neutralino mezcla de bino y higgsino con $\mu<0$, dando un fotón y higgs (donde este último decae a dos b-jets) [45].

\subsubsection{Stealth SUSY}

Dentro de la colaboración ATLAS, se han (y se siguen) realizado enormes esfuerzos para cubrir de forma lo más completa posible, todo el espacio de parámetros y los estados finales disponibles que deriven (principalmente) del MSSM y modelos simplificados de SUSY. Una característica que suelen compartir estas búsquedas, es la presencia de un alto momento transverso faltante $\left(E_{\mathrm{T}}^{\mathrm{miss}}\right)$ en el estado final, dejando huecos sin analizar en el espacio de fase, que bien podría "usar SUSY para esconderse". La razón para que estados finales con bajo momento transverso faltante no sean los primeros analizados está lejos de ser arbitraria, y se basa en la alta complejidad experimental que presentan y en las propias restricciones teóricas. Entre los principales modelos que servirían a este propósito dentro del MSSM, se pueden mencionar aquellos con un espectro comprimido o modelos con largas cadenas de decaimiento, que por su propia naturaleza requieren una LSP que provea al evento bajo $E_{\mathrm{T}}^{\text {miss }}$. Por otro lado, si se logra justificar el no decaimiento del protón por algún dado mecanismo, se pueden estudiar modelos con violación de la paridad $\mathrm{R}$, donde el bajo $E_{\mathrm{T}}^{\text {miss }}$ proviene de la posibilidad de las spartículas de decaer a partículas del SM, siendo entonces visibles al detector y reduciendo así el momento transverso perdido en el estado final.

Una opción extra que surge y brinda eventos con estados finales con bajo MET, y al mismo tiempo con una potencial buena discriminación señal/fondo, son los modelos de Stealth SUSY [48, 49]. Este tipo de modelos se basa en extender el MSSM, con la inclusión de un nuevo sector, llamado sector de Stealth. Este nuevo sector está acoplado débilmente al MSSM, pero está desacoplado del sector de rompimiento de SUSY (generando la aproximada degeneración de masa en estas nuevas partículas), como se ilustra en la Figura 1.7.

En su forma más simple, este nuevo sector está compuesto por sólo dos nuevas partículas, el singlino $(\widetilde{S})$ y el singlete $(S)$, cuya diferencia de masas es pequeña, usualmente $\sim 10 \%$.

Dos de los decaimientos posibles que se puede obtener de este tipo de modelos, se muestran en la Figura 1.8. A la izquierda se tiene el caso más simple, donde un gluino decae a un jet y al singlino, que a su vez decae al singlete (éste decae a dos jets) y al gravitino LSP. Como la diferencia de masas entre el singlino y el singlete es pequeña, el MET que se obtiene es bajo como se buscaba. A la derecha, se muestra uno de los decaimientos que se explicará y analizará con detalle en el Capítulo 7, donde un squark decae al neutralino NLSP, siendo en este caso bino puro, que decae a un fotón y a un singlino, quien continua la cadena como el caso anterior. Este caso es particularmente interesante ya que, además de contar con bajo MET, se cuenta con dos fotones en el estado final, piezas claves para generar una reducción 


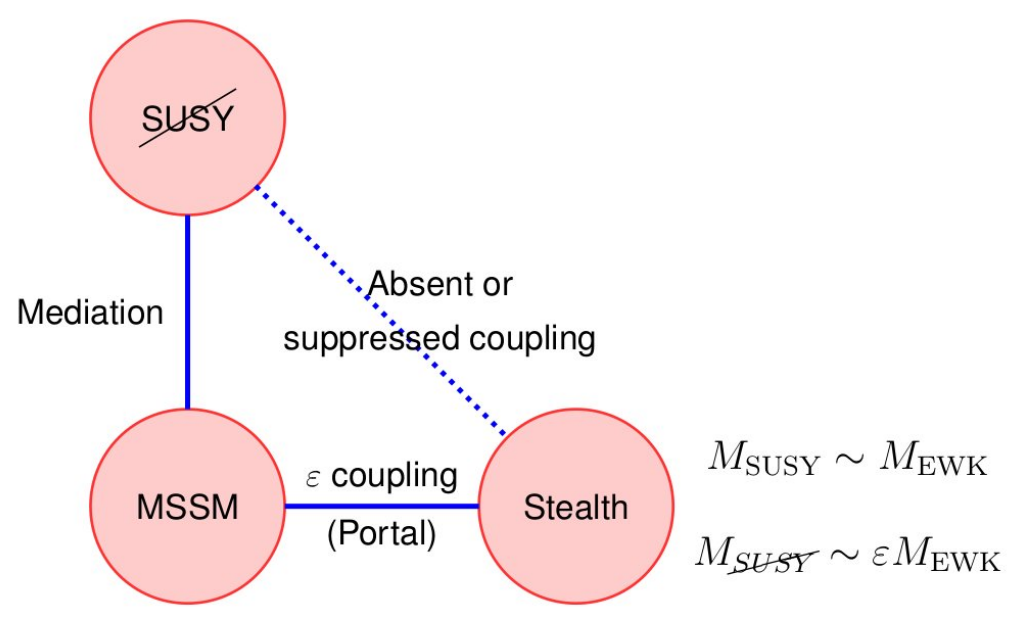

Figura 1.7: Mecanismo de Stealth.

sustancial del fondo del SM.

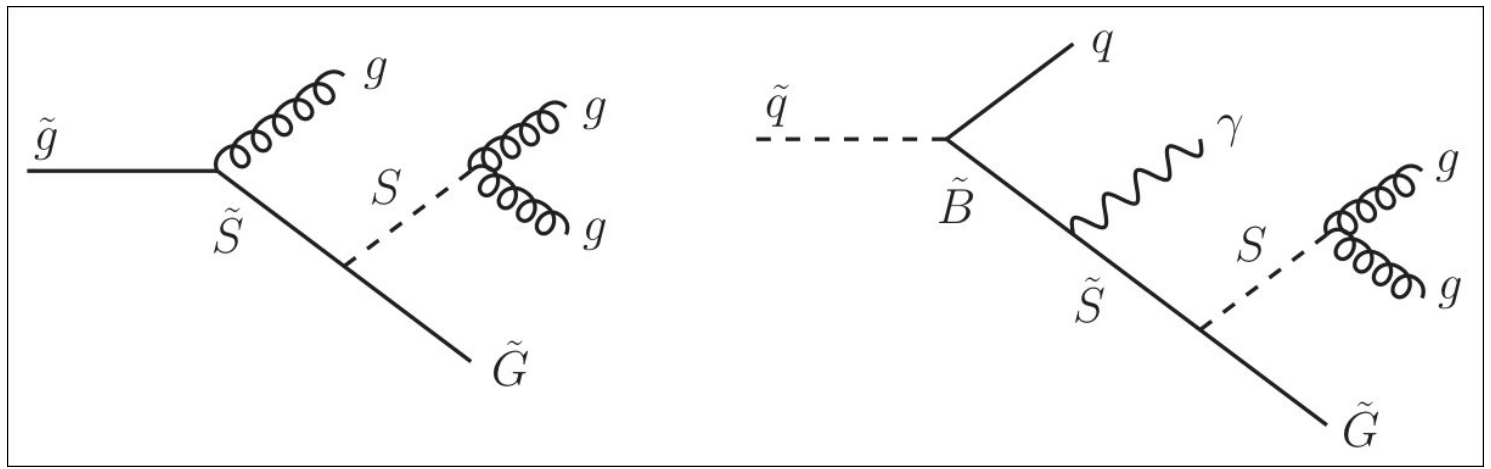

Figura 1.8: Decaimientos posibles de modelos Stealth. 


\section{Capítulo 2}

\section{El experimento ATLAS}

En este capítulo se introduce al experimento ATLAS, dentro del LHC. Se describe, inicialmente, el complejo de aceleradores y los parámetros más importantes de un colisionador hadrónico. Luego, la descripción del detector recorre todos sus subdetectores y el sistema de trigger, haciendo hincapié sobre todo en este último y en los calorímetros, debido al rol fundamental que ocupan en el desarrollo de esta tesis. Finalmente, se detalla el manejo y distribución de datos (tarea no trivial cuando se trabaja con $\sim$ Peta Bytes/año) y se hace una descripción de la toma de datos durante el Run-2.

\section{1. $\quad$ El Gran Colisionador de Hadrones}

El Gran Colisionador de Hadrones (Large Hadron Collider (LHC)) [50] es un acelerador de hadrones superconductor de dos anillos, perteneciente a la Organización Europea para la Investigación Nuclear (CERN), ubicado en las afueras de la ciudad de Ginebra, Suiza. Este sincrotrón de $27 \mathrm{~km}$ de circunferencia fue construido en el mismo túnel en el que funcionaba el acelerador $e^{+} e^{-}$LEP (Large Electron-Proton Collider) [51], a una profundidad variable entre 50 y 174 metros de la superficie.

El LHC fue diseñado para colisionar protones, a una energía de centro de masa de $\sqrt{s}=14 \mathrm{TeV}$, e iones pesados ${ }^{1}$ a $2.76 \mathrm{TeV}$ por nucleón, en cuatro puntos de interacción donde se alojan los cuatro detectores más importantes: ATLAS [52], CMS [53], ALICE [54] y LHCb [55]. Se define la energía del centro de masa $(\sqrt{s})$ como la cantidad total de energía del sistema protón-protón en el punto de interacción desde el sistema de laboratorio. El complejo de aceleradores y experimentos del LHC se muestra en la Figura 2.1, donde los puntos amarillos sobre el anillo más grande representan a los cuatro grandes experimentos antes mencionados, y se puede apreciar la cadena con los distintos pre-aceleradores por los que transitan los protones antes de llegar al LHC. Esta cadena se inicia cuando un paquete de protones (que se conoce como bunch), compuesto por $\sim 10^{11}$ protones obtenidos a partir de ionizar (y descartar los electrones) átomos de hidrógeno, ingresan en el único acelerador lineal de la cadena, el Linac 2. Este paquete sale con una energía de hasta $50 \mathrm{MeV}$ e ingresa al Proton Synchroton Booster, el primer acelerador circular, con $160 \mathrm{~m}$ de circunferencia, que permite acelerar los protones hasta $1.4 \mathrm{GeV}$. El proceso continua con una aceleración adicional provista por el Proton Synchroton,

\footnotetext{
${ }^{1} \mathrm{~A}$ menos que se explicite, para el resto de la tesis se considerarán colisiones entre protones.
} 


\section{CERN's Accelerator Complex}

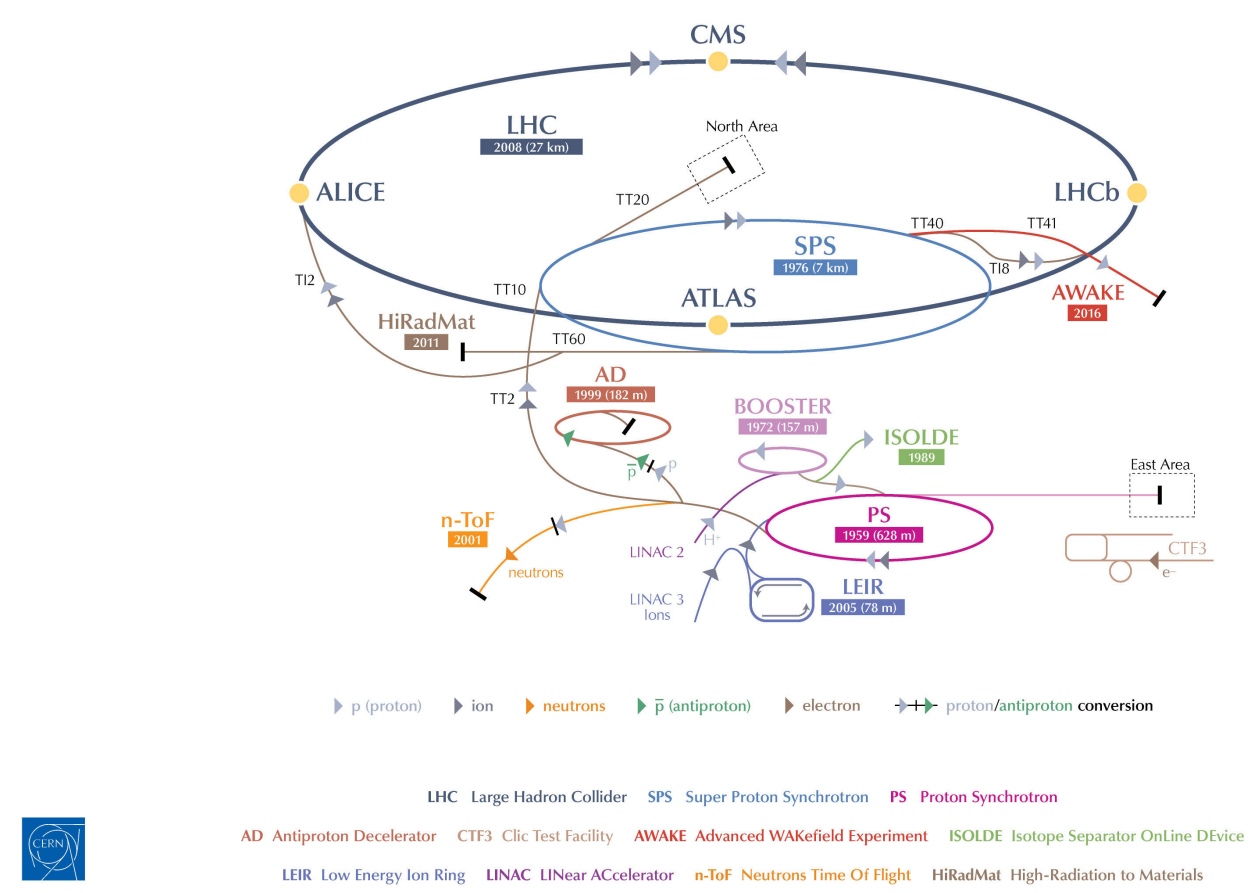

Figura 2.1: Complejo de aceleradores del CERN, incluyendo al LHC y a la serie de aceleradores utilizados para proveer de protones al LHC. También pueden verse los diferentes experimentos ubicados en distintos puntos del acelerador.

cuya circunferencia es de $630 \mathrm{~m}$, dejando a los protones con una energía de hasta $25 \mathrm{GeV}$. En el último paso antes de ingresar en el LHC, los protones pasan por el Super Proton Synchroton, que tiene $7 \mathrm{~km}$ de circunferencia e inyecta protones de $450 \mathrm{GeV}$ a cada uno de los anillos del LHC. La aceleración final se da en el propio LHC utilizando dos cavidades de radio frecuencia, hasta finalmente contar con dos haces de protones girando en direcciones contrarias con energías de $6.5 \mathrm{GeV}$ por haz, en dos anillos (distintos) con ultra alto vacío a una presión de $10^{-10}$ torr. El LHC cuenta con 1232 dipolos magnéticos superconductores que operan a $1.9 \mathrm{~K}$, y pueden generar un campo magnético con picos de $8.4 \mathrm{~T}$, manteniendo en su órbita circular a los protones. Los dipolos están equipados con sextupolos, octupolos y decapolos, que permiten corregir las pequeñas imperfecciones (y sus respectivas desviaciones) del campo magnético en las extremidades de los dipolos. Para aumentar la probabilidad de colisión, existe un sistema de focalización de los haces en las proximidades de los detectores que estrecha el camino que recorren los protones. El mismo consiste de 392 cuadrupolos magnéticos que generan campos magnéticos de $6.8 \mathrm{~T}$.

El diseño del LHC permite almacenar hasta 3564 bunches por anillo, pero el número efectivo es de $2808\left(n_{b}\right)$. Los bunches se encuentran espaciados temporalmente en $25 \mathrm{~ns}$ y su frecuencia de revolución es $f_{\text {rev }}=11 \mathrm{kHz}$, lo que permite cruce de haces en los cuatro puntos de interacción (bunch-crossing) con una frecuencia de $\sim 40 \mathrm{MHz}$. Dos de los principales parámetros que definen el potencial de un acelerador de partículas son la energía del centro de masa, definida anteriormente, y la luminosidad instantánea $(\mathcal{L})$, la cual se define como el número de partículas por 
unidad de tiempo y unidad de área, y para colisiones protón-protón, es:

$$
\mathcal{L}=f_{\text {rev }} n_{b} \frac{N_{1} N_{2} \gamma F}{4 \pi \epsilon_{n} \beta^{*}}
$$

donde $N_{i}$ es el número de partículas en cada bunch, $\epsilon_{n}$ es la emitancia transversal normalizada (la dispersión transversal media de las partículas del haz en el espacio de coordenadas e impulsos), $\beta^{*}$ es la función de amplitud en el punto de interacción, relacionada al poder de focalización de los cuadrupolos, $\gamma$ es el factor relativista de Lorentz y $F$ es un factor de reducción geométrico, debido al ángulo de cruce de los haces en el punto de interacción. La luminosidad puede ser entendida como la magnitud que indica la capacidad de un acelerador de producir un determinado número de interacciones de un dado proceso en el que se esté interesado. La tasa de eventos $\left(\frac{d N}{d t}\right)$ de un dado proceso con sección eficaz $\sigma$, puede escribirse como el producto de su sección eficaz y la luminosidad instantánea. Por lo tanto, integrando en el tiempo, se obtiene el número total de eventos $(\mathrm{N})$ a medirse en un dado tiempo t, y se define la luminosidad integrada como:

$$
L=\frac{N}{\sigma}=\int_{0}^{t} \mathcal{L}\left(t^{\prime}\right) \sigma d t^{\prime}
$$

magnitud que suele usarse para describir el tamaño de la muestra colectada. Al final de este capítulo se muestran los valores obtenidos para el período de tiempo analizado en esta tesis, conocido como Run-2. Antes de seguir con la descripción del detector, es oportuno mostrar cómo han sido los períodos de funcionamiento y cuáles son los planes a futuro del LHC para ubicar temporalmente el trabajo llevado a cabo en esta tesis. Un esquema de esto se muestra en la Figura 2.2. El LHC funcionó desde 2010-2012 (período conocido como Run-1), para luego dejar de estar operativo entre 2013 y 2014 (long shutdown 1), donde se le hicieron arreglos y mejoras. En el 2015 se restauraron las colisiones que duraron hasta fines de 2018 (Run-2), alcanzando una energía por haz de $6.5 \mathrm{TeV}(13 \mathrm{TeV})$ y una luminosidad de $1.4 \times 10^{34} \mathrm{~cm}^{-2} \mathrm{~s}^{-1}$. En la actualidad se encuentra en otro período no operativo (long shutdown 2), donde nuevamente se le están haciendo mejoras a los detectores. Se espera que las colisiones comiencen en el 2021 con el inicio del Run-3, que durará hasta el 2024, para luego ingresar en un período más prolongado de inactividad, donde se implementarán mejoras drásticas, que desembocarán en el llamado HLLHC (High Luminosity LHC), que operará hasta 2037 colectando más de $3 a b^{-1}$ de datos.

\subsection{El detector ATLAS}

ATLAS ( $\boldsymbol{A}$ Toroidal $\boldsymbol{L H C} \boldsymbol{A}$ pparatuS ) [56, 57, 58] es uno de los experimentos multipropósito del LHC, diseñado para estudiar las partículas producidas en colisiones protón-protón a las altas energías provistas por el LHC.

Está compuesto por un detector de trazas en la región más interna alrededor del punto de interacción, rodeado por calorímetros y cámaras de muones. Un esquema del detector se puede observar en la Figura 2.3, donde se aprecia su simetría aproximadamente cilíndrica, y se señalan los distintos subdetectores que lo componen. 


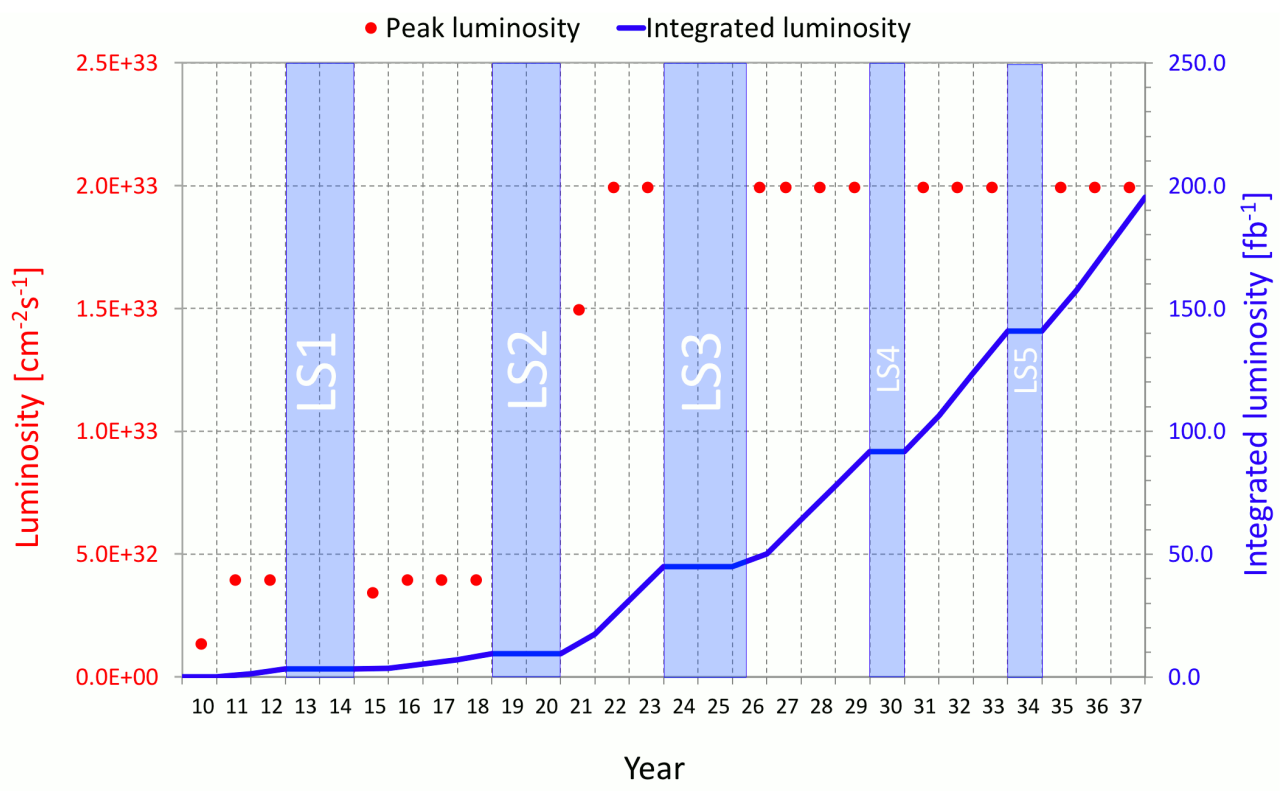

Figura 2.2: Períodos de funcionamiento del LHC desde 2010 hasta 2037.

Se divide geométricamente en dos regiones, la parte central denominada barrel y la región extrema endcap. En la región barrel los detectores se ubican en forma de cilindros concéntricos alrededor del eje del haz, mientras que en la región endcap se disponen como discos perpendiculares a la dirección del haz.

El detector interno de trazas (ID), es el subdetector más próximo al haz, y se encuentra inmerso en un campo magnético de $2 \mathrm{~T}$ (producido por un solenoide superconductor que rodea al ID), necesario para curvar la trayectoria de las partículas cargadas y así medir su impulso. El ID provee una precisa reconstrucción de las trazas de las partículas cargadas, con una alta granularidad. Está a su vez compuesto en primer lugar, empezando del haz hacia afuera, de un detector de pixeles con cuatro capas, cuya primera capa, Insertable B-Layer (IBL), se instaló previo al Run-2 para mejorar el rendimiento, en particular en la determinación de vértices y su consecuente impacto en los estudios que involucran quarks b. Luego cuenta con un detector semiconductor de microbandas de silicio, conocido como SCT, para terminar en la región más alejada del haz, con un detector de radiación de transición (TRT), que brinda la capacidad de identificar y discriminar electrones de hadrones, vía la detección de fotones radiados.

El sistema de calorímetros de dos componentes, se encuentra inmediatamente después del ID. Está diseñado para proveer una cobertura total en $\phi$ y cubrir el rango de pseudorapidez hasta $|\eta|<4$.9. El primero de ellos es el calorímetro electromagnético (ECAL), que mide principalmente la energía depositada por fotones y electrones, mientras que el calorímetro hadrónico (HCAL) mide la energía de los jets y hadrones, y se encuentra rodeando al primero.

Finalmente, la capa más externa de ATLAS consiste de un espectrómetro de muones (MS), donde se intercalan tres imanes toroidales compuestos por ocho bobinas cada uno. Este sistema de imanes provee campos que varían entre los 2 y 6 Tm, y es esencial para curvar la trayectoria de los muones en esta lejana parte del detector. 


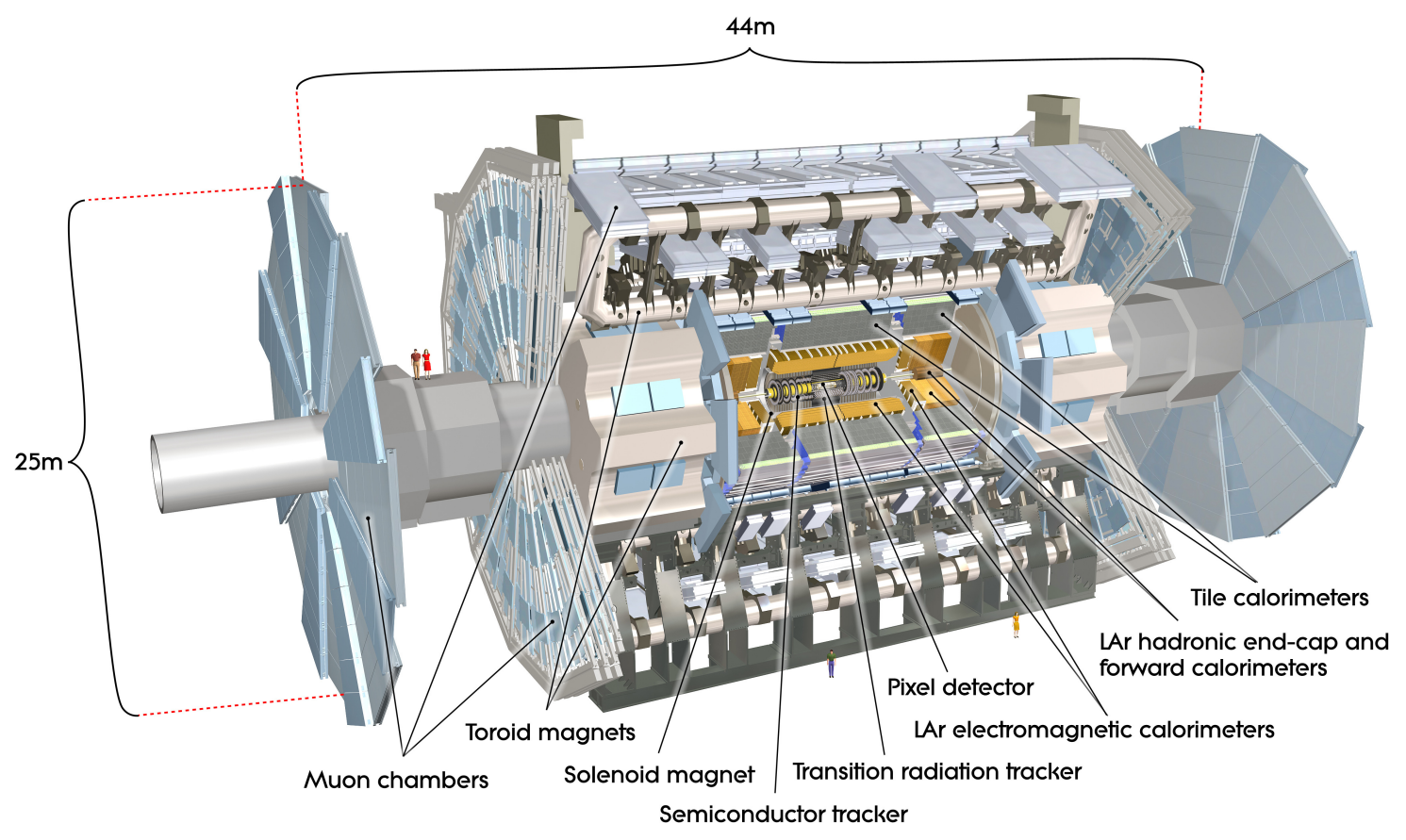

Figura 2.3: Esquema general del detector de ATLAS.

Las dimensiones resultantes de esta geometría y composición, transforman a ATLAS en el detector de partículas más grande construido (hasta el momento) con $46 \mathrm{~m}$ de largo, $25 \mathrm{~m}$ de ancho y del orden de 7000 toneladas de peso.

La Figura 2.4 muestra un esquema del corte transversal de ATLAS, ilustrando los distintos subdetectores y sus funciones en la identificación de las partículas producidas durante las colisiones.

\subsubsection{Sistema de coordenadas}

ATLAS utiliza un sistema de coordenadas cartesiano con su origen en el punto de interacción (IP) nominal, en el centro del detector y con el eje $z$ a lo largo del haz. El eje $x$ apunta desde el IP hacia el centro del anillo del LHC, mientras que el eje $y$ apunta hacia arriba. En el plano transversal se usa un sistema de coordenadas cilíndricas $(r, \phi)$, siendo $\phi$ el ángulo azimutal alrededor del eje del haz. Por último, el ángulo polar $\theta$ se mide con respecto al eje del haz.

En la Figura 2.5 se muestra una representación gráfica del sistema de ejes utilizado para el detector ATLAS y el área experimental, extraído del reporte de diseño técnico de 1999 que puede consultarse en [59].

Se define la rapidez $(y)$ a partir de la energía total de la partícula (E) y la componente longitudinal de su impulso $\left(p_{z}\right)$ según:

$$
y=\frac{1}{2} \ln \left(\frac{E+p_{z}}{E-p_{z}}\right)
$$

donde, a diferencia de la definición usual que se da en relatividad especial, aquí se usa la componente del momento a lo largo del eje del haz, en vez de $|\vec{p}|$. En el límite 


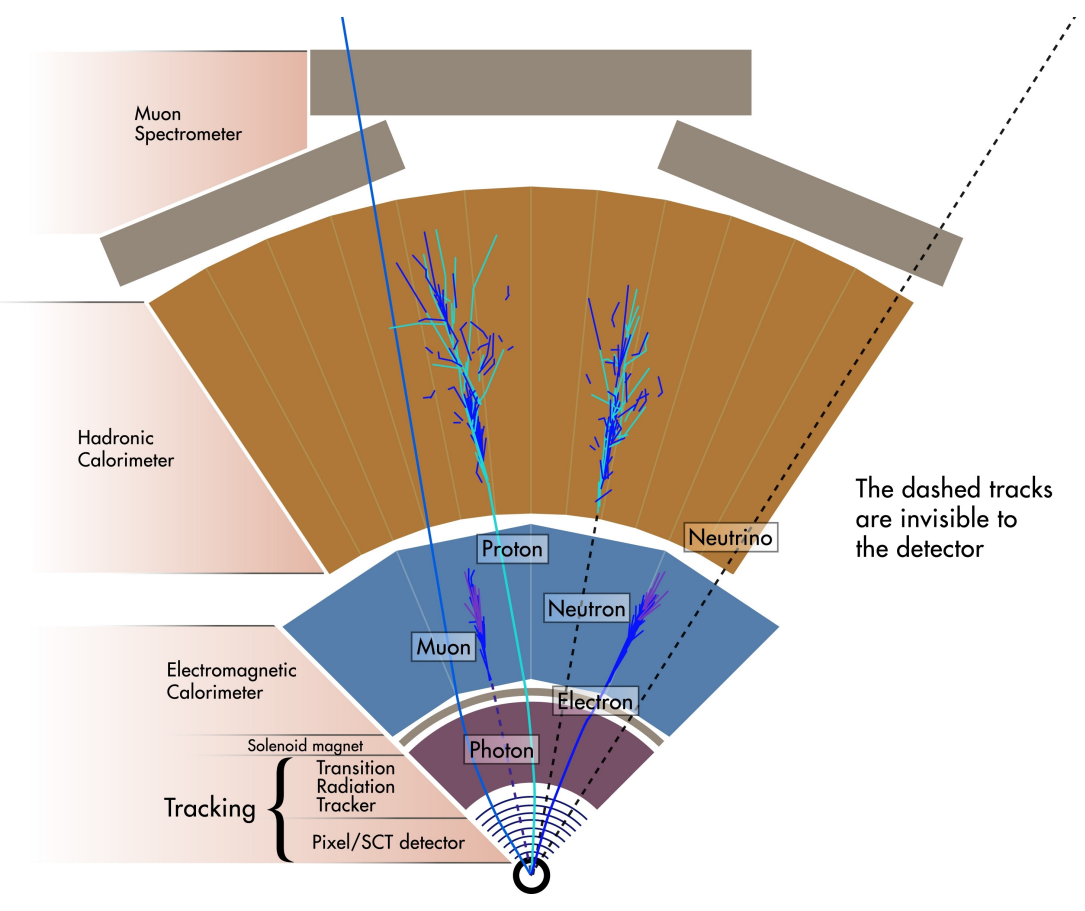

Figura 2.4: Esquema del corte transversal del detector de ATLAS, ilustrando los distintos subdetectores y el pasaje correspondiente de los distintos tipos de partículas.

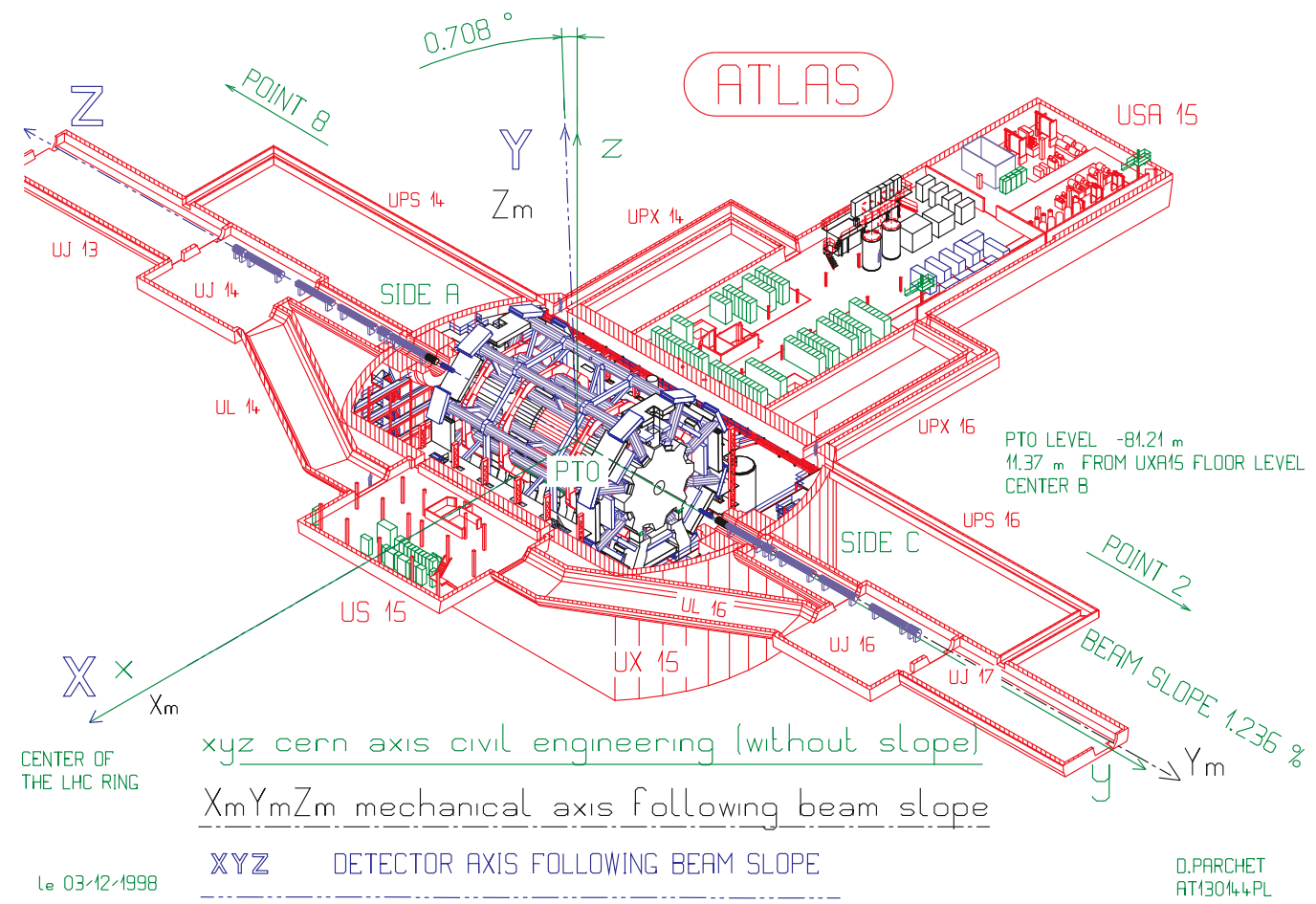

Figura 2.5: Representación gráfica del sistema de ejes utilizado para el detector ATLAS y el área experimental. Reporte de diseño técnico de 1999. 


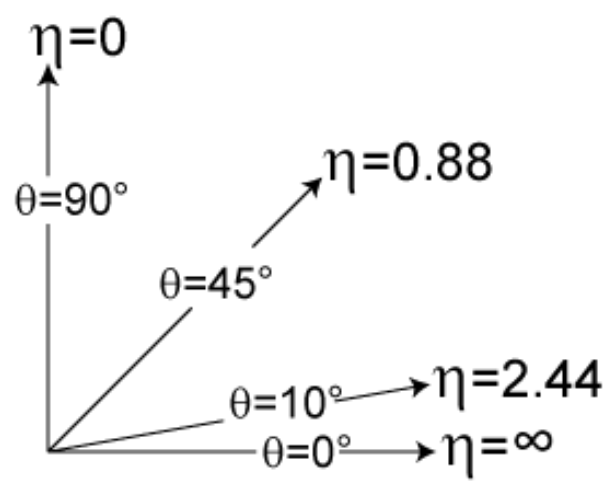

Figura 2.6: Representación gráfica de la relación entre el ángulo polar $\theta$ y la pseudorapidez $\eta$.

de altas energías esta cantidad se aproxima (y vale de forma exacta para partículas no masivas como los fotones) a la pseudorapidez $\eta$, que es una coordenada espacial que describe el ángulo de la partícula con respecto al eje del haz, y se relaciona con el ángulo polar $\theta$ según:

$$
\eta=-\ln \left[\tan \left(\frac{\theta}{2}\right)\right]
$$

Esta nueva variable queda entonces bien definida, e independiente de la masa en el sistema de laboratorio. La razón de preferir a la pseudorapidez sobre el ángulo polar, y realizar esta transformación de coordenadas, se debe al hecho que la multiplicidad de partículas producidas es aproximadamente constante como función de $\eta$, y que la diferencia de pseudorapidez entre dos partículas es invariante frente a transformaciones de Lorentz (boosts) a lo largo de la dirección del haz. Una comparación gráfica entre $\eta$ y $\theta$ se muestra en la Figura 2.6, donde puede verse, por ejemplo, que la pseudorapidez crece para ángulos pequeños, lo que suele denominarse como la región forward.

Ya que el ángulo $\phi$ es también invariante de Lorentz, se puede definir la distancia angular entre partículas $\Delta R$ como:

$$
\Delta R \equiv \sqrt{(\Delta \eta)^{2}+(\Delta \phi)^{2}}
$$

Por último es importante mencionar la razón por la cual sólo las componentes transversales se tienen en cuenta para los análisis en colisionadores hadrónicos, como por ejemplo $E_{T}(=E \sin \theta)$ o $p_{T}(=p \sin \theta)$. Los protones inciden en la dirección $z$, por lo que si se supone que el momento transverso de los partones es nulo, el momento transversal total se conserva durante la colisión. Se puede entonces definir la energía transversa de una partícula, en función de la pseudorapidez como:

$$
E_{T}=\frac{E}{\cosh \eta}
$$

donde $E$ es su energía total. La aplicación de las leyes de conservación no es posible en la dirección longitudinal, debido a que la fracción original de impulso del protón incidente de cada uno de los partones interactuantes es desconocida. 


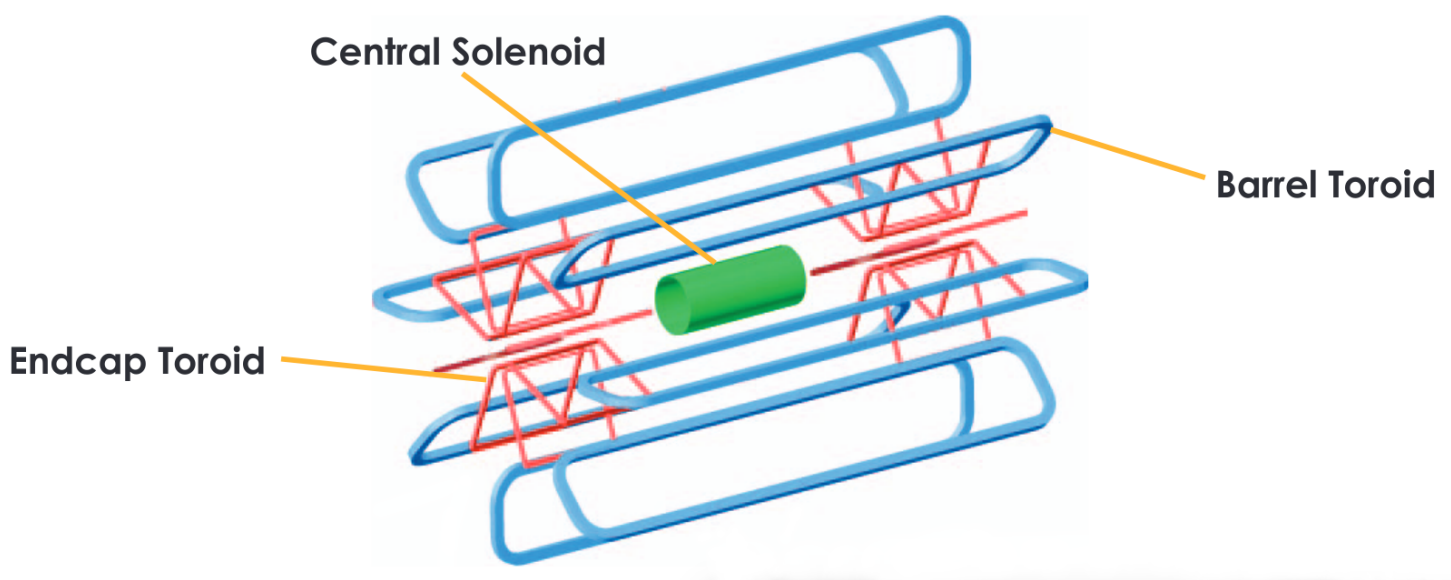

Figura 2.7: Esquema del sistema de imanes de ATLAS.

\subsubsection{Sistema de imanes}

El sistema de imanes de ATLAS [60] es de vital importancia ya que permite una precisa medición del momento de todas las partículas cargadas producidas, además de la identificación de la propia carga. Está compuesto por tres conjuntos de imanes superconductores como se muestra en la Figura 2.7.

El primero de los imanes superconductores es el solenoide central, paralelo al eje del haz, que rodea al detector interno y provee un campo magnético de $\sim 2 \mathrm{~T}$ que sirve para reconstruir el momento transverso de las partículas cargadas. Luego se encuentran los dos toroides especializados para el espectrómetro de muones. En la región barrel, se dispuso un masivo toroide de $26 \mathrm{~m}$ de largo y $20 \mathrm{~m}$ de diámetro, compuesto por ocho bobinas, que provee una intensidad de campo magnético medio de $\sim 0.5$ T. Finalmente, otros dos toroides completan este sistema, ubicados en las regiones endcap, formados también por ocho bobinas, y que proveen al MS con $1 \mathrm{~T}$. La refrigeración, que mantiene su condición superconductora, se hace mediante un sistema de circulación de helio líquido a $4.5 \mathrm{~K}$.

\subsubsection{Detector interno}

El detector interno (ID), cuyo esquema general se muestra en la Figura 2.8, es el más próximo al haz y combina detectores de muy alta resolución (cerca del punto de interacción) con detectores continuos de trazas, y está contenido dentro del solenoide central del sistema de imanes. Los distintos detectores que lo componen se describen a continuación, y se puede ver un corte transversal de los mismos en la Figura 2.9. Su resolución (de diseño) de momento transversal inverso viene dada por la Ecuación 2.6 ,

$$
\frac{\sigma\left(p_{\mathrm{T}}\right)}{p_{\mathrm{T}}}=0.05 \% \cdot p_{\mathrm{T}} \oplus 1 \%
$$

donde $\oplus$ significa una suma en cuadratura, y la resolución en la posición de los vértices puede llegar a valores de aproximadamente $20 \mu \mathrm{m} / 20 \mu \mathrm{m} / 50 \mu \mathrm{m}(\mathrm{x} / \mathrm{y} / \mathrm{z})[61]$. 


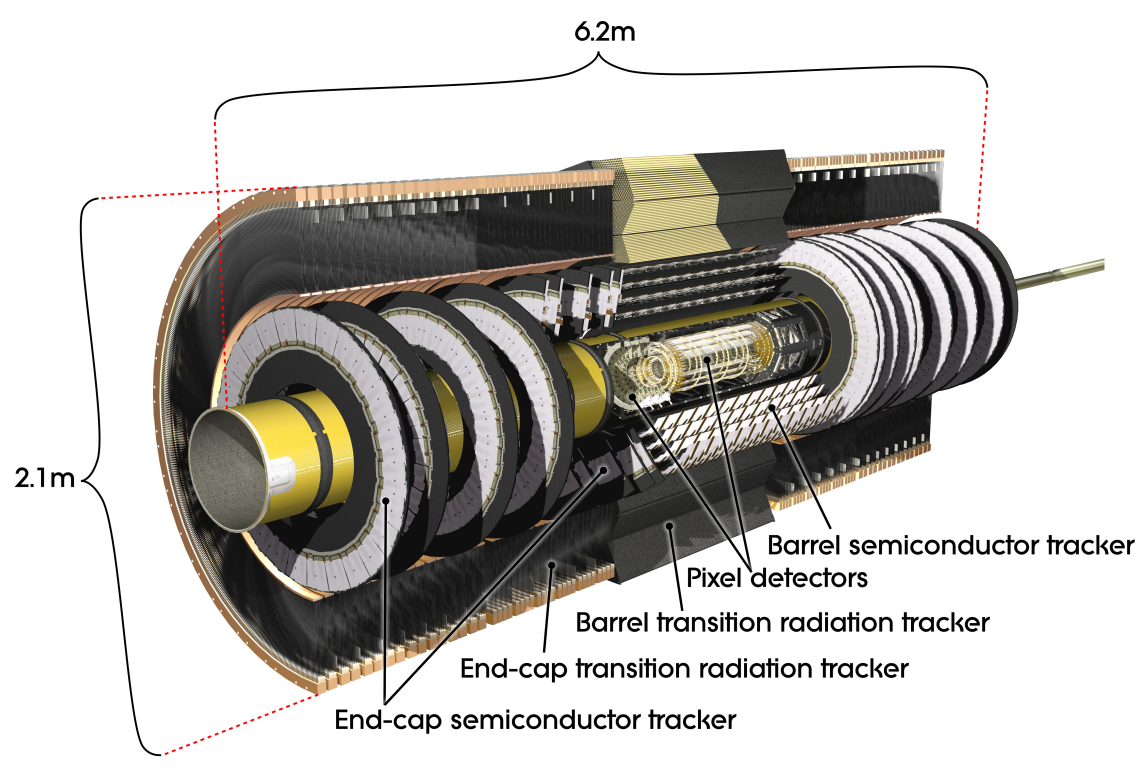

Figura 2.8: Esquema general del detector interno de ATLAS.

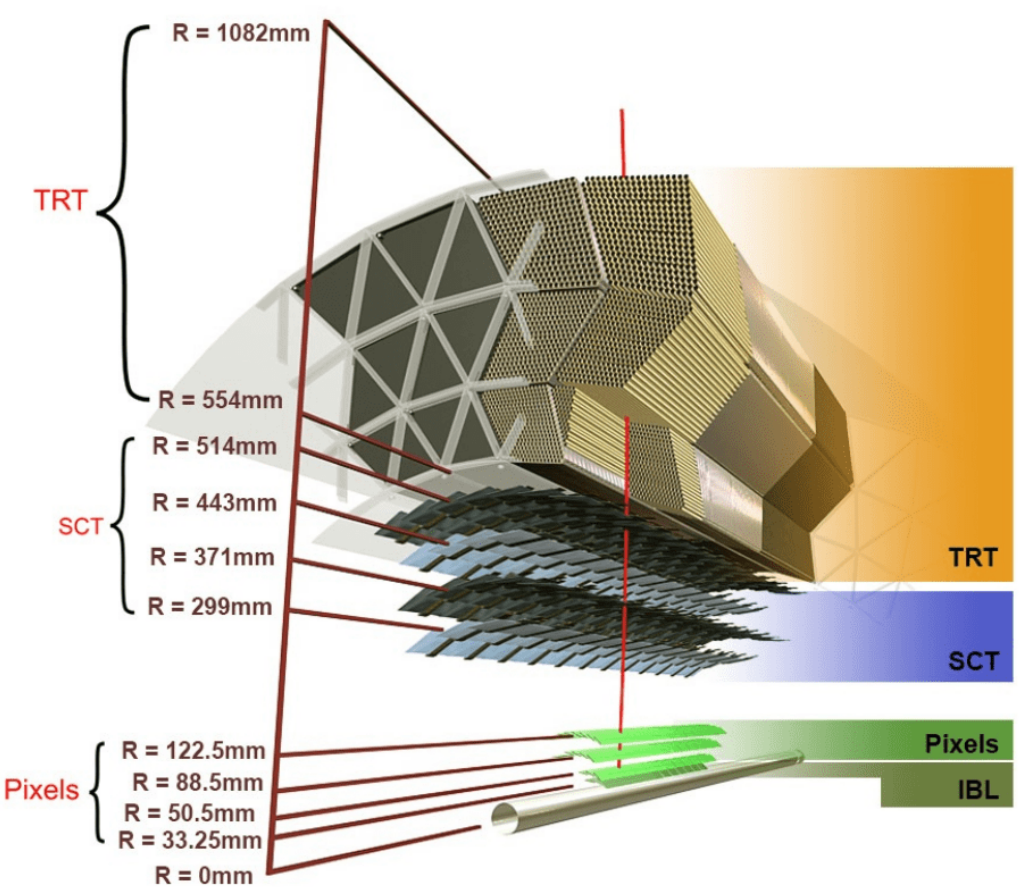

Figura 2.9: Esquema del detector interno mostrando la traza de una partícula cargada de $p_{T}=10 \mathrm{GeV}$ atravesándolo. La trayectoria atraviesa el tubo del haz de berilio, las tres capas del detector de píxeles de silicio, las cuatro capas dobles del SCT, y aproximadamente 36 tubos contenidos en los módulos del TRT. 


\section{Detector de Píxeles}

El detector de píxeles fue construido para medir la posición de las trazas de partículas cargadas con la más alta precisión posible y es de vital importancia para la reconstrucción de los vértices primarios y secundarios. Se compone de cuatro capas, cuya primera y más cercana al haz, la Insertable B-Layer o IBL, se agregó durante el Long-Shutdown 1, debido a la alta luminosidad esperada para el Run-2, y el daño que podría causar en el resto de las capas de píxeles, además de proveer una mejora en la eficiencia de identificación de trazas, vértices, y en la identificación de b-jets, que decaen típicamente fuera del radio del IBL. El IBL se encuentra a tan solo $3.3 \mathrm{~cm}$ del punto de interacción, está compuesto por ocho millones de chips de rápida lectura y con sensores de silicio, que detectan el paso de partículas cargadas mediante la deposición de carga inducida. El tamaño de los píxeles es de $50 \times 250 \mu \mathrm{m}^{2}$, con una resolución de $8 \mu \mathrm{m}(R-\phi)$ y $40 \mu \mathrm{m}(z)$.

El resto del detector de píxeles se compone, en la región barrel, de tres capas cilíndricas, mientras que la endcap de tres discos perpendiculares al eje del haz. La capa más interna, quien fuera durante Run-1 la B-Layer, se encuentra rodeando al IBL y a $17 \mathrm{~mm}$ del mismo. El principio de detección para partículas cargadas es la medida de la deposición de la carga inducida en una capa de silicio por ionización. El sistema contiene un total de 80 millones de sensores, cada uno con una resolución de $10 \mu \mathrm{m}(R-\phi)$ y $115 \mu \mathrm{m}(z)$. Las tres capas de la región barrel cubren la región $|\eta|<1.9$, mientras que los discos de la región endcap extienden la cobertura hasta $|\eta|<2.5$.

\section{Detector Semiconductor de Trazas (SCT)}

Rodeando al detector de píxeles, se encuentran las cuatro capas de módulos de sensores del SCT en la región barrel, y los nueve discos transversales al eje del haz en el endcap. El SCT está diseñado para medir las trazas con alta precisión en la zona intermedia del detector, cubriendo en el rango $|\eta|<2.5$. Está formado por sensores de silicio segmentados en micro bandas, dada la más baja multiplicidad de partículas esperada, lo que permite una reducción de costos y de canales de read-out necesarios. La resolución espacial es de $17 \mu \mathrm{m}(R-\phi)$ y $580 \mu \mathrm{m}(z)$.

\section{Detector de Radiación de Transición (TRT)}

En la parte más externa del ID se encuentra el TRT, diseñado no solo para detectar partículas cargadas, sino también para distinguir entre partículas pesadas y livianas. El TRT se compone de tubos de deriva, de $4 \mathrm{~mm}$ de diámetro, que contienen un gas mezcla de $70 \% \mathrm{Xe}, 27 \% \mathrm{CO}_{2}$ y $3 \% \mathrm{O}_{2}$, que se ioniza al ser atravesado por partículas cargadas, y se detecta la radiación de transición. Los electrones producidos son colectados por una ánodo, y el tiempo de deriva es una medida de la distancia hasta la traza del mismo con una resolución de $130 \mu \mathrm{m}$. Además, los tubos están cubiertos con fibras de polipropileno con un índice de refracción diferente, por lo que las partículas que atraviesan el detector emiten radiación con una intensidad proporcional a $\gamma=E / m$. De esta forma, el TRT permite distinguir partículas cargadas pesadas $\left(\pi^{ \pm}\right)$de aquellas más livianas $\left(e^{ \pm}\right)$. La región barrel contiene 50000 tubos paralelos al eje del haz y la región endcap 320000 tubos orientados radialmente. Su resolución espacial es de $0.17 \mathrm{~mm}$, y su cobertura abarca el rango $|\eta|<2.0$. 


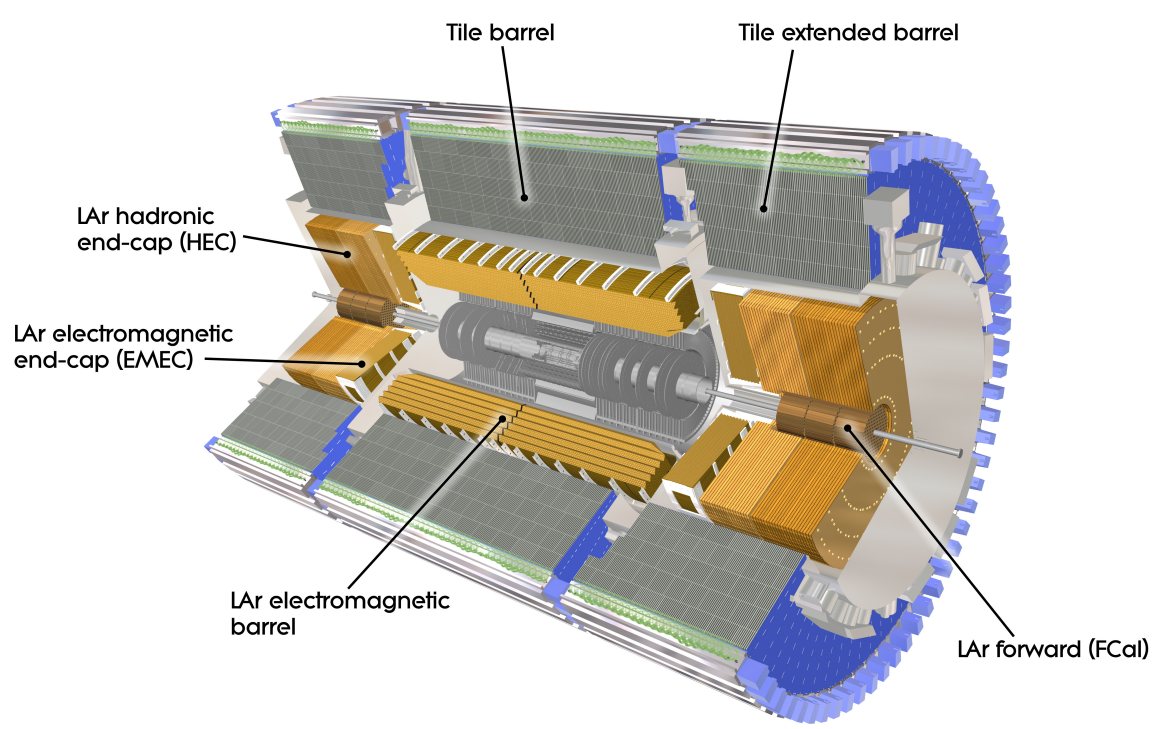

Figura 2.10: Sistema de calorímetros del detector ATLAS.

\subsubsection{Calorímetros}

El sistema de calorímetros de ATLAS está formado por una componente electromagnética (ECAL) y una hadrónica (HCAL), y está diseñado para medir la energía y la posición de las partículas incidentes, mediante la absorción de la energía depositada por las cascadas de partículas secundarias generadas en el material por las partículas producidas en la colisión. Provee una cobertura completa en $\phi$ y el rango en la pseudorapidez es $|\eta|<4.9$, con una granularidad más fina en la región que coincide con el ID. Este sistema, haciendo uso de sus dos componentes, permite discriminar entre electrones y fotones de los hadrones (jets) producidos. Permite además, medir el desbalance energético (gracias a su casi total cobertura y hermeticidad) y provee la información utilizada por el sistema de trigger (que se detalla más adelante) para la selección online de eventos potencialmente interesantes. Este sistema se muestra en la Figura 2.10. Por su parte, la resolución (de diseño) en la energía calorimétrica viene dada por la Ecuación 2.7,

$$
\frac{\sigma(E)}{E}=\frac{a}{\sqrt{E}} \oplus b \oplus \frac{c}{E}
$$

donde $\oplus$ significa una suma en cuadratura. El término estocástico $\frac{a}{\sqrt{E}}$ está relacionado con las fluctuaciones en el desarrollo de las lluvias, el término constante $b$ tiene en cuenta las inhomogeneidades en el detector, y el último término está asociado con el ruido electrónico y es proporcional al $\frac{1}{E}$. El valor de los coeficientes $a$ y $b$ dependen de los objetos incidentes. Para el caso de electrones en el ECAL se tiene que $a \sim 10 \% \mathrm{GeV}^{1 / 2}$ y $b \sim 0.7 \%$, mientras que para piones cargados en el centro del detector se tiene $a \sim 50 \% \mathrm{GeV}^{1 / 2}$ y $b \sim 5 \%$ [62].

\section{Calorímetro Electromagnético (ECAL)}

El ECAL es un calorímetro de muestreo inhomogéneo no compensado, con una geometría de acordeón, que utiliza plomo $(\mathrm{Pb})$ como material absorbente y argón 
líquido (LAr) como medio ionizable. Está dividido en dos mitades dentro de la región barrel $(-1.475<\eta<0$ y $0<\eta<1.475)$ y en dos componentes (una a cada lado) en los endcap $(1.375<|\eta|<3.2)$. La región de transición entre el barrel y el endcap $(1.37<|\eta|<1.52)$, es una zona no instrumentada por donde se conecta el detector [56]. Esta región se denomina crack, y la mayoría de los análisis requiere que los candidatos a fotones/electrones estén fuera de ella.

Las partículas incidentes interactúan con las placas de $\mathrm{Pb}$, creando una lluvia de partículas cargadas y neutras. Las partículas cargadas ionizan el LAr que se encuentra entre las placas, y un electrodo central de kapton/Cu colecta los electrones producidos, vía un campo eléctrico aplicado. La señal total en el medio activo es así proporcional a la energía total real de la partícula incidente.

En la región diseñada para medidas de precisión $(|\eta|<2.5$, excluyendo el crack), el ECAL está segmentado en tres capas longitudinales. La primera capa consiste de bandas con fina granularidad (en la dirección de $\eta$ ), para discriminar entre fotones aislados y pares de fotones espacialmente cercanos provenientes del decaimiento $\pi^{0} \rightarrow \gamma \gamma$. Para los electrones y fotones con alta energía transversa, la mayoría de la energía se colecta en la segunda capa, que tiene una granularidad lateral de $0.025 \times 0.025$ en $(\eta, \phi)$. La tercer capa se encarga de la energía depositada en las colas de la lluvia.

El espesor del ECAL es mayor a 22 longitudes de radiación $\left(X_{0}\right)$ en la región barrel, y mayor a $24 X_{0}$ en los endcap, donde una longitud de radiación se define como la distancia promedio sobre la cual la energía de un electrón se reduce a $1 / e$ de su energía inicial. Para el caso de los fotones, una reducción similar se obtiene a $9 / 7$ de $X_{0}$. Por tanto, toda la energía electromagnética es absorbida en el ECAL y sólo parte de la componente hadrónica llega al HCAL.

\section{Calorímetro Hadrónico (HCAL)}

Tres capas de calorímetro hadrónico rodean al ECAL, y brindan una discriminación adicional para electrones y fotones al medir la energía hadrónica. El HCAL se extiende en pseudorapidez hasta $|\eta|<4.9$, permitiendo cubrir prácticamente la totalidad de los $4 \pi$ desde el punto de interacción. En la región barrel $(|\eta|<1.7)$, se ubica el Tile Calorimeter, un calorímetro de muestreo que utiliza acero como material absorbente y tejas centelladoras plásticas como material activo. A su vez está dividido en dos partes $(|\eta|<1.0$ y $0.8<|\eta|<1.7)$. Los centelladores, dispuesto en un arreglo periódico, se conectan a una fibra óptica que trasporta la luz producida por el paso de partículas, hacia un tubo fotomultiplicador. Este arreglo se extiende, en R, de los 2.28 a los $4.25 \mathrm{~m}$. En la región endcap $(1.5<|\eta|<3.2)$ se encuentra un calorímetro hadrónico de muestreo (HEC) con placas de cobre como absorbente y argón líquido como material activo. Cada lado del endcap consiste en dos ruedas, una atrás de la otra con las placas planas de $\mathrm{Cu}$ dispuestas perpendicularmente al eje del haz, con un radio de $2.3 \mathrm{~m}$. Finalmente se encuentra el Forward Calorimeter (FCAL), un calorímetro de muestreo que extiende la cobertura del sistema a $|\eta|<4.9$, coaxial al eje del haz y ubicado a $4.7 \mathrm{~m}$ a cada lado del punto de interacción. El material principal de los módulos es argón líquido (con cobre o tungsteno), y si bien no se utiliza para mediciones de precisión, provee información para el cómputo de la energía transversa faltante y la reconstrucción de jets en regiones muy cercanas al eje del haz. 
Por su parte, el HCAL tiene un espesor mayor a 7.7 longitudes de interacción hadrónica $(\lambda)$ en la región barrel (9.7 $\lambda$ en total si se cuenta el ECAL). De manera análoga a la longitud de radiación mencionada para el ECAL, una longitud de interacción hadrónica se define como la distancia promedio sobre la cual la energía de un hadrón se reduce a $1 / e$ de su energía inicial. De esta forma, toda la energía con la que llegan los hadrones al HCAL, queda allí depositada.

\subsubsection{Espectrómetro de Muones}

El espectrómetro de muones (MS) es la última pieza del detector y el responsable del enorme tamaño final que posee ATLAS. Los muones de alto $p_{T}$ generados en el punto de interacción son poco interactuantes y son las únicas partículas (detectables) capaces de alcanzar el MS. Intercalado con este, se encuentran los toroides del sistema de imanes, y el MS está diseñado para obtener mediciones de alta precisión de la posición e impulso de los muones, junto con una rápida identificación para el sistema de trigger.

El MS posee diferentes tipos de cámaras de detección, como se muestra en la Figura 2.11. Las cámaras para medidas de precisión, denominadas Monitored Drift Tubes (MDT) cubren el rango de $|\eta|<2.7$, y funcionan de forma similar al TRT, con tubos llenos de un gas que se ioniza y un ánodo central que recoge los electrones producidos, y el tiempo de deriva se asocia con la distancia a la traza. En la región endcap, con $|\eta|>2.0$, frente a los dos toroides laterales, se encuentran las Cathode Strip Chambers (CSC) que poseen alta resolución espacio-temporal. Estas cámaras funcionan midiendo la carga depositada en un ánodo, producto de la cascada de electrones creados cerca del mismo. Por último, el MS posee dos tipos más de cámaras, orientadas a brindar información al sistema de trigger, con una resolución espacial menor que las anteriores, pero con una alta velocidad para el primer nivel del trigger. En la región barrel, con $|\eta|<1.05$, las Resistive Plate Chamber (RPC) proveen una estimación rápida del momento de los muones. Las RPCs miden la descarga ocasionada entre dos placas resistivas paralelas sometidas a una alta diferencia de potencial, tras la ionización del volumen de gas interno causada por el paso de muones energéticos. En la región endcap, con $|\eta|<2.4$, se encuentran las Thin Gap Chambers (TGC), similares en funcionamiento a las CSC.

\section{3. $\quad$ El sistema de trigger}

El sistema de trigger de ATLAS [63, 64] utiliza información del detector para rechazar eventos que no posean una física interesante (física ya conocida por ejemplo), reduciendo la frecuencia de eventos de $40 \mathrm{MHz}$ (frecuencia de bunch-crossing mencionada en la Sección 2.1) a alrededor de $1.5 \mathrm{kHz}$. Es necesario destacar aquí el rol central que cumple el sistema de trigger para el buen funcionamiento de todo el experimento, siendo el responsable de decidir qué eventos se guardan y, en definitiva, qué física se encontrará (o no) durante el análisis de los mismos. Sin un sistema de trigger eficiente, todos los subdetectores antes descriptos serían desaprovechados.

Para lograr tal reducción en la frecuencia de eventos y, al mismo tiempo, tener una alta eficiencia en la selección de aquellos de interés, el sistema de trigger está compuesto por dos niveles consecutivos capaces de realizar una identificación de partículas cada vez más compleja; un primer nivel de trigger (L1) basado en hardware 


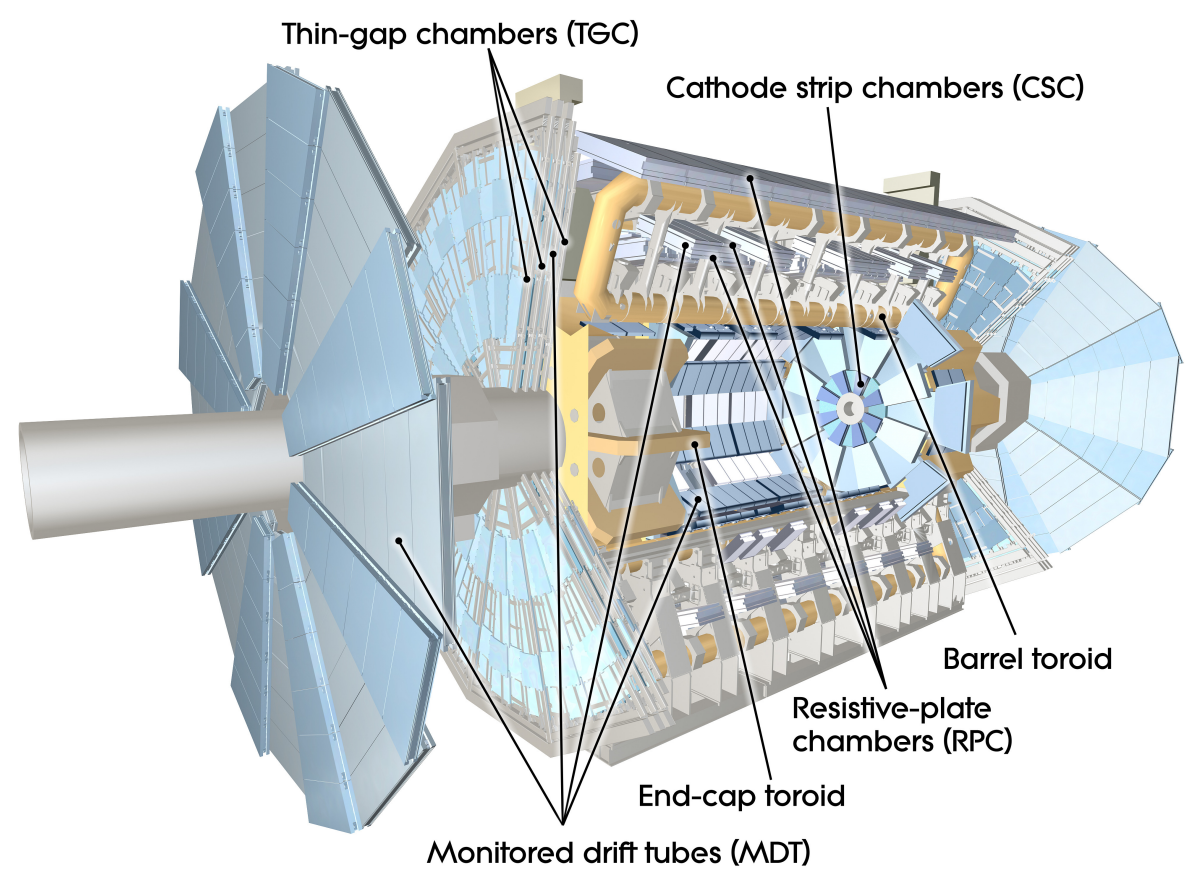

Figura 2.11: Espectrómetro de muones del detector ATLAS.

y luego un trigger basado en software de alto nivel (HLT). Cada nivel permite analizar los eventos con mayor detalle, aumentando la precisión de los criterios de selección y la complejidad de los algoritmos utilizados. El sistema de adquisición de datos (DAQ) transfiere y almacena los datos seleccionados por el trigger. La Figura 2.12 muestra un esquema del sistema de Trigger-DAQ (TDAQ) de ATLAS [64]. A continuación se detallan las dos componentes del trigger y la información que utilizan para la selección de los eventos.

\subsubsection{L1: Trigger basado en Hardware}

La decisión del trigger comienza con el Level-1, basado en hardware [65], que identifica las Regiones de Interés (RoIs). Estas RoIs se definen a partir de la posición en el calorímetro de cada objeto encontrado en un evento potencialmente interesante, que se extiende como un cono desde el punto de interacción a lo largo del detector. Lo mismo sucede en el detector de muones, que tiene diferentes cámaras (descriptas en la Sección 2.2.5) que permiten obtener una estimación rápida del $p_{T}$ de los muones. El diseño del L1 permite tener una aceptancia en el rango de $|\eta|<2.5$ para electrones, fotones, muones y taus, hasta $|\eta|<3.2$ para jets, y $|\eta|<4.9$ para el cálculo del momento transverso faltante.

El L1 utiliza señales de granularidad reducida de los calorímetros y de las cámaras de muones para definir las RoIs con clusters de alta $E_{\mathrm{T}}$ en los calorímetros o trazas de muones en las cámaras de muones. Con esto, debe tomar la decisión que reduce la taza de eventos de $40 \mathrm{MHz}$ a menos de $100 \mathrm{kHz}$, en aproximadamente $2.5 \mu \mathrm{s}$, tiempo determinado en parte por el limitado tamaño de los buffers de memoria y en parte por el tiempo que le toma a los muones producidos en el evento alcanzar el MS.

Las RoIs generadas por el L1 sirven luego como semillas para el HLT, que utiliza 


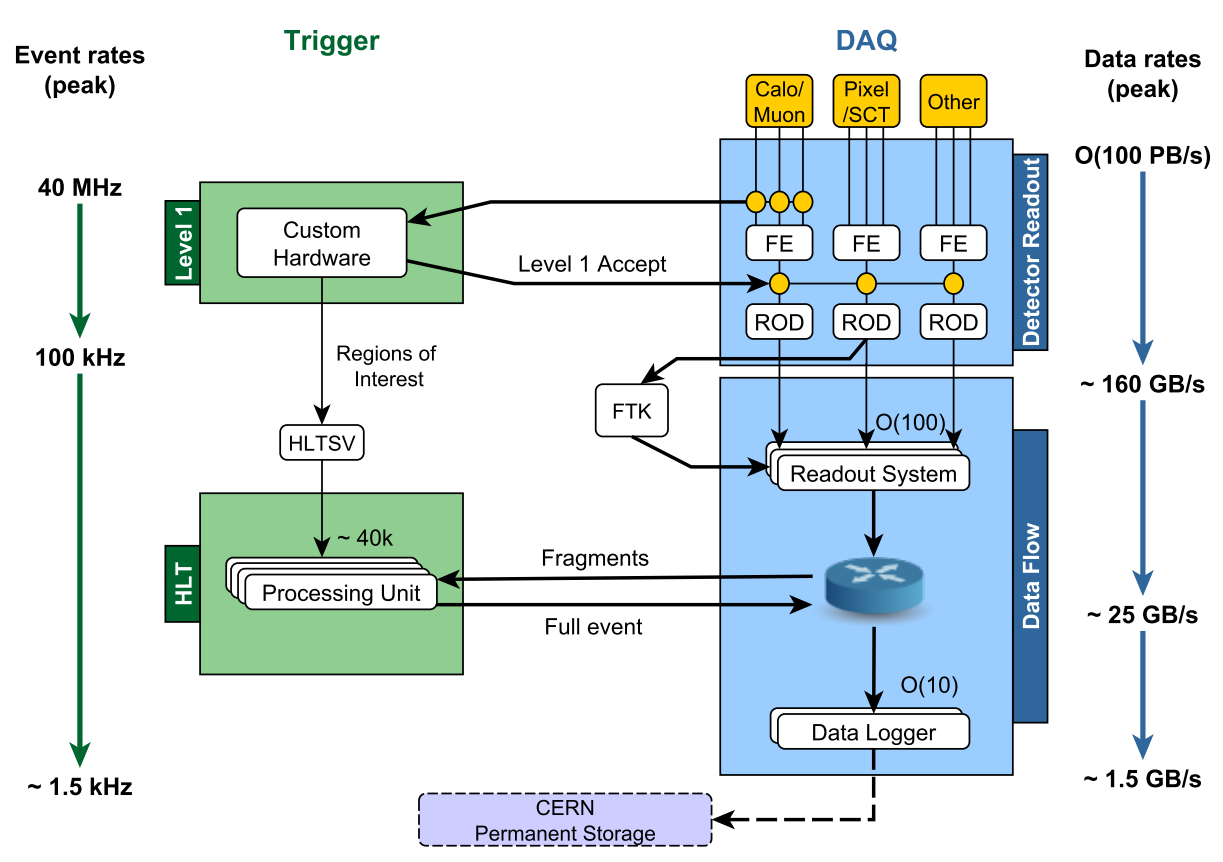

Figura 2.12: Diagrama esquemático del sistema de niveles del trigger y del flujo de datos en ATLAS.

la granularidad completa del detector en la RoI para proporcionar la decisión final del trigger, que determina si el evento se guardará para el análisis offline.

\subsubsection{HLT: Trigger basado en Software}

Cuando un evento es aceptado por el L1, el High Level Trigger [66] ejecuta una secuencia de algoritmos a partir de las RoIs definidas por el L1, y permite reducir la tasa de eventos que se almacena a $1.5 \mathrm{kHz}$ en $0.2 \mathrm{~s}$. La reconstrucción e identificación de las partículas candidatas en el HLT se evalúa en una secuencia de pasos donde se aplican diferentes algoritmos. Si la selección falla en un determinado paso, los siguientes ya no se ejecutan para ahorrar tiempo de ejecución.

En el HLT, los algoritmos se agrupan en conjuntos de algoritmos de reconstrucción rápida ejecutados primero, y después se ejecuta un conjunto de algoritmos de reconstrucción de precisión similar a los que se usan offline, gracias al tiempo de latencia disponible. Los algoritmos de reconstrucción rápida utilizan la información de los calorímetros y de las trazas sólo dentro de la RoI para realizar la selección e identificación de los candidatos, y realizar el rechazo de fondo lo más rápido y temprano posible. Si la partícula candidata pasa los criterios definidos por la selección de reconstrucción rápida, se ejecutan los algoritmos de selección de precisión. Estos tienen acceso a la información del detector fuera de la RoI, con la máxima granularidad e incluyendo detalles sobre la calibración de energía de los calorímetros, la alineación de los subdetectores y el mapa de campo magnético.

Los algoritmos del HLT se ejecutan en aproximadamente 40 mil núcleos de CPU. Además, la construcción parcial de eventos se utiliza para el análisis a nivel del trigger, el monitoreo del detector y las calibraciones de los subsistemas del detector. 
Los eventos aceptados por el HLT son finalmente grabados a disco y distribuidos, accesibles offline para todos los diferentes estudios y análisis.

\subsubsection{Cadenas y menú del trigger}

Una secuencia de algoritmos del L1 y el HLT se denomina Trigger chain y tiene por objeto identificar las características de las partículas que llegan al detector desde la colisión. Se puede definir una cadena para identificar una o más partículas de un tipo dado y con un umbral dado de $E_{\mathrm{T}}$. El conjunto completo de cadenas de trigger que se evalúan simultáneamente online se denomina Trigger Menu, y su composición se optimiza para las condiciones del LHC, de manera de no afectar el buen funcionamiento del sistema de adquisición de datos.

En ATLAS, el nombre de la cadena del trigger codifica información sobre la hipótesis de la multiplicidad de partículas requeridas, el tipo de partícula y el sabor, sus criterios de selección y su mínima $E_{\mathrm{T}}$. Por ejemplo, la cadena llamada 2g20_tight representa una cadena que requiere al menos dos fotones, ambos con al menos 20 $\mathrm{GeV}$ de impulso transverso que pasen un criterio de identificación de fotones tight (los criterios de identificación se definen en el Capítulo 3).

Para mantener las tasas de eventos dentro de los parámetros de diseño, a algunas de las cadenas de trigger se les puede asignar un pre-escaleo (PS), que define la frecuencia con la que esa dada cadena será evaluada por el trigger. Es decir que se evaluará un evento de cada PS eventos. Cuando PS $=1$ se dice que el trigger está unprescaled, siendo evaluado en todos los eventos. Estos PS se mantienen igual a 1 para los triggers principales (primary triggers usados para los análisis de física) y para el resto se modifican dinámicamente durante la toma de datos en base a la luminosidad provista por el LHC, manteniendo la tasa de procesamiento constante, sin consumir todo el ancho de banda. También hay cadenas de triggers configuradas para no aceptar eventos online, sino que los algoritmos se ejecutan una vez que se sabe que el evento es aceptado por otros triggers. Se dice que estos triggers se encuentran configurados en modo rerun, y se utilizan para determinados estudios de la eficiencia, ya que la decisión del trigger está disponible para su uso offline.

\subsection{Manejo y distribución de datos y recursos compu- tacionales}

La arquitectura computacional de ATLAS está diseñada para permitir a todos los miembros de la colaboración un acceso ágil, directo y distribuido a la gran cantidad de datos colectados por el detector ( $\sim \mathrm{PB} / \mathrm{año})$. La arquitectura se basa en la tecnología GRID [67], compartiendo el poder de procesamiento y la capacidad de almacenamiento disponibles en distintos centros de cómputo asociados alrededor del mundo.

El software de ATLAS se desarrolla dentro un entorno $\mathrm{C}++$ común llamado ATHENA [68, 69, 70], en el que se realiza todo el procesamiento de datos. Los eventos aceptados por el trigger deben ser procesados para reducir su tamaño y ser utilizados para los análisis offline.

A la salida del HLT, los eventos son almacenados como Raw Data Objects (RDOs). Luego de aplicar los algoritmos de reconstrucción y calibración, las co- 


\subsection{MANEJO Y DISTRIBUCIÓN DE DATOS Y RECURSOS COMPUTACIONALES43}

lecciones de los distintos objetos físicos obtenidas son almacenadas en formato ESD (Event Summary Data) y AOD (Analysis Object Data), una versión reducida del primero ( $\sim 100 \mathrm{kB} /$ evento). A partir de las ESDs/AODs, se ha definido un formato de datos significativamente más pequeño (10-15 kB/evento) conocido como xAOD, sobre el que se realiza el análisis final. Las xAOD son archivos accesibles vía el entorno de análisis de datos ROOT [71], que contienen el conjunto de variables de diferentes objetos físicos, según las necesidades de los distintos grupos de análisis dentro de ATLAS. En base a esto y para agilizar el análisis final, la colaboración preselecciona eventos offline en las llamadas derivaciones.

Desde los raw data hasta las distintas derivaciones, se aplican distintos criterios de sliming (se remueven los eventos que no son de interés), skiming (se remueve la información irrelevante de los objetos) y thining (se remueven objetos y/o colecciones de objetos irrelevantes) según los estudios y análisis que se vayan a realizar sobre los datos colectados.

En esta tesis se usan principalmente las denominadas EGAM9, SUSY4 y SUSY1, las cuales realizan una preselección de eventos optimizados para distintos estudios como se describe a continuación.

Las derivaciones EGAM9 y SUSY4 se utilizan para los estudios de eficiencia del trigger presentados en el Capítulo 4. La primera se desarrolló específicamente para realizar los estudios del trigger de fotones que se presentan en esta tesis, y está disponible para su uso desde fines de 2015. Los eventos que se almacenan deben cumplir con al menos uno de los requisitos siguientes:

- El evento debe haber sido seleccionado por cualquier cadena de trigger HLT_noalg_* o de un fotón loose HLT_g*_loose*.

- Al menos un electrón o un fotón loose con $p_{\mathrm{T}}>10 \mathrm{GeV}$.

Para las eficiencias del trigger de multijets se utiliza la derivación SUSY4, diseñada para búsquedas de SUSY en eventos con multijets, donde se almacenan aquellos eventos que cumplen con alguno de los siguientes requerimientos:

- El evento debe haber sido seleccionado por un trigger con al menos cinco jets: HLT_nj*, con $n \geq 5$.

- Al menos un leptón loose con $p_{\mathrm{T}}>15 \mathrm{GeV}$ y $|\eta|<2.6$, y al menos tres jets con $p_{\mathrm{T}}>40 \mathrm{GeV}$ y $|\eta|<2.5$.

- Al menos dos leptones loose con $p_{\mathrm{T}}>15 \mathrm{GeV}$ y $|\eta|<2.6$, y al menos dos jets con $p_{\mathrm{T}}>40 \mathrm{GeV}$ y $|\eta|<2.5$.

Por último, la derivación SUSY1 contiene todos los objetos y cantidades necesarias para la búsqueda llevada a cabo en el Capítulo 5, ya que se almacenan los eventos que cumplan con al menos uno de los requisitos siguientes:

- La suma del $p_{\mathrm{T}}$ de todos los jets del evento que tengan $p_{\mathrm{T}}>40 \mathrm{GeV}$ y $|\eta|<2.8$, debe exceder $150 \mathrm{GeV}$.

- Al menos un electrón identificado como loose con $p_{\mathrm{T}}>100 \mathrm{GeV}$ y $|\eta|<2.6$, o dos electrones con $p_{\mathrm{T}}>20 \mathrm{GeV}$ y $|\eta|<2.6$. 


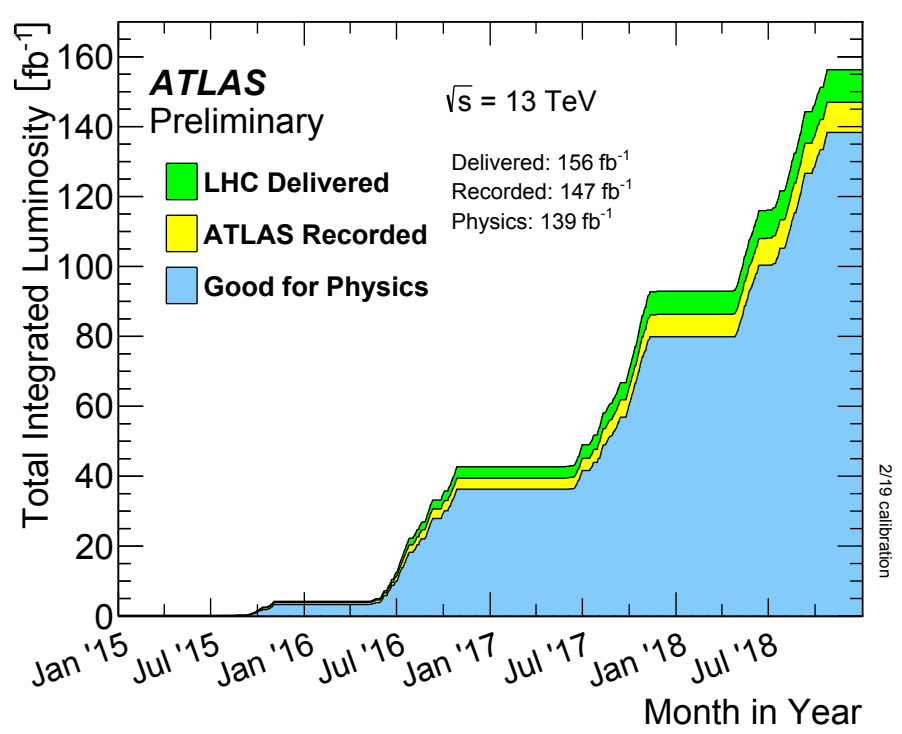

Figura 2.13: Luminosidad total integrada entregada por el LHC (verde), registrada por ATLAS (amarillo) y disponible para análisis físicos (azul) para todo el Run-2.

- Al menos un muón con $p_{\mathrm{T}}>100 \mathrm{GeV}$ y $|\eta|<2.6$, o dos muones con $p_{\mathrm{T}}>20$ $\mathrm{GeV}$ y $|\eta|<2.6$.

- $\mathrm{Al}$ menos un fotón con $p_{\mathrm{T}}>100 \mathrm{GeV}$ y $|\eta|<2.6$, o dos fotones con $p_{\mathrm{T}}>50$ $\mathrm{GeV}$ y $|\eta|<2.6$.

En base a esta selección se reducen significativamente los datos a analizar, asegurando al mismo tiempo disponer de todos los eventos en el estado final buscado.

\subsection{Toma de datos durante el Run-2}

El LHC fue operado durante el Run-2 con una energía de centro de masa de $\sqrt{s}=13 \mathrm{TeV}$ y una luminosidad integrada de $156 \mathrm{fb}^{-1}$ [72]. ATLAS registró 147 $\mathrm{fb}^{-1}$ del total de los datos entregados por el LHC, dando un eficiencia en la toma de datos del $94 \%$. La luminosidad integrada disponible para los análisis físicos llevados a cabo por ATLAS corresponde a 139 (138.9) $\mathrm{fb}^{-1}$, como puede verse en la Figura 2.13.

Las luminosidades instantáneas máximas se incrementaron en un factor cuatro durante los cuatro años del Run-2, resultando en un aumento en el número promedio de interacciones por cruce de paquetes, $\langle\mu\rangle$. Los valores anuales de luminosidad instantánea máxima, $\langle\mu\rangle$ y luminosidad integrada después de requerir condiciones de haz estables y un detector funcional se resumen en la Tabla 2.1 para colisiones $p p$. La incerteza en la luminosidad integrada combinada para 2015-2018 es $1.7 \%$ [72], obtenida usando el detector LUCID-2 [73]. En 2017 se alcanzó el máximo de $\langle\mu\rangle=80$ sólo en unos pocos runs especiales, por lo que el valor máximo para los análisis de física se considera con $\langle\mu\rangle=60$.

El promedio de las interacciones por cruce de paquetes durante los años del Run-2, se muestran en la Figura 2.14.

Vale la pena en este punto definir el concepto de pile-up, íntimamente relacionado con lo anterior, y de gran importancia en los estudios realizados en esta tesis. 
Tabla 2.1: Los valores anuales de luminosidad instantánea máxima $(\mathbf{L})$, interacciones por cruce de paquetes máximo y promedio $(\langle\mu\rangle)$, y la luminosidad integrada para la toma de datos $p p$.

\begin{tabular}{ccccc}
\hline Año & Max. L $\left[\mathbf{c m}^{-2} \mathbf{s}^{-1}\right]$ & Max. $\langle\mu\rangle$ & Media $\langle\mu\rangle$ & $\int \mathbf{L d t}\left[\mathbf{f b}^{-1}\right.$ ] \\
\hline 2015 & $0.5 \times 10^{34}$ & 15 & 13.4 & 3.2 \\
2016 & $1.4 \times 10^{34}$ & 45 & 25.1 & 32.9 \\
2017 & $2.1 \times 10^{34}$ & 80 & 37.8 & 43.9 \\
2018 & $2.1 \times 10^{34}$ & 60 & 36.1 & 58.5 \\
\hline
\end{tabular}

Las trayectorias de las partículas medidas por el detector deben ser asociadas a los vértices de la dispersión dura, es decir, a las coordenadas espaciales donde colisionaron los protones que las originaron. La correcta asociación trayectoria/vértice es de suma importancia para la reconstrucción del evento. Al mismo tiempo, en cada cruce de paquetes se espera que existan múltiples colisiones de protones, que suelen ser interacciones de bajo momento, las que generan vértices primarios adicionales al de la dispersión dura en el evento y, por lo tanto, un desafío mayor para la correcta asociación. A esta superposición de colisiones en un mismo cruce de paquetes de protones, se la conoce como in-time pile-up [74]. Existe además una complejidad extra debida a la posibilidad de que se superpongan en el detector señales pertenecientes a colisiones de protones de paquetes vecinos (recordar que el cruce de paquetes se da cada $25 \mathrm{~ns}$ ), generando lo que se conoce como out-of-time pile-up. Una medida cuantitativa del pile-up y la actividad en el evento, es el número promedio de interacciones inelásticas por cruce de paquetes $\mu$, que sigue una distribución de Poisson con media $\langle\mu\rangle$.

Con respecto a la calidad de los datos colectados por el sistema de TDAQ, ésta se controla constantemente durante la toma de datos, tanto de forma remota

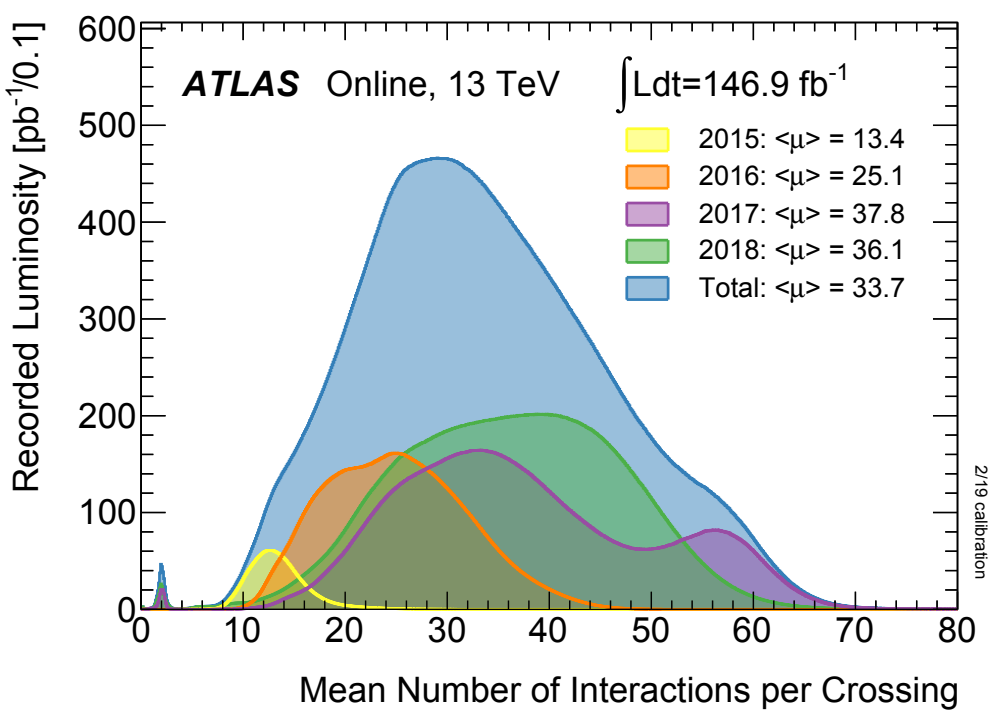

Figura 2.14: Evolución de la media del número de interacciones por cruce de paquetes durante el Run-2. 
como desde la sala de control del detector ${ }^{2}$, donde al menos ocho miembros de la colaboración se encargan de controlar cada aspecto del detector y de la toma de datos en turnos de ocho horas ${ }^{3}$. Las condiciones de la toma de datos se guardan con un intervalo de tiempo del orden de un minuto, que define el bloque de luminosidad (LB). Se excluyen de los análisis los datos de un período de tiempo en el que el detector no ha funcionado perfectamente, y se crea una Good Runs List (GRL), que contiene los LBs de cada ejecución en la que se tomaron los datos con buenas condiciones del detector [75]. Los análisis sólo usan eventos contenidos en estas GRLs.

\subsection{Simulaciones Monte Carlo}

El uso de simulaciones MC en física de altas energías es indispensable. Se utilizan para diversas tareas, entre las que se pueden mencionar la generación de los modelos teóricos que representan la señal en las distintas búsquedas, la generación de procesos del SM con los que comparar los resultados, para obtener incertezas sistemáticas o estudiar el rendimiento del detector, entre otras. Las muestras específicas utilizadas en cada estudio desarrollado en la presente tesis, junto con el detalle del proceso puntual que se genera, se detalla en las correspondientes (y siguientes) secciones. Sin embargo, de manera general, se aclara aquí que la simulación de eventos de colisión que se utiliza, incluye el efecto de múltiples interacciones de protón-protón en el mismo cruce de paquetes o en cruces vecinos. La simulación de colisiones de pile-up se realizó con PYTHia 8.186 utilizando el conjunto de parámetros ajustados por la colaboración ATLAS A3 [76] y el conjunto NNPDF23LO de PDFs. Estos eventos se ponderaron de tal forma de reproducir el número promedio de interacciones de pile-up por cruce de paquetes observado en los datos.

Los eventos generados fueron pasados a través de una simulación completa del detector [77] basada en GEANT 4 [78].

\footnotetext{
${ }^{2}$ https://atlas.cern/tags/control-room

${ }^{3}$ Durante las estadías en el laboratorio, requeridas para ser un miembro activo de la colaboración, he realizado más de 50 turnos de control encargado o de controlar el sistema de trigger o de llevar adelante el run de la toma de datos.
} 


\section{Capítulo 3}

\section{Reconstrucción e identificación de objetos físicos en ATLAS}

Las partículas (y/o los productos de sus decaimientos) generadas en cada colisión, interactúan con el detector de manera característica de acuerdo a su naturaleza. La información proveniente de todos los subdetectores descriptos en el capítulo anterior, permite la reconstrucción e identificación de los objetos físicos presentes en cada evento aceptado por el trigger. El conocimiento de la respuesta del detector a las distintas partículas permite realizar este proceso, que se describe a continuación para todos los objetos utilizados en esta tesis.

\subsection{Fotones y Electrones}

En esta sección se hace una descripción de la reconstrucción e identificación de fotones y electrones ya que agrupan características similares en su tratamiento, priorizando los detalles para los primeros, objetos principales de esta tesis.

\subsubsection{Reconstrucción}

La reconstrucción offline de fotones y electrones [79], utiliza clusters de depósitos de energía dinámicos de tamaño variable medidos en celdas de los calorímetros electromagnético y hadrónico, conectadas topológicamente [80], llamados topo-clusters. Esta es la diferencia principal introducida en el Run-2, que al no depender de clusters de tamaño fijo, permite recuperar eficientemente la energía de fotones bremsstrahlung o de electrones provenientes de fotones convertidos.

Después de aplicar las correcciones de la posición y calibraciones de energía a los topo-clusters, se buscan coincidencias entre éstos y trazas del ID (reajustadas para tener en cuenta el bremsstrahlung), siguiendo el procedimiento descripto en [81], para reconstruir los candidatos a electrones. Los topo-clusters que no coinciden con ninguna traza o vértices de conversión, son reconstruidos como candidatos a fotones no convertidos. Aquellos topo-clusters sin trazas, pero que estén asociados a un vértice de conversión, es decir que sean consistentes con la hipótesis de un proceso de conversión $\gamma \rightarrow e^{+} e^{-}$, son clasificados como candidatos a fotón convertido. En algunos casos, un objeto puede ser reconstruido tanto como un electrón y como un fotón, debido a la similitud entre las señales de estos objetos (especialmente con fotones 
convertidos), para maximizar la eficiencia de su reconstrucción. El tratamiento de estos casos se discute en la Sección 5.1.5.

\subsubsection{Identificación}

La identificación de los candidatos a fotones en ATLAS se basa en requisitos de selección rectangulares basados en un conjunto de variables calorimétricas, que describen su depósito de energía en forma de lluvias electromagnéticas, y proporcionan una buena discriminación para los candidatos a fotones aislados. Los candidatos a electrones se seleccionan en base a un método de likelihood (LH) [82, 79], el cual utiliza las características de los depósitos de energía calorimétricos (en sus distribuciones de forma laterales y longitudinales), la calidad de la traza, la concordancia entre traza y cluster, y la identificación provista por el TRT. Las pdf del LH se obtienen en base a eventos de decaimientos de $\mathrm{J} / \Psi$ y $\mathrm{Z}$ para electrones de bajo y alto $p_{\mathrm{T}}$ respectivamente como se describe en [83]. Los umbrales de discriminación se ajustan también linealmente en función del número de vértices reconstruidos para obtener un rechazo estable de los objetos de fondo. Se determinaron tres puntos de operación o Working points (WPs), correspondientes a un aumento en el umbral del valor del discriminante del LH con eficiencias de identificación de $93 \%$ (loose), $88 \%$ (medium) y $80 \%$ (tight).

Las variables mencionadas utilizadas para la identificación se muestran en la Tabla 3.1, omitiendo aquellas relacionadas con el ID, que son sólo utilizadas para la identificación de electrones, permitiendo centrar la discusión en las calorimétricas, utilizadas para ambos tipos de objetos y que serán también claves en la discusión de las eficiencias del trigger de fotones en el Capítulo 4.

Una de las características que tienen estas cantidades, que representan depósitos de energía longitudinal y lateral en las diferentes capas del calorímetro electromagnético, es su alto poder de separación entre fotones provenientes de la dispersión dura y fotones falsos. Estos fotones falsos pueden ser el resultado de fotones que se originan a partir del decaimiento de hadrones neutros en jets, o de jets que depositan una gran fracción de energía en el calorímetro EM. Estos últimos realizan depósitos de energía extendidas en un mayor número de celdas, en comparación con los fotones reales cuya distribución está topológicamente concentrada en un menor número de celdas. La eficiente discriminación entre ambos casos es la principal tarea de los algoritmos de identificación.

Para fotones se definen tres Working points: loose, medium y tight. Los WPs de identificación son estrictamente inclusivos, es decir, fotones que satisfacen la selección tight son un subconjunto de los que satisfacen la selección medium, contenidos a su vez en la selección loose. En la Tabla 3.2 se muestran cuáles de las variables 3.1, se usan para la identificación de fotones (para más detalle véase [84]). La selección loose se basa en la forma de las lluvias en la segunda capa del calorímetro EM y en la energía depositada en el calorímetro hadrónico. La selección medium agrega un corte en la variable $E_{\text {ratio }}$. Además de los criterios de selección medium, la selección tight utiliza información de la primera capa finamente segmentada del calorímetro. Es importante aclarar que la identificación medium para fotones sólo está disponible online, y se agrega en esta descripción por su utilización en el cálculo de las eficiencias del trigger que se detalla en el próximo capítulo. Durante el análisis offline, sólo se cuenta con fotones loose o tight. 


\begin{tabular}{|c|c|c|}
\hline Tipo & Descripción & Nombre \\
\hline \multirow[t]{2}{*}{ Fuga hadrónica } & $\begin{array}{l}\text { razón entre la } E_{\mathrm{T}} \text { en la primera capa del HCAL y la } E_{\mathrm{T}} \text { del cluster } \\
\text { electromagnético (si }|\eta|<0.8 \text { y }|\eta|>1.37) \text {. }\end{array}$ & $R_{\text {Had1 }}$ \\
\hline & $\begin{array}{l}\text { razón entre la } E_{\mathrm{T}} \text { en todo el HCAL y la } E_{\mathrm{T}} \text { del cluster electromagnético } \\
\text { (si }|\eta|>0.8 \text { y }|\eta|<1.37 \text { ). }\end{array}$ & $R_{\mathrm{Had}}$ \\
\hline ECAL $\left(3^{r a}\right.$ capa $)$ & razón entre la energía en la $3^{r a}$ capa y la energía total. & $f_{3}$ \\
\hline \multirow[t]{3}{*}{ ECAL $\left(2^{d a}\right.$ capa $)$} & $\begin{array}{l}\text { ancho lateral de la lluvia, } \sqrt{\left(\Sigma E_{i} \eta_{i}^{2}\right) /\left(\Sigma E_{i}\right)-\left(\left(\Sigma E_{i} \eta_{i}\right) /\left(\Sigma E_{i}\right)\right)^{2}} \text {, donde } \\
E_{i} \text { es la energía y } \eta_{i} \text { es la pseudorapidez de la celda } i \text { y la suma se calcula } \\
\text { dentro de una ventana de } 3 \times 5 \text { celdas. }\end{array}$ & $W_{\eta}$ \\
\hline & $\begin{array}{l}\text { razón entre la suma de la energía en } 3 \times 3 \text { celdas y la energía en } 3 \times 7 \text { celdas } \\
\text { centradas en la posición del cluster. }\end{array}$ & $R_{\eta}$ \\
\hline & $\begin{array}{l}\text { razón entre la energía en } 3 \times 7 \text { celdas y la energía en } 7 \times 7 \text { celdas centradas } \\
\text { en la posición del cluster. }\end{array}$ & $R_{\phi}$ \\
\hline \multirow[t]{6}{*}{ ECAL $\left(1^{r a}\right.$ capa $)$} & $\begin{array}{l}\text { ancho lateral total de la lluvia, } \sqrt{\left(\Sigma E_{i}\left(i-i_{\max }\right)^{2}\right)\left(\Sigma E_{i}\right)} \text {, donde } i \text { va sobre } \\
\text { todas las strips en una ventana de } \Delta \eta \times \Delta \phi \approx 0.0625 \times 0.2 \text {, correspon- } \\
\text { diendo típicamente a } 20 \text { strips en } \eta \text { y } i_{\max } \text { es el índice de la strip con } \\
\text { energía más alta. }\end{array}$ & $W_{\text {stot }}$ \\
\hline & $\begin{array}{l}\text { ancho de la lluvia, } \sqrt{\left(\Sigma E_{i}\left(i-i_{\max }\right)^{2}\right)\left(\Sigma E_{i}\right)} \text {, donde } i \text { va sobre todas las } \\
\text { strips en una ventana de } 3 \times 2 \eta \times \phi \text { strips, y } i_{\max } \text { es el índice la strip con } \\
\text { energía más alta. }\end{array}$ & $w_{s 3}$ \\
\hline & $\begin{array}{l}\text { razón entre la diferencia de energía entre el máximo y el segundo máximo } \\
\text { depósito de energía en el clúster y la suma de esas energías. }\end{array}$ & $E_{\text {ratio }}$ \\
\hline & razón entre la energía de la $1^{r a}$ capa y la energía total. & $f_{1}$ \\
\hline & $\begin{array}{l}\text { fracción de energía fuera de un núcleo de tres strips centrales y dentro } \\
\text { de siete strips. }\end{array}$ & $F_{\text {side }}$ \\
\hline & $\begin{array}{l}\text { Diferencia entre la energía asociada con el segundo máximo en la } 1^{\text {ra }} \\
\text { capa, y la energía reconstruida en la strip con el mínimo valor entre el } \\
\text { primer y segundo máximo. }\end{array}$ & $\Delta E$ \\
\hline
\end{tabular}

Tabla 3.1: Definición de las variables discriminatorias de fotones.

En base a una colección de fotones radiados de leptones provenientes del decaimiento de un bosón $Z$, con $E_{\mathrm{T}}>25 \mathrm{GeV}$, se determina una eficiencia integrada sobre los conjuntos de datos de la selección loose (tight) de 98,9\% (87,5\%) para fotones no coincidentes con ninguna traza y de $96,3 \%(87,6 \%)$ para fotones coincidentes con vértices de conversión [79].

\begin{tabular}{|l||l|l|l|}
\hline \multicolumn{1}{|c||}{} & \multicolumn{3}{c|}{ Fotón ID } \\
\hline Nombre & loose & medium & tight \\
\hline$R_{\text {Had1 }}$ & $\checkmark$ & $\checkmark$ & $\checkmark$ \\
\hline$w_{s 3}$ & & & $\checkmark$ \\
\hline$W_{\eta}$ & $\checkmark$ & $\checkmark$ & $\checkmark$ \\
$R_{\eta}$ & $\checkmark$ & $\checkmark$ & $\checkmark$ \\
$R_{\phi}$ & & & $\checkmark$ \\
\hline$W_{\text {stot }}$ & & & $\checkmark$ \\
$E_{\text {ratio }}$ & & $\checkmark$ & $\checkmark$ \\
$f_{1}$ & & & $\checkmark$ \\
$\Delta E$ & & & $\checkmark$ \\
$F_{\text {side }}$ & & & $\checkmark$ \\
\hline
\end{tabular}

Tabla 3.2: Variables usadas para los diferentes WPs de identificación de fotones. La identificación medium para los fotones está disponible sólo online. 


\section{Descripción de variables de identificación calorimétricas}

Es importante ampliar la discusión sobre algunas de las variables calorimétricas empleadas para la identificación de fotones, debido a que son utilizadas en el Capítulo 4 para definir los candidatos non-tight, en el marco del método ABCD para sustracción de fondo. De la lista de variables (Tabla 3.1), se detallan a continuación aquellas que utilizan la primera capa del calorímetro electromagnético finamente segmentada, que está compuesta por bandas strips, y en particular proveen la base para la separación entre fotones aislados y los fotones provenientes del decaimiento de piones neutros, como se ve en los perfiles de deposición de las lluvias para ambos procesos en la Figura 3.1.
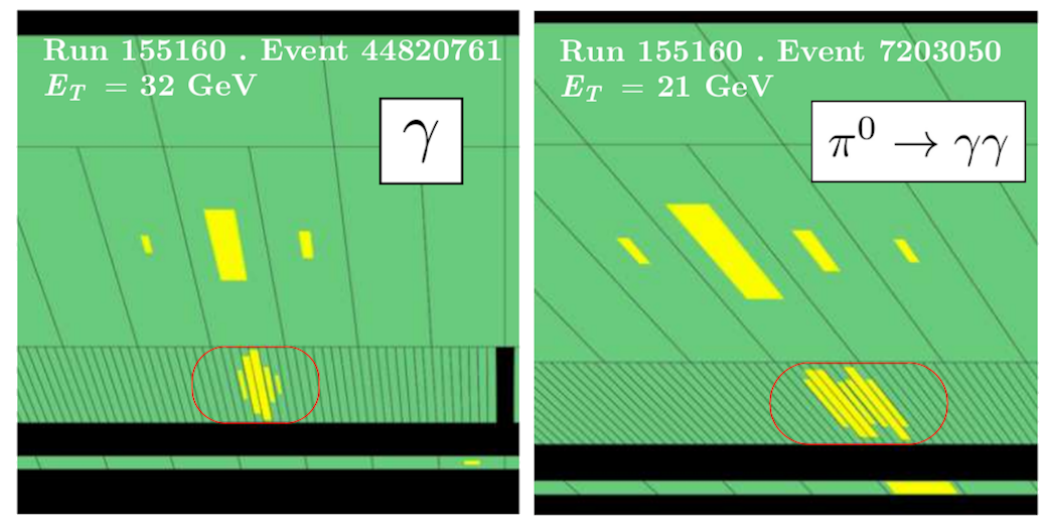

Figura 3.1: Deposiciones características para un fotón aislado (izquierda) y para un $\pi^{0} \rightarrow \gamma \gamma$ (derecha) en datos.

- RMS del perfil lateral de energía en $\eta$ (3 bandas)

$$
w_{s, 3}=\sqrt{\frac{\sum E_{i}\left(i-i_{\max }\right)^{2}}{\sum E_{i}}}
$$

mide el ancho pesado de la lluvia electromagnética a lo largo de $\eta$ en la primera capa del calorímetro EM considerando sólo tres bandas, la banda con mayor deposición y sus dos primeras vecinas.

- Perfil lateral de energía en $\eta$

$$
F_{\text {side }}=\frac{E( \pm 3)-E( \pm 1)}{E( \pm 1)}
$$

es la fracción de energía fuera del núcleo central de tres celdas, pero dentro de las siete celdas vecinas. $E( \pm n)$ es la energía en las $\pm n$ celdas alrededor de aquella con la mayor deposición.

- Diferencia al segundo máximo

$$
\Delta E=\left[E_{2^{d o} \max }-E_{\min }\right]
$$

es la diferencia entre la energía de la celda asociada con el segundo máximo, y la energía reconstruida en la celda con el menor valor entre los dos primeros máximos locales $E_{\mathrm{min}}$. En caso de no haber segundo máximo se fija $\Delta E=0$. 
- Asimetría de los dos máximos locales en $\eta$

$$
E_{\text {ratio }}=\frac{E_{1^{e r} \max }-E_{2^{\text {do }} \max }}{E_{1^{e r} \max }+E_{2^{\text {do }} \max }}
$$

mide la diferencia relativa entre las energías de las dos celdas con máxima deposición. En caso de no haber segundo máximo se fija $E_{\text {ratio }}=1$.

Distintas distribuciones de estas variables se pueden ver en [41] para fotones reales y para jets de QCD que representan el fondo.

\subsubsection{Aislamiento}

Para reducir los fondos de los jets mal identificados como fotones y del decaimiento de hadrones dentro de los jets (como el caso de piones neutros mencionado en la sección anterior), se requiere aislar a los candidatos a fotones, es decir, identificarlos con su energía concentrada en un conjunto de celdas sin actividad calorimétrica a su alrededor, para el caso del aislamiento calorimétrico, y de manera similar para las trazas.

La variable de aislamiento calorimétrico $E_{\mathrm{T}}^{\text {iso }}\left(E_{T}^{\text {topoetcone } 40}\right)$ se calcula como la suma de las energías transversales en las celdas de los topo-clusters, en un cono de radio $\mathrm{R}$ (en el plano $\eta-\phi$ ) alrededor del candidato. En este caso con $\Delta R<0.4$. La energía depositada por el fotón y las contribuciones del pile-up se restan evento a evento [85]. En particular, la contribución del candidato se elimina al ignorar la región rectangular de $N_{\eta} \times N_{\phi}=5 \times 7$ celdas, en el centro del cono. En la Figura 3.2 , se muestra de forma esquemática cómo se construye la variable de aislamiento calorimétrico.

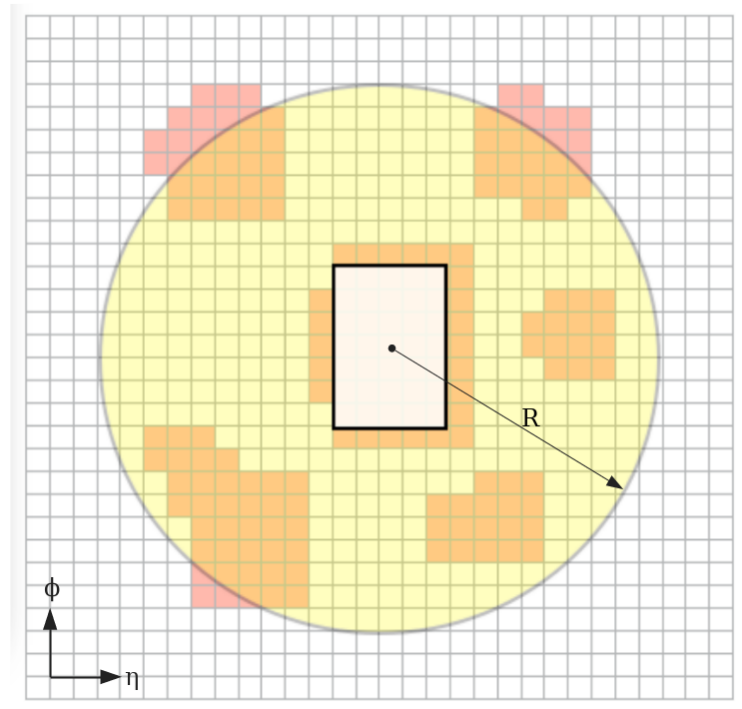

Figura 3.2: Esquema ilustrando el cálculo de la energía de aislamiento calorimétrico del fotón. Se construye un cono de radio $\mathrm{R}$ alrededor del candidato y se suman las energías transversales de los topo-clusters en los calorímetros, sin incluir la energía depositada por el fotón candidato.

La variable de aislamiento de trazas, $p_{\mathrm{T}}^{\text {iso }}\left(p_{T}^{\text {varcone } 20}\right)$, se obtiene sumando escalarmente el $p_{\mathrm{T}}$ de las trazas de buena calidad en un cono alrededor del candidato (en 
este caso se toma $\left.\Delta R^{\mathrm{var}}<0.2\right)$. Se define, en este contexto, a una traza como buena si tiene $p_{\mathrm{T}}>1 \mathrm{GeV}$, con una distancia mínima al vértice primario a lo largo del eje del haz $\left|z_{0} \sin \theta\right|<3 \mathrm{~mm}$, y se excluyen las trazas asociadas con la conversión de fotones o las del candidato a leptón.

Para electrones se definen dos estrategias distintas de aislamiento, una con el objetivo de obtener un valor constante en la eficiencia y otro con valores fijos en las variables de selección. El WP llamado FCLoose, esta diseñado para obtener eficiencias uniformes (en distintos rangos de $p_{\mathrm{T}}$ ), mayores al $90 \%$, para todo valor de $\eta$. Otros tres WP tienen requerimientos fijos en las variables de aislamiento calorimétricas y/o de trazas.

La implementación de los distintos criterios de aislamiento se decide en base a las necesidades físicas de cada análisis en particular, ya que las distintas recomendaciones son el resultado de un compromiso entre una identificación eficiente de los objetos buscados y el rechazo de otras partículas erróneamente identificadas.

Las definiciones exactas de los WPs utilizados en esta tesis se muestran en la Tabla 3.3 y se describen en detalle en [79, 86].

Tabla 3.3: Definición de los WP de aislamiento utilizados en la presente tesis para fotones y electrones.

\begin{tabular}{llcc}
\hline Objeto & WP & Aislamiento Calorimétrico & Aislamiento de Trazas \\
\hline Fotón & FixedCutTightCaloOnly & $E_{\mathrm{T}}^{\text {iso }}(\Delta R<0.4)<0.022 \cdot E_{\mathrm{T}}+2.45 \mathrm{GeV}$ & \\
& FCTight & $E_{\mathrm{T}}^{\text {iso }}(\Delta R<0.4)<0.022 \cdot E_{\mathrm{T}}+2.45 \mathrm{GeV}$ & $p_{\mathrm{T}}^{\text {iso }}\left(\Delta R^{\mathrm{var}}<0.2\right) / p_{\mathrm{T}}<0.05$ \\
Electrón & FCLoose & $E_{\mathrm{T}}^{\text {iso }}(\Delta R<0.2) / E_{\mathrm{T}}<0.2$ & $p_{\mathrm{T}}^{\text {iso }}\left(\Delta R^{\mathrm{var}}<0.2\right) / p_{\mathrm{T}}<0.15$ \\
\hline
\end{tabular}

\section{2. $\quad$ Muones}

La reconstrucción de los muones se hace, en primer lugar, de manera independiente en el ID y en el MS. Luego, la información proveniente de los distintos subdetectores, incluyendo a los calorímetros, se combina para formar los muones que se usan en los análisis [86, 87]. La combinación de información de distintos subdetectores define cuatro tipos de muones, dependiendo de la información utilizada para su reconstrucción:

- Muones Combinados (CB): su traza es una combinación, vía un re-ajuste global, de los hits del ID y el MS. Representan la mayoría de los candidatos a muones y tienen la mejor resolución de momento.

- Muones Segmentados (ST): una traza en el ID es identificada como un muón, si al extrapolarla hasta el MS, puede ser asociada con al menos un segmento de traza en las cámaras MDT o CSC.

- Muones Calorimétricos (CT): una traza en el ID es identificada como un muón, si puede ser asociada con un depósito de energía el calorímetro compatible con una partícula mínimamente ionizante. Los muones CT se optimizan para $15<p_{\mathrm{T}}<100 \mathrm{GeV}$ en la región $|\eta|<0.1$, donde el MS está parcialmente instrumentado.

- Muones Extrapolados (ME): la trayectoria del muón se reconstruye usando sólo las trazas del MS y pidiendo un requerimiento loose en su compatibilidad 
con el punto de interacción original. Los muones ME se usan para extender la aceptancia en la región $2.5<|\eta|<2.7$, no cubierta por el ID.

El solapamiento entre diferentes tipos de muones se resuelve de la siguiente manera. Cuando dos tipos de muones comparten la misma traza del ID, se prefieren los $\mathrm{CB}$, luego los ST y finalmente los muones CT. El solapamiento con muones ME se resuelve analizando los hits de las trazas, seleccionando las trazas con mejor ajuste y mayor número de hits.

Como parte de la identificación de muones se aplican cortes de calidad que distingan muones aislados de aquellos provenientes de procesos de fondo, principalmente del decaimiento de piones y kaones. Las variables con buen poder de discriminación utilizadas se describen en [87]. Se definen cuatro selecciones de identificación Loose, Medium, Tight, y High- $p_{\mathrm{T}}$, siendo las primeras tres categorías inclusivas, y Medium la selección por defecto en ATLAS. Por último, a los candidatos a muones que serán utilizados por los análisis se les pide que satisfagan los requerimientos de aislamiento, tanto a nivel de trazas como calorimétrico, de manera análoga a lo que se detalló para fotones en la sección anterior. Para el primer caso, se usa una variable similar a la usada por fotones, pero con un cono de radio variable $\left(\Delta R=\min \left(10 \mathrm{GeV} / p_{T}^{\mu}, 0.3\right)\right)$ alrededor del momento transverso del muón $p_{T}^{\mu}$, excluyendo la traza del muón. Para el aislamiento calorimétrico se usa la misma variable $E_{\mathrm{T}}^{\text {iso }}$, con la diferencia de $R=0.2$, en vez de 0.4 como antes. En base a estas variables se definen 7 criterios de selección de aislamiento (7 WPs), optimizados para diferentes análisis.

\subsection{Jets}

Los jets o "chorros de partículas" son el resultado de la hadronización de quarks y gluones generados en la dispersión dura. Los jets se reconstruyen utilizando el algoritmo anti- $k_{t}$ [88] con radio $R=0.4$, y se asocian a las colecciones de objetos AntiKt4EMTopoJets o AntiKt4EMPFlowJets, dependiendo de qué información del detector utilizan para la reconstrucción. En lo que resta de la presente tesis, se refiere a dichas colecciones simplemente como EMTopo o PFlow jets. La primera se reconstruye a partir de clusters topológicos de celdas calorimétricas [89], donde los clusters se forman a partir de celdas con energías significativamente superiores al nivel de ruido [80] y calibradas a la escala electromagnética. La segunda colección toma como entrada trazas de partículas cargadas que coinciden con el vértice primario con el requerimiento de $\left|z_{0} \sin \theta\right|<2.0 \mathrm{~mm}$ y los clusters del calorímetro que satisfacen el algoritmo de sustracción de energía que elimina los depósitos calorimétricos provenientes de trazas de buena calidad de cualquier otro vértice [90]. En particular, los topo-clusters se forman tomando inicialmente las celdas con una relación señal a ruido de 4 veces. Las celdas vecinas se suman a las anteriores si la relación es al menos de 2 veces y finalmente se agrega una última capa de celdas que rodean a las anteriores. Una vez que se definen los topo-clusters, se pasan como entrada a los algoritmos de recombinación secuencial, como el ya mencionado anti- $k_{t}$, para la reconstrucción de los jets. La combinación de cada par de objetos (topo-clusters) se basa en una distancia $d_{i j}$ definida como:

$$
d_{i j}=\min \left(p_{T, i}^{2 p}, p_{T, j}^{2 p}\right) \frac{\Delta R_{i j}^{2}}{R^{2}}
$$


siendo $\Delta R_{i j}=\sqrt{\left(\phi_{i}-\phi_{j}\right)^{2}+\left(\eta_{i}-\eta_{j}\right)^{2}}, d_{i, B}$ la distancia entre el cluster i y el haz, el momento transverso del cluster es $p_{T, i}$, y p es un parámetro que define el algoritmo a utilizar, siendo - 1 para el anti- $k_{t}$. Como ya se mencionó, los jets que se utilizan en la presente tesis, utilizan $R=0.4$, siendo $\mathrm{R}$ un parámetro asociado al radio del cono de los jets reconstruidos. Con esto, la forma de proceder del algoritmo es la siguiente: primero se obtiene un conjunto de todas las posibles distancias. Si el elemento más pequeño del conjunto es $d_{i, B}$, el cluster se convierte en un jet y no se lo tiene en cuenta en lo que sigue. Si el elemento más pequeño es $d_{i, j}$, los clusters i y j se combinan, asignando como nuevo $p_{\mathrm{T}}$ la suma de los momentos de sus componentes, y se procede a generar un nuevo conjunto de distancias, donde el nuevo objeto combinado reemplaza a i y j. Este proceso continua hasta que todos los clusters son clasificados.

Estos jets se calibran con una serie de operaciones en cadena que primero corrigen la contaminación por pile-up, y luego corrigen la respuesta energética promedio reconstruida del jet para que coincida con la de los jets formados a partir de partículas estables después de la hadronización. Las correcciones incluyen una Jet Energy Scale (JES) o escala de energía del jet basada en MC, una global sequential calibration (GSC) o calibración secuencial global para corregir las diferencias adicionales en la distribución de energía, en la forma de las lluvias calorimétricas del jet y el sabor del partón inicial además de mejorar la resolución, y una corrección final in situ aplicada sólo a los datos [91]. Las resoluciones de los jets se corrigen aplicando un smearing gaussiano a los jets simulados, cuya resolución es menor que la de los jets en los datos.

Las incertezas relativas a la escala y la resolución de la energía de los jets se derivan en función de $p_{\mathrm{T}} \mathrm{y} \eta$. Se determinan utilizando una combinación de datos y $\mathrm{MC}$, comparando eventos di-jet, $\mathrm{Z}+$ jet y $\gamma+$ jet.

Para reducir la contribución del pile-up y mejorar la pureza de los jets reconstruidos, se utiliza una combinación multivariable de JVF (Jet Vertex Fraction, definida como la suma escalar del momento transverso de las trazas asociadas con un jet y provenientes del vértice de la dispersión dura dividida por la suma escalar del $p_{\mathrm{T}}$ de todas las trazas asociadas al jet) y $N_{V t x}$ (número de vértices primarios en el evento), llamada JVT o Jet Vertex Tagger, que provee además una eficiencia del rechazo de fondo estable frente al $N_{V t x}$ [92].

La reconstrucción con PFlow (Flujo de Partículas) permite combinar la resolución energética óptima del detector de trazas y del calorímetro en los regímenes de baja y alta energía. La colaboración de ATLAS ha reportado mejoras tanto en jets [90] como en $E_{\mathrm{T}}^{\text {miss }}$ [93] usando este algoritmo. Además, los jets de PFlow han mostrado incertezas significativamente menores para jets en el régimen de bajo $p_{\mathrm{T}}$, debido a la mejor estimación de la energía del jet lograda añadiendo la información de las trazas. Se espera que este algoritmo sea utilizado por defecto en los estudios futuros dentro de la colaboración

PFlow introduce un enfoque alternativo, en el que las mediciones tanto del detector de trazas como del calorímetro se combinan para formar las señales, que idealmente representan partículas individuales. La energía depositada en el calorímetro por todas las partículas cargadas es eliminada. La reconstrucción del jet se realiza entonces en un conjunto de "objetos del flujo de partículas" que consisten en la energía calorimétrica restante y en trazas que coinciden con la dispersión dura.

De las Ecuaciones 2.6 y 2.7 para la resolución en energía del detector interno 
y el calorímetro, se desprende que para las partículas cargadas de baja energía, la resolución del momento del detector de trazas es significativamente mejor que la resolución energética del calorímetro. Además, debido a que en el ID se reconstruyen partículas cargadas con $p_{\mathrm{T}}>400 \mathrm{MeV}$, cuyos depósitos de energía suelen no superar los umbrales de ruido requeridos para crear los topo-clusters, la aceptancia del detector aumenta de esta forma.

Cuando se reconstruye una traza, se puede determinar si está asociada a un vértice y, en caso afirmativo, el vértice del que se origina. Por lo tanto, en presencia de múltiples interacciones de pile-up in-time, el efecto de las partículas adicionales puede mitigarse rechazando las señales originadas en los vértices de pile-up. Y esto a su vez, se complementa con la capacidad del calorímetro para reconstruir tanto las partículas cargadas como las neutras. A altas energías, la resolución energética del calorímetro es superior a la resolución del momento del detector de trazas, por lo que se utiliza una combinación de los dos subdetectores para una reconstrucción óptima del evento. Para $|\eta|>2.5$, sólo está disponible la información del calorímetro, con lo que en esta región, sólo los topo-clusters se utilizan para la reconstrucción del PFlow jet.

Sin embargo, PFlow introduce una complicación. Para cualquier partícula cuya medición de la traza debe ser usada, es necesario identificar correctamente su depósito en el calorímetro, para evitar el doble conteo de su energía en la reconstrucción. El algoritmo de PFlow toma una decisión booleana sobre si se debe utilizar el detector de trazas o la medición del calorímetro. Si se va a utilizar la medición del detector de trazas de una partícula, se debe restar la energía correspondiente de la medición del calorímetro. La capacidad de restar con precisión toda la energía de una sola partícula, sin eliminar la energía depositada por ninguna otra partícula, constituye el criterio de rendimiento clave sobre el que se optimiza el algoritmo.

\subsubsection{Jets provenientes de quarks b: b-jets}

La identificación de jets originados por quarks b, utiliza la relativa larga vida de estos quarks (del orden de $1.5 \mathrm{ps}$ ), que genera un desplazamiento de algunos milímetros del vértice de decaimiento respecto del vértice primario, la alta multiplicidad del decaimiento y la gran masa de los b-hadrones, en comparación con los gluones y los quarks livianos [94]. Un jet anti- $k_{t}$ de $R=0.4$ se considera o se etiqueta como un b-jet, si pasa los requerimientos del algoritmo MV2[95] en el punto de trabajo (WP) de $77 \%$ de eficiencia de etiquetado correcto, habiendo satisfecho las selecciones $p_{\mathrm{T}}>20 \mathrm{GeV},|\eta|<2.5$ y pasado el corte en JVT. Este algoritmo clasificador multivariable utiliza un discriminante BDT, boosted decision tree. Es posible utilizar para esta clasificación otro algoritmo desarrollado dentro de la colaboración ATLAS, el DL1 [96], basado en una red neuronal profunda, con similar rechazo a los jets originados por quarks c o de jets livianos (originados por quarks $\mathrm{u}, \mathrm{d}$, s o gluones). Las variables que se utilizan para la clasificación utilizan el parámetro de impacto y la reconstrucción de vértices secundarios. Para el WP de $77 \%$, uno de cada seis c-jets son erróneamente identificados como b-jets, mientras que para jets livianos la tasa de rechazo es de uno de 134 [97]. El cociente entre la eficiencia de etiquetado en datos y MC provee los factores de escala que se utilizan para corregir las (in)eficiencias de seleccionar b-jets simulados con respecto a los evaluados en datos [98]. 


\subsection{Momento transverso faltante}

En el Capítulo 2 se mencionó la motivación, basada en consideraciones de invariancia y conservación, por la que en ATLAS se utilizan las componentes transversales de ciertas cantidades. En particular, la consideración de ausencia de momento transverso de los partones incidentes obliga, vía conservación, a que el momento transverso del estado final sea también cero. Es decir que si sumamos vectorialmente el momento de todos los objetos presentes en el evento, el resultado debe ser cero. Sin embargo, existen razones por las que se dan diferencias con cero: la posibilidad de una mala reconstrucción/calibración de alguno de los objetos presentes y, sobre todo, la posible presencia de partículas invisibles al detector, como pueden ser los neutrinos o más interesante para búsquedas de nueva física, partículas desconocidas que interactúan débilmente con la materia del detector. La variable que mide estas diferencias se la llama momento transverso faltante y está definida como la suma vectorial del momento transverso de todas las partículas observadas en el evento, con signo negativo.

$E_{\mathrm{T}}^{\text {miss }}$ cuantifica entonces, el desbalance de momentos en el plano x-y, con respecto al momento de cada objeto reconstruido en un dado evento. El momento transverso faltante se calcula con un algoritmo basado en objetos [99, 100]. Debido a que una celda del calorímetro puede pertenecer a más de un objeto reconstruido (un electrón y un jet, por ejemplo), se toma un orden para elegir un sólo objeto y así evitar posibles dobles conteos. La preferencia para eliminar el solapamiento, tiene el siguiente orden: electrones, fotones, jets y muones. Una vez que todas las celdas se asignaron unívocamente a un dado objeto, las componentes x e y de $E_{\mathrm{T}}^{\text {miss }}$ se obtienen de la suma:

$$
E_{x(y)}^{\mathrm{miss}}=E_{x(y)}^{\mathrm{miss} e}+E_{x(y)}^{\mathrm{miss} \gamma}+E_{x(y)}^{\text {missjet }}+E_{x(y)}^{\text {miss } \mu}+E_{x(y)}^{\text {miss Soft Term }},
$$

donde cada término se calcula a partir de la suma negativa de los objetos reconstruidos y calibrados en la categoría respectiva.

- $E_{x(y)}^{\text {miss }}{ }^{e}$ se reconstruye a partir de las energías calibradas de los clusters asociados a electrones con $p_{\mathrm{T}}>10 \mathrm{GeV}$ y $0<|\eta|<1.37$ o $1.52<|\eta|<2.47$.

- $E_{x(y)}^{\text {miss } \gamma}$ se reconstruye a partir de las energías calibradas de los clusters asociados a fotones con $p_{\mathrm{T}}>25 \mathrm{GeV}$ y $0<|\eta|<1.37$ o $1.52<|\eta|<2.47$.

- $E_{x(y)}^{\text {miss }{ }^{\mu}}$ se obtiene de la contribución de muones con $p_{\mathrm{T}}>10 \mathrm{GeV}$ y $|\eta|<2.7$.

- $E_{x(y)}^{\text {miss }}$ jets se obtiene de los jets calorimétricos, reconstruidos por el algoritmo anti- $k_{t}$ con un radio $R=0.4$ y un $p_{\mathrm{T}}>20 \mathrm{GeV}$. Para no incluir los jets provenientes del pile-up, se pide JVT $>0.64$ para los jets con $p_{\mathrm{T}}<50 \mathrm{GeV}$ y $|\eta|<2.47$. Las trazas asociadas a jets que no satisfagan este requerimiento se incluyen en Soft Term.

- $E_{x(y)}^{\text {miss SoftTerm }}[101,102]$ se construye a partir de trazas con $p_{\mathrm{T}}>0.4 \mathrm{GeV}$ y $|\eta|<2.5$ que no tienen asociado ningún objeto. Sólo se incluyen trazas compatibles con el vértice primario. 
Una vez obtenidas las componentes x e y, $E_{\mathrm{T}}^{\text {miss }}$ y su ángulo azimutal se calculan como:

$$
\begin{gathered}
E_{\mathrm{T}}^{\mathrm{miss}}=\sqrt{\left(E_{x}^{\mathrm{miss}}\right)^{2}+\left(E_{x}^{\mathrm{miss}}\right)^{2}} \\
\phi^{\text {miss }}=\arctan \left(\frac{E_{x}^{\text {miss }}}{E_{x}^{\text {miss }}}\right)
\end{gathered}
$$

donde $\phi^{\text {miss }}$, más allá de su uso para los análisis físicos, es una variable muy utilizada para estudios de diagnóstico que, en base a su uniformidad, permite asegurar que el detector no introduce ningún sesgo en la dirección de $E_{\mathrm{T}}^{\text {miss }}$. 


\section{Capítulo 4}

\section{Eficiencia del trigger de fotones y multijets}

En este capítulo se describen los métodos utilizados y los resultados obtenidos en el cálculo de las eficiencias de los triggers de fotones y multijets. Los primeros son necesarios para el análisis central de esta tesis que se describe en el Capítulo 5. Los segundos se requieren tanto para la búsqueda de SUSY con multijets y $E_{\mathrm{T}}^{\text {miss }}$ en estado final, como se describe en la Sección 4.4, así como para futuras búsquedas guiadas por modelos de Stealth SUSY, como se detallan en el Capítulo 7.

Para medir la eficiencia de estos triggers en lo que sigue se utiliza un método de tipo data-driven (basados en datos), llamado método Bootstrap en el cual se usan eventos donde los objetos de estudio (fotones o jets) son disparados por un nivel del trigger inferior o por un trigger que no sesgue la muestra con la que se trabaja. Este método es complementario al método del decaimiento radiativo del bosón $Z$, donde se utilizan fotones provenientes del decaimiento radiativo del $Z$ a dos leptones [103]. Este último es estadísticamente limitado debido a la sección eficaz del proceso, y solo permite hacer estudios a bajos valores de $p_{\mathrm{T}}$, sin llegar a cubrir el rango de alto $p_{\mathrm{T}}$ correspondiente al análisis presentado en este trabajo.

\section{1. $\quad$ El método Bootstrap}

El término Bootstrap se utiliza en diversas áreas del conocimiento con significados diferentes pero, en general, refiere a un proceso autocontenido y recursivo, que avanza sin la necesidad de una acción externa. Una de las ideas con que se asoció este término originalmente es la de "Levantarse del suelo, tirando de la correa de tu propia bota". Una acción que es imposible pero que brinda una imagen pictórica a la idea de proceso autocontenido. En particular se suele graficar esta idea con una de las historias del Barón de Munchausen (del libro de Rudolf E. Raspe [104]), que se muestra en la Figura 4.1. Si bien a lo largo de la obra no aparece el término Bootstrap explícitamente, el intento del Barón para salir del pantano tirando de su cabello cumple el mismo propósito.

En el caso de las eficiencias, el método de Bootstrap se refiere a un método datadriven de muestreo recursivo, donde el concepto se acerca a la acepción empleada usualmente en estadística, asociada al trabajo de Bradley Efron [105] y se distancia, por supuesto, del infructífero intento del Barón.

En el método Bootstrap, la eficiencia se calcula como la del HLT respecto de la 


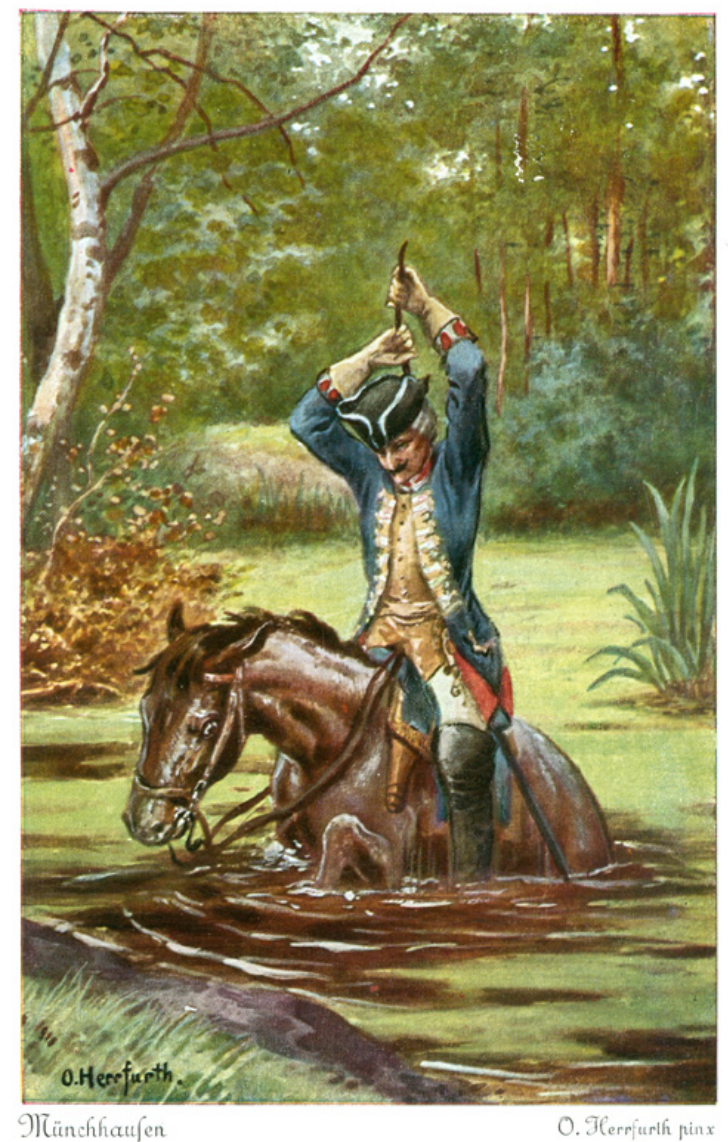

Figura 4.1: Imagen del libro de Rudolf E. Raspe, The Surprising Adventures of Baron Munchausen, donde el Barón intenta salir del pantano tirando de su cabello.

selección del L1, multiplicada por la eficiencia del L1 en relación con una muestra $S$ de fotones o jets offline:

$$
\epsilon^{B S}=\epsilon_{\mathrm{HLT} \mid \mathrm{S}}=\epsilon_{\mathrm{HLT} \mid \mathrm{L} 1} \times \epsilon_{\mathrm{L} 1 \mid \mathrm{S}}
$$

Para medir la eficiencia del HLT respecto del L1, se toma una fracción de eventos aceptados por el L1 sin una selección en el HLT (modo de operación Passed-Through) o por un trigger con requerimientos más relajados (loose) con respecto al trigger que se quiere estudiar.

Durante el Run-2, el sistema de trigger y adquisición de datos de ATLAS se configuró para ejecutar todos los triggers primarios del HLT en modo re-run. En este modo, la decisión de dicho trigger se evalúa y almacena para todos los eventos aceptados, incluso si el trigger no se había ejecutado. Esto permite la evaluación de su desempeño en datos.

La eficiencia del L1 se calcula en eventos seleccionados por triggers aleatorios o de sesgo mínimo (minimum-bias) cuyos resultados son compatibles con una eficiencia completa, y se supone igual a 1 en el presente trabajo [103].

La muestra Bootstrap se define entonces como el conjunto de eventos aceptados por, o bien el L1 con HLT en modo Passed-Through, o por un trigger con requerimientos más loose con respecto al trigger que se quiere estudiar. 


\subsection{Eficiencia del trigger de fotones}

En la búsqueda de SUSY que se realiza en esta tesis, como así también en todas las búsquedas y los cálculos de secciones eficaces de producción que incluyen fotones, conocer la eficiencia del trigger correspondiente que se utiliza para seleccionar los datos, se vuelve central. La colaboración ATLAS ha presentado resultados del trigger de electrones y fotones en varias publicaciones desde el inicio de la toma de datos en 2010, cubriendo datos de principios de 2010 [106], datos de 2011 [107] y de la toma de datos a $\sqrt{s}=13 \mathrm{TeV}$ de 2015 [108]. Como se menciona en la Sección 5.1, los datos utilizados para la búsqueda de SUSY se seleccionan usando la cadena del trigger HLT_g140_loose, donde se requiere al menos un fotón loose aislado con $E_{\mathrm{T}}>140$ $\mathrm{GeV}$. Como parte del trabajo de esta tesis, se estudian además las eficiencias de todos los triggers de fotones disponibles para todos los análisis de ATLAS durante todo el Run-2 [103].

Entre los principales triggers de fotones utilizados para las distintas búsquedas llevadas a cabo en ATLAS, se pueden mencionar a modo de ejemplo:

- g35_loose_g25_loose ${ }^{1}: \mathrm{H} \rightarrow \gamma \gamma+\mathrm{SM}$.

- 2g22_tight: Búsquedas de Higgs de baja masa + SM.

- g70_loose_xe70noL1: Búsquedas de Higgs + dark photons.

- g140_loose ${ }^{2}$ : Búsquedas de SUSY con fotones + jets/leptones.

- 2g50_loose: Búsquedas de SUSY con di-fotones.

Es importante notar aquí que para los estudios de las eficiencias, cada tramo de los triggers principales (es decir, por ejemplo, g35_loose y g25_loose para g35_loose_g25_loose) con un prescale distinto de 1, se encontraba configurado en modo re-run a fin de poder calcular sus eficiencias, como es el caso de los antes mencionados debido a su importancia en el canal de $\mathrm{H} \rightarrow \gamma \gamma$ [109, 110].

En la Tabla 4.1 se muestran los triggers usados para generar la muestra de Bootstrap (primera columna) para los distintos triggers principales a los que se les calculó su eficiencia (primera fila). Si bien esta tabla no está completa (por una cuestión de visualización), provee una idea general del proceso utilizado.

\section{Incertezas}

Para los estudio de Run-2, la incerteza sistemática para las eficiencias obtenidas con el método Bootstrap se calcula como la mitad de la diferencia entre la eficiencia obtenida en datos y la obtenida en los eventos simulados. Para los eventos simulados se utilizan muestra MC de producción de fotones directos generados con PyThia 8.186. Estas muestras incluyen los eventos de LO $\gamma+$ jet desde procesos de dispersión dura $q g \rightarrow q \gamma$ y $q \bar{q} \rightarrow g \gamma$, así como fotones directos de la fragmentación de quarks en eventos de QCD dijet. Dichos eventos simulados se consideran como fuente pura de fotones aislados mientras que las muestras utilizadas en el método de Bootstrap de datos contienen tanto fotones reales como eventos de fondo. En estos

\footnotetext{
${ }^{1}$ Este trigger se reemplazó por su versión con fotones medium durante la toma de datos de 2017 y 2018 para mantener el rate contenido.

${ }^{2}$ Durante 2015 estuvo también presente el trigger g120_loose.
} 


\begin{tabular}{|l|c|c|c|}
\hline & $\begin{array}{c}\text { g20_tight } \\
\text { g22_tight } \\
\text { g25_loose } \\
\text { g25_medium }\end{array}$ & $\begin{array}{c}\text { g35_loose } \\
\text { g35_medium } \\
\text { g50_loose }\end{array}$ & $\begin{array}{l}\text { g100_loose } \\
\text { g120_loose } \\
\text { g140_loose }\end{array}$ \\
\hline \hline HLT_g10_loose & $\mathrm{X}$ & $\mathrm{X}$ & $\mathrm{X}$ \\
HLT_g12_loose & $\mathrm{X}$ & $\mathrm{X}$ & $\mathrm{X}$ \\
HLT_g15_loose & & $\mathrm{X}$ & $\mathrm{X}$ \\
HLT_g20_loose & & & $\mathrm{X}$ \\
HLT_g60_loose & & $\mathrm{X}$ & \\
HLT_g80_loose & $\mathrm{X}$ & $\mathrm{X}$ & \\
HLT_noalg_L1EM10VH & $\mathrm{X}$ & $\mathrm{X}$ & \\
HLT_noalg_L1EM12 & $\mathrm{X}$ & $\mathrm{X}$ & \\
HLT_noalg_L1EM15 & & $\mathrm{X}$ & \\
HLT_noalg_L1EM15VH & & & \\
HLT_noalg_L1EM20VH & & & \\
\hline
\end{tabular}

Tabla 4.1: Triggers usados para generar la muestra de Bootstrap (primera columna) para los distintos triggers principales a los que se les calculó su eficiencia (primera fila).

eventos de fondo, los objetos considerados como fotones no se corresponden con un fotón real, debido a que las características de las técnicas de reconstrucción generan una tasa de identificación errónea de objetos. Este es un enfoque conservador para calcular la incerteza sistemática, ya que también incluye las discrepancias entre la simulación y los datos reales. En la Figura 4.2 se muestran las curvas de eficiencias para datos y MC (izquierda), y la semi diferencia bin a bin entre ambas curvas (derecha) para el trigger HLT_g140_loose en 2018. Las incertezas sistemáticas que se tiene son menores al $10 \%$, y son más importantes en la región de encendido del trigger o turn-on, donde el trigger no es completamente eficiente y suele no utilizarse en los análisis. Al mismo tiempo, en la región del plateau $\left(E_{\mathrm{T}}>145 \mathrm{GeV}\right)$, las incertezas son menores al $1 \%$.
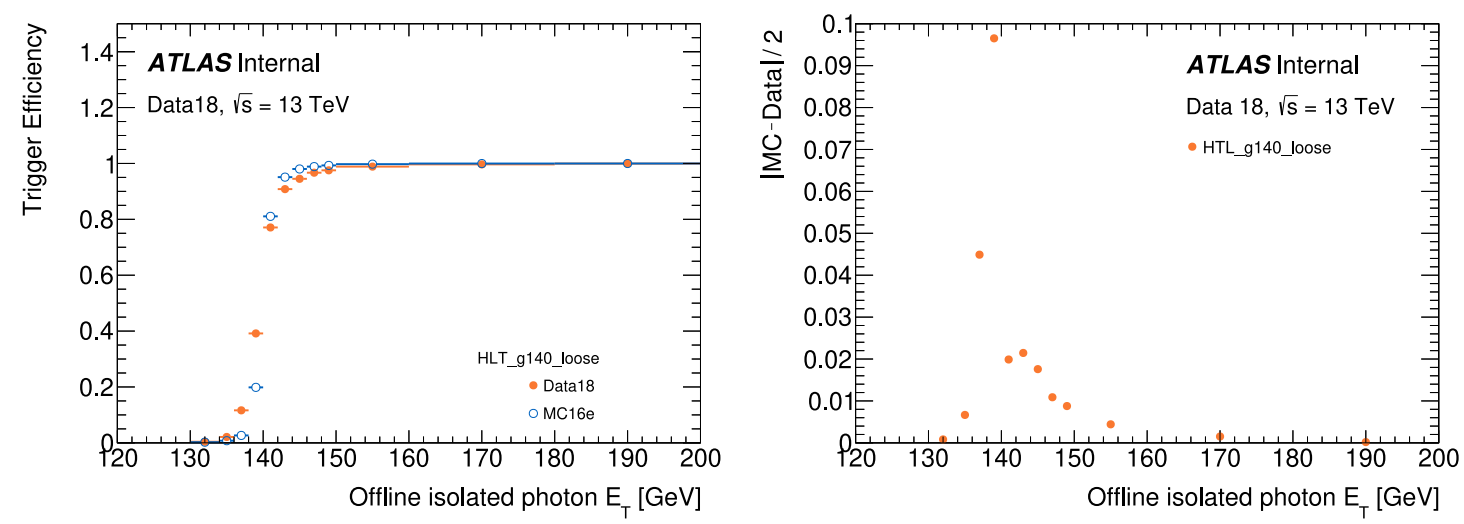

Figura 4.2: Curvas de eficiencias en datos y MC (izquierda) y la semi diferencia bin a bin entre dichas curvas (derecha) para el trigger HLT_g140_loose en 2018. Estas diferencias se utilizan como incertezas sistemáticas en la eficiencia del trigger. 


\subsubsection{Resultados de las eficiencias del trigger de fotones}

Las eficiencias en función de la energía transversa $\left(E_{\mathrm{T}}\right)$ y el $\eta$ de los fotones, y del número medio de interacciones por bunch crossing $(\langle\mu\rangle)$, para los triggers g25_loose (o g25_medium), g35_loose (o g35_medium) y g140_loose se muestran en las Figuras 4.3 para las tomas de datos de 2015 y 2016, y en 4.4 para 2017 y 2018. Se calculan con respecto a fotones offline que pasan el criterio de identificación tight y requisitos de aislamiento calorimétrico (WP: FixedCutTightCaloOnly, véase sección 3.1.3).

Los candidatos a fotones offline cercanos a la región de transición endcap-barrel $1.37<|\eta|<1.52$ no se consideran para calcular la eficiencia del trigger debido a la baja instrumentación de esta región (véase 2.2.4).

La forma teórica de una curva de eficiencia en función de $E_{\mathrm{T}}$ es una función escalón perfecta. Para eventos con un fotón de $E_{\mathrm{T}}$ menor al umbral del trigger la eficiencia es cero, mientras que para aquellos con un fotón cuyo $E_{\mathrm{T}}$ supere el umbral es del $100 \%$. Sin embargo, como se puede ver en (a) y (b) de las Figuras 4.3 y 4.4, la antes mencionada turn-on no crece de manera tan abrupta, debido a que los objetos considerados para este estudio son previamente seleccionados a nivel online con las respectivas incertezas involucradas. De todas formas, queda claro el excelente rendimiento del trigger, al notar que la eficiencia alcanza el plateau (>99\%) para fotones con $E_{\mathrm{T}}$ al menos $5 \mathrm{GeV}$ por encima del umbral del trigger.

Para las eficiencias en función de $\eta \mathrm{y}\langle\mu\rangle$, se consideran aquellos eventos con al menos un fotón de $E_{\mathrm{T}}>5 \mathrm{GeV}$ al umbral del trigger, de manera de estudiar la dependencia con estas variables en la región energética de interés para los análisis. De (c) y (d) se observa que no existe una dependencia con $\eta$. Por su parte, (e) y (f) muestran que las eficiencias de los triggers de fotones se mantuvieron estables (cercanas al $100 \%$ ) con el aumento del número de protones colisionantes y de la luminosidad instantánea proporcionada por el LHC durante los años de toma de datos del Run-2.

Por lo tanto, se puede concluir que los triggers de fotones loose y medium son altamente eficientes para fotones offline, tight, aislados y con un $p_{\mathrm{T}}$ de al menos 5 $\mathrm{GeV}$ por encima del umbral del trigger.

Por último, en la Figura 4.5 se muestra la eficiencia en el plateau para el trigger g140_loose, tanto para datos de 2018 como para MC, a fin de obtener el valor de la eficiencia en el rango de $E_{\mathrm{T}}>145 \mathrm{GeV}$, al que se referirá luego en el Capítulo 5 .

Las eficiencias que se obtienen, independientemente del año son:

- Data: $99.251 \pm 0.031 \%$

- MC: $99.862 \pm 0.001 \%$

donde las incertezas mostradas son sólo estadísticas.

\subsection{Método de estimación de fondo para cálculos de eficiencias}

Debido a que jets erróneamente identificados como fotones pueden disparar los Bootstrap triggers, existe una contaminación de fondo en la muestra Bootstrap por lo que, a diferencia de una muestra de fotones provenientes del decaimiento radiativo 


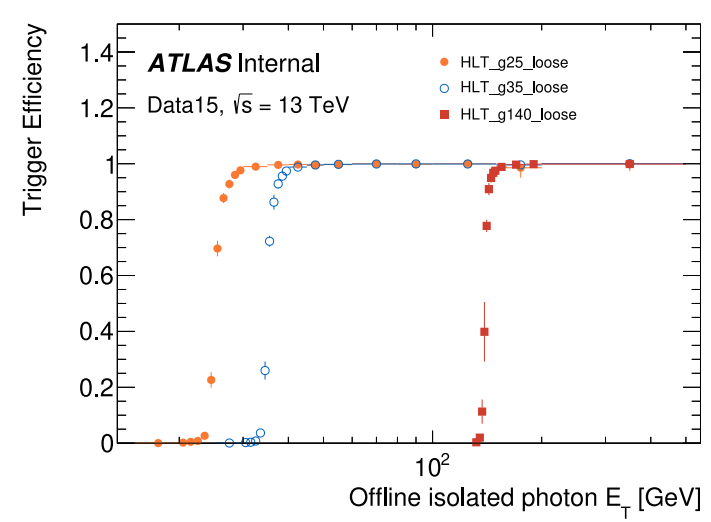

(a) 2015: $E_{\mathrm{T}}$

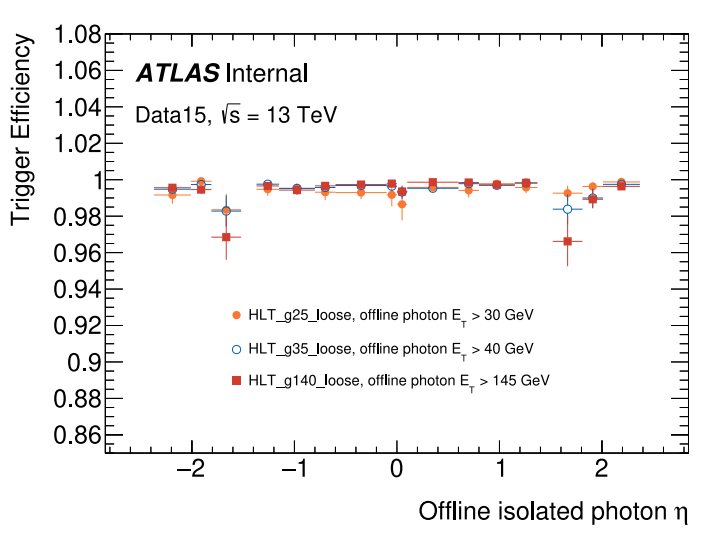

(c) 2015: $\eta$

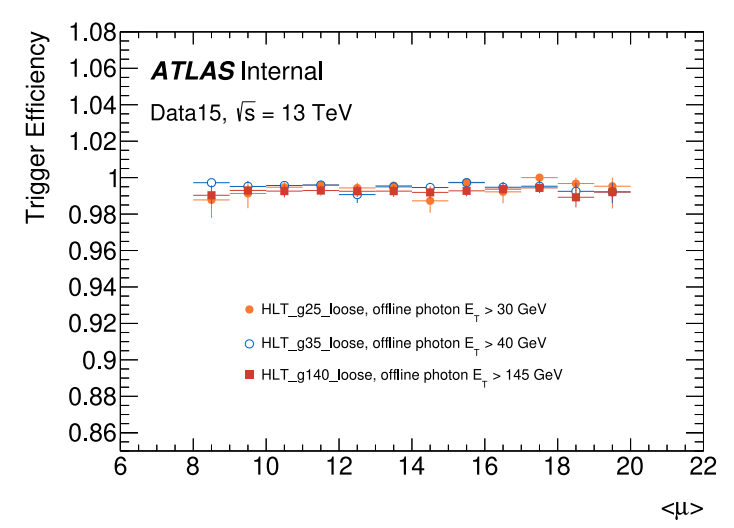

(e) $2015:\langle\mu\rangle$

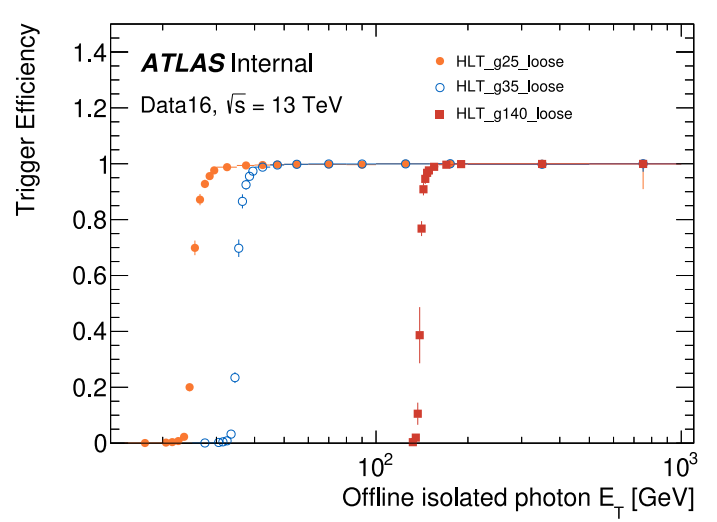

(b) 2016: $E_{\mathrm{T}}$

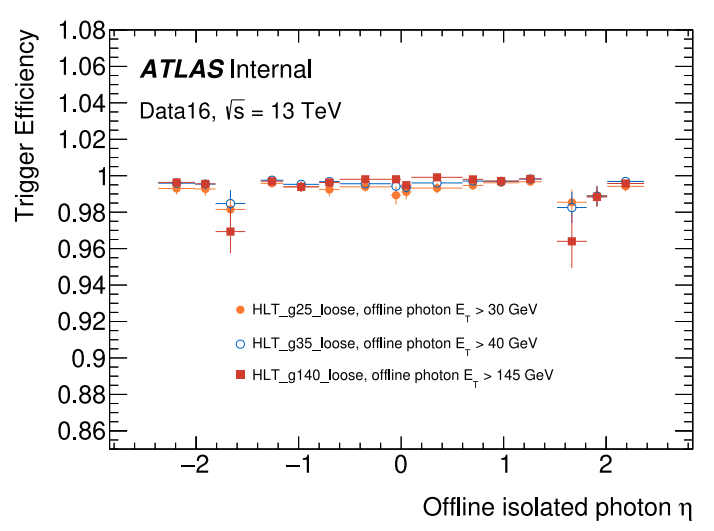

(d) 2016: $\eta$

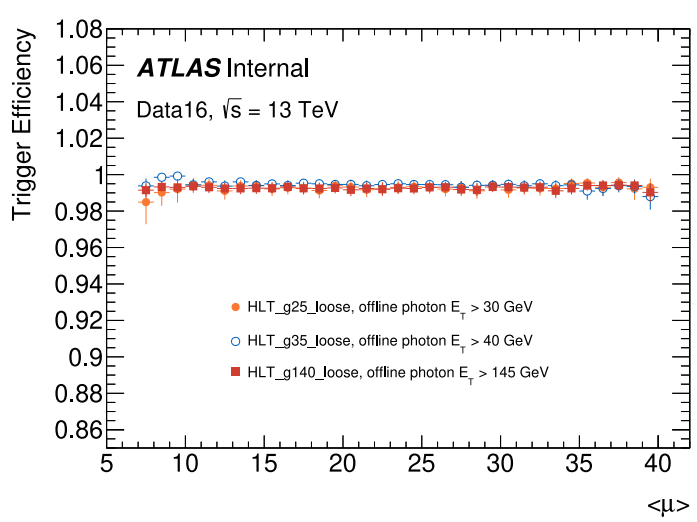

(f) 2016: $\langle\mu\rangle$

Figura 4.3: Eficiencias de los triggers primarios de fotones en función de $E_{\mathrm{T}} \eta \mathrm{y}\langle\mu\rangle$ para todos los datos de 2015 (izquierda) y 2016 (derecha). 


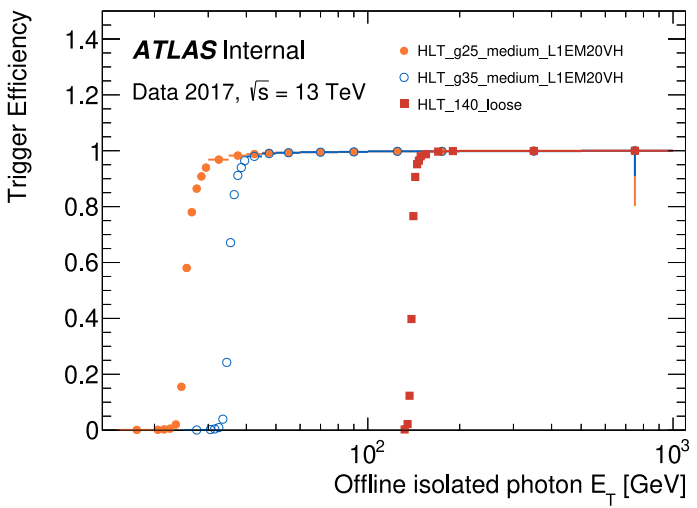

(a) 2017: $E_{\mathrm{T}}$

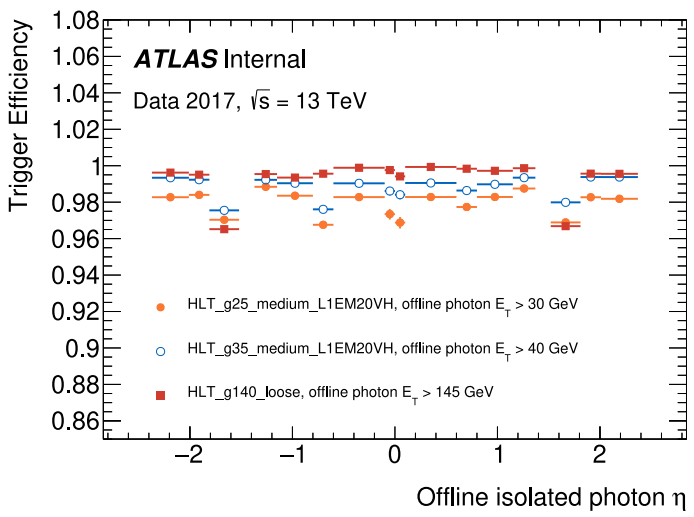

(c) $2017: \eta$

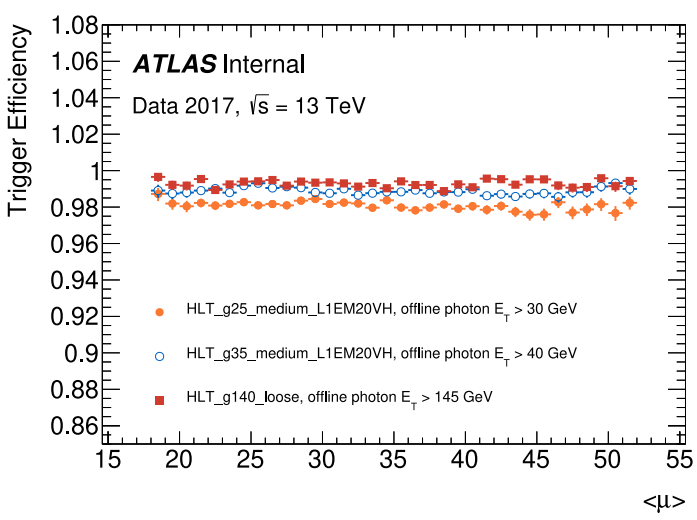

(e) $2017:\langle\mu\rangle$

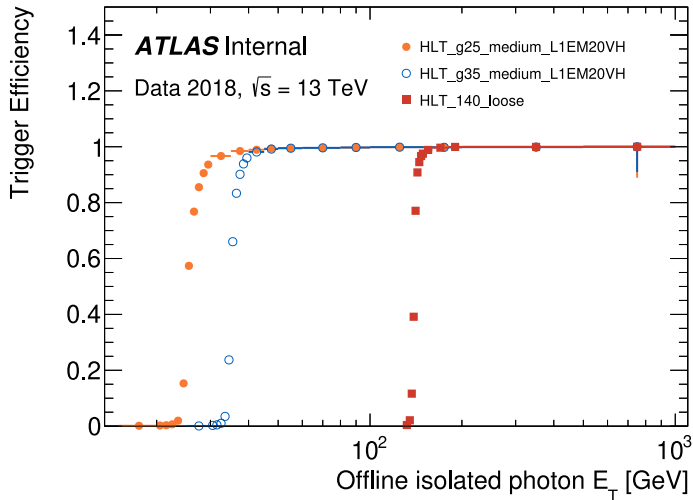

(b) 2018: $E_{\mathrm{T}}$

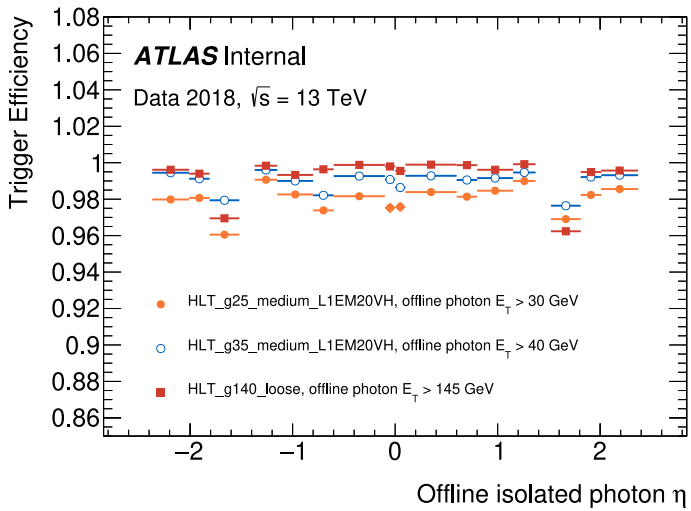

(d) 2018: $\eta$

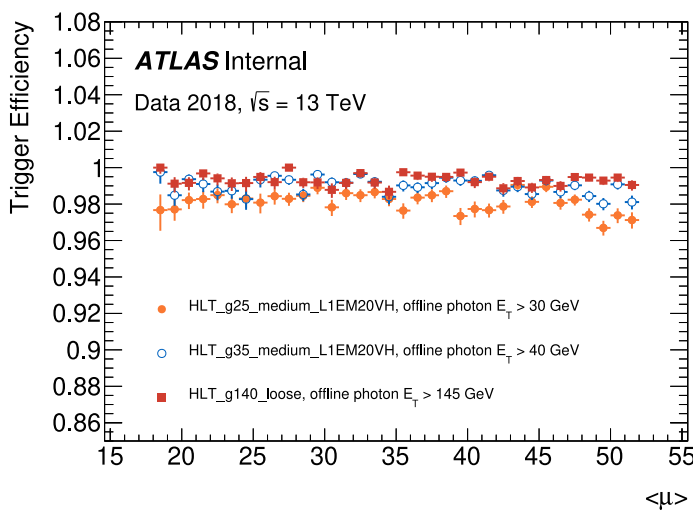

(f) 2018: $\langle\mu\rangle$

Figura 4.4: Eficiencias de los triggers primarios de fotones en función de $E_{\mathrm{T}} \eta \mathrm{y}\langle\mu\rangle$ para todos los datos de 2017 (izquierda) y 2018 (derecha). 


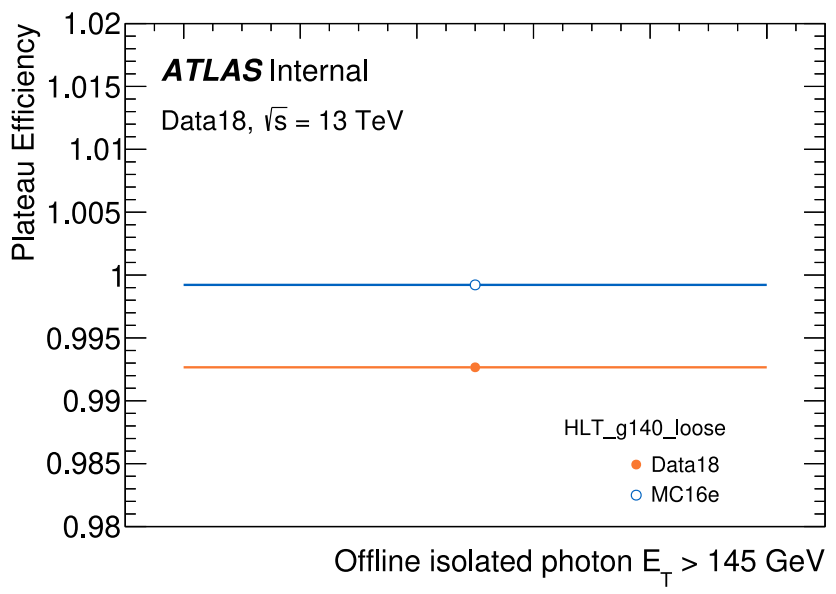

Figura 4.5: Eficiencias en el plateau para datos 2018 y MC del trigger g140_loose para $E_{\mathrm{T}}>145 \mathrm{GeV}$.

del $Z$ a dos leptones, no es una fuente de fotones pura. Hay una fracción de objetos que satisfacen los requisitos de aislamiento y de identificación tight del fotón, sin ser fotones reales, incluidos en la muestra Bootstrap. El método de conteo bidimensional que se detalla a continuación, provee una estimación de este fondo.

La eficiencia de los triggers en los candidatos a fotones de la muestra Bootstrap es entonces una combinación de la eficiencia de los triggers en fotones reales (referidos como señal) y en fotones falsos que satisfacen la identificación del fotón (referidos como fondo). Esto se describe en la Ecuación 4.2 donde $\epsilon$ representa la eficiencia del trigger, $N_{P}$ y $N_{T}$ son el número de candidatos a fotones que pasan la selección del trigger y el número total de candidatos, respectivamente. Asimismo, los superíndices $B S, s$ y $b$ representan las muestras de Bootstrap, de señal y de fondo, respectivamente.

$$
\epsilon^{B S}=\frac{N_{P}}{N_{T}}=\frac{N_{P}^{s}+N_{P}^{b}}{N_{T}}=\frac{\epsilon^{s} \cdot N_{T}^{s}+\epsilon^{b} \cdot N_{T}^{b}}{N_{T}}
$$

La idea detrás de la sustracción de fondo consiste en obtener la eficiencia del trigger en la muestra de señal $\epsilon^{s}$ como una función de la eficiencia del trigger en el fondo $\epsilon^{b}$ y de la pureza de la muestra Bootstrap (que se define más adelante). Siendo $N_{T}=N_{T}^{s}+N_{T}^{b}$ y P la pureza, se tiene que $N_{T}^{s}=P \cdot N_{P}$ y $N_{T}^{b}=(1-P) \cdot N_{P}$, con los que se puede obtener la expresión 4.3 para la eficiencia de señal.

$$
\epsilon^{S}=\frac{1}{P}\left[\epsilon^{B S}-\epsilon^{b}(1-P)\right]
$$

La pureza de la muestra Bootstrap se obtiene mediante el método data-driven $\mathrm{ABCD}[111,112]$ que consiste en dividir la muestra de fotones en cuatro categorías. Para definir las categorías se utiliza la variable de aislamiento $E_{\mathrm{T}}^{\text {iso }}$ (definida en la Sección 3.1.3), y la identificación tight o non-tight de los objetos.

La identificación non-tight requiere que los candidatos pasen todos los cortes de selección tight de fotones excepto, al menos, uno de los requerimientos sobre las siguientes variables, $w_{s, 3}, F_{\text {side }}, E_{\text {ratio }}$ y $\Delta E$, que se definieron en el Capítulo 3. De 
esta manera se obtiene suficiente estadística en la muestra de fondo, manteniendo una débil correlación con la variable de aislamiento.

Las cuatro categorías se muestran en la Tabla 4.2, donde la región A es la región de la señal, y B, C y D son las regiones de control o fondo conformadas por objetos no aislados o que fallan la selección tight en alguna de sus variables de identificación. La distribución de eventos en estas regiones se muestran en la Figura 4.6.

\begin{tabular}{l|c|c} 
& $E_{\mathrm{T}}^{\text {iso }}<0.022 \times E_{T}^{\gamma}+2.45 \mathrm{GeV}$ & $E_{\mathrm{T}}^{\text {iso }}>0.022 \times E_{T}^{\gamma}+5.45 \mathrm{GeV}$ \\
\hline Fotón Tight & $\mathrm{A}$ & $\mathrm{B}$ \\
Fotón Non-tight & $\mathrm{C}$ & $\mathrm{D}$
\end{tabular}

Tabla 4.2: Las cuatro categorías de los candidatos a fotones usadas para estimar la pureza de la muestra de fotones.

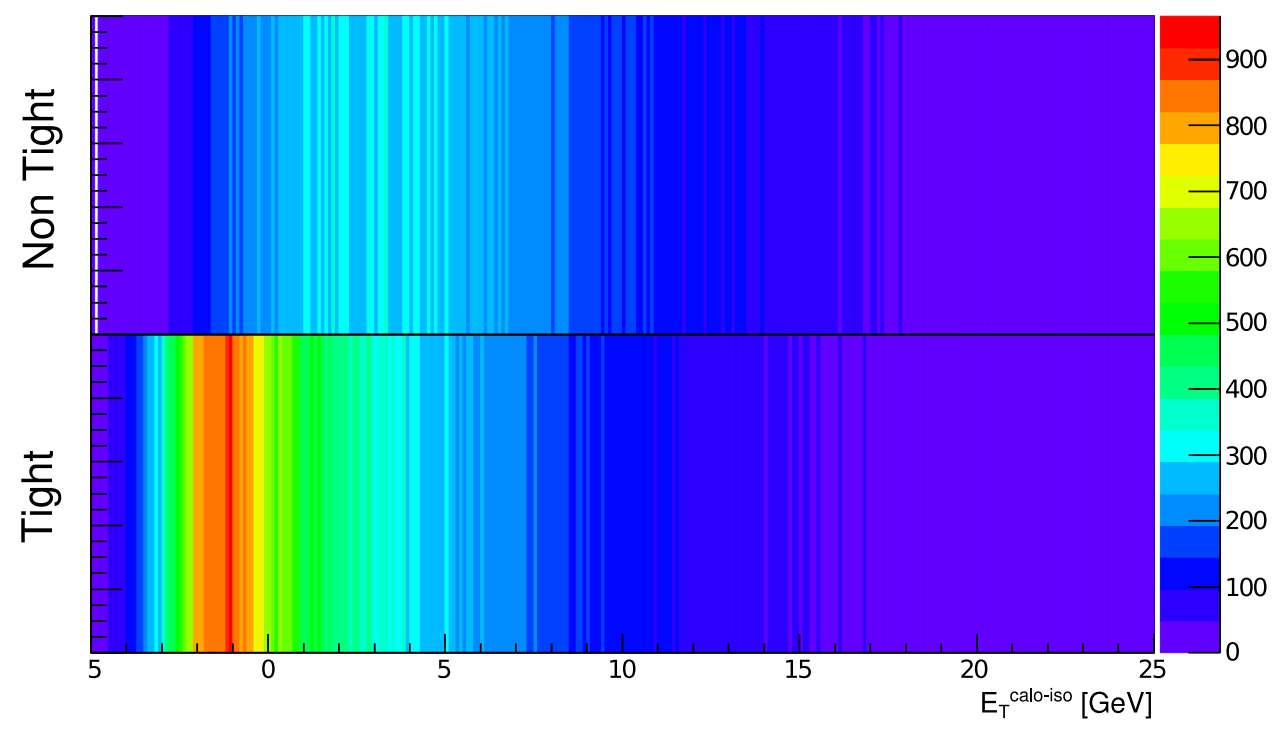

Figura 4.6: Distribución de eventos en el plano Identificación-Aislamiento para los datos de 2015 que pertenecen a la muestra Bootstrap.

Si se supone que no hay correlación entre el aislamiento y la identificación del fotón, e ignorando la filtración de señal en las regiones de fondo, la pureza se puede obtener como se indica en la Ecuación 4.4, donde $N_{A}^{s i g}$ es la señal total en la región $A$, y $N_{A}, N_{B}, N_{C}$ y $N_{D}$ son el número de eventos en las regiones $\mathrm{A}, \mathrm{B}, \mathrm{C}$ y $\mathrm{D}$, respectivamente.

$$
\text { Pureza }=\frac{N_{A}^{s i g}}{N_{A}}=1-\frac{N_{B} N_{C}}{N_{A} N_{D}}
$$

Si se tiene en cuenta la filtración de señal en las regiones de fondo, entonces el número de eventos en estas regiones se convierten en $N_{\lambda}-k_{\lambda} N_{A}^{\text {sig }}$ para $\lambda=$ $\{\mathrm{B}, \mathrm{C}, \mathrm{D}\}$, donde $k_{\lambda}$ es la fracción de la señal filtrada a la región de control $\lambda$. La señal filtrada se determina a partir de cómo los fotones reales pueblan estas regiones 
(para lo que se usan simulaciones MC). Con estas correcciones la expresión para la pureza se vuelve cuadrática en $N_{A}^{s i g}$, como puede verse en la Ecuación 4.5.

$$
N_{A}^{s i g}=N_{A}-\left[\left(N_{B}-k_{B} N_{A}^{s i g}\right) \frac{N_{C}-k_{C} N_{A}^{s i g}}{N_{D}-k_{D} N_{A}^{s i g}}\right] .
$$

\section{Incertezas sistemáticas de la pureza}

La incerteza sistemática del método Bootstrap con sustracción de fondo usando el método ABCD para calcular la pureza de la muestra, proviene precisamente de la medición de la pureza. Dicha medición tiene dos fuentes principales de incerteza. Por un lado, la estimación de la filtración de señal en las regiones de fondo del método ABCD y, por otro lado, la elección arbitraria de los cortes a invertir utilizados para definir las regiones non-tight.

Para la primera fuente, la incerteza se obtiene a partir de la diferencia entre la filtración de señal a fondo calculada usando fotones de señal de simulaciones MC y fotones de datos en eventos del decaimiento radiativo $Z$.

Para la segunda fuente de incerteza, se elige arbitrariamente un subconjunto en base a tres de las cuatro variables utilizadas para definir los fotones non-tight, generando una región diferente (y aún así válida) dominada por fondo. En la próxima sección se muestran los resultados teniendo en cuenta estas incertezas sistemáticas.

\subsubsection{Resultados de las eficiencias con sustracción de fondo para datos de 2015}

La pureza (Ec. 4.4) de la muestra Bootstrap para el trigger HLT_g50_1oose, con y sin correcciones se muestra en la Figura 4.7 en función de $E_{\mathrm{T}}$. Se concluye de dicha figura que los efectos introducidos por la correlación entre la ID y el aislamiento, sumados a los de la filtración de señal en las regiones de fondo, no afectan sustancialmente a la pureza de la muestra Bootstrap, que es del $\sim 80 \%$ para $E_{\mathrm{T}}>5 \mathrm{GeV}$ por encima del requerimiento del trigger.

En la Figura 4.8 se muestra la eficiencia del trigger HLT_g50_loose en función de $E_{\mathrm{T}}$ para una muestra de fotones reales de $\mathrm{MC}$, y para datos utilizando el método de Bootstrap sin sustracción de fondo (BS eff) y con sustracción de fondo con el método ABCD (Signal eff). Se calcula con respecto a fotones offline que pasan el criterio de identificación tight y requisitos de aislamiento FixedCutTightCaloOnly, sin incluir la región de transición endcap-barrel.

En las figuras anteriores, asociadas al presente método, se observan los valores de las incertezas sistemáticas intrínsecas al método, siendo éstas muy superiores a las obtenidas en el método de Bootstrap sin sustracción de fondo. En base a estos resultados preliminares, se decidió utilizar el método de Bootstrap sin sustracción de fondo y asignarle una incerteza sistemática en base a las diferencias entre los valores encontrados en datos y en las simulaciones, como se detalló en la sección anterior. 


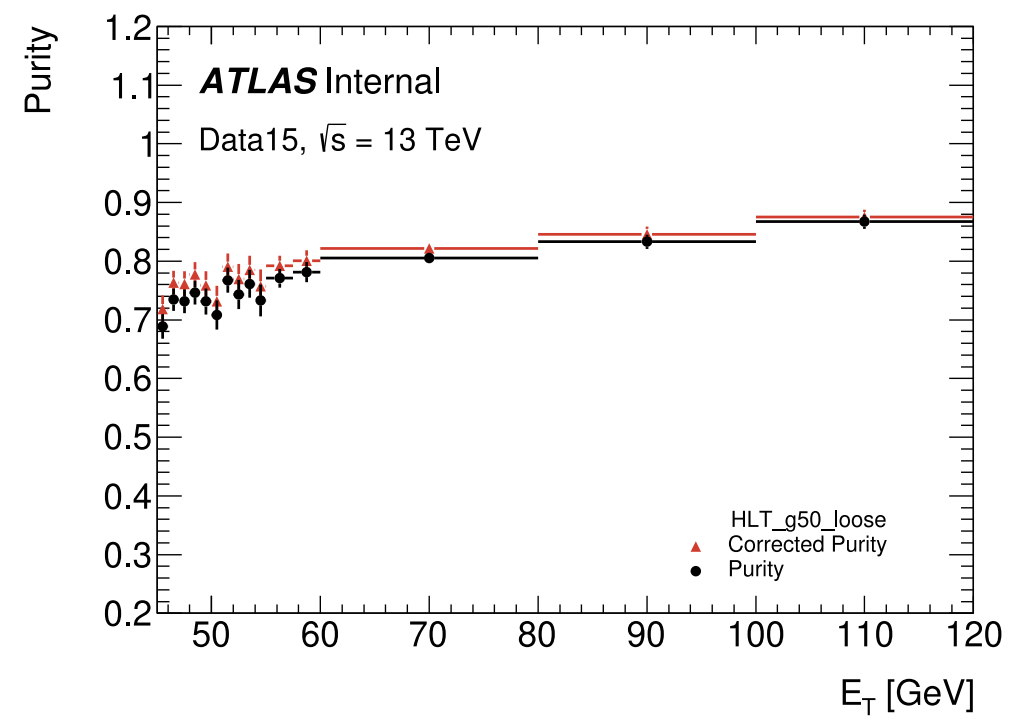

Figura 4.7: Pureza de la muestra Bootstrap para el trigger HLT_g50_1oose en función de $E_{\mathrm{T}}$ para todos los datos de 2015 .

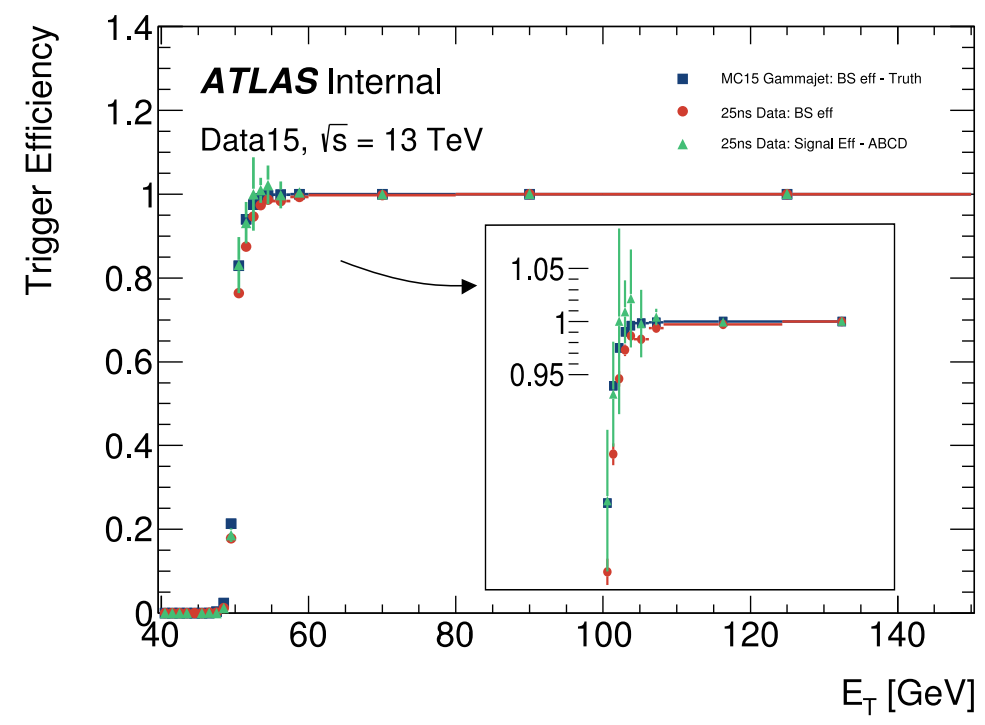

Figura 4.8: Eficiencias del trigger HLT_g50_loose en función de $E_{\mathrm{T}}$ para todos los datos de 2015 y MC, con y sin sustracción de fondo. Las incertezas son estadísticas para todos los puntos, a excepción de los puntos representados como triángulos, donde se incluyen además las incertezas sistemáticas del método ABCD. 


\subsection{Eficiencia del trigger de multijets}

Las cadenas de trigger con múltiples jets se utilizan para seleccionar los eventos, por ejemplo, en la búsqueda de SUSY con multijets (hasta más de 12) y $E_{\mathrm{T}}^{\text {miss }}$ en el estado final $[113]^{3}$. Este análisis utiliza básicamente dos requerimientos distintos en el $p_{\mathrm{T}}$ de los jets (50 y $80 \mathrm{GeV}$ ), para definir las distintas regiones de señal, por lo que los diferentes triggers aquí analizados tienen un requerimiento online menor a uno $\mathrm{u}$ otro de dichos valores. Sin embargo, todas las cadenas comienzan con la selección del primer nivel del trigger (L1, basado en hardware), donde se requiere que los eventos contengan cuatro jets con $p_{\mathrm{T}}>15 \mathrm{GeV}$ (L1_4J15) y, en algunos casos, con una restricción adicional de $|\eta|<2.5$ (L1_4J150ETA25).

Los eventos seleccionados por el L1 pasan al HLT, donde la selección del jet se realiza sobre aquellos jets reconstruidos a partir de clusters topológicos tridimensionales de energía medidos en las celdas del calorímetro, y calibrados de forma similar a los jets offline descriptos en la Sección 3. Para este análisis, se combinan varios triggers diferentes para seleccionar eventos. Debido al aumento de la luminosidad del LHC y a la mejora de los métodos de reconstrucción en el HLT, las definiciones de los triggers evolucionaron a lo largo del Run-2.

En particular, en 2017-2018 se implementaron algunas cadenas de trigger utilizando Global Sequential Calibration [114], usando trazas de la reconstrucción del b-tagging del HLT para reducir las diferencias de respuesta entre los jets iniciados por quarks y gluones.

Los requisitos de selección y los correspondientes períodos de datos para cada trigger utilizado en el análisis se indican en la Tabla 4.3. Las eficiencias se calcularon con el método de Bootstrap, definido al inicio del capítulo, utilizando las derivaciones SUSY4, descriptas en la Sección 2.4. En este caso, la muestra de Bootstrap se consigue a partir de eventos disparados por el trigger de muones HLT_mu26_medium (que selecciona muones con un $p_{\mathrm{T}}$ de al menos $26 \mathrm{GeV}$ ), generando una muestra totalmente libre de sesgo con cualquiera de las selecciones de los triggers de interés.

Las eficiencias para los principales triggers con 5, 6 y 7 jets, sin pre-escaleo, se muestran en la Figura 4.9 para EMTopo y en la Figura 4.10 para PFlow, tanto para datos como para una muestra de $t \bar{t} \mathrm{MC}$, en función del momento transversal del último jet sobre el que se aplica la selección. En particular para el caso de 5 jets en 2015 y 2016, se decidió utilizar un OR lógico entre los triggers HLT_5j60 (sin preescaleo para 2015) y HLT_5j65_0eta240_L14J15 (sin pre-escaleo para 2016), para incrementar la estadística disponible.

\subsubsection{Comparación de las eficiencias del trigger de múltiples jets versus EMTopo y PFlow jets}

Los jets reconstruidos mediante técnicas de flujo de partículas (PFlow) demuestran una mayor estabilidad frente al pile-up y una mejor resolución energética a bajo $p_{\mathrm{T}}$ con menores incertezas, lo que motiva su uso para algunos estudios de Run-2 y para todos los análisis futuros que se basen en datos del Run-3. Los algoritmos del trigger, sin embargo, utilizaron definiciones de jets basadas puramente en el calorí-

\footnotetext{
${ }^{3}$ La participación en este trabajo y parte de los estudios presentados en esta tesis (en particular la presente sección y el Capítulo 7), se iniciaron producto de una beca del gobierno suizo para una estadía doctoral de un año en la Universidad de Ginebra
} 


\begin{tabular}{l|ccc}
\hline Trigger & Selección de Jets & Calibración de Jets & Período de Datos \\
\hline 5j60 & $p_{\mathrm{T}}>70 \mathrm{GeV}$ & MC JES & 2015 \\
5j65_0eta240_L14J15 & $p_{\mathrm{T}}>65 \mathrm{GeV}, \eta<2.4$ & MC JES & 2016 \\
5j50_gsc70_0eta240_L14J150ETA25 & $p_{\mathrm{T}}>70 \mathrm{GeV}, \eta<2.4$ & Track GSC & 2017 \\
5j50_gsc70_0eta240_L14J15 & $p_{\mathrm{T}}>70 \mathrm{GeV}, \eta<2.4$ & Track GSC & 2018 \\
\hline 6j45_0eta240 & $p_{\mathrm{T}}>45 \mathrm{GeV}, \eta<2.4$ & MC JES & $2015-2016$ \\
$7 \mathrm{j} 25$ _gsc45_L14J150ETA25 & $p_{\mathrm{T}}>45 \mathrm{GeV}$ & Track GSC & 2017 \\
$7 \mathrm{j} 35$ _ssc45_L14J15 & $p_{\mathrm{T}}>45 \mathrm{GeV} \mathrm{GeV}$ & Track GSC & 2018 \\
\hline
\end{tabular}

Tabla 4.3: Triggers sin pre-escaleo, utilizados para colectar los datos para el análisis de Multijets entre 2015 y 2018, indicando los métodos de calibración utilizados. Si no se indica explícitamente, la selección a nivel hardware se realizó con L1_4J15. "MC JES" indica que el trigger se calibró con sólo un factor de escala global para restaurar la respuesta media frente a los particle-level jets. "Track GSC" implica que se aplicó la cadena de calibración completa, incluyendo el paso del GSC basado en las trazas, en cuyo caso el primer umbral de trigger especificado indica los cortes de preselección aplicados antes de la calibración con las trazas. Los nombres de los triggers de las cadenas de GSC se han truncado para eliminar el prefijo "HLT_" y los sufijos "_boffperf_split", que indican que las trazas con b-tagging se utilizaron para GSC sin ejecutar el b-tagging.

metro para todo el período de toma de datos del Run-2, lo cual genera ineficiencias al comparar con jets reconstruidos con PFlow a nivel offline, y dependiendo de los estudios realizados, se puede necesitar requerir tenerlas en cuenta.

Como se muestra en la Figura 4.11, los triggers de multijets saturan a más altos valores del $p_{\mathrm{T}}$ del jet para PFlow offline, con respecto a los jets del calorímetro. Esta diferencia es entendida considerando que se espera que las curvas de eficiencia de los triggers alcancen los valores más altos de eficiencias a valores de $p_{\mathrm{T}}$ más cercanos al valor del umbral, cuando la reconstrucción offline y online sean más similares entre sí. Las eficiencias como función de $\eta$ se muestran en la Figura 4.12, donde se observa la diferencia antes mencionada entre las colecciones de jets, y la no dependencia de la eficiencia con esta variable. De manera análoga a lo realizado para fotones, las eficiencias en función de $\eta$ utilizan los objetos correspondientes que posean un $p_{\mathrm{T}}$ mayor a $5 \mathrm{GeV}$ del umbral del trigger.

\subsection{Propuesta de nuevo trigger combinado de mul- tijets y un fotón}

A fines de 2017 se logró incluir una nueva cadena de trigger en el menú, ideada para mejorar la ganancia de señal en modelos de Stealth SUSY como los que se describen en el Capítulo 7. Este nuevo trigger combina el requerimiento de un fotón loose con $p_{\mathrm{T}}>45 \mathrm{GeV}$ y seis jets con igual requerimiento en $p_{\mathrm{T}}$, dentro del rango $0<$ $|\eta|<2.4$ : HLT_g45_loose_6j45_0eta240. En esta sección simplemente se muestran las eficiencias obtenidas para datos de 2018, y se refiere al capítulo mencionado para mayor detalle.

Este trigger fue implementado sólo para la toma de datos de 2018, y en la Figura 4.13 se muestran las eficiencias como función de $\eta$ y $p_{\mathrm{T}}$ en datos, para jets EMTopo y PFlow, calculadas en función del $p_{\mathrm{T}}$ del fotón leading de manera análoga a las eficiencias calculadas en la sección anterior. Se puede ver una alta eficiencia, cercana 


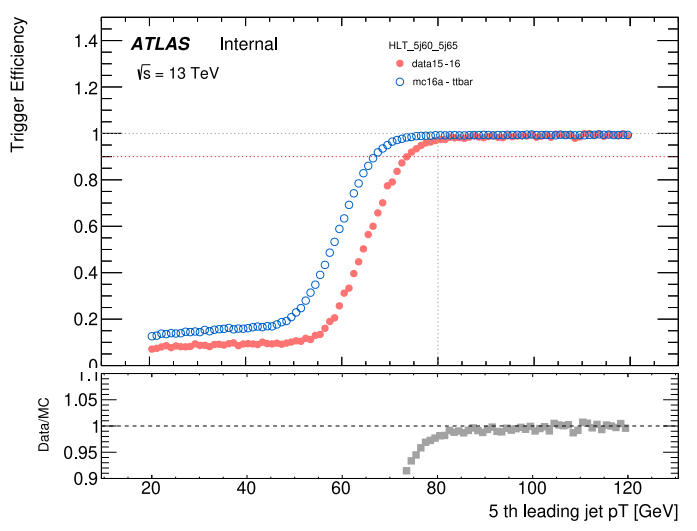

(a) $2015-2016: 5 j 60|| 5 j 65$

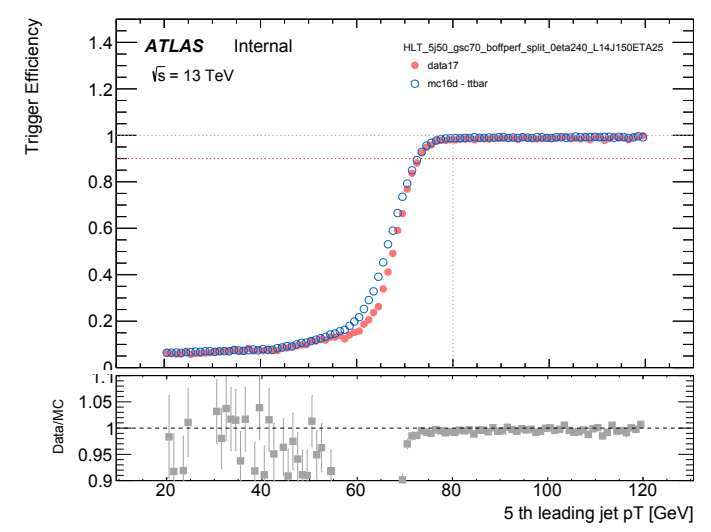

(c) 2017: 5j50_gsc70

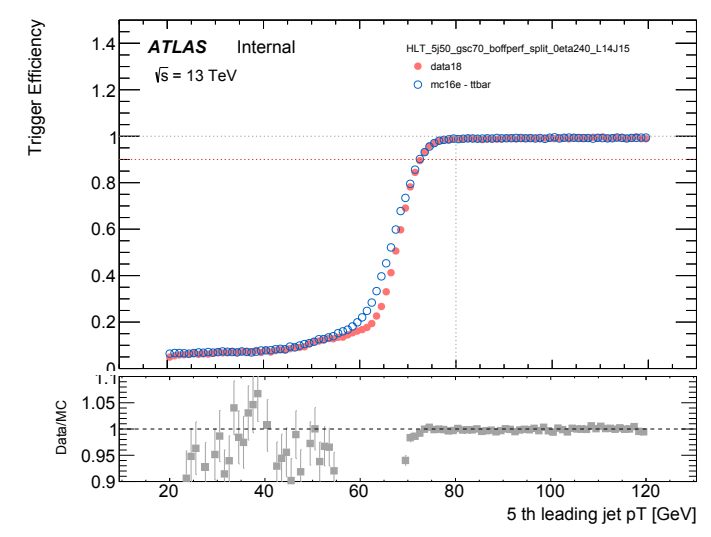

(e) 2018: 5j50_gsc70

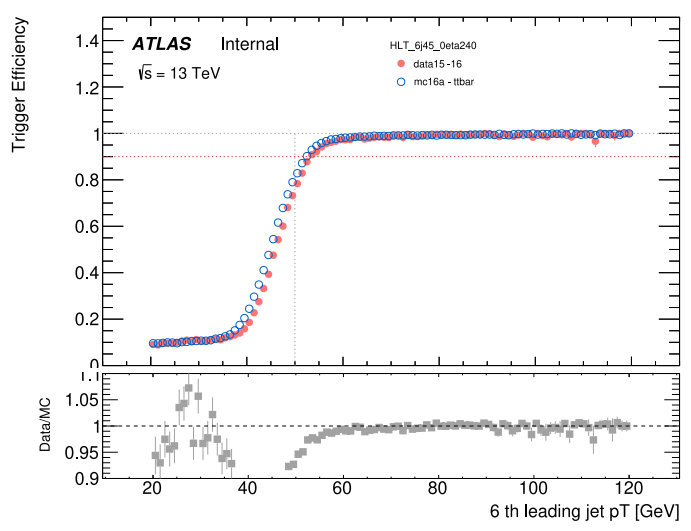

(b) 2015-2016: 6j45

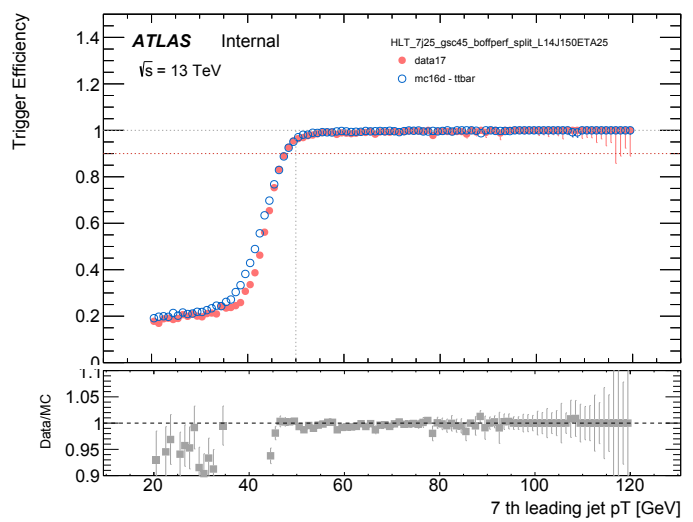

(d) $2017: 7 j 25 \_g s c 45$

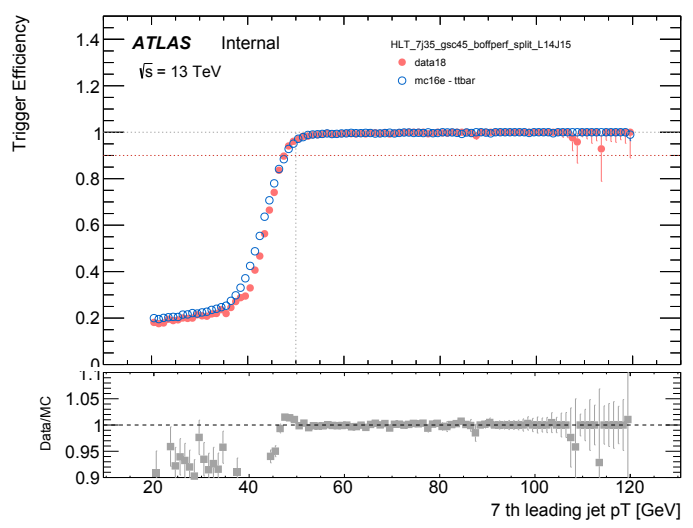

(f) 2018: 7j35_gsc45

Figura 4.9: Eficiencias, en datos y MC, de los triggers con $5 \mathrm{j}, 6 \mathrm{j}, 7 \mathrm{j}$ y no preescaleados, utilizados para colectar los datos para el análisis, en función del momento transversal del último jet sobre el que se aplica la selección, para los jets EMTopo.

al $98 \%$, alcanzando el plateau a los $10 \mathrm{GeV}$ más allá del requerimiento del trigger y sin dependencia con $\eta$, lo que lo vuelve un trigger ideal para la búsqueda de Stealth SUSY, como se discute en el Capítulo 7. 


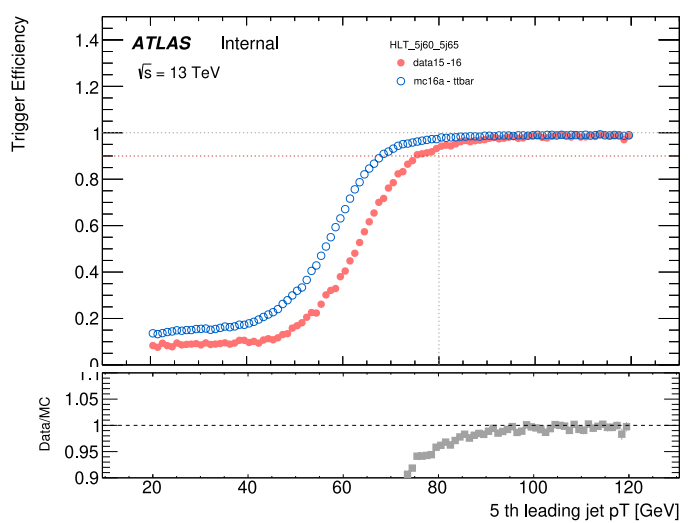

(a) $2015-2016: 5 j 60|| 5 j 65$

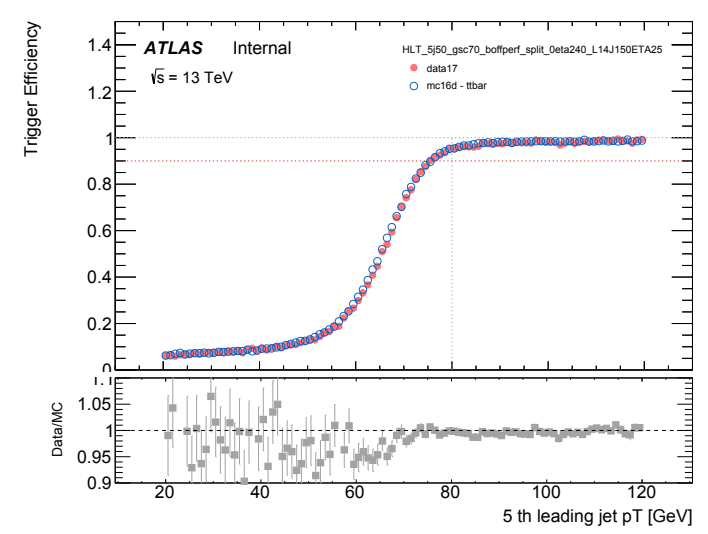

(c) 2017: 5j50_gsc70

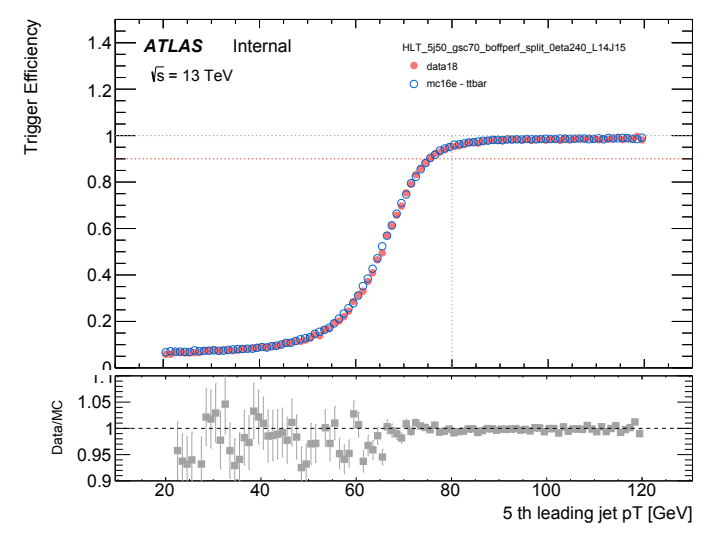

(e) 2018: 5j50_gsc70

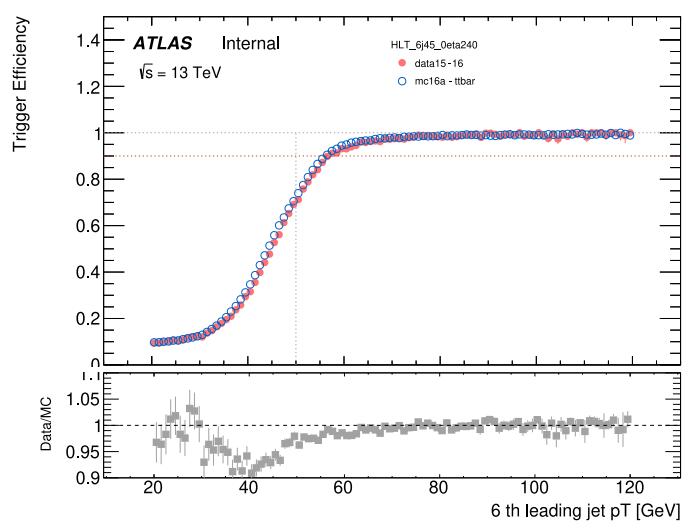

(b) 2015-2016: 6j45

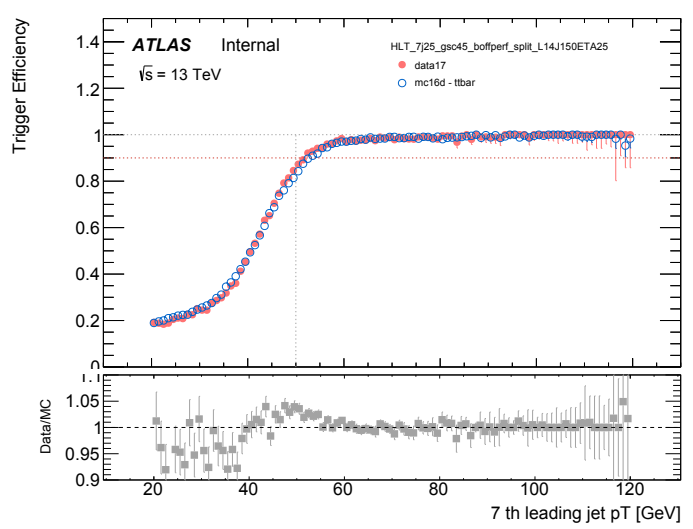

(d) 2017: 7j25_gsc45

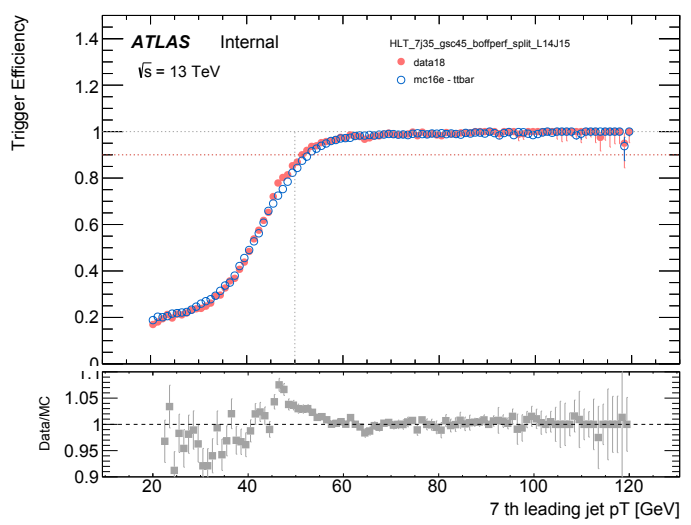

(f) $2018: 7 j 35 \_g s c 45$

Figura 4.10: Eficiencias, en datos y MC, de los triggers con $5 \mathrm{j}, 6 \mathrm{j}, 7 \mathrm{j}$ y no preescaleados, utilizados para colectar los datos para el análisis, en función del momento transversal del último jet sobre el que se aplica la selección, para los jets PFlow. 


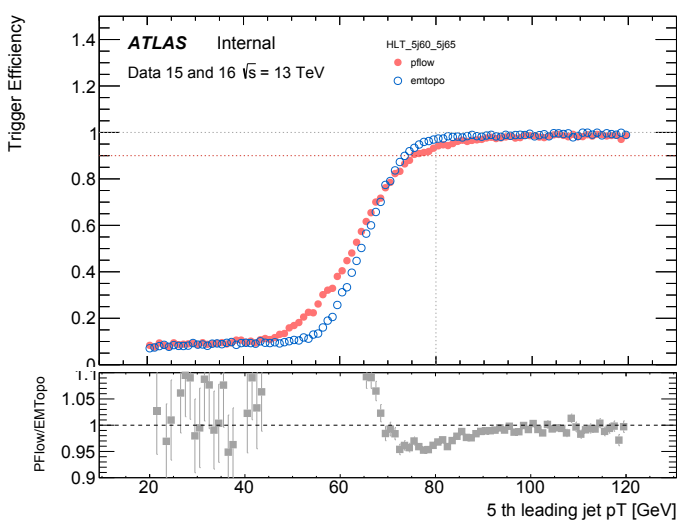

(a) $2015-2016: 5 j 60|| 5 j 65$

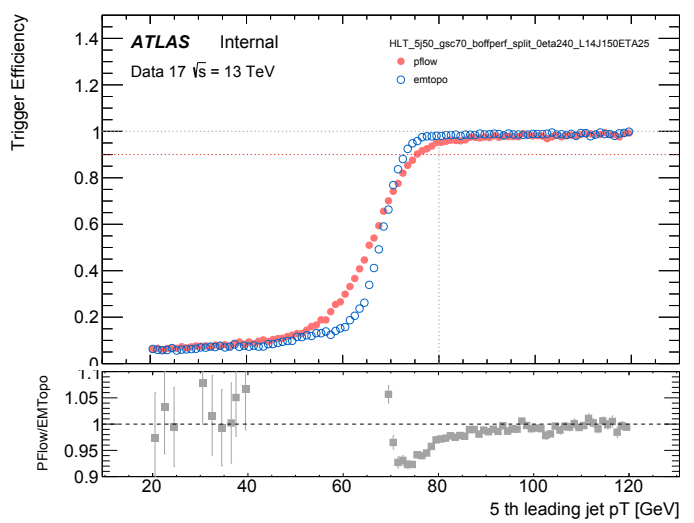

(c) 2017: 5j50_gsc70

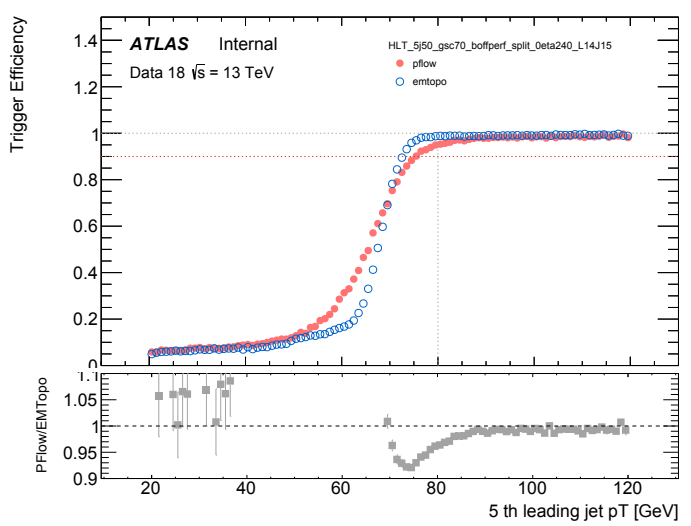

(e) 2018: 5j50_gsc70

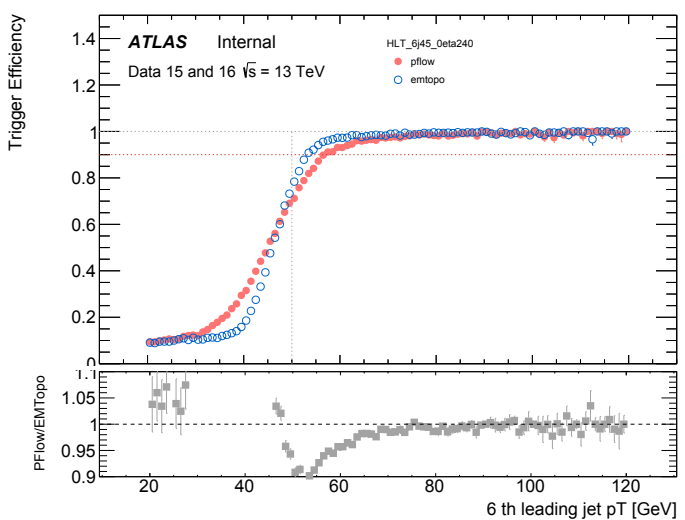

(b) $2015-2016: 6 j 45$

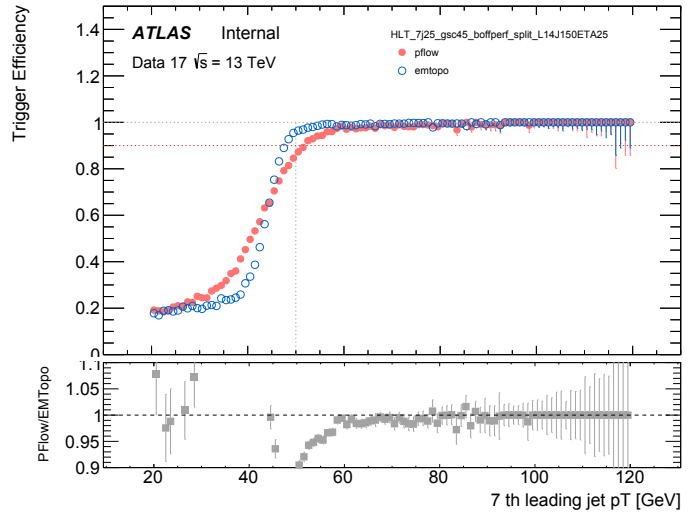

(d) 2017: 7j25_gsc45

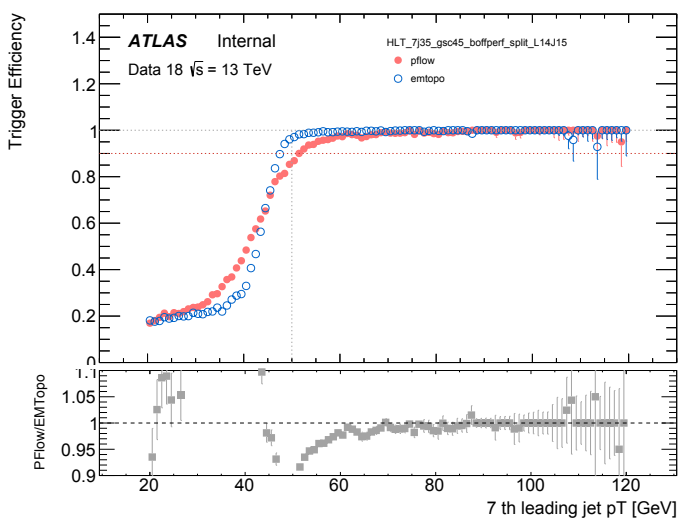

(f) 2018: 7j35_gsc45

Figura 4.11: Eficiencias, en datos, de los triggers de 5j, 6j, 7j y no pre-escaleados, utilizados para colectar los datos para el análisis, en función del momento transversal del último jet sobre el que se aplica la selección, para los jets EMTopo y PFlow. 


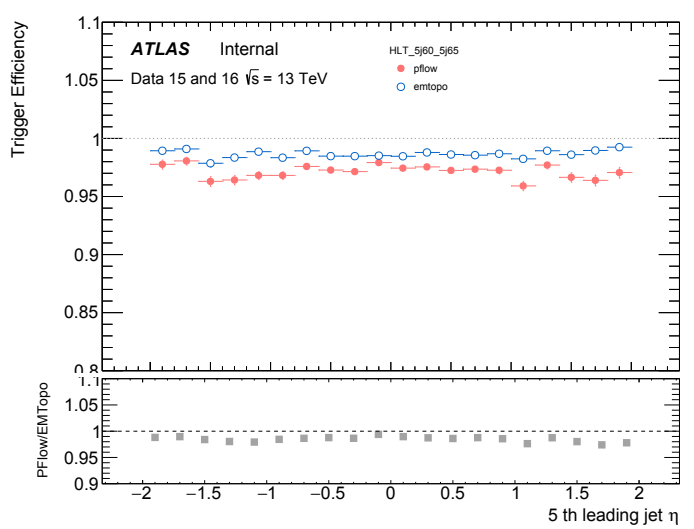

(a) 2015-2016: 5j60//5j65

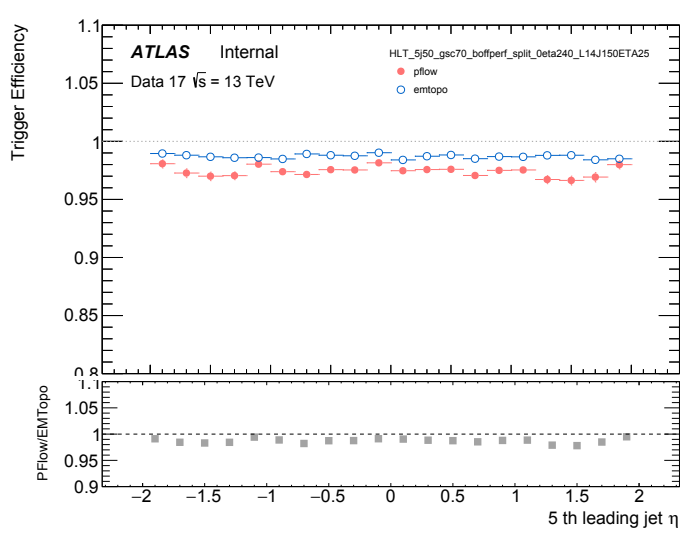

(c) 2017: 5j50_gsc70

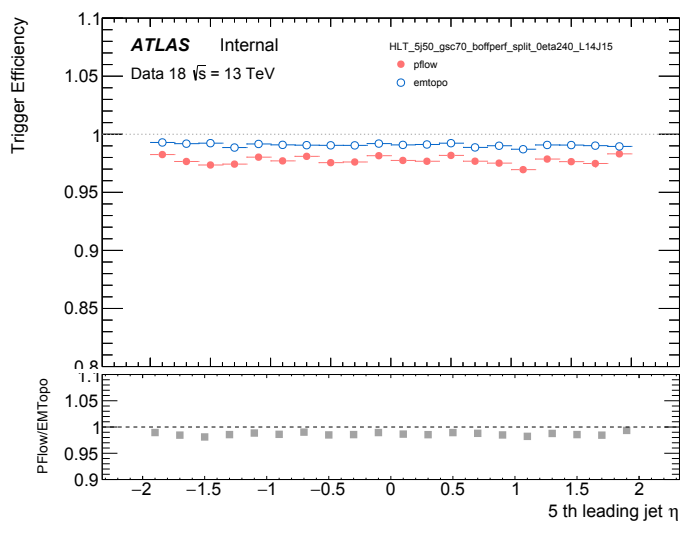

(e) 2018: 5j50_gsc70

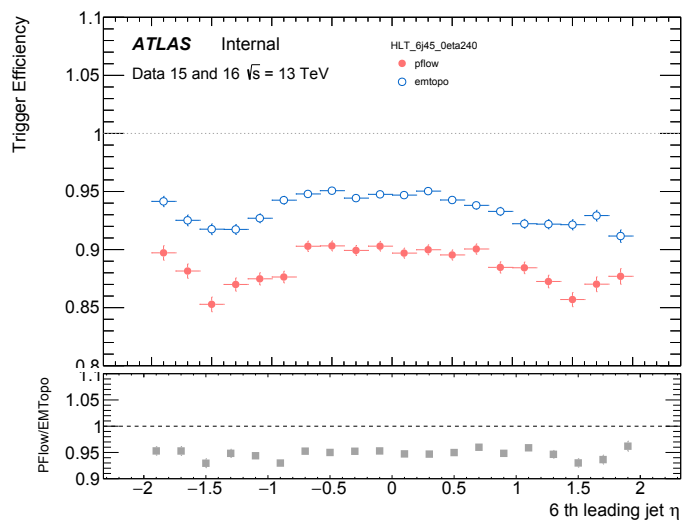

(b) $2015-2016: 6 \mathrm{j} 45$

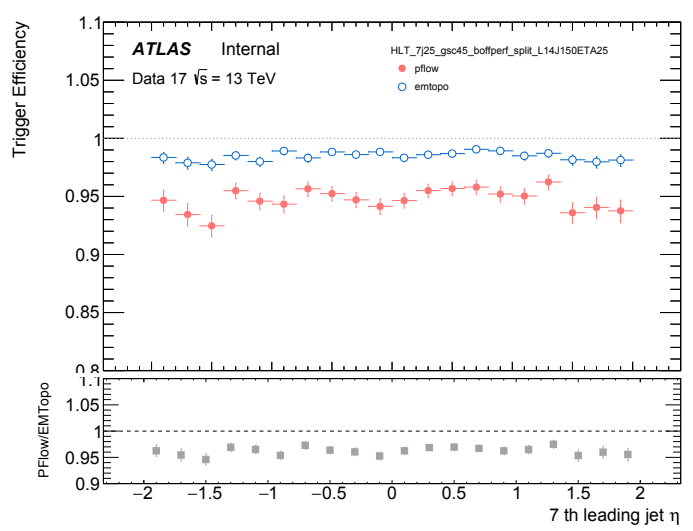

(d) 2017: 7j25_gsc45

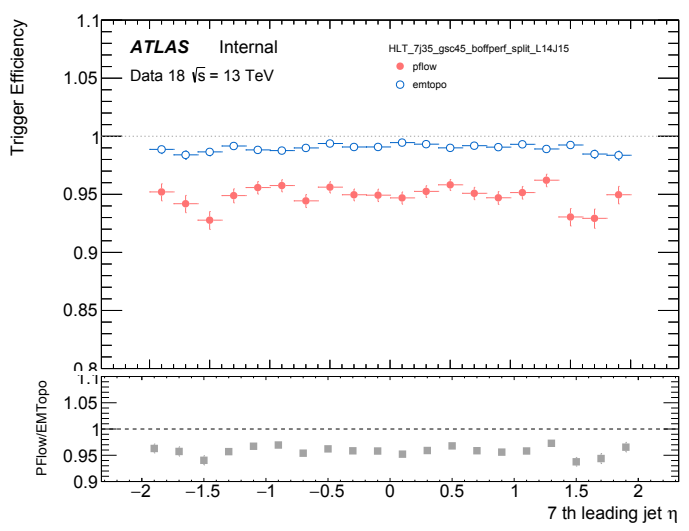

(f) 2018:7j35_gsc45

Figura 4.12: Eficiencias, en datos, de los triggers de $5 \mathrm{j}, 6 \mathrm{j}, 7 \mathrm{j}$ y no pre-escaleados, utilizados para colectar los datos para el análisis, en función de $\eta$ del último jet sobre el que se aplica la selección, para los jets EMTopo y PFlow. 

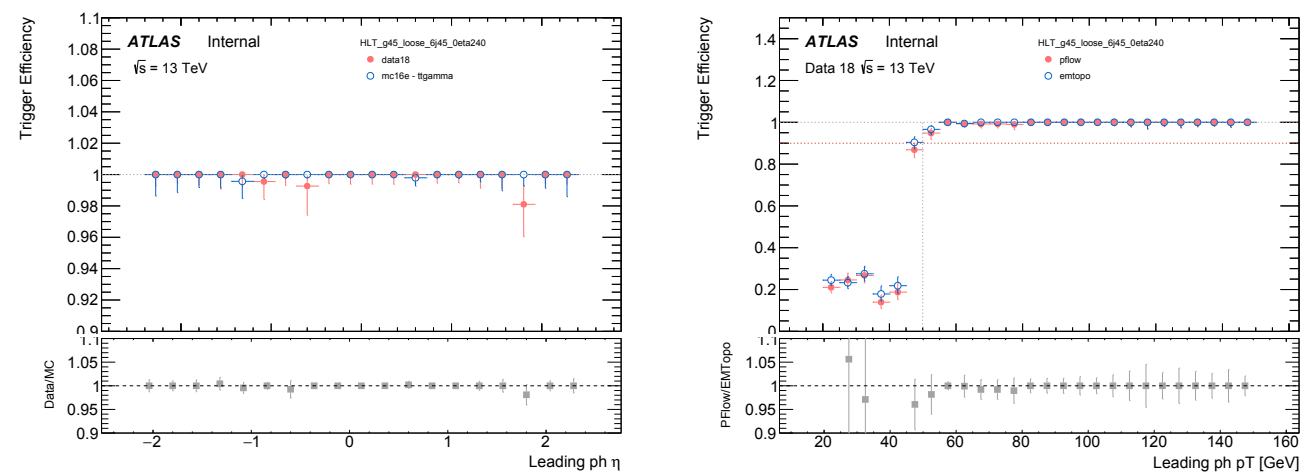

Figura 4.13: Eficiencias para el trigger HLT_g45_loose_6j45_0eta240 en datos 2018, para jets EMTopo y PFlow, en función de $\eta$ (izquierda) y $p_{\mathrm{T}}$ (derecha) del fotón leading. 


\section{Capítulo 5}

\section{Estrategia de búsqueda de SUSY en estados finales con fotones + jets + $E_{\mathrm{T}}^{\text {miss }}$}

Toda búsqueda de física más allá del SM representa básicamente un problema de clasificación entre señal y fondo. Se denomina señal a aquellos eventos provenientes de procesos propuestos por los nuevos modelos y teorías que se analizan, y los fondos son todos aquellos eventos producidos por procesos conocidos del SM con estados finales equivalentes a los buscados de nueva física. Para realizar esta clasificación, en el presente trabajo se buscan variables discriminatorias que nos permitan, en base a una selección sobre ellas, construir regiones en el espacio de parámetros dominadas por señal, naturalmente llamadas regiones de señal (SR). En base a esto, un exceso significativo de eventos respecto al fondo esperado implicaría la evidencia de nueva física. Dichos fondos son conocidos con una precisión limitada incluyendo todas las incertezas correspondientes, por lo que se vuelve crítico poder estimar correctamente su contribución en las SR. Para algunos de los fondos más relevantes para el análisis específico, predichos en base a simulaciones MC, se construyen regiones de control (CR) con datos en los que domina el fondo a determinar, de las que se extraen factores de transferencia utilizados para extrapolar los fondos desde las CR a las $\mathrm{SR}$, previo acuerdo en regiones de validación (VR). En eventos con estados finales dominados por objetos erróneamente reconstruidos debido a efectos instrumentales, las simulaciones dejan de ser confiables para su estimación. Esto se debe a que los eventos simulados no cuentan con el detalle necesario para predecir eventos extremos de las distintas distribuciones a alto $p_{\mathrm{T}}$ o $E_{\mathrm{T}}^{\text {miss }}$ particularmente. En estos casos, se prefieren técnicas basadas puramente en datos llamadas data-driven. Finalmente, para los fondos subdominantes, se utilizan las predicciones de los modelos incluidos en las simulaciones MC.

En este capítulo se describe la estrategia utilizada para la presente búsqueda, definiendo la muestra de datos a utilizar, la señal y los distintos fondos del SM para el estado final elegido que, en este análisis, se compone de al menos un fotón, jets y $E_{\mathrm{T}}^{\text {miss }}$. Luego se explicita la definición de los objetos físicos con los que se trabaja. En base a esto se definen las regiones de señal, control y validación, mostrando la optimización realizada para los datos de todo el Run-2, que se presenta en esta tesis por primera vez. Se sigue con una descripción de las técnicas data-driven utilizadas para estimar los fondos con fotones falsos (provenientes de una mala reconstrucción 
de electrones o jets), y finalmente se describe el tratamiento estadístico utilizado para la obtención de los resultados finales y su interpretación.

\subsection{Muestra de datos y selección de eventos}

El presente análisis se basa en la muestra de datos de colisiones protón-protón a $\sqrt{s}=13 \mathrm{TeV}$ de energía de centro de masa, registrados con el detector ATLAS entre 2015 y 2018, a una luminosidad integrada de $139 \mathrm{fb}^{-1}$.

La muestra de datos se recolectó usando el trigger de fotones HLT_g140_loose descripto en la Sección 4.2, que selecciona eventos con al menos un fotón que pase el criterio de identificación loose y con momento transversal mayor a $140 \mathrm{GeV}$. Dicho trigger fue el de menor requerimiento en $p_{\mathrm{T}} \mathrm{y}$ sin pre-escaleo durante toda la toma de datos del Run-2 ${ }^{1}$. Este trigger es completamente eficiente para fotones con $E_{\mathrm{T}}$ $>145 \mathrm{GeV}$ que satisfacen los cortes de selección de señal descriptos en el apartado 5.1.1. Se encontró que la eficiencia de dicho trigger fue $99.25 \pm 0.32 \%$, con relación a candidatos a fotones aislados con $E_{\mathrm{T}}>145 \mathrm{GeV}$. Esta eficiencia se calcula con el método Bootstrap descripto en la Sección 4.1. A partir de los resultados presentados en 4.2.1, se resume en la Figura 5.1 la eficiencia de este trigger en función de $E_{\mathrm{T}}, \eta$ y $\langle\mu\rangle$, respectivamente, para los distintos años de la toma de datos a fin de facilitar la comparación año a año. La incerteza total contempla la incerteza estadística debida al tamaño finito de la muestra Bootstrap y la incerteza sistemática del método. Se demuestra que el rendimiento del trigger se mantuvo estable a través de todo el Run-2, siendo robusto frente a pile-up y sin dependencias con $\eta$ (descartando la región del crack que no se utiliza para el análisis).

Los datos seleccionados por el trigger y los objetos correspondientes en dichos eventos, son luego preseleccionados en distintas etapas y almacenados en la derivación SUSY1 según se detalló anteriormente en la Sección 2.4.

En primer lugar, se seleccionan candidatos a fotón, leptón y jet con una serie de requisitos generales, llamados baseline. Luego de esta selección inicial, se aplica una eliminación de solapamiento para tratar el caso de una misma partícula que está siendo reconstruida como objetos diferentes por el detector. El momento transversal faltante se calcula a partir de los mencionados objetos baseline. Por último, los candidatos a fotones, leptones y jets utilizados para definir las diferentes regiones de control, validación y señal deben cumplir requisitos adicionales y se denominan, en lo sucesivo, "candidatos de señal".

En lo que sigue se describen los criterios de identificación elegidos para cada objeto que se utiliza en este análisis, en base a la descripción que se realizó en el Capítulo 3.

\subsubsection{Fotones}

En la selección offline, los candidatos a fotones deben pasar los criterios de identificación tight basados en la forma de la lluvia lateral y longitudinal que dejan en el calorímetro, pasar un umbral de $p_{\mathrm{T}}$ mayor a $25 \mathrm{GeV}$ y estar contenidos en un rango angular de $|\eta|<2.37$, descartando la región de transición barrel-endcap

\footnotetext{
${ }^{1}$ Vale aclarar que sólo para el año 2015, el umbral más bajo del trigger sin pre-escaleo fue el de HLT_g120_loose, pero HLT_g140_loose también se encontraba en el menú, por eso se eligió el segundo.
} 

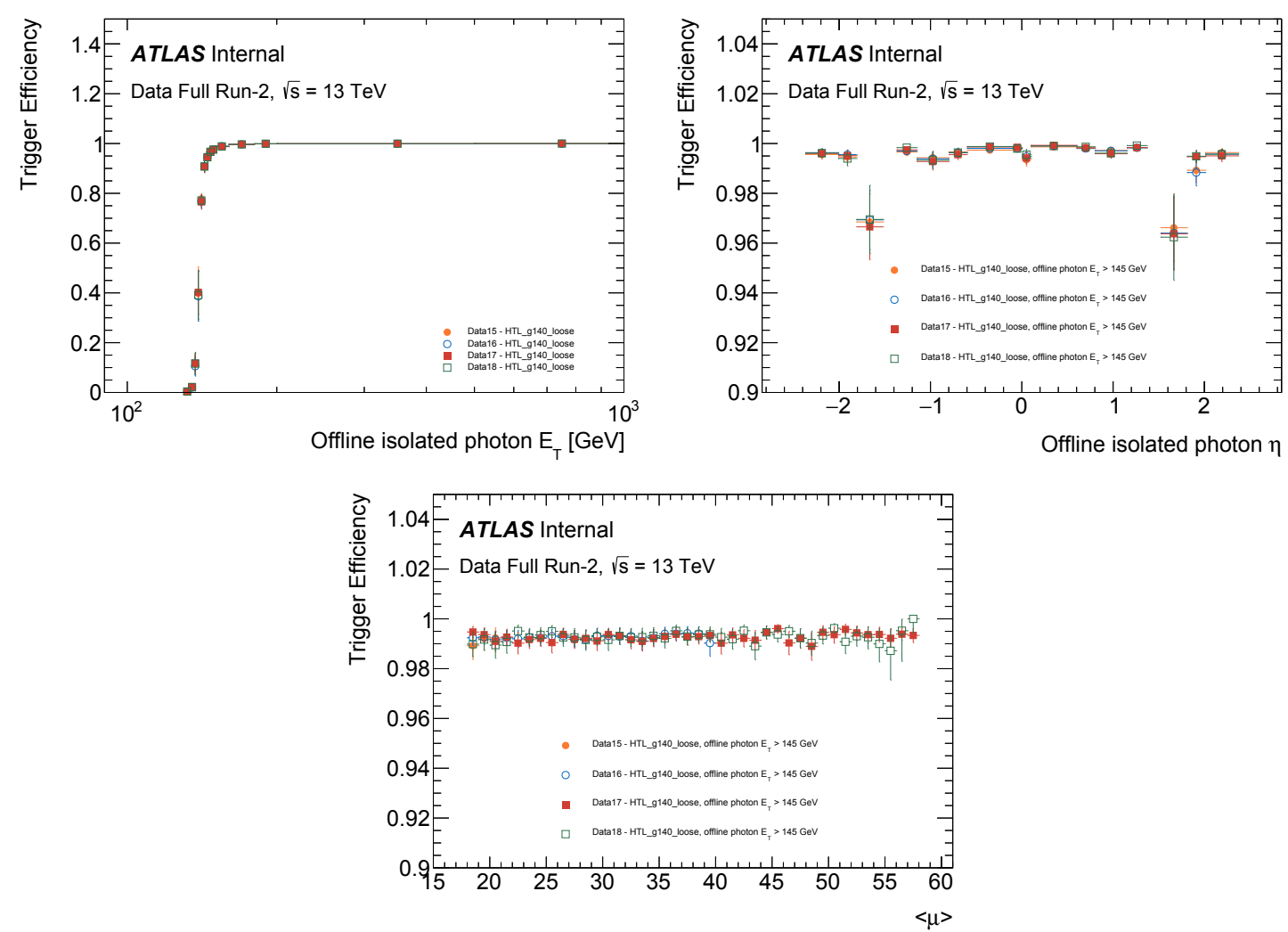

Figura 5.1: Eficiencias del trigger HLT_g140_loose en función de $E_{\mathrm{T}}, \eta \mathrm{y}\langle\mu\rangle$ para todos los datos de 2015 a 2018.

$(1.37<|\eta|<1.52)$. Un requisito adicional de $p_{\mathrm{T}}>75 \mathrm{GeV}$ se requiere para los fotones candidatos de señal y, en la selección final, el fotón principal siempre debe tener $p_{\mathrm{T}}>145 \mathrm{GeV}$, de manera de asegurar que fue seleccionado por el trigger. Se pide además que el fotón esté aislado, satisfaciendo los requerimientos del WP FixedCutTight (definido en el apartado 3.1.3), que aplica cortes tanto sobre la energía de aislamiento calorimétrica como sobre el aislamiento de trazas, lo que permite reducir el fondo proveniente de jets mal identificados como fotones.

\subsubsection{Electrones}

Los candidatos a electrones deben tener un valor de $p_{\mathrm{T}}>10 \mathrm{GeV},|\eta|<2.47$ y originarse en el vértice primario. Se impone un criterio de identificación loose, que se basa en las características de la lluvia EM, la calidad de la traza reconstruida asociada, y la cercanía entre la traza y la deposición de energía en el calorímetro.

Para los electrones candidatos de señal, se requiere que pasen el criterio de identificación tight y un criterio de aislamiento basado en el calorímetro y la traza (WP: FCLoose). Adicionalmente, se requiere que los electrones candidatos de señal tengan $p_{\mathrm{T}}>25 \mathrm{GeV}$ y $|\eta|<2.47$, excluyendo la región de transición barrel-endcap del ECAL. 


\subsubsection{Muones}

Los candidatos a muon se reconstruyen mediante una combinación de la información de la traza en el espectrómetro de muones y en el detector interno. Se requiere que los candidatos tengan un $p_{\mathrm{T}}>10 \mathrm{GeV}$ y $|\eta|<2.7$, que pasen el criterio de calidad medium y que se originen en el vértice primario. Los muones candidatos de señal se seleccionan entonces si $p_{\mathrm{T}}>25 \mathrm{GeV}$ y satisfacen un requisito de aislamiento similar al de los electrones (WP: FCLoose).

\subsubsection{Jets}

Los EMTopo jets se reconstruyen con el algoritmo anti- $k_{t}$ con $R=0.4$ utilizando clusters topológicos de celdas calorimétricas. La contribución media de energía esperada del pile-up se resta utilizando un factor que depende del área del jet. Los requisitos de selección basados en la traza se aplican para rechazar jets con un valor de $p_{\mathrm{T}}<60 \mathrm{GeV}$ y $|\eta|<2.4$, que se originan a partir de interacciones del pile-up [115]. A excepción del cálculo de $E_{\mathrm{T}}^{\text {miss }}$, donde no se aplica un requerimiento en $\eta$, los jets se conservan sólo si están en la región central del detector $(|\eta|<2.8)$ y con $p_{\mathrm{T}}>20 \mathrm{GeV}$ [116]. Los jets candidatos de señal son seleccionados con $p_{\mathrm{T}}>30 \mathrm{GeV}$ $\mathrm{y}|\eta|<2.5$.

Aunque los $b$-jets no se utilizan explícitamente para la selección del estado final de este análisis, son útiles en la definición de las regiones de control de las que se extrae la normalización MC para los procesos $W \gamma$ y $t \bar{t} \gamma$, como se describe en la Sección 5.3.2. Estos $b$-jets se identifican usando un algoritmo basado en la larga vida media, la alta multiplicidad de decaimiento y la gran masa de los $b$-hadrones en comparación con los quarks livianos y los gluones. El WP del algoritmo de etiquetado de $b$-jets utilizado en este análisis tiene una eficiencia nominal del $77 \%$.

\subsubsection{Eliminación de solapamiento}

Los algoritmos de reconstrucción e identificación empleados para los distintos tipos de partículas pueden, simultáneamente, identificar como propio a un mismo objeto, siendo correcta sólo una de dichas identificaciones. En base a esto, un objeto podría ser contado múltiples veces en el evento, situación que se debe evitar. Por lo tanto, se aplica un procedimiento para eliminar estas superposiciones [117, 118, $119,120]$ entre objetos preseleccionados, antes de que se impongan los requisitos de aislamiento correspondientes.

La estrategia básica y el orden de eliminación es el siguiente (ver [121, 122] para una descripción más detallada):

- los muones CT (calo-etiquetados) que comparten una traza con un electrón preseleccionado se eliminan.

- los electrones preseleccionados que comparten una traza con los restantes muones se eliminan.

- si un fotón y un electrón se encuentran dentro de $\Delta R<0.4$, el objeto es interpretado como un electrón, y el fotón es eliminado.

- los fotones preseleccionados son eliminados si su distancia al muón más cercano es $\Delta R<0.4$. 
- los jets que están angularmente cerca $(\Delta R<0.2)$ de un electrón preseleccionado, son eliminados.

- los electrones preseleccionados son eliminados si su distancia al jet más cercano es $\Delta R<0.4$.

- los jets con un número de trazas menor a 3 y angularmente cerca $(\Delta R<0.2)$ a un muón preseleccionado, son eliminados.

- los muones preseleccionados son eliminados si su distancia al jet más cercano es $\Delta R<0.4$.

- los jets que están angularmente cerca $(\Delta R<0.4)$ a un fotón preseleccionado, son eliminados.

\subsubsection{Momento transverso faltante}

El momento transverso faltante se calcula con un algoritmo basado en objetos. Los depósitos de energía en el calorímetro se asocian a objetos de alto $p_{\mathrm{T}}$ en el siguiente orden: electrones, fotones, jets y muones. Las trazas no asociadas a ninguno de estos objetos están incluidas en el llamado Soft Term. Las componentes x e y de $E_{\mathrm{T}}^{\text {miss }}$ se obtienen de la suma de los siguientes términos, como se describió en la Sección 3.4:

$$
E_{x(y)}^{\text {miss }}=E_{x(y)}^{\text {misse }}+E_{x(y)}^{\text {miss } \gamma}+E_{x(y)}^{\text {missjet }}+E_{x(y)}^{\text {miss } \mu}+E_{x(y)}^{\text {miss Soft Term }},
$$

donde cada término se calcula a partir de la suma negativa de los objetos reconstruidos calibrados en la categoría respectiva.

\subsection{Muestras MC de señal y de fondos del SM}

En este análisis se simulan muestras de las señales SUSY y de los fondos del modelo estándar esperados, usando generadores dedicados de MC a $\sqrt{s}=13 \mathrm{TeV}$. Todas las muestras se pasan a través de una simulación completa del detector ATLAS como se describe en la Sección 2.6, y se reconstruyen con los mismos algoritmos utilizados para los datos. Las muestras se generan con la distribución prevista de pile-up para cada conjunto de datos de 2015-2016, 2017 y 2018, identificadas como MC16a, MC16d y MC16e, respectivamente, a las que se le aplica un repesado evento a evento para modelar realísticamente las condiciones de la toma de datos, haciendo coincidir la distribución simulada del número de colisiones inelásticas $p p$ por cruce de paquetes de protones con la observada en datos.

\subsubsection{Muestras de señal}

Debido a la gran cantidad de parámetros libres que tiene el MSSM ( 100), y la imposibilidad manifiesta de realizar actualmente una búsqueda orientada por un modelo tan general, la estrategia de las grandes colaboraciones del LHC para las búsquedas de SUSY es la de utilizar modelos simplificados con un número reducido de parámetros libres que puedan entonces ser acotados por las medidas experimentales. 
Como se detalla en el Capítulo 1, la búsqueda de SUSY que se realiza en la presente tesis está motivada por modelos GGM que predicen un estado final que consta de al menos un fotón, jets y un alto valor de $E_{\mathrm{T}}^{\text {miss }}$. En particular, dichas características provienen del decaimiento del neutralino más liviano $\left(\widetilde{\chi}_{1}^{0}\right)$ que es la NLSP de la teoría, siendo el gravitino $(\widetilde{G})$ la LSP y fuente de $E_{\mathrm{T}}^{\mathrm{miss}}$. El $\widetilde{\chi}_{1}^{0}$ es una mezcla bino-higgsino con parámetro $\mu>0$ (parámetro de masa de los higgsinos), cuya componente bino se acopla tanto al fotón como al bosón $Z$.

Se considera al gluino $(\tilde{g})$, cuya producción de a pares inicia la cadena de decaimiento, como la única spartícula con color relevante, para establecer límites en su masa. Las masas de todos los squarks se imponen en $5 \mathrm{TeV}$, desacoplándolos o haciéndolos inaccesibles en su estado de masa on-shell, resultando entonces en que cada gluino decaiga a través de un squark virtual (los doce autoestados de sabo$\mathrm{r}$ /quiralidad del squark se consideran completamente degenerados) a pares quarkantiquark más el neutralino NLSP (Figura 5.2 derecha), o a pares quark-antiquark más un chargino que, a su vez, decae al neutralino NLSP (Figura 5.2 izquierda), dependiendo de la diferencia de masas entre el $\tilde{g}$ y el $\widetilde{\chi}_{1}^{0}$. Estas características del decaimiento del gluino, sus BRs a neutralinos o charginos y las diferencias de masas, son particularmente importantes para optimizar su selección y definir las distintas regiones de señal, ya que se tendrán diferentes multiplicidades de jets y diferentes momentos transversos en el estado final, permitiendo una serie de diferentes cortes discriminantes.

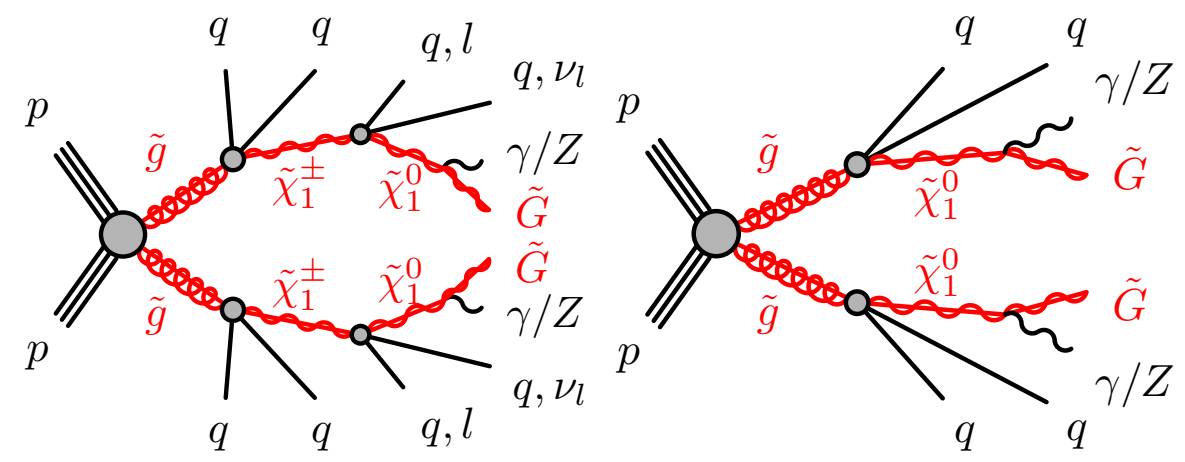

Figura 5.2: Diagramas de producción de gluinos y posterior decaimiento a un estado final con al menos un fotón, jets y momento transversal faltante. La cadena de la izquierda, con mayor cantidad de jets, se obtiene para diferencia de masas grandes entre el gluino y el neutralino NLSP. La cadena corta (derecha) representa un escenario de masas comprimido.

Otros parámetros del modelo se determinan para favorecer el decaimiento antes mencionado, siendo éstos los definidos en la Sección 1.4.2, y se consideran como $M_{2}=3 \mathrm{TeV}, \tan \beta=1.5$ y $m_{\tilde{G}}=1 \mathrm{eV}$. Todos los términos de acoplamiento trilineal se ajustan a cero y las masas de los sleptones se desacoplan con una masa de $5 \mathrm{TeV}$.

De los bosones de Higgs, tanto los escalares neutro pesado $\left(H^{0}\right)$ y los cargados $\left(H^{ \pm}\right)$, como el pseudoescalar $\left(A^{0}\right)$ están en el régimen de desacoplamiento con sus masas en $2 \mathrm{TeV}$, mientras que la masa del escalar neutro $h^{0}$ se establece en $125 \mathrm{GeV}$. Este último es el bosón que se considera usualmente más liviano en la teoría, cuya masa se asocia con el valor medido para el bosón de Higgs del SM en el LHC [123]. En los escenarios de SUSY con mediadores de gauge, existen varios mecanismos 
[124, 125, 126, 127, 128] para generar una masa del bosón de Higgs con este valor observado, sin cambiar la fenomenología de los modelos aquí considerados. Además, se ajustó $c \tau_{\mathrm{NLSP}}<0.1 \mathrm{~mm}$ para asegurar que el neutralino decaiga antes de alcanzar el detector, y se prohíbe el decaimiento directo del gluino, ajustando $B R(\tilde{g} \rightarrow \widetilde{G} g)=$ 0 .

Los parámetros $M_{1}$ y $\mu$, que determinan la masa del neutralino más liviano, se establecen de tal manera que los BR del $\widetilde{\chi}_{1}^{0}$ sean aproximadamente constantes, y provean los estados finales de interés. En particular, el valor de $\mu$ se toma mayor a cero para desfavorecer el decaimiento del $\widetilde{\chi}_{1}^{0}$ al bosón de Higgs, y de tal manera que se maximice el estado final de un fotón y jets livianos. Por lo tanto se tiene $\operatorname{BR}\left(\widetilde{\chi}_{1}^{0} \rightarrow \gamma \widetilde{G}\right) \sim 50 \%, \operatorname{BR}\left(\widetilde{\chi}_{1}^{0} \rightarrow Z \widetilde{G}\right) \sim 49 \%$ y $\operatorname{BR}\left(\widetilde{\chi}_{1}^{0} \rightarrow h \widetilde{G}\right) \sim 1 \%$, números que pueden variar en $\pm 1 \%$ para cualquier valor de $\mu$, como puede verse en la Figura 5.3.

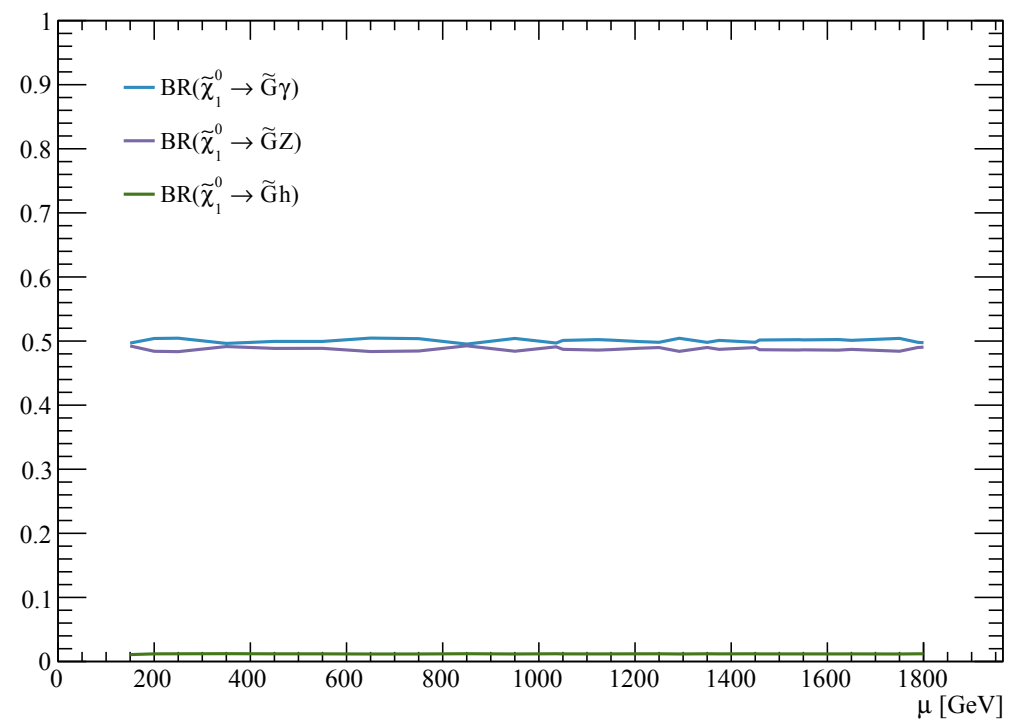

Figura 5.3: Probabilidad de decaimiento del $\widetilde{\chi}_{1}^{0}$ a un fotón, a un bosón $Z$ y a un bosón de Higgs, en función del parámetro de masa de los higgsinos $(\mu)$.

Con esta configuración, el $\sim 25 \%$ de los eventos tendrán dos fotones en el estado final, por lo que se hace una búsqueda inclusiva que requiere al menos un $\gamma$, más dos $\widetilde{G}$ en el estado final. La búsqueda con explícitamente dos fotones en estado final, donde el neutralino es bino puro $(\mu=0)$ y $\operatorname{BR}\left(\widetilde{\chi}_{1}^{0} \rightarrow \gamma \widetilde{G}\right)=100 \%$ es cubierta por otro estudio según se detalla en [47].

Por lo tanto, $M_{3}$ (relacionada con la masa del gluino) y $\mu$ (relacionada con la masa del neutralino) son los únicos dos parámetros libres del modelo, y para esta búsqueda se considera el rango de valores tales que, $150 \mathrm{GeV}<m_{\widetilde{\chi}_{1}^{0}} \leq\left(m_{\tilde{g}}-50\right) \mathrm{GeV}$ y $1400 \mathrm{GeV}<m_{\tilde{g}}<2600 \mathrm{GeV}$.

Para igualar las masas de los gluinos a los correspondientes archivos $\mathrm{LHE}^{2}$ dis-

\footnotetext{
${ }^{2}$ Los archivos LHE contienen la generación de eventos, realizada en MadGraph para la producción de pares de gluinos (en este caso), que luego se utiliza en Pythia para finalizar la simulación del modelo particular. En ATLAS se sugiere usar dichos archivos para ahorrar tiempo de cómputo, compartiendo esta generación entre los distintos análisis que usen gluinos, y homogeneizando los valores de las masas.
} 


\begin{tabular}{rrr}
\hline$m_{\tilde{g}}[\mathrm{GeV}]$ & $\sigma\left(\mathrm{NNLO}_{\text {approx }}+\mathrm{NNLL}\right)[\mathrm{pb}]$ & Incerteza $(\%)$ \\
\hline 1400 & $2.84 \times 10^{-2}$ & 14.44 \\
1600 & $8.87 \times 10^{-3}$ & 15.94 \\
1800 & $2.93 \times 10^{-3}$ & 17.56 \\
2000 & $1.01 \times 10^{-3}$ & 19.42 \\
2200 & $3.56 \times 10^{-4}$ & 21.83 \\
2400 & $1.28 \times 10^{-4}$ & 25.19 \\
2600 & $4.62 \times 10^{-5}$ & 30.31 \\
\hline
\end{tabular}

Tabla 5.1: La sección eficaz total a $\mathrm{NNLO}_{\text {approx }}+$ NNLL, con sus incertezas, para los puntos de señal como función de $m_{\tilde{g}}$.

ponibles, no se tienen en cuenta correcciones radiativas a las masas, lo que implica que las masas de los gluinos ahora coinciden con los valores de los parámetros $M_{3}$.

Los puntos de señal generados se resumen en la Figura 5.4. Consiste en 81 puntos de producción fuerte $(\tilde{g} \tilde{g})$ generados con 10k eventos cada uno. Además, 26 muestras fueron simuladas a nivel generador (MC TRUTH sin incluir el detector) para estudios futuros a mayor luminosidad o sensibilidad.

Las secciones eficaces se calculan a NNLO $_{\text {approx }}+$ NNLL [129, 130, 131, 132, 133]. La sección eficaz nominal y las incertezas se toman de un envelope de predicciones de secciones eficaces utilizando diferentes conjuntos de PDF y escalas de factorización y renormalización, como se describe en [134]. La sección eficaz para los diferentes puntos de señal se resumen en la Tabla 5.1.

A partir de las suposiciones y simplificaciones anteriores, el conjunto de parámetros obtenido se utiliza para calcular el espectro completo de masas, las probabilidades y los anchos de decaimiento de gluinos y neutralinos utilizando SUSPECT v2.43 [135], SDECAY v1.5 [136] y HDECAY v3.4 [137], que se ejecutan como parte del conjunto de programas SUSYHIT v1.5a [138]. En particular, SUSPECT permite calcular el espectro de partículas supersimétricas en el MSSM, y puede hacerlo para distintos modelos como mSUGRA, AMSB o los particularmente interesantes para la presente tesis, con rompimiento de supersimetría mediada por campos de gauge, GMSB. SDECAY calcula las probabilidades y los anchos de decaimiento para todas las partículas supersimétricas en el MSSM, incluyendo efectos de orden superior, mientras que HDECAY los calcula para el bosón de Higgs del SM y de las partículas neutras y cargadas del sector de Higgs en el MSSM. Finalmente, SUSYHIT es la interfase que permite trabajar directa y eficientemente con las rutinas anteriores.

Todas las muestras de la señal fueron generadas utilizando los archivos LHE (generados centralmente) para la producción de gluinos, con MADGRAPH5 interconectado a PYTHIA8, y con la simulación rápida del detector ATLAS provista por ATLFAST-II [139].

\subsubsection{Muestras de fondo}

Varios procesos del SM pueden imitar una señal SUSY con fotones, jets y momento transversal faltante. Dichos procesos surgen de eventos con fotones reales, o de eventos en los que un electrón o un jet se identifica erróneamente como un fotón. Se espera que la primera fuente de fondo reciba contribuciones de eventos en los que se produzca un bosón $W$ o $Z$ o un par $t \bar{t}$ en asociación con al menos un fotón 


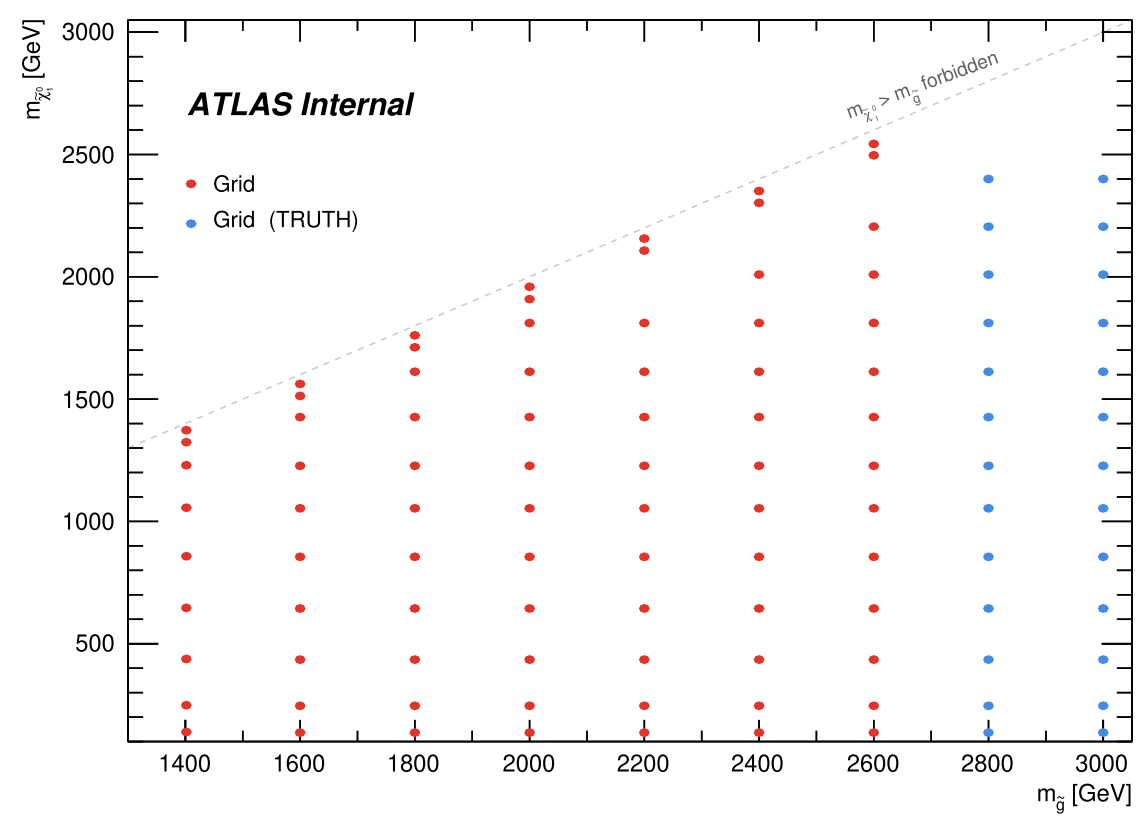

Figura 5.4: Muestras de la señal GGM para la producción fuerte $\tilde{g} \tilde{g}$. Cada punto de señal está definido por las masas del gluino y del neutralino. Los puntos celestes, para masas del gluino de 2800 y $3000 \mathrm{GeV}$, fueron producidos solo a nivel generador para estudios futuros.

real, con neutrinos en los subsiguientes decaimientos débiles, que proporcionen $E_{\mathrm{T}}^{\text {miss }}$ significativo. Estas fuentes de fondo se indican como $W \gamma, W \gamma \gamma, Z \gamma, Z \gamma \gamma$ y $t \bar{t} \gamma$. Los eventos con fotones reales también pueden contribuir al fondo cuando una cantidad significativa de $E_{\mathrm{T}}^{\text {miss }}$ surge de fuentes instrumentales.

Los fondos $W \gamma, t \bar{t} \gamma$ y $\gamma+$ jets se estiman escalando la correspondiente muestra de $\mathrm{MC}$ para que coincida con el número de eventos observados en las correspondientes regiones de control (CR) enriquecidas en el proceso de fondo dado, pero que, por lo demás, son cinemáticamente similares a las correspondientes regiones de señal (SR).

Las contribuciones al fondo menos relevantes como $\gamma \gamma, W \gamma \gamma, Z \gamma$ y $Z \gamma \gamma$ se estiman directamente a partir de simulaciones MC. La contaminación de fondos de fotones falsos debido a la identificación errónea de electrones y jets, provenientes de eventos $W+$ jets, $Z+$ jets, $t \bar{t}$ o multijets se estima de una manera data-driven, como se discute en la Sección 5.4. La Tabla 5.2 resume las principales fuentes de fondo consideradas.

\begin{tabular}{|c|c|c|}
\hline & $E_{\mathrm{T}}^{\text {miss }}$ Real & $E_{\mathrm{T}}^{\text {miss }}$ Instrumental \\
\hline \multirow{2}{*}{ Fotón Real } & $Z(\nu \nu) \gamma, W \gamma$ & $\gamma+$ jets, $\gamma \gamma, Z(l l) \gamma, Z(l l) \gamma \gamma$ \\
& $t \bar{t} \gamma, Z(\nu \nu) \gamma \gamma, W \gamma \gamma$ & Fotón Fake \\
\hline F jets, $Z(\nu \nu)+$ jets, $t \bar{t}$ & multijet, $Z(l l)+$ jets \\
\hline
\end{tabular}

Tabla 5.2: Procesos del Modelo Estándar que contribuyen al fondo.

La producción de $W \gamma, W \gamma \gamma, Z \gamma, Z \gamma \gamma, \gamma \gamma$ y $\gamma+$ jets se simula usando SHERPA 2.2 [140]. Los elementos de la matriz se calculan para un máximo de cuatro partones a LO y se fusionan con la lluvia de partones de SHERPA [141] utilizando la prescripción de MEPS@LO [142]. El set de PDF CT10 [143] se usa en conjunto con un 
parton shower tuning dedicado, desarrollado por los autores de SHERPA.

La muestra de eventos $t \bar{t} \gamma$ se genera con MG5_aMC@NLO [144] a segundo orden en teoría de perturbaciones (NLO), con Pythia 8 para el modelo de la lluvia de partones [145].

Las muestras simuladas para los fondos del SM considerados en esta búsqueda se resumen en las Tablas 5.3, 5.4, 5.5 y 5.6.

Tabla 5.3: Detalle de las muestras MC consideradas como fondos del SM $(t \bar{t} \gamma)$

\begin{tabular}{llr}
\hline$t \bar{t} \gamma$ & Generador & Sección eficaz [pb] \\
\hline$t \bar{t} \gamma, p_{\mathrm{T}}^{\gamma}>140 \mathrm{GeV}$ & MADGRAPH/PYTHIA8 & 0.21551 \\
\hline
\end{tabular}

Tabla 5.4: Detalle de las muestras MC consideradas como fondos del SM $(W / Z \gamma)$

\begin{tabular}{llr}
\hline$V \gamma$ & Generador & Sección eficaz [pb] \\
\hline$Z(e e) \gamma, p_{\mathrm{T}}^{\gamma}>140 \mathrm{GeV}$ & SHERPA v2.2.2 & 0.063397 \\
\hline$Z(\mu \mu) \gamma, p_{\mathrm{T}}^{\gamma}>140 \mathrm{GeV}$ & SHERPA v2.2.2 & 0.063172 \\
\hline$Z(\tau \tau) \gamma, p_{\mathrm{T}}^{\gamma}>140 \mathrm{GeV}$ & SHERPA v2.2.2 & 0.063407 \\
\hline$Z(\nu \nu) \gamma, p_{\mathrm{T}}^{\gamma}>140 \mathrm{GeV}$ & SHERPA v2.2.2 & 0.24455 \\
\hline$W(e \nu) \gamma, p_{\mathrm{T}}^{\gamma}>140 \mathrm{GeV}$ & SHERPA v2.2.2 & 0.29803 \\
\hline$W(\mu \nu) \gamma, p_{\mathrm{T}}^{\gamma}>140 \mathrm{GeV}$ & SHERPA v2.2.2 & 0.2987 \\
\hline$W(\tau \nu) \gamma, p_{\mathrm{T}}^{\gamma}>140 \mathrm{GeV}$ & SHERPA v2.2.2 & 0.29827 \\
\hline
\end{tabular}

Tabla 5.5: Detalle de las muestras MC consideradas como fondos del SM $(\gamma+$ jets $)$

\begin{tabular}{llr}
\hline$\gamma+$ jets & Generador & Sección eficaz $[\mathrm{pb}]$ \\
\hline$p_{\mathrm{T}}^{\gamma} \in[35-70] \mathrm{GeV}$ & SherPA v2.2.2 & 43976.0 \\
$p_{\mathrm{T}}^{\gamma} \in[70-140] \mathrm{GeV}$ & SHERPA v2.2.2 & 4526.5 \\
$p_{\mathrm{T}}^{\gamma} \in[140-280] \mathrm{GeV}$ & SHERPA v2.2.2 & 376.05 \\
$p_{\mathrm{T}}^{\gamma} \in[280-500] \mathrm{GeV}$ & SHERPA v2.2.2 & 21.851 \\
$p_{\mathrm{T}}^{\gamma} \in[500-1000] \mathrm{GeV}$ & SHERPA v2.2.2 & 1.4637 \\
$p_{\mathrm{T}}^{\gamma} \in[1000-] \mathrm{GeV}$ & SHERPA v2.2.2 & 0.029867 \\
\hline
\end{tabular}

Tabla 5.6: Detalle de las muestras MC consideradas como fondos del SM (di-fotón)

\begin{tabular}{llr}
\hline Difotón & Generador & Sección eficaz [pb] \\
\hline$Z(e e) \gamma \gamma, p_{\mathrm{T}}^{\gamma}>140 \mathrm{GeV}$ & SHERPA v2.2.2 & $9.911 \times 10^{-3}$ \\
$Z(\mu \mu) \gamma \gamma, p_{\mathrm{T}}^{\gamma}>140 \mathrm{GeV}$ & SHERPA v2.2.2 & $10.59 \times 10^{-3}$ \\
$Z(\tau \tau) \gamma \gamma, p_{\mathrm{T}}^{\gamma}>140 \mathrm{GeV}$ & SHERPA v2.2.2 & $28.36 \times 10^{-3}$ \\
$Z(\nu \nu) \gamma \gamma, p_{\mathrm{T}}^{\gamma}>140 \mathrm{GeV}$ & SHERPA v2.2.2 & $4.836 \times 10^{-3}$ \\
\hline$W(e \nu) \gamma \gamma, p_{\mathrm{T}}^{\gamma}>140 \mathrm{GeV}$ & SHERPA v2.2.2 & $15.54 \times 10^{-3}$ \\
$W(\mu \nu) \gamma \gamma, p_{\mathrm{T}}^{\gamma}>140 \mathrm{GeV}$ & SHERPA v2.2.2 & $15.23 \times 10^{-3}$ \\
$W(\tau \nu) \gamma \gamma, p_{\mathrm{T}}^{\gamma}>140 \mathrm{GeV}$ & SHERPA v2.2.2 & $14.75 \times 10^{-3}$ \\
\hline
\end{tabular}




\subsection{Regiones de señal, control y validación}

Como se mencionó al inicio de este capítulo, la presente búsqueda representa un problema de clasificación de datos entre eventos provenientes de SUSY (señal) y aquellos del SM que imitan el estado final (fondo). Un exceso de eventos de señal con respecto al fondo esperado en una dada región del espacio de parámetros podría significar la comprobación de la nueva teoría. Por lo tanto, para resolver este tipo de problemas se definen una serie de variables discriminatorias que nos permitan seleccionar eventos distinguiendo su origen, a partir de una selección sobre dichas variables. Un conjunto de cortes que seleccionan, mayoritariamente, solo eventos de señal forman una región de señal (SR). Sin embargo, debido a la naturaleza de los experimentos con los que se trabaja, se espera una contaminación de fondo en dichas SR y su correcta estimación es de vital importancia para evaluar cualquier desviación entre datos y las predicciones.

Para estimar los fondos del SM se cuenta con técnicas data-driven (y se describen en la Sección 5.4) o con muestras de MC como las descriptas en la sección anterior. Los fondos que se estiman a partir de estas muestras MC, se dividen en fondos secundarios y en fondos principales. Para los primeros, debido a la baja contribución, su estimación se hace directamente de la predicción de MC. Para los fondos principales, debido a su importancia y, al mismo tiempo, a la limitada precisión de los cálculos incorporados en los $\mathrm{MC}$ y, por ende, que pueden no reproducir con la precisión deseada lo observado en datos, se procede con una estrategia diferente. Se espera que los generadores de estas muestras MC puedan reproducir correctamente la forma de las distribuciones, pero no así su normalización. Para validar las muestras MC y extraer la normalización de lo fondos principales, se construyen regiones ortogonales a las SR, pero cinemáticamente cercanas y que presenten una cantidad de eventos de señal despreciable, llamadas regiones de control (CR). Vale aclarar aquí que dos regiones se consideran ortogonales y cercanas, si comparten la gran mayoría de los cortes de selección, pero hay al menos uno invertido, generando que no haya superposición entre los conjuntos de eventos seleccionados por una y otra. Las CR, cada una diseñada específicamente para un dado fondo (es decir, enriquecidas en ese dado fondo), permiten comparar el número de eventos observado en datos y en MC, y extraer factores de transferencia vía un ajuste simultáneo en cada región, usados luego para extrapolar cada fondo desde las CR a las SR.

Finalmente, se deben validar los resultados obtenidos. Para esto, se definen otras regiones que se encuentran entre las regiones de control y las de señal, llamadas regiones de validación (VR). Dichas regiones son ortogonales a las CR y SR, y se definen para estar lo suficientemente cerca (cinemáticamente) de las SR para que la validación de la extrapolación sea válida, pero al mismo tiempo, manteniendo la contaminación de señal lo más baja posible, de manera que la señal no altere sus distribuciones. Se vuelve sobre esta discusión en la Sección 5.5, donde se detalla explícitamente la forma de los factores mencionados, los ajustes y el modelo estadístico utilizado.

En las secciones siguientes se muestra un estudio detallado de la optimización y definición de las regiones de señal junto a una descripción de las regiones de control y validación. En la Figura 5.5, se presenta un ejemplo de una CR con su VR para una $\mathrm{SR}$, en el plano de las variables $E_{\mathrm{T}}^{\text {miss }}$ y $H_{\mathrm{T}}$ que, como se verá a continuación, son dos de las principales variables discriminadoras para esta búsqueda. Es importante 
remarcar que las distintas regiones están definidas en un espacio $\mathrm{N}$ dimensional, siendo $\mathrm{N}$ el número de variables discriminatorias utilizadas para definirlas, por lo que la presente figura es sólo un esquema que permite la visualización.

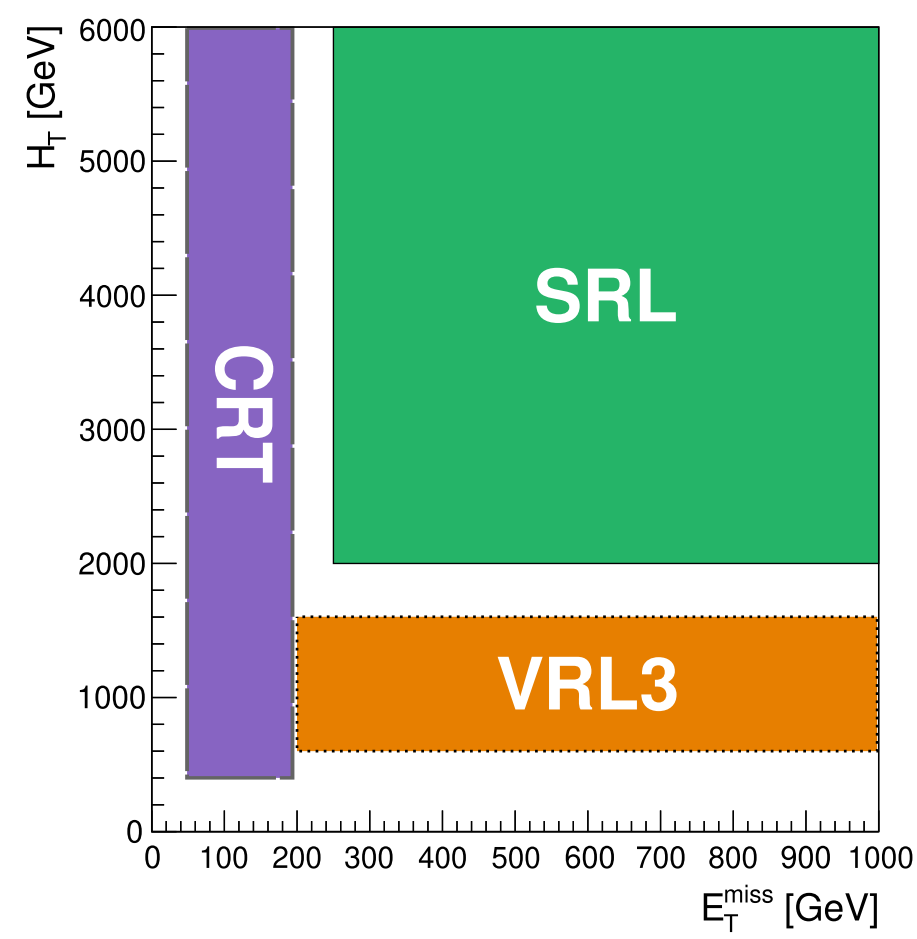

Figura 5.5: Ejemplo de una CR con una de sus VR para una dada SR (todas ortogonales entre sí), en el plano de las variables $E_{\mathrm{T}}^{\text {miss }}$ y $H_{\mathrm{T}}$. Dichas regiones están definidas en un espacio $\mathrm{N}$ dimensional (siendo $\mathrm{N}$ el número de variables discriminatorias utilizadas para definirlas), por lo que el presente esquema es solo demostrativo.

\subsubsection{Optimización y definición de las regiones de señal}

Las definiciones de las regiones de señal deben ser tales que estén enriquecidas de señal, minimizando la contaminación de fondo. Al mismo tiempo, se intenta que los cortes de selección no sean demasiado estrictos y específicos para un único modelo, para que la búsqueda se realice de la manera más independiente de modelos posible. Dichos cortes se eligen optimizando la significancia de descubrimiento esperada, que se define como:

$$
Z=\sqrt{2\left(n \ln \left[\frac{n\left(b+\sigma_{b}^{2}\right)}{b^{2}+n \sigma_{b}^{2}}\right]-\frac{b^{2}}{\sigma_{b}^{2}} \ln \left[1+\frac{\sigma_{b}^{2}(n-b)}{b\left(b+\sigma_{b}^{2}\right)}\right]\right)}
$$

donde $n$ es la suma de los eventos de señal $(s)$ y fondo $(b)$, y $\sigma_{b}$ es la incerteza en el fondo. Si se desarrollan los logaritmos de la expresión anterior, se la puede reescribir como

$$
Z=\frac{s}{\sqrt{b+\sigma_{b}^{2}}}\left(1+\mathcal{O}\left(\frac{s}{b}\right)+\mathcal{O}\left(\frac{\sigma_{b}^{2}}{b}\right)\right)
$$


que en el caso para $s<<b$ y $\sigma_{b}^{2}<<b$, se convierte en la cantidad utilizada usualmente: $s / \sqrt{b}$.

En la presente optimización, todas las muestras de MC mencionadas en el apartado 5.2.2, normalizadas a $139 \mathrm{fb}^{-1}$, se utilizan como fondo. Se requiere que en cada SR haya al menos tres eventos de señal y al menos uno de fondo, y se le asigna un $30 \%$ de incerteza a la estimación del fondo.

La optimización se inicia con la mínima selección necesaria para obtener el estado final buscado, con los datos y objetos disponibles durante la toma de datos del Run2 , requiriendo eventos con al menos un fotón aislado con $p_{\mathrm{T}}>145 \mathrm{GeV}$, más de un jet con $p_{\mathrm{T}}>30 \mathrm{GeV}$, veto de leptones y presencia de $E_{\mathrm{T}}^{\text {miss }}$. El requerimiento mínimo en el $p_{\mathrm{T}}$ del fotón depende exclusivamente del trigger utilizado. Para la selección del $p_{\mathrm{T}}$ de los jets, se utilizan los valores recomendados para su óptima reconstrucción, siendo lo más inclusivos posibles para no sesgar innecesariamente la búsqueda. En ATLAS se realiza también un análisis que busca estados finales con un fotón, un leptón y $E_{\mathrm{T}}^{\text {miss }}$ [45], por lo que se decide aplicar un veto de leptones en esta búsqueda para no solapar las SR, con el beneficio de la reducción de fondo que genera y sin pérdida de señal.

En la Sección 5.2.1 se discutieron las dos posibles cadenas de decaimientos de los gluinos, dependiendo de las diferencias de masas con los neutralinos NLSP. Si $\Delta m_{\tilde{g}-\widetilde{\chi}_{1}^{0}}$ es pequeña, lo que se conoce como escenario comprimido, el gluino decae directamente al neutralino generando una cadena de decaimiento corta, con baja multiplicidad de jets, fotones muy energéticos y alto $E_{\mathrm{T}}^{\text {miss }}$, esto último debido a la alta masa del $\widetilde{\chi}_{1}^{0}$ y la correspondiente energía transferida al gravitino. Por otro lado, si $\Delta m_{\tilde{g}-\widetilde{\chi}_{1}^{0}}$ es grande, el gluino tiene la posibilidad de decaer en un paso intermedio a charginos (que a su vez decaen a los neutralinos), generando una cadena larga con alta multiplicidad de jets y por ende una gran actividad hadrónica, pero moderado $E_{\mathrm{T}}^{\text {miss }}$ y fotones menos energéticos. Esta notoria diferencia que surge dependiendo de la región del espacio observable de señal que se analiza, sugiere la construcción de dos regiones de señal, SRH para la cadena corta y SRL para la cadena larga. Por otro lado, debido a que las masas de los gluinos y neutralinos analizadas son muy grandes, llegando hasta $2.6 \mathrm{TeV}$ (véase la grid de señal en la Figura 5.4), los estados finales producidos por diferencias de masas intermedias quedan sub representados por sólo dos regiones de señal, por lo que se agrega una tercera región, SRM, dirigida exclusivamente a mejorar la selección en los puntos intermedios entre las otras dos regiones. En la Figura 5.6 se muestra un esquema de las tres regiones de señal en el plano formado por las masas del gluino y el neutralino NLSP (los límites de las regiones en la figura no son estrictos, existiendo un solapamiento parcial entre ellas). Se agregan también los nueve puntos de señal utilizados para realizar la presente optimización, para SRL: $(2000,250),(2200,250)$ y $(2400,250)$, para SRM: $(2000$, 1050), (2200, 1050) y $(2400,1050)$, y finalmente para SRH: $(2000,1950),(2200$, $2150)$ y $(2400,2350)$, donde siempre se escribe $\left(m_{\tilde{g}}, m_{\widetilde{\chi}_{1}^{0}}\right)$. Se eligen estos puntos en base a límites anteriores a las masas de las partículas y una sensibilidad "a priori" esperada.

A continuación, se hacen estudios sabiendo que a medida que la masa del $\widetilde{\chi}_{1}^{0}$ crece, la energía disponible para el fotón también lo hace, permitiendo incrementar el requerimiento en el $p_{\mathrm{T}}$ del fotón para SRM y SRH. El número de jets en el estado final es la variable principal que define las cadenas corta y larga, por lo que $\mathrm{SRH}$ (cadena corta) tendrá un requerimiento menor en el número de jets que SRL 


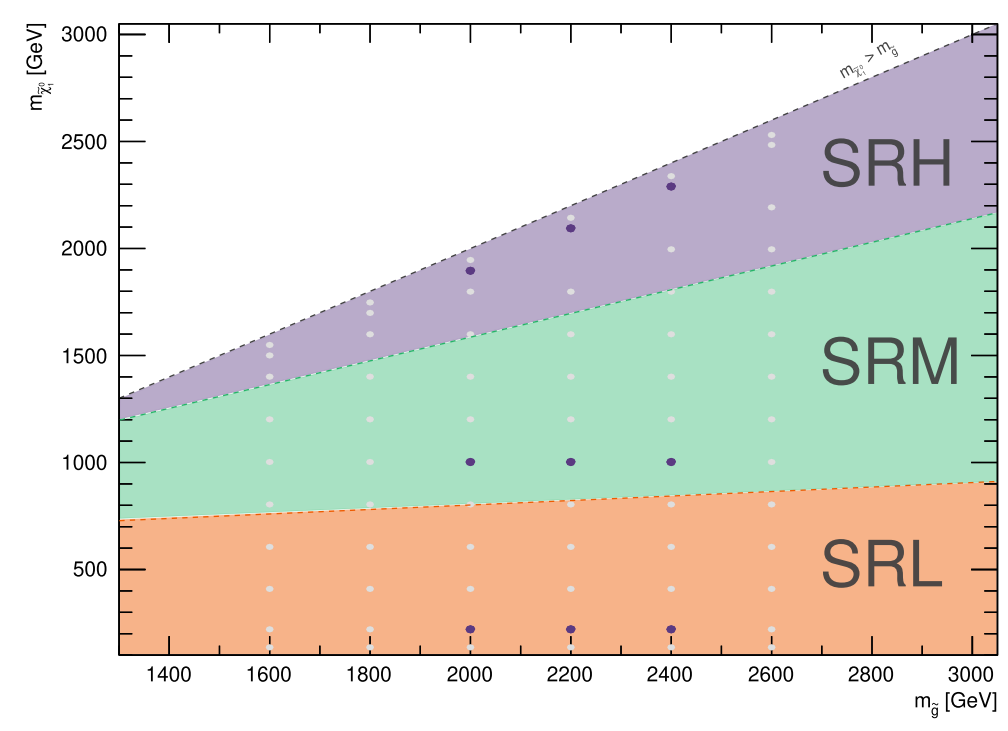

Figura 5.6: Esquema de las regiones de señal SRL, SRM y SRH, en el plano de las masas del gluino y el neutralino NLSP. Los puntos representan la grid de señal generada y, de esos, los nueve puntos oscuros resaltan los utilizados para realizar la optimización de las SR.

(cadena larga). Las distribuciones de $p_{\mathrm{T}}$ para el fotón leading y para el número de jets, para los modelos con $m_{\tilde{g}}=2200$ y $m_{\widetilde{\chi}_{1}^{0}}=250,1050$ y 1950 , se muestran en la Figura 5.7, de donde se desprende que SRM presenta multiplicidades de jets similares a las de SRL, pero mayores a SRH, y en los tres casos se observa el aumento correspondientemente esperado en el $p_{\mathrm{T}}$ de los fotones.
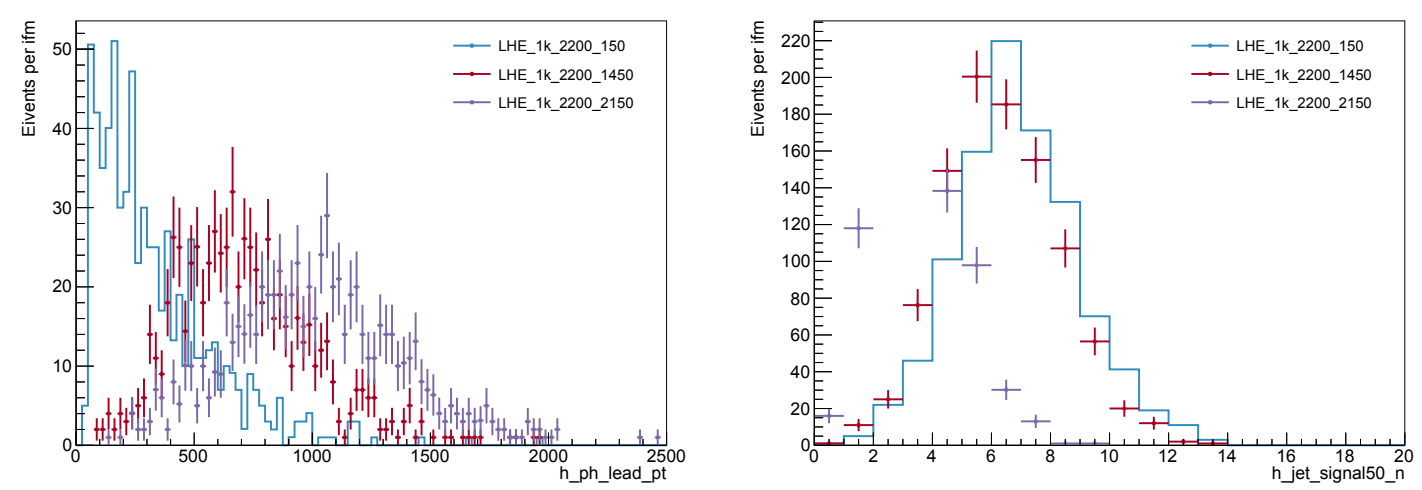

Figura 5.7: Distribuciones de $p_{\mathrm{T}}$ para el fotón leading (izquierda) y número de jets (derecha), para los modelos con $m_{\tilde{g}}=2200$ y $m_{\widetilde{\chi}_{1}^{0}}=250,1050$ y 2150 .

En eventos caracterizados por un $E_{\mathrm{T}}^{\text {miss }}$ falso, el vector de momento transversal perdido tiende a estar alineado con el fotón o con uno de los jets mal reconstruidos. Al mismo tiempo, en los eventos de SUSY considerados, se espera que esta distribución sea aleatoria, por lo que una selección basada en la separación angular entre estos objetos y el vector de $E_{\mathrm{T}}^{\text {miss }}\left(\Delta \phi\left(\right.\right.$ jet, $\left.E_{\mathrm{T}}^{\text {miss }}\right)$ y $\left.\Delta \phi\left(\gamma, E_{\mathrm{T}}^{\text {miss }}\right)\right)$ proporciona una supresión de estos procesos de fondo. Las distribuciones para $\Delta \phi$ (jet, $E_{\mathrm{T}}^{\text {miss }}$ ) y $\Delta \phi\left(\gamma, E_{\mathrm{T}}^{\mathrm{miss}}\right)$ con una preselección mínima se muestran en la Figura 5.8 para los 
fondos y la señal, normalizados a $139 \mathrm{fb}^{-1}$.
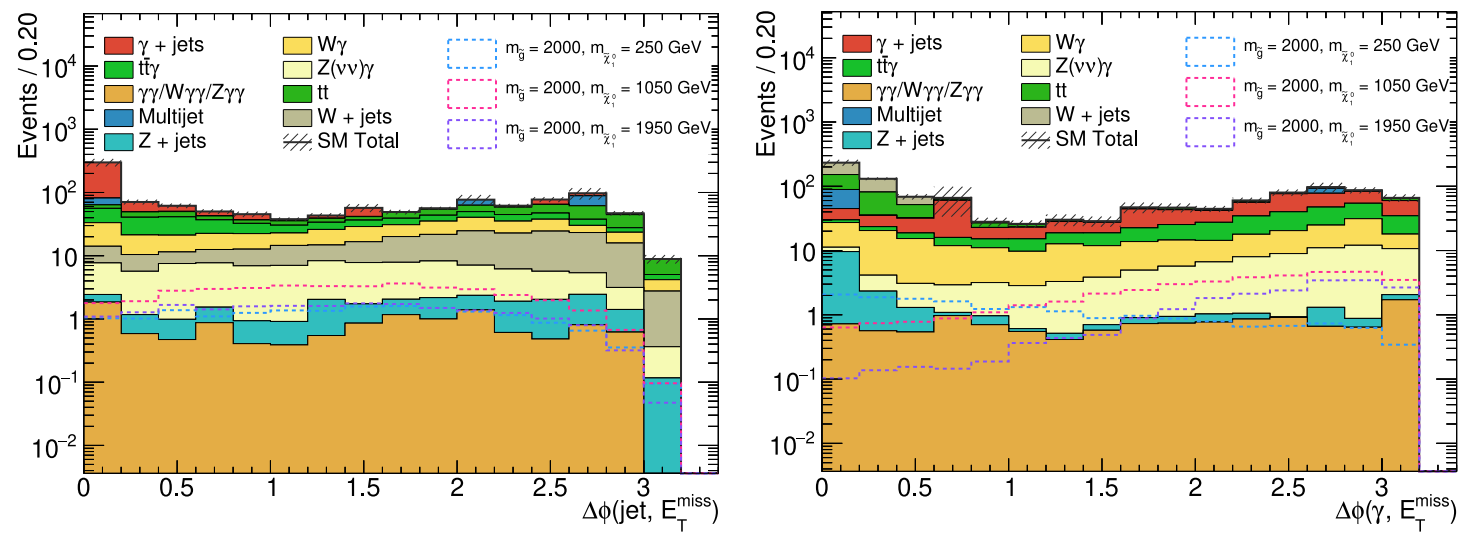

Figura 5.8: Distribuciones de $\Delta \phi\left(\right.$ jet, $\left.E_{\mathrm{T}}^{\text {miss }}\right)$ (izquierda) y $\Delta \phi\left(\gamma, E_{\mathrm{T}}^{\text {miss }}\right)$ (derecha), para los fondos del SM y para los modelos con $m_{\tilde{g}}=2000$ y $m_{\widetilde{\chi}_{1}^{0}}=250,1050$ y 1950 .

Antes de pasar a las variables discriminatorias más importantes que presenta esta búsqueda relacionadas con la escala de energía del evento, se presenta la variable de forma $R_{\mathrm{T}}^{4}$, relacionada con la multiplicidad de los jets, que agrega una reducción de fondo adicional y está definida como:

$$
R_{\mathrm{T}}^{4}=\frac{\sum_{i=1}^{4} p_{\mathrm{T}}^{\text {jet }_{i}}}{\sum_{\text {jets }} p_{\mathrm{T}}^{\text {jet }}},
$$

donde el 4 representa el número de jets elegido. Variables similares se pueden definir para cualquier $N_{\text {jets }}$, pero se encontró que para baja multiplicidad, la correspondiente variable no discrimina señal de fondo. Vale aclarar que esta variable sólo puede aplicarse a regiones con al menos cuatro jets, es decir SRL y SRM en la presente búsqueda. Una distribución de esta variable para una mínima selección se muestra en la Figura 5.9. Se espera que la señal tenga más de cuatro jets, con la energía distribuida en la multiplicidad de los jets producidos, mientras que los fondos del SM tienen en general la energía concentrada en sus cuatro jets principales.

Por la presencia de los $\widetilde{G}$ LSP en el estado final, se espera que la señal presente de moderado a alto $E_{\mathrm{T}}^{\text {miss }}$, permitiendo lograr una buena discriminación señal/fondo, ya que los procesos del SM, como los de QCD por ejemplo, producen bajo $E_{\mathrm{T}}^{\text {miss }}$ en comparación. En la Figura 5.10 se muestra una distribución de $E_{\mathrm{T}}^{\text {miss }}$ para una selección mínima, donde se aprecia que con un corte en $200 \mathrm{GeV}$ ya se produce una reducción de fondo de varios órdenes de magnitud. Al mismo tiempo, a medida que crece la masa del $\widetilde{\chi}_{1}^{0}$, el requerimiento en esta variable puede hacerse más alto y aumentar aún más la reducción de fondo.

Debido a la escala de energía que presentan los eventos buscados, iniciados con gluinos de alta masa y estados finales con alto $E_{\mathrm{T}}^{\text {miss }}$, un fotón energético y mucha actividad hadrónica, se estudiaron dos variables que son una medida de dicha escala. En primer lugar se define la variable $H_{\mathrm{T}}$, como la suma escalar del momento transversal de todos los jets de señal y del fotón principal en el evento (recordar que se aplica un veto de leptones): 


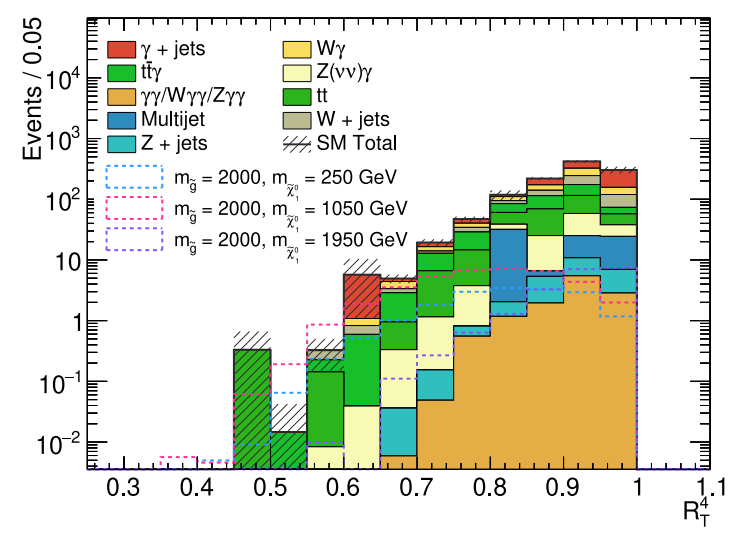

Figura 5.9: Distribución de $R_{\mathrm{T}}^{4}$ para los fondos del SM y los modelos con $m_{\tilde{g}}=2000$ y $m_{\widetilde{\chi}_{1}^{0}}=250,1050$ y 1950 .



Figura 5.10: Distribución de $E_{\mathrm{T}}^{\text {miss }}$ para los fondos del SM y para los modelos con $m_{\tilde{g}}=2000$ y $m_{\widetilde{\chi}_{1}^{0}}=250,1050$ y 1950 .

$$
H_{\mathrm{T}}=p_{\mathrm{T}}^{\text {leading }-\gamma}+\sum_{\text {jets }} p_{\mathrm{T}}^{\text {jet }}
$$

La otra variable, $m_{\text {eff }}$, la llamada masa efectiva del evento (utilizada extensivamente en estudios anteriores), está fuertemente correlacionada con $H_{\mathrm{T}}$ siendo definida como:

$$
m_{\mathrm{eff}}=H_{\mathrm{T}}+E_{\mathrm{T}}^{\mathrm{miss}}
$$

Ambas variables presentan un alto poder discriminatorio, como se observa en las distribuciones presentadas en la Figura 5.11, para una selección mínima. Sin embargo, debido a la fuerte correlación entre ambas variables y a su vez entre $m_{\text {eff }}$ y $E_{\mathrm{T}}^{\text {miss }}$, se prefirió utilizar $H_{\mathrm{T}}$ por sobre $m_{\mathrm{eff}}$, para permitir una mayor independencia y granularidad en los posibles cortes. Más adelante en la sección, se vuelve sobre esta decisión en la discusión de la significancia total esperada y se muestra la ventaja considerable lograda de esta forma. En las Figuras 5.12, 5.13 y 5.14 se muestra la significancia como función de $H_{\mathrm{T}}$ y de $E_{\mathrm{T}}^{\text {miss }}$ para determinar la selección final de 
SRL, SRM y SRH para un punto de señal representativo de cada región. Otras variables discriminatorias como la significancia en $E_{\mathrm{T}}^{\text {miss }}\left(E_{\mathrm{T}}^{\text {miss }} / \sqrt{H_{\mathrm{T}}}\right)$, o la llamada masa transversa, $m_{\mathrm{T}}\left(\sqrt{2 E_{\mathrm{T}} E_{\mathrm{T}}^{\text {miss }}}\left[1-\cos \left(\Delta \phi\left(\gamma, E_{\mathrm{T}}^{\text {miss }}\right)\right)\right]\right)$, fueron estudiadas pero los resultados indicaron que no aportan sensibilidad adicional para este análisis.
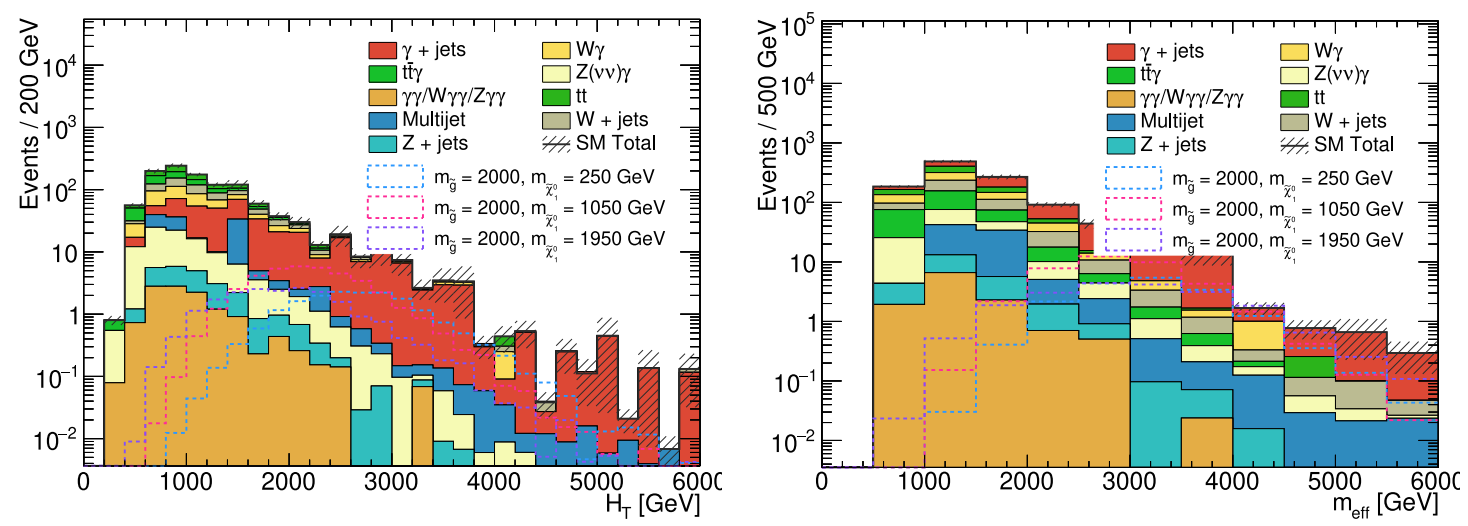

Figura 5.11: Distribuciones de $H_{\mathrm{T}}$ (izquierda) y $m_{\text {eff }}$ (derecha), para los fondos del SM y para los modelos con $m_{\tilde{g}}=2000$ y $m_{\widetilde{\chi}_{1}^{0}}=250,1050$ y 1950 .

\section{Definición de las regiones de señal}

En base a los estudios presentados y a un proceso iterativo de optimización de cortes para maximizar la sensibilidad, se definen los criterios de selección óptimos para SRL, SRM y SRH, que se resumen en la Tabla 5.7.

\begin{tabular}{|l|r|r|r|}
\hline & SRL & SRM & SRH \\
\hline$N_{\text {fotones }}$ & $\geq 1$ & $\geq 1$ & $\geq 1$ \\
$p_{\mathrm{T}}^{\text {lading- }}$ & $>145 \mathrm{GeV}$ & $>300 \mathrm{GeV}$ & $>400 \mathrm{GeV}$ \\
$N_{\text {leptones }}$ & 0 & 0 & 0 \\
$N_{\text {jets }}$ & $\geq 5$ & $\geq 5$ & $\geq 3$ \\
$\Delta \phi\left(\right.$ jet, $\left.E_{\mathrm{T}}^{\text {miss }}\right)$ & $>0.4$ & $>0.4$ & $>0.4$ \\
$\Delta \phi\left(\gamma, E_{\mathrm{T}}^{\text {miss }}\right)$ & $>0.4$ & $>0.4$ & $>0.4$ \\
$E_{\mathrm{T}}^{\text {miss }}$ & $>250 \mathrm{GeV}$ & $>300 \mathrm{GeV}$ & $>600 \mathrm{GeV}$ \\
$H_{\mathrm{T}}$ & $>2000 \mathrm{GeV}$ & $>1600 \mathrm{GeV}$ & $>1600 \mathrm{GeV}$ \\
$R_{\mathrm{T}}^{4}$ & $<0.90$ & $<0.90$ & - \\
\hline
\end{tabular}

Tabla 5.7: Selección para las regiones SRL, SRM y SRH.

La significancia de descubrimiento esperada para todos los puntos de la grid de señal, eligiendo el valor más alto de cada una de las tres regiones, se muestra en la Figura 5.15, para regiones que utilizan un requerimiento en $m_{\text {eff }}$ (según se trabajó en un análisis preliminar [2]), equivalente al optimizado en las SR de $H_{\mathrm{T}}$, y para las regiones descriptas en la tabla anterior $\left(\operatorname{con} H_{\mathrm{T}}\right.$ ). Se puede apreciar la ventaja de requerir en $H_{\mathrm{T}}$ por sobre $m_{\text {eff }}$, extendiendo los límites de descubrimiento esperados (a 3 o $5 \sigma$ ) entre 50 y $100 \mathrm{GeV}$, dependiendo del punto de la grid. Es importante aclarar que estos resultados de significancias son considerados preliminares, ya que se obtuvieron inicialmente durante el proceso de optimización considerando sólo muestras de $\mathrm{MC}$, con normalizaciones preliminares y sin un tratamiento completo 


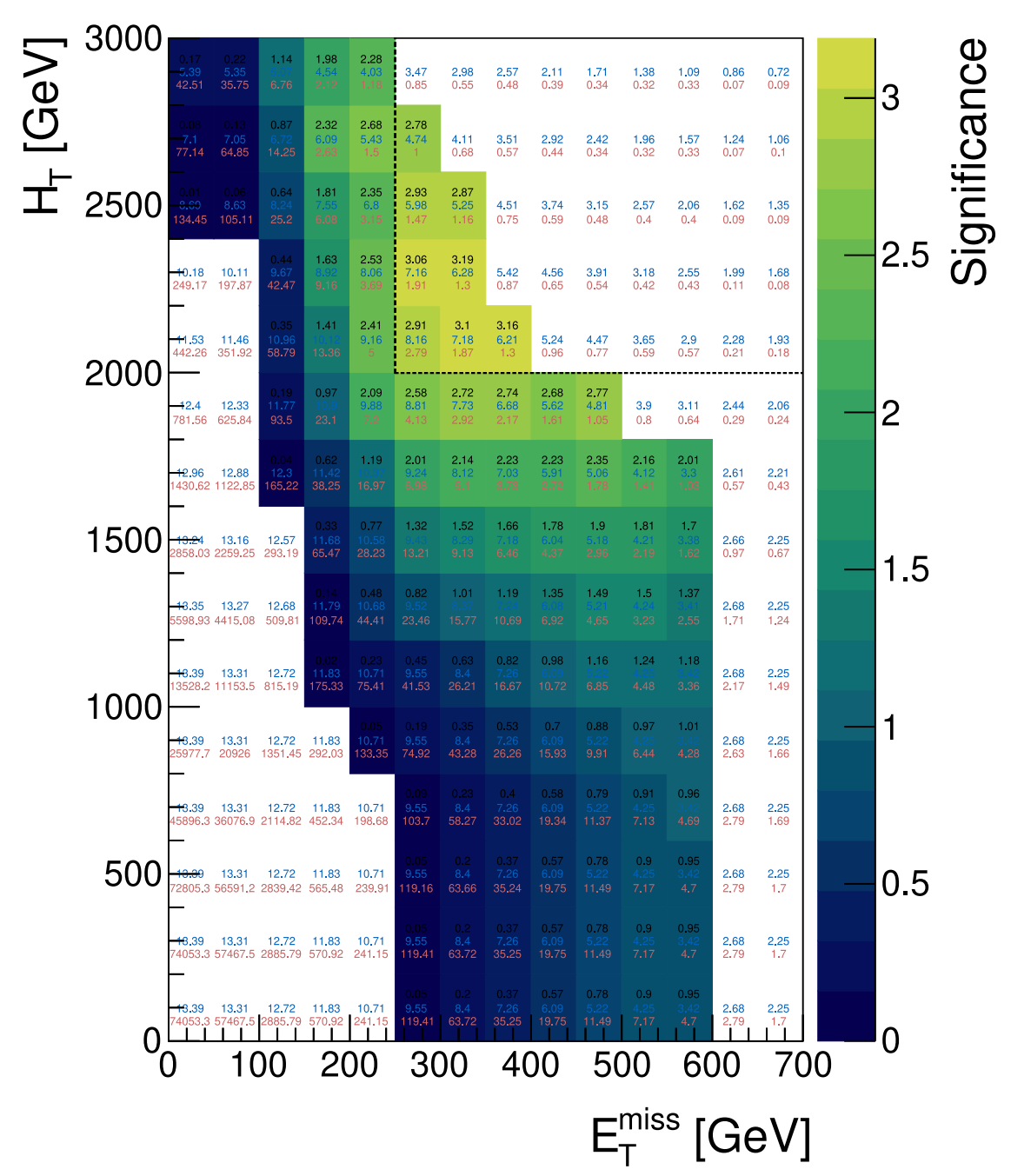

Figura 5.12: Significancia de descubrimiento como función de $H_{\mathrm{T}}$ y de $E_{\mathrm{T}}^{\text {miss }}$ para el punto de señal con $m_{\tilde{g}}=2000$ y $m_{\widetilde{\chi}_{1}^{0}}=250$ para SRL, para una luminosidad integrada de $139 \mathrm{fb}^{-1}$. La significancia tiene en cuenta $30 \%$ de incerteza para el fondo, y se iguala a cero si el número de eventos de señal es menor que tres o el número de eventos de fondo es menor que uno. Se agregan en azul y en rojo el número de eventos de señal y fondo respectivamente.

de las incertezas. Los límites de descubrimiento se presentan, junto al resto de los resultados, en el próximo capítulo.

\subsubsection{Regiones de control y validación}

Para la presente búsqueda se definen tres regiones de control, que se denominan CRQ, CRW y CRT, y se utilizan para determinar la normalización de las muestras simuladas para los eventos QCD $\gamma+$ jets, $W \gamma$ y $t \bar{t} \gamma$, respectivamente. Como se mencionó en secciones anteriores, las CR se definen para que los eventos incluidos provengan de manera dominante del proceso de fondo elegido y, al mismo tiempo, con una contaminación de señal nula o despreciable.

La selección dedicada para CRQ se define desde las SR, pero aplicando un re- 


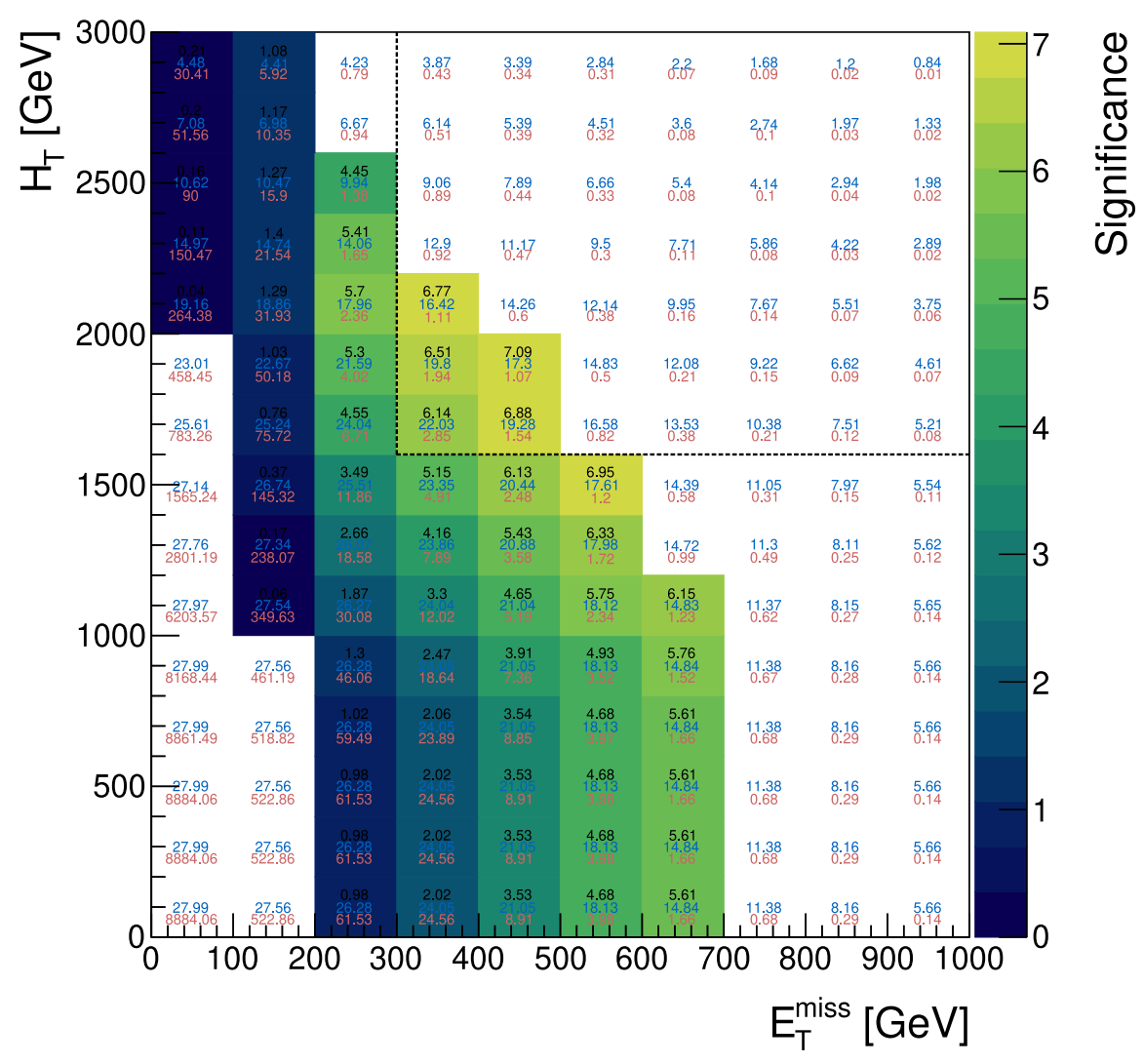

Figura 5.13: Significancia de descubrimiento como función de $H_{\mathrm{T}}$ y de $E_{\mathrm{T}}^{\text {miss }}$ para el punto de señal con $m_{\tilde{g}}=2000$ y $m_{\widetilde{\chi}_{1}^{0}}=1050$ para SRM, para una luminosidad integrada de $139 \mathrm{fb}^{-1}$. La significancia tiene en cuenta $30 \%$ de incerteza para el fondo, y se iguala a cero si el número de eventos de señal es menor que tres o el número de eventos de fondo es menor que uno. Se agregan en azul y en rojo el número de eventos de señal y fondo respectivamente.

quisito menor en $E_{\mathrm{T}}^{\text {miss }}\left(>100 \mathrm{GeV}\right.$ ), una selección similar en $H_{\mathrm{T}}$, e invirtiendo el requerimiento en $\Delta \phi\left(\right.$ jet, $\left.E_{\mathrm{T}}^{\text {miss }}\right)$ para aumentar el tamaño de $\gamma+$ jets en la muestra de control, al mismo tiempo que se la vuelve ortogonal a las SR. Es oportuno recordar aquí que este fondo ingresa a las distintas regiones producto de falso $E_{\mathrm{T}}^{\text {miss }}$ proveniente de una mala reconstrucción de los jets, y por lo tanto la variable angular $\Delta \phi$ invertida es por demás relevante, como se discutió en la sección de optimización.

Las regiones CRW y CRT se definen ortogonales a las SR requiriendo un leptón. A su vez, CRW requiere un fotón, $100 \mathrm{GeV}<E_{\mathrm{T}}^{\text {miss }}<200 \mathrm{GeV}$ y se relaja el corte en $H_{\mathrm{T}}$ para aumentar la estadística. Se aplica un veto de $b$-jets para reducir la contaminación de $t \bar{t} \gamma$. En el caso de CRT, la muestra se define requiriendo un fotón, jets, $50 \mathrm{GeV}<E_{\mathrm{T}}^{\text {miss }}<200 \mathrm{GeV}$, y el mismo requerimiento en $H_{\mathrm{T}}$ que CRW. Se necesitan al menos dos jets etiquetados como $b$-jets en la CRT para aumentar la población de eventos $t \bar{t} \gamma$. En ambos casos se aplican requisitos menos estrictos en $E_{\mathrm{T}}^{\text {miss }}$ para aumentar la estadística. No se aplica ningún requisito en $R_{\mathrm{T}}^{4}$ por la misma razón. A su vez, se aplica un requisito máximo en $E_{\mathrm{T}}^{\text {miss }}$ para reducir la contaminación de la señal.

Los criterios de selección de las CR asociadas a las SR se presentan en la Tabla 


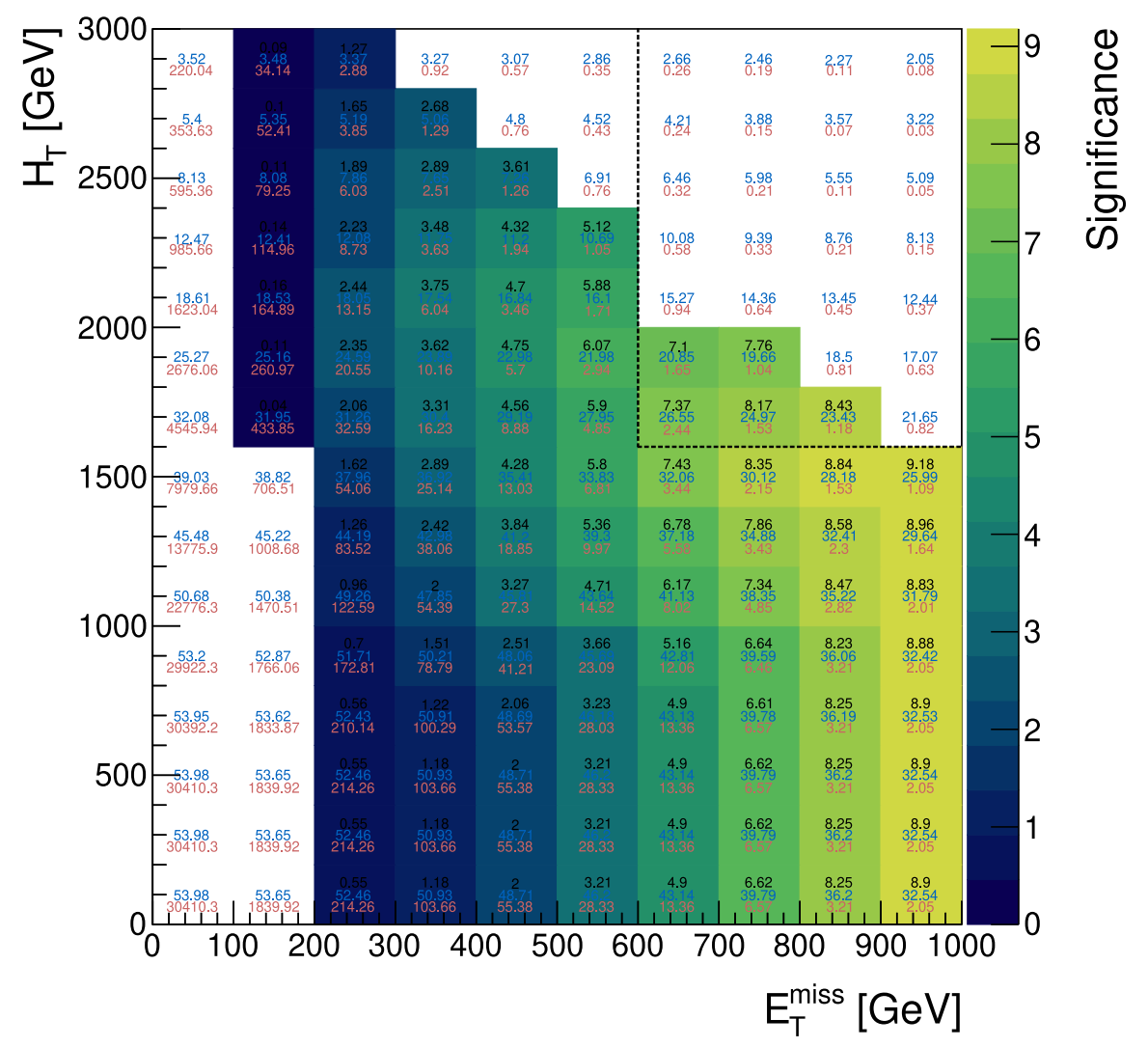

Figura 5.14: Significancia de descubrimiento como función de $H_{\mathrm{T}}$ y de $E_{\mathrm{T}}^{\text {miss }}$ para el punto de señal con $m_{\tilde{g}}=2000$ y $m_{\widetilde{\chi}_{1}^{0}}=1950$ para $S R H$, para una luminosidad integrada de $139 \mathrm{fb}^{-1}$. La significancia tiene en cuenta $30 \%$ de incerteza para el fondo, y se iguala a cero si el número de eventos de señal es menor que tres o el número de eventos de fondo es menor que uno. Se agregan en azul y en rojo el número de eventos de señal y fondo respectivamente.
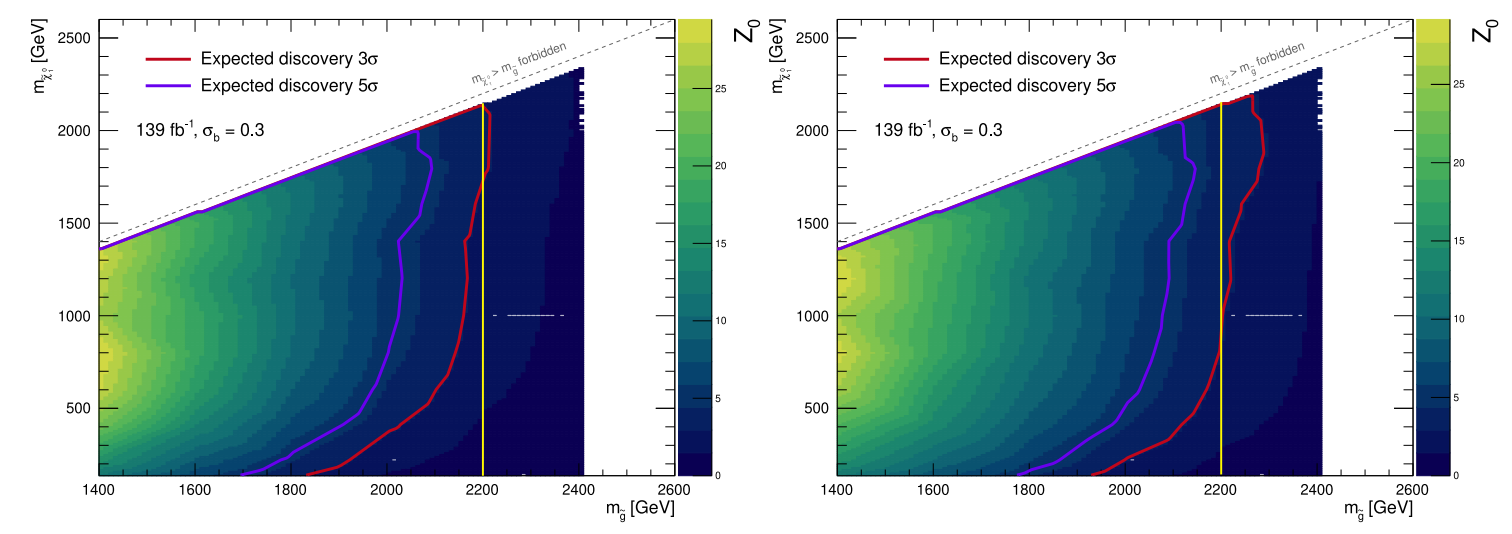

Figura 5.15: Significancia combinada de descubrimiento esperada para todos los puntos de la grid de señal en el plano $\left(m_{\tilde{g}}, m_{\widetilde{\chi}_{1}^{0}}\right)$. En cada punto se toma la región con mayor significancia. Se agregan los contornos de $3 \sigma$ y $5 \sigma$. A la izquierda se muestra para las regiones que usan $m_{\text {eff }}$, y a la derecha las que usan $H_{\mathrm{T}}$. Se observa una mejora de entre 50 y $100 \mathrm{GeV}$ al utilizar $H_{\mathrm{T}}$. 
5.8. La contaminación de señal en las regiones de control así definidas se muestra en la Figura 5.16, donde se puede ver que, para la gran mayoría de los puntos de la grid de señal, las CR tienen menos de $1 \%$ de contaminación, a excepción de algunos puntos para bajas masas de gluinos y neutralinos (que de todas formas ya han sido excluidos por búsquedas anteriores [2]).

\begin{tabular}{|l|r|r|r|}
\hline & CRQ & CRW & CRT \\
\hline$N_{\text {fotones }}$ & $\geq 1$ & $\geq 1$ & $\geq 1$ \\
$p_{\mathrm{T}}^{\text {leading- }}$ & $>145 \mathrm{GeV}$ & $>145 \mathrm{GeV}$ & $>145 \mathrm{GeV}$ \\
$N_{\text {leptones }}$ & 0 & $\geq 1$ & $\geq 1$ \\
$N_{\text {jets }}$ & $\geq 3$ & $\geq 1$ & $\geq 2$ \\
$N_{b-\text { jets }}$ & - & 0 & $\geq 2$ \\
$\Delta \phi\left(\right.$ jet, $\left.E_{\mathrm{T}}^{\text {miss }}\right)$ & $<0.4$ & $>0.4$ & $>0.4$ \\
$\Delta \phi\left(\gamma, E_{\mathrm{T}}^{\text {miss }}\right)$ & $>0.4$ & - & - \\
$E_{\mathrm{T}}^{\text {miss }}$ & $>100 \mathrm{GeV}$ & {$[100,200] \mathrm{GeV}$} & {$[50,200] \mathrm{GeV}$} \\
$H_{\mathrm{T}}$ & $>1600 \mathrm{GeV}$ & $>400 \mathrm{GeV}$ & $>400 \mathrm{GeV}$ \\
\hline
\end{tabular}

Tabla 5.8: Criterios de selección (basados en los objetos de señal) para las regiones de control, que son las mismas para SRL, SRM y SRH.
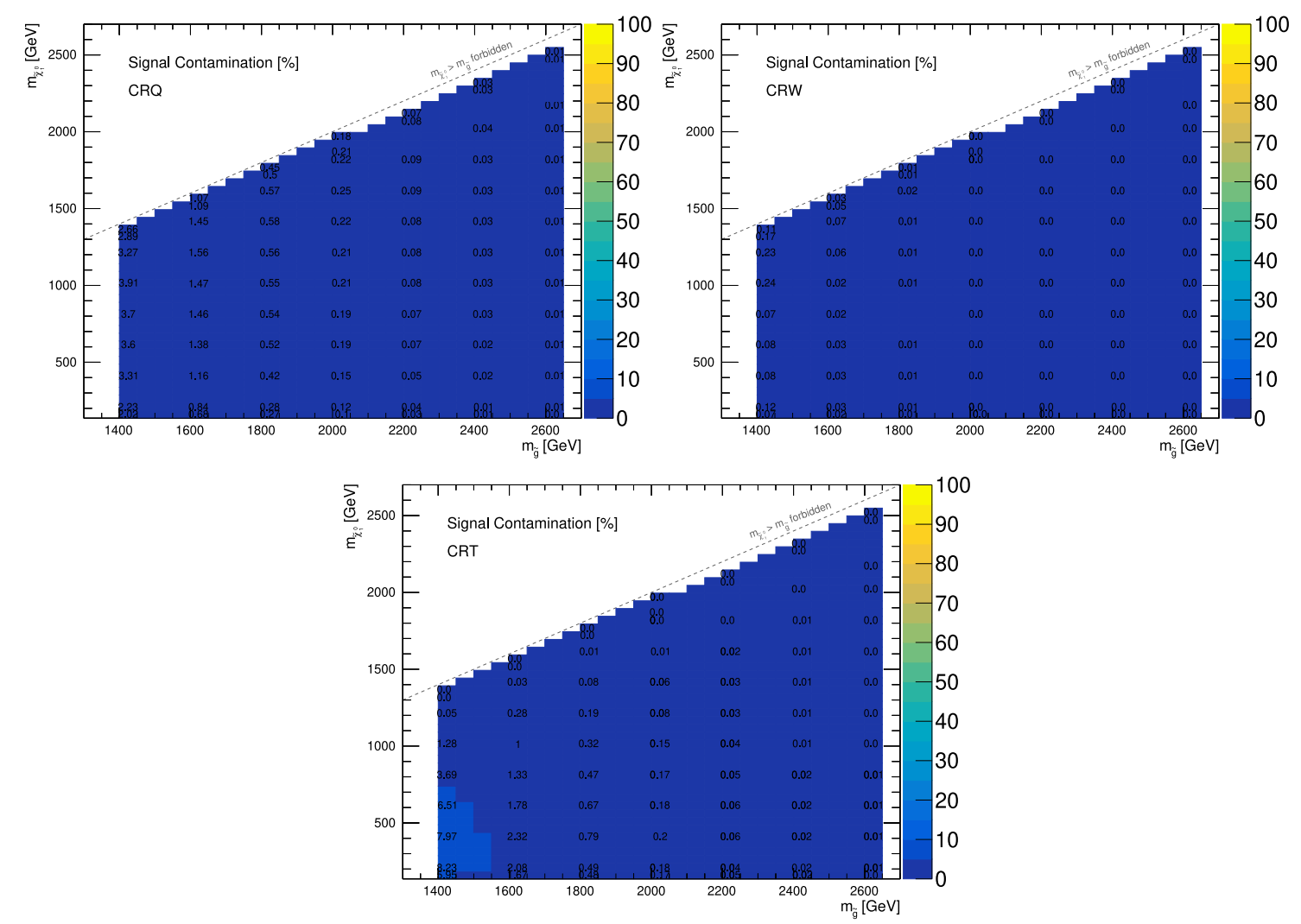

Figura 5.16: Contaminación de señal (en \%), definida como $\left(100 \cdot \frac{n_{s}}{n_{s}+n_{b}}\right)$, en CRQ, CRW y CRT para todos los puntos de la grid de señal en el plano $\left(m_{\tilde{g}}, m_{\widetilde{\chi}_{1}^{0}}\right)$.

Finalmente se definen las regiones de validación (VR) utilizadas para verificar que el procedimiento de extrapolación de los fondos desde las CR, funciona correctamente. Estas VR se encuentran en una región intermedia entre las CR y las SR, 
siendo cinemáticamente similares a las regiones de señal, pero con uno o más criterios invertidos o modificados para reducir una posible contaminación de la señal y mantener la ortogonalidad con las SR. En la presente búsqueda se definen dos conjuntos de VR. El primero está destinado a validar el fondo de $\gamma+$ jets y el otro para los fondos de $W \gamma \mathrm{y} t \bar{t} \gamma$.

Para el caso de $\gamma+$ jets y CRQ, se definen tres grupos de regiones. VRQ recupera el corte en $\Delta \phi$ (jet, $\left.E_{\mathrm{T}}^{\text {miss }}\right)$ de las $\mathrm{SR}$, y se mantiene ortogonal con un requerimiento de $E_{\mathrm{T}}^{\text {miss }}$ estrictamente menor. Al tener un bajo requerimiento en la multiplicidad de los jets y en el $p_{\mathrm{T}}$ del fotón, esta región intenta ser lo más inclusiva posible. Luego, se definen las VRML ( 1 y 2 ) y las VRMH (l y $\left.\begin{array}{ll}1 & \text { y }\end{array}\right)$, que apuntan cada una a una cadena de decaimiento del gluino. Las L requieren mayor multiplicidad de jets y un corte en $R_{\mathrm{T}}^{4}$, como lo hacen SRL y SRM, mientras que las $\mathrm{H}$ apuntan a la cadena corta para validar SRH. La ortogonalidad viene nuevamente del requerimiento en $E_{\mathrm{T}}^{\text {miss }}$. Las diferencias entre las regiones con un 1 o un 2 aparecen sólo en el rango de $E_{\mathrm{T}}^{\text {miss }}$, y con esto se busca una validación lo más cercana posible a las regiones, a costa de un aumento en la contaminación de señal.

Las regiones VRL se definen para estar enriquecidas con los fondos $W \gamma$ y $t \bar{t} \gamma$. La ortogonalidad de estas cuatro regiones con las SR viene de la presencia de leptones. En particular, VRL4 invierte el corte en $\Delta \phi\left(\right.$ jet, $\left.E_{\mathrm{T}}^{\text {miss }}\right)$ para verificar en regiones angulares distintas tanto a las CR como a las SR. Las diferencias principales están en los requerimientos de $E_{\mathrm{T}}^{\text {miss }}$, donde VRL1 y VRL2 analizan las regiones con $E_{\mathrm{T}}^{\text {miss }}<200 \mathrm{GeV}$ mientras que las otras dos lo hacen para valores mayores. Diferentes requerimientos en $H_{\mathrm{T}}$ permiten validar aún más regiones de parámetros y aumentar la cobertura de la validación.

Un resumen de los diferentes criterios de selección se muestran en las Tablas 5.9 y 5.10. La contaminación de señal para cada una de las regiones de validación se muestra en la Figura 5.17. Se puede ver la baja contaminación en todas las regiones, donde solo se supera el $10 \%$ de contaminación en puntos de la grid que ya han sido excluidos por búsquedas anteriores.

\begin{tabular}{|c|c|c|c|}
\hline & VRQ & VRM1L (VRM2L) & VRM1H (VRM2H) \\
\hline$N_{\text {fotones }}$ & $\geq 1$ & $\geq 1$ & $\geq 1$ \\
\hline$p_{\mathrm{T}}^{\text {leading }-\gamma}$ & $>145 \mathrm{GeV}$ & $>145 \mathrm{GeV}$ & $>300 \mathrm{GeV}$ \\
\hline$N_{\text {leptones }}$ & 0 & 0 & 0 \\
\hline$N_{\text {jets }}$ & $\geq 3$ & $\geq 5$ & $\geq 3$ \\
\hline$\Delta \phi\left(\right.$ jet,$\left.E_{\mathrm{T}}^{\text {miss }}\right)$ & $>0.4$ & $>0.4$ & $>0.4$ \\
\hline$\Delta \phi\left(\gamma, E_{\mathrm{T}}^{\text {miss }}\right)$ & $>0.4$ & $>0.4$ & $>0.4$ \\
\hline$E_{\mathrm{T}}^{\text {miss }}$ & {$[100,200]$} & {$[100,200]([150,200])$} & {$[100,200]([150,200])$} \\
\hline$H_{\mathrm{T}}$ & $>1600$ & $>1600$ & $>1600$ \\
\hline$R_{T}^{4}$ & - & $<0.90$ & - \\
\hline
\end{tabular}

Tabla 5.9: Criterios de selección para las regiones de validación usadas para validar el fondo $\gamma+$ jets. 


\begin{tabular}{|l|r|r|r|r|}
\hline & VRL1 & VRL2 & VRL3 & VRL4 \\
\hline$N_{\text {fotones }}$ & $\geq 1$ & $\geq 1$ & $\geq 1$ & $\geq 1$ \\
$p_{\mathrm{T}}^{\text {leading- }}$ & $>145 \mathrm{GeV}$ & $>145 \mathrm{GeV}$ & $>145 \mathrm{GeV}$ & $>145 \mathrm{GeV}$ \\
$N_{\text {leptones }}$ & $\geq 1$ & $\geq 1$ & $\geq 1$ & $\geq 1$ \\
$N_{\text {jets }}$ & $\geq 2$ & $\geq 2$ & $\geq 2$ & $\geq 2$ \\
$\Delta \phi\left(\right.$ jet, $\left.E_{\mathrm{T}}^{\text {miss }}\right)$ & $>0.4$ & $>0.4$ & $>0.4$ & $<0.4$ \\
$E_{\mathrm{T}}^{\text {miss }}$ & {$[50,200] \mathrm{GeV}$} & {$[50,200] \mathrm{GeV}$} & $>200 \mathrm{GeV}$ & $>200 \mathrm{GeV}$ \\
$H_{\mathrm{T}}$ & $>800 \mathrm{GeV}$ & $>1300 \mathrm{GeV}$ & {$[600,1600] \mathrm{GeV}$} & $>1100 \mathrm{GeV}$ \\
\hline
\end{tabular}

Tabla 5.10: Criterios de selección para las regiones de validación usadas para validar los fondos $W \gamma \mathrm{y} t \bar{t} \gamma$. 

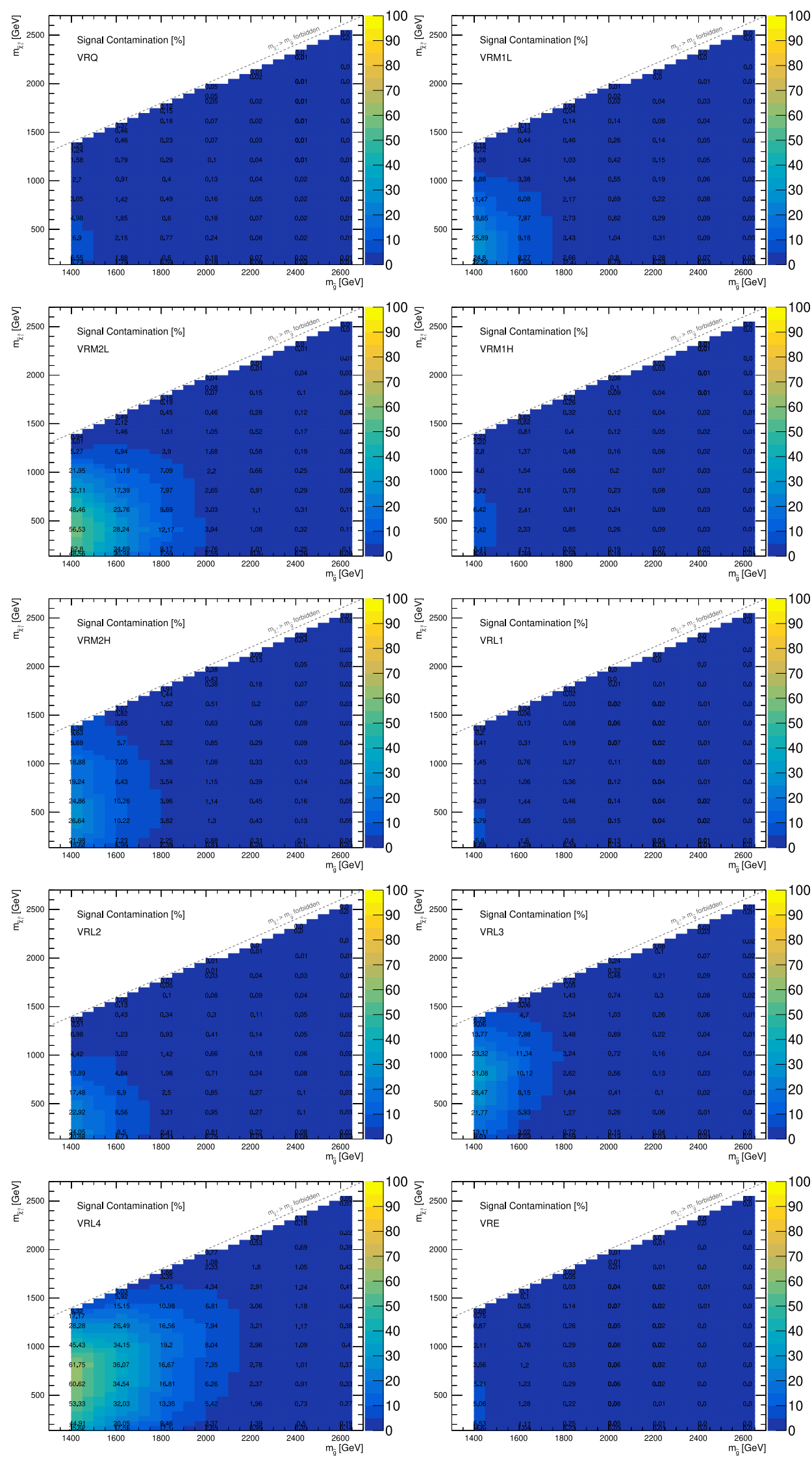

Figura 5.17: Contaminación de señal (en \%), definida como $100 \cdot \frac{n_{s}}{n_{s}+n_{b}}$, en las diferentes regiones de validación para todos los puntos de la grid de señal en el plano $\left(m_{\tilde{g}}, m_{\widetilde{\chi}_{1}^{0}}\right)$. 


\subsection{Estimación del fondo en base a técnicas de da- tos}

Los fondos con electrones y jets mal identificados como fotones, se determinan con las técnicas data-driven que se describen a continuación. Los jets pueden ser mal identificados como fotones (fotones falsos) si contienen un $\pi^{0}$ que lleva la mayor parte de la energía del jet y decae en un par de fotones colimados. Este fondo surge principalmente de los eventos QCD multijets, $\mathrm{W}+$ jets y $t \bar{t}$ semi-leptónicos. Como es extremadamente difícil modelar con precisión la tasa de identificación errónea de jets a fotones, se utiliza una determinación data-driven, el llamado método ABCD, que utiliza los diferentes perfiles de aislamiento esperados para fotones reales y falsos, tal como se describe en [146].

En los casos en que un electrón de alto $p_{\mathrm{T}}$ es identificado erróneamente como un fotón, se espera un fondo significativo de los procesos del SM como $W / Z+$ jets y eventos $t \bar{t}$. La tasa de electrones falseando un fotón se obtiene a partir del decaimiento $Z(\rightarrow e e)$, reconstruido como $e^{+} e^{-}$o $e \gamma[120]$, y se explica en más detalle en la Sección 5.4.2.

\subsubsection{Jets falseando fotones}

Los fotones falsos provenientes de jets surgen principalmente de los eventos QCD multijets, $\mathrm{W}+$ jets y eventos $t \bar{t}$ semi-leptónicos, y constituyen una fuente importante de fondo. El método implementado en este análisis es similar al utilizado en [147].

Como se mencionó antes, los jets pueden ser mal identificados como fotones (fotones falsos) si contienen un $\pi^{0}$, resultando en un objeto electromagnético indistinguible de un solo fotón altamente energético. Para hacer frente a los grandes fondos de jets, se aplican los criterios de identificación tight a los candidatos a fotones. Se espera que esta selección contenga fotones reales con una contaminación de jets moderada.

Se ha utilizado una determinación basada en datos, el llamado método ABCD, para determinar la tasa de identificación errónea de jets a fotones, que utiliza los diferentes perfiles de aislamiento esperados para fotones reales y falsos. La identificación offline tight es por diseño más estricta que el trigger de fotones usado para recolectar los datos, por lo que se encuentra que hay candidatos a fotones provenientes de jets que fallarán los criterios tight, pero satisfacen alguna identificación intermedia. Estos jets similares a fotones, de aquí en adelante llamados pseudo-fotones (o non-tight), se definen como aquellos que pasan la identificación loose pero fallan (al menos) uno de los requerimientos de selección tight siguientes: $w_{s 3}, F_{\text {side }}, \Delta E, E_{\text {ratio }}$, de similar manera al método descripto en la Sección 4.3. El método ABCD define una región de señal $A$ y tres regiones de control, a saber $B, C$ y $D$. Para este estudio se definen como [148]:

- Requerimientos comunes: $\mathrm{Al}$ menos un fotón de señal con $p_{\mathrm{T}}>145 \mathrm{GeV}$, $N_{\text {signal-jets }} \geq 2$ y $N_{\text {signal-leptons }}=0$.

- Región $A$ : fotones tight, $E_{\mathrm{T}}^{\text {iso }}<0 \mathrm{GeV}$ y $p_{\mathrm{T}}^{\text {iso }}<0.05 \mathrm{GeV}$.

- Región $B$ : fotones tight y $8 \mathrm{GeV}<E_{\mathrm{T}}^{\text {iso }}<80 \mathrm{GeV}$ o $0.15 \mathrm{GeV}<p_{\mathrm{T}}^{\text {iso }}<1 \mathrm{GeV}$. 
- Región $C$ : pseudo-fotones, $E_{\mathrm{T}}^{\text {iso }}<0 \mathrm{GeV}$ y $p_{\mathrm{T}}^{\text {iso }}<0.05 \mathrm{GeV}$.

- Región $D$ : pseudo-fotones y $8 \mathrm{GeV}<E_{\mathrm{T}}^{\text {iso }}<80 \mathrm{GeV}$ o $0.15 \mathrm{GeV}<p_{\mathrm{T}}^{\text {iso }}<$ $1 \mathrm{GeV}$.

donde $E_{\mathrm{T}}^{\text {iso }}$ y $p_{\mathrm{T}}^{\text {iso }}$ son las variables de aislamiento definidas en el apartado 3.1.3.

La Figura 5.18 muestra las cuatro regiones diferentes en función de $E_{\mathrm{T}}^{\text {iso }}$ o $p_{\mathrm{T}}^{\text {iso }}$, para el conjunto completo de datos de Run- 2 .
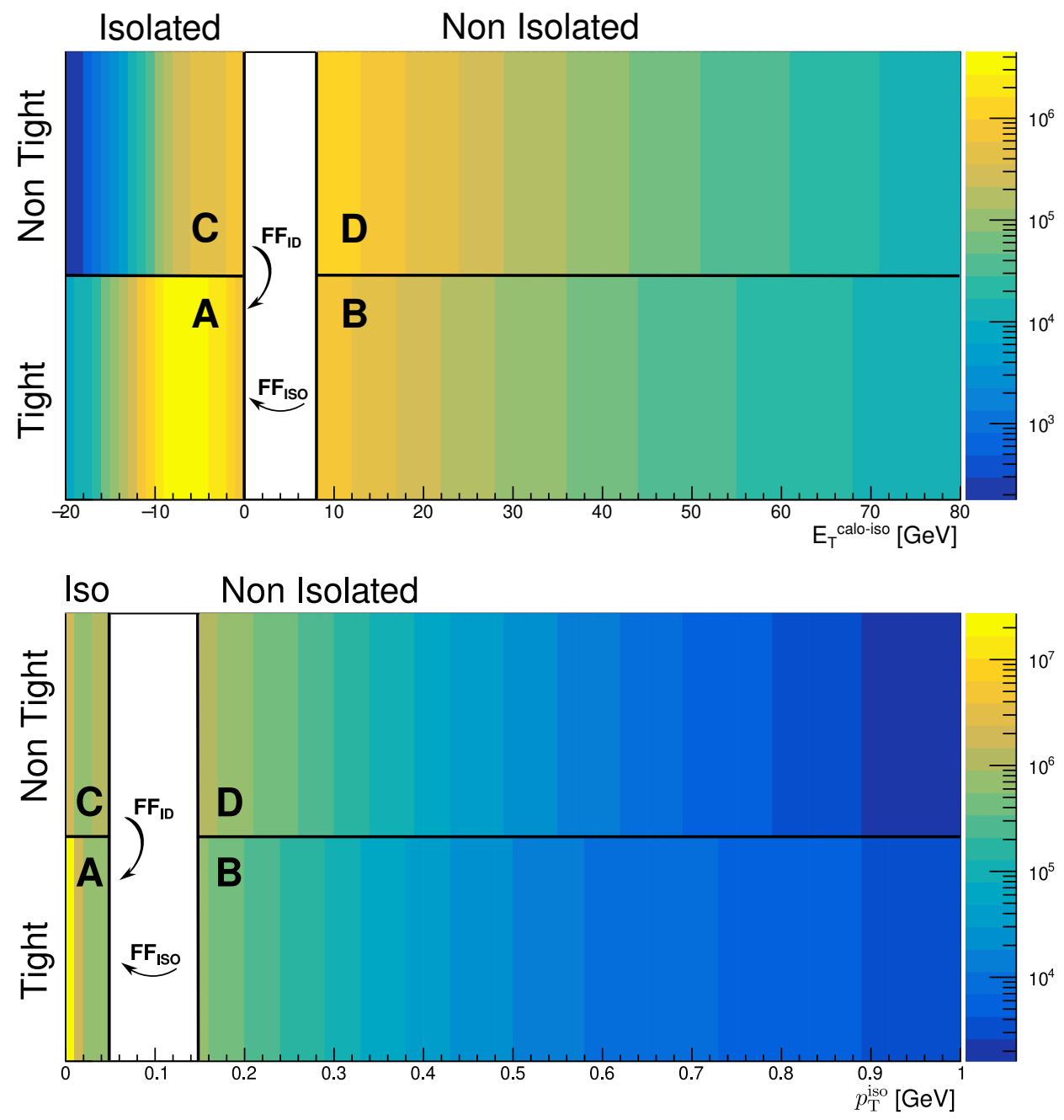

Figura 5.18: Plano bidimensional definido con el aislamiento calorimétrico (arriba) o aislamiento en las trazas (abajo) y la identificación tight/non-tight para las regiones $A, B, C$ y $D$, para el conjunto completo de datos de Run-2.

En $[147,46]$ se muestra la aplicación del método con la distribución del aislamiento para los candidatos tight y non-tight (normalizada a los candidatos tight no aislados). El acuerdo entre ambas distribuciones muestra cualitativamente la validez de la hipótesis del modelo original.

Suponiendo que no hay contaminación de señal en ninguna región de control, las regiones $B, C$ y $D$ sólo están compuestas de fondo $N_{(B, C, D)}=N_{(B, C, D)}^{b}$. Además, con la suposición adicional que no hay correlación entre el aislamiento y las variables de forma consideradas para la definición de los non-tight, la siguiente relación es válida: $N_{B}^{b} / N_{A}^{b}=N_{D}^{b} / N_{C}^{b}$. Por lo tanto, se pueden definir dos fakes factors $(F F)$ diferentes 
(mostrados esquemáticamente en la Figura 5.18). En particular, en este análisis se utiliza:

$$
F F_{\text {ISO }}=\frac{N_{C}}{N_{D}}
$$

Con lo que el número de fotones falsos provenientes de jets puede estimarse usando el FF como:

$$
N_{j \rightarrow \gamma}=N_{A}=F F_{\text {ISO }} N_{B}
$$

Teniendo en cuenta la contaminación de fotones de señal en las regiones de control, la relación se vuelve:

$$
N_{j \rightarrow \gamma}=\frac{N_{B}-N_{B}^{s}}{N_{D}-N_{D}^{s}}\left(N_{C}-N_{C}^{s}\right)
$$

donde $N_{(B, C, D)}^{s}$ es el número de fotones reales en cada región y se estima usando muestras MC a nivel generador (MC TRUTH) de fotones.

La presencia de una correlación residual del fondo en las cuatro regiones, que puede manifestarse como una diferencia en las distribuciones de fondo para las regiones tight y non-tight, podría tenerse en cuenta calculando [149, 46]:

$$
R=\frac{N_{A}^{b} N_{D}^{b}}{N_{B}^{b} N_{C}^{b}} \neq 1
$$

Sin embargo, como $R$ no se puede obtener en los datos, ya que esto implicaría utilizar los datos de la región de señal $(A)$ a los que solo se accede para la obtención de resultados finales y no pueden utilizarse en esta etapa del análisis, se calcula un parámetro equivalente:

$$
R^{\prime}=\frac{N_{A}^{\prime} N_{D}^{\prime}}{N_{B}^{\prime} N_{C}^{\prime}}
$$

con la siguiente definición para cada región primada:

- región $A^{\prime}$ : fotones tight y $9 \mathrm{GeV}<E_{\mathrm{T}}^{\text {iso }}<15 \mathrm{GeV}$ o $0.2 \mathrm{GeV}<p_{\mathrm{T}}^{\text {iso }}<0.3 \mathrm{GeV}$.

- región $B^{\prime}$ : fotones tight y $15 \mathrm{GeV}<E_{\mathrm{T}}^{\text {iso }}<80 \mathrm{GeV}$ o $0.3 \mathrm{GeV}<p_{\mathrm{T}}^{\text {iso }}<1 \mathrm{GeV}$.

- región $C^{\prime}$ : pseudo-fotones y $9 \mathrm{GeV}<E_{\mathrm{T}}^{\text {iso }}<15 \mathrm{GeV}$ o $0.2 \mathrm{GeV}<p_{\mathrm{T}}^{\text {iso }}<$ $0.3 \mathrm{GeV}$.

- región $D^{\prime}$ : pseudo-fotones y $15 \mathrm{GeV}<E_{\mathrm{T}}^{\text {iso }}<80 \mathrm{GeV}$ o $0.3 \mathrm{GeV}<p_{\mathrm{T}}^{\text {iso }}<1 \mathrm{GeV}$.

La particular elección de $9 \mathrm{GeV}$ y $0.2 \mathrm{GeV}$ apunta a definir una región de solofondo, pero manteniendo suficiente estadística para calcular $R^{\prime}$.

Finalmente, si se toma en cuenta la contaminación de señal y las posibles correlaciones, la ecuación para el número esperado de jets falseando fotones resulta:

$$
N_{j \rightarrow \gamma}=N_{A}^{b}=\left[R^{\prime} \frac{N_{C}-N_{C}^{s}}{N_{D}-N_{D}^{s}}\left(1-\frac{N_{B}^{s}}{N_{B}}\right)\right] \times N_{B}=F F_{\text {ISO }} \times N_{B}
$$

Las incertezas sistemáticas se estiman del $100 \%$ en base a estudios de la variación de los $F F$ en variables no tenidas en cuenta en su determinación, como ser $E_{\mathrm{T}}^{\mathrm{miss}}$, $H_{\mathrm{T}}$ y variables angulares [46].

La Tabla 5.11 muestra los $F F$ resultantes $\left(F F_{\text {iso }}\right)$ y sus incertezas para los datos de 2015+2016, 2017 y 2018, para distintos bines de $\eta$ y $p_{\mathrm{T}}$. 


\begin{tabular}{c|l|cc}
\hline \hline Año & $p_{\mathrm{T}}[\mathrm{GeV}]$ & $|\eta|<1.37$ & $1.52<|\eta|<2.37$ \\
\hline \hline $2015+2016$ & {$[145-200]$} & $0.154 \pm 0.001$ & $0.171 \pm 0.001$ \\
& {$[200-250]$} & $0.123 \pm 0.001$ & $0.144 \pm 0.001$ \\
& {$[250-300]$} & $0.107 \pm 0.001$ & $0.139 \pm 0.001$ \\
& {$[300-350]$} & $0.100 \pm 0.001$ & $0.134 \pm 0.003$ \\
& {$[350-400]$} & $0.096 \pm 0.002$ & $0.140 \pm 0.004$ \\
& $>400$ & $0.091 \pm 0.002$ & $0.148 \pm 0.005$ \\
\hline 2017 & {$[145-200]$} & $0.162 \pm 0.001$ & $0.174 \pm 0.001$ \\
& {$[200-250]$} & $0.128 \pm 0.001$ & $0.147 \pm 0.001$ \\
& {$[250-300]$} & $0.115 \pm 0.001$ & $0.140 \pm 0.002$ \\
& {$[300-350]$} & $0.102 \pm 0.001$ & $0.141 \pm 0.003$ \\
& {$[350-400]$} & $0.098 \pm 0.002$ & $0.147 \pm 0.004$ \\
& $>400$ & $0.097 \pm 0.002$ & $0.157 \pm 0.005$ \\
\hline 2018 & {$[145-200]$} & $0.161 \pm 0.001$ & $0.174 \pm 0.001$ \\
& {$[200-250]$} & $0.128 \pm 0.001$ & $0.147 \pm 0.001$ \\
& {$[250-300]$} & $0.112 \pm 0.001$ & $0.139 \pm 0.001$ \\
& {$[300-350]$} & $0.104 \pm 0.001$ & $0.140 \pm 0.002$ \\
& {$[350-400]$} & $0.099 \pm 0.001$ & $0.147 \pm 0.004$ \\
& $>400$ & $0.094 \pm 0.001$ & $0.157 \pm 0.004$ \\
\hline \hline
\end{tabular}

Tabla 5.11: FF de fotones obtenidos con los datos de 2015+2016, 2017 y 2018 para diferentes valores de $p_{\mathrm{T}}$ tal y como se utilizan en el análisis, para las regiones barrel y endcap del detector. Las incertezas mostradas son estadísticas.

\subsubsection{Electrones falseando fotones}

En casos en los que un electrón de alto $p_{\mathrm{T}}$ es identificado erróneamente como un fotón, se espera una contaminación significativa de procesos del SM como $W / Z+$ jets y eventos $t \bar{t}$. Este fondo se estima pesando el número de eventos de electrones observados en una muestra de control de electrones (CSE) por la tasa de electrones considerados como fotones (Ec. 5.13). Estas muestras de control de electrones se obtienen invirtiendo los roles de los fotones y electrones en las diferentes regiones de control, señal y validación. Se requiere entonces un electrón aislado de alto $p_{\mathrm{T}}, \mathrm{y}$ los fotones de señal son vetados.

Para estimar la tasa de electrones falseando fotones, se utiliza un método basado en una muestra de eventos de datos $Z(\rightarrow e e)$ como se describe en [150, 147]. Dado que el bosón $Z$ no puede decaer directamente a un electrón y a un fotón, los eventos electrón-fotón que aparecen en el rango de masa del bosón $Z$ se corresponden a electrones mal identificados.

El fake factor de electrón a fotón se estima como:

$$
f_{e \gamma}=\frac{N_{e \gamma}}{N_{e e}}
$$

donde $N_{e \gamma}\left(N_{e e}\right)$ es el número de pares electrón-fotón (electrón-electrón) que se encuentran bajo el pico del $Z$ en la distribución de masa invariante, aquí definida en el rango $[86,96] \mathrm{GeV}$. Para evitar que los electrones provengan del decaimiento del $W$, solo se seleccionan los eventos con $E_{\mathrm{T}}^{\text {miss }}<40 \mathrm{GeV}$.

Para cada uno de los ee y e $e$, se realizan distintos ajustes en distintas regiones angulares del detector para estimar la contribución relativa de la señal y el fondo (de 
eventos no provenientes del decaimiento del bosón $Z$, en esta caso dominado por una combinación aleatoria de objetos). Las distintas regiones de estudio se organizan en base a que ambos objetos se reconstruyan en el barrel del calorímetro (BB), otra para que ambos objetos se encuentren en el endcap del calorímetro (EE), y otra para que un objeto se encuentre en el barrel y el otro en el endcap (BE). La Figura 5.19

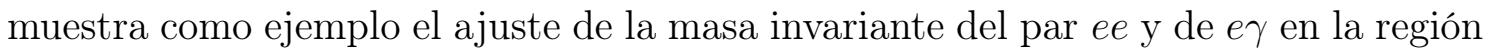
EE para los datos de 2017. Para estos ajustes, la señal correspondiente al $Z$ está modelada con una función double-sided Crystal-Ball. Finalmente, en base a estos ajustes se determina la contribución correspondiente a eventos de señal y fondo para cada caso de manera de poder determinar $N_{e e} \mathrm{y} N_{e \gamma}$ en cada región. Los pequeños fondos no resonantes que contaminan la producción de $Z(\rightarrow e e)$ son descriptos por un polinomio de segundo grado. Dependiendo de la categoría (BB, EE o BE) se aplica a cada evento un peso de $w=s /(s+b)$, donde $s(b)$ es la cantidad ajustada de señal (fondo) para la categoría y masa dadas, a medida que se cuentan eventos en bines de $p_{\mathrm{T}}-\eta$ para calcular los correspondientes fake factor como en la Ecuación 5.13.
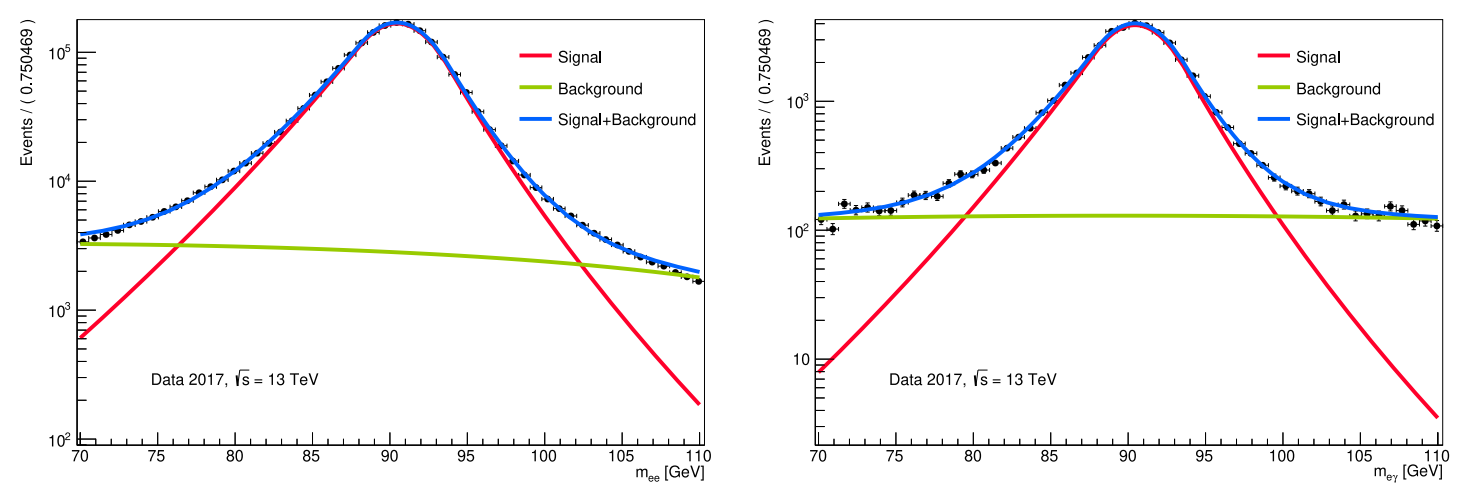

Figura 5.19: Ajuste de la masa invariante del par ee (izquierda) y de $e \gamma$ (derecha) en la región EE para datos de 2017.

La incerteza sistemática se estima variando el tamaño de la ventana de aceptación alrededor de la masa del bosón $Z(80-100 \mathrm{GeV})$ y no aplicando una sustracción de fondo a las muestras utilizadas para estimar el fondo (es decir, considerar los pesos anteriores como $w=1$ ). Esta última resulta ser la incerteza sistemática dominante.

Los electrones utilizados para estimar la contaminación de fondo en las diferentes regiones podrían ser, en principio, de tipo medium o tight, con aislamiento FCLoose. En ambos casos, la relación señal/fondo en la muestra $Z(\rightarrow e e)$ es alta, pero en el caso de la selección medium la cantidad de eventos es un poco mayor en las regiones de control y validación. Los fotones usados para este análisis son tight con aislamiento FixedCutTight.

Los valores de $f_{e \gamma}$ utilizados en este análisis en función de $|\eta|$ y $p_{\mathrm{T}}$ de los objetos se muestran en la Tabla 5.12 para los datos de $2015+2016,2017$ y 2018. Se puede ver que la tasa crece con $|\eta|$ correlacionado con el aumento del material del detector atravesado por los electrones y la correspondiente mayor tasa de reconstrucción de fotones como convertidos con una sola traza asociada. La Figura 5.20 muestra los resultados de la estimación de los factores $f_{\text {er }}$ para los datos de 2015+2016, 2017 y 2018.

Finalmente, como ya se ha mencionado, el número de eventos falsos esperados 


\begin{tabular}{|c|c|c|c|c|c|c|c|}
\hline \multirow{2}{*}{ Año } & \multirow{2}{*}{$|\eta|$} & \multirow{2}{*}{$p_{T}[\mathrm{GeV}]$} & \multirow{2}{*}{$f_{e \gamma}$} & \multirow{2}{*}{ Estadístico } & \multicolumn{2}{|c|}{ Sistemático } & \multirow{2}{*}{ Total } \\
\hline & & & & & $w=1$ & Rango & \\
\hline \multirow{12}{*}{$2015+2016$} & $0-0.6$ & $75-90$ & 0.0100 & $\overline{0.0003}$ & 0.0028 & $\overline{0.0006}$ & $\overline{0.0029}$ \\
\hline & $0-0.6$ & $90-145$ & 0.0126 & 0.0004 & 0.0036 & 0.0004 & 0.0036 \\
\hline & $0-0.6$ & $145-300$ & 0.0144 & 0.0009 & 0.0041 & 0.0006 & 0.0043 \\
\hline & $0.6-1.37$ & $75-90$ & 0.0141 & 0.0003 & 0.0037 & 0.0005 & 0.0037 \\
\hline & $0.6-1.37$ & $90-145$ & 0.0175 & 0.0005 & 0.0046 & 0.0003 & 0.0046 \\
\hline & $0.6-1.37$ & $145-300$ & 0.0195 & 0.0010 & 0.0051 & 0.0011 & 0.0053 \\
\hline & $1.52-1.82$ & $75-90$ & 0.0298 & 0.0010 & 0.0039 & 0.0001 & 0.0040 \\
\hline & $1.52-1.82$ & $90-145$ & 0.0361 & 0.0014 & 0.0048 & 0.0011 & 0.0051 \\
\hline & $1.52-1.82$ & $145-300$ & 0.0455 & 0.0033 & 0.0059 & 0.0016 & 0.0069 \\
\hline & $1.82-2.37$ & $75-90$ & 0.0526 & 0.0012 & 0.0060 & 0.0026 & 0.0067 \\
\hline & $1.82-2.37$ & $90-145$ & 0.0689 & 0.0018 & 0.0080 & 0.0014 & 0.0083 \\
\hline & $1.82-2.37$ & $145-300$ & 0.0742 & 0.0043 & 0.0082 & 0.0016 & 0.0093 \\
\hline \multirow{12}{*}{2017} & $0-0.6$ & $75-90$ & 0.0120 & 0.0003 & 0.0028 & 0.0003 & 0.0028 \\
\hline & $0-0.6$ & $90-145$ & 0.0155 & 0.0004 & 0.0036 & 0.0004 & 0.0037 \\
\hline & $0-0.6$ & $145-300$ & 0.0166 & 0.0009 & 0.0039 & 0.0004 & 0.0040 \\
\hline & $0.6-1.37$ & $75-90$ & 0.0144 & 0.0003 & 0.0031 & 0.0007 & 0.0032 \\
\hline & $0.6-1.37$ & $90-145$ & 0.0172 & 0.0004 & 0.0037 & 0.0009 & 0.0039 \\
\hline & $0.6-1.37$ & $145-300$ & 0.0198 & 0.0010 & 0.0043 & 0.0011 & 0.0046 \\
\hline & $1.52-1.82$ & $75-90$ & 0.0313 & 0.0010 & 0.0039 & 0.0003 & 0.0040 \\
\hline & $1.52-1.82$ & $90-145$ & 0.0368 & 0.0013 & 0.0047 & 0.0008 & 0.0049 \\
\hline & $1.52-1.82$ & $145-300$ & 0.0458 & 0.0032 & 0.0058 & 0.0007 & 0.0066 \\
\hline & $1.82-2.37$ & $75-90$ & 0.0545 & 0.0012 & 0.0060 & 0.0006 & 0.0062 \\
\hline & $1.82-2.37$ & $90-145$ & 0.0660 & 0.0016 & 0.0074 & 0.0008 & 0.0076 \\
\hline & $1.82-2.37$ & $145-300$ & 0.0705 & 0.0039 & 0.0075 & 0.0003 & 0.0085 \\
\hline \multirow{12}{*}{2018} & $0-0.6$ & $75-90$ & 0.0117 & 0.0003 & 0.0028 & 0.0005 & 0.0028 \\
\hline & $0-0.6$ & $90-145$ & 0.0146 & 0.0004 & 0.0035 & 0.0004 & 0.0036 \\
\hline & $0-0.6$ & $145-300$ & 0.0150 & 0.0008 & 0.0037 & 0.0003 & 0.0038 \\
\hline & $0.6-1.37$ & $75-90$ & 0.0143 & 0.0003 & 0.0030 & 0.0008 & 0.0031 \\
\hline & $0.6-1.37$ & $90-145$ & 0.0178 & 0.0004 & 0.0037 & 0.0006 & 0.0037 \\
\hline & $0.6-1.37$ & $145-300$ & 0.0197 & 0.0009 & 0.0042 & 0.0003 & 0.0043 \\
\hline & $1.52-1.82$ & $75-90$ & 0.0312 & 0.0009 & 0.0025 & 0.0010 & 0.0028 \\
\hline & $1.52-1.82$ & $90-145$ & 0.0387 & 0.0012 & 0.0031 & 0.0003 & 0.0033 \\
\hline & $1.52-1.82$ & $145-300$ & 0.0410 & 0.0027 & 0.0032 & 0.0000 & 0.0042 \\
\hline & $1.82-2.37$ & $75-90$ & 0.0534 & 0.0011 & 0.0038 & 0.0010 & 0.0041 \\
\hline & $1.82-2.37$ & $90-145$ & 0.0700 & 0.0015 & 0.0051 & 0.0000 & 0.0053 \\
\hline & $1.82-2.37$ & $145-300$ & 0.0802 & 0.0037 & 0.0056 & 0.0015 & 0.0069 \\
\hline
\end{tabular}

Tabla 5.12: Fake factor para electrones falseando fotones para diferentes regiones de $\eta$ y $p_{\mathrm{T}}$ como se usan en el análisis nominal, para el conjunto de datos de $2015+2016$, 2017 y 2018. Las incertezas estadísticas y sistemáticas se muestran por separado. 


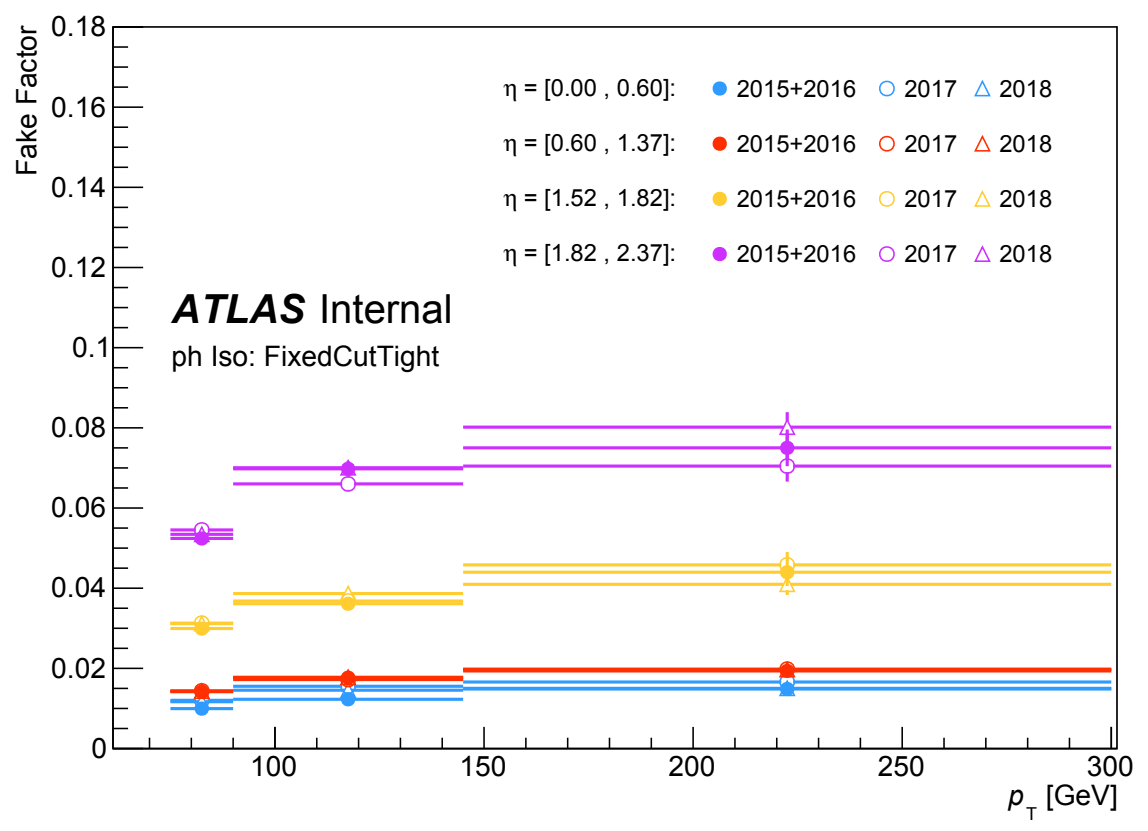

Figura 5.20: Fake factor para electrones falseando fotones como función de $p_{\mathrm{T}}$ para diferentes valores de $|\eta|$, para el conjunto de datos de 2015+2016, 2017 y 2018.

en cualquier región (R) se obtiene pesando, con los correspondientes fake factors, el número de eventos observados en la muestra de control de electrones (CSE) donde el rol del fotón es reemplazado por un electrón aislado:

$$
N_{e \rightarrow \gamma}^{R}\left(\eta, p_{T}\right)=f_{e \gamma}\left(\eta, p_{T}\right) \cdot N_{\mathrm{CSE}}^{R}\left(\eta, p_{T}\right)
$$

Para este fondo se consigue diseñar una región de validación dedicada (VRE) con la selección de eventos descripta en la Tabla 5.13, para validar la precisión de las predicciones de fondo correspondientes basadas en los fake factors calculados. El conjunto de cortes selecciona predominantemente eventos $\mathrm{W}(e \nu)+$ jets, donde un boosted $W$ decae en neutrinos colineales (con un alto $E_{\mathrm{T}}^{\text {miss }}$ ) y un electrón de alto $p_{\mathrm{T}}$ (erróneamente identificado como fotón). La validación de las estimaciones en esta región se muestra en conjunto con el resto de las regiones de validación en el siguiente capítulo.

\subsection{Tratamiento estadístico e interpretación de los resultados}

En la Sección 5.3 se describió el procedimiento general para la correcta estimación en las regiones de señal de los fondos principales que se evalúan de muestras MC, a partir de la construcción de regiones de control y validación. Se inicia esta sección extendiendo dicha discusión para explicitar la forma en la que se obtiene esta estimación de los fondos.

De manera general, para un dado fondo $i$ perteneciente al conjunto de fondos principales $\mathrm{BKG}$, se define una $\mathrm{CR}_{i}$. El número de eventos del dado fondo $i$ en datos en esta región es: 


\begin{tabular}{|l|r|}
\hline & VRE \\
\hline$N_{\text {fotones }}$ & $\geq 1$ \\
$p_{\mathrm{T}}^{\text {leading- }}$ & $>145 \mathrm{GeV}$ \\
$N_{\text {leptones }}$ & - \\
$N_{\text {jets }}$ & $\geq 1$ \\
$N_{b-\text { jets }}$ & $\geq 1$ \\
$\Delta \phi\left(\right.$ jet, $\left.E_{\mathrm{T}}^{\text {miss }}\right)$ & $>0.4$ \\
$\Delta \phi\left(\gamma, E_{\mathrm{T}}^{\text {miss }}\right)$ & $<0.4$ \\
$E_{\mathrm{T}}^{\text {miss }}$ & $>200 \mathrm{GeV}$ \\
$H_{\mathrm{T}}$ & {$[100,1600] \mathrm{GeV}$} \\
\hline
\end{tabular}

Tabla 5.13: Selección de eventos para la región de validación VRE, diseñada para validar el fondo $e \rightarrow \gamma$.

$$
N_{i, \text { datos }}^{\mathrm{CR}_{i}}=N_{\text {datos }}^{\mathrm{CR}_{i}}-\sum_{j \neq i \in \mathrm{BKG}} \frac{N_{j, \text { datos }}^{\mathrm{CR}_{j}}}{N_{j, M C}^{\mathrm{CR}_{j}}} N_{j, M C}^{\mathrm{CR}_{i}}-\sum_{k \in \mathrm{bkg}} N_{k, M C}^{\mathrm{CR}_{i}}
$$

donde $N_{\text {datos }}^{\mathrm{CR}_{i}}$ es el número de eventos total observados en datos, $N_{i, M C}^{\mathrm{CR}_{i}}$ es el número de eventos en MC para el fondo $i, \mathrm{y}$ bkg es el conjunto de los fondos menores evaluados solo con MC (aquellos que no tienen una CR asociada). Se definen a partir de lo anterior, los factores de normalización para cada fondo $i$ como:

$$
\mu_{i}^{s f}=\frac{N_{i, \text { datos }}^{\mathrm{CR}_{i}}}{N_{i, M C}^{\mathrm{CR}}}
$$

que se determinan en un ajuste simultáneo en todas las regiones de control. Este resultado se usa en la estimación de eventos de fondo en cada SR, a través de los ya mencionados factores de transferencia, que se definen como el cociente:

$$
t_{i}=\frac{N_{i, M C}^{\mathrm{SR}}}{N_{i, M C}^{\mathrm{CR}_{i}}}
$$

y el número de eventos de fondo total esperado en la SR (se supone aquí que no hay señal) queda entonces como:

$$
N_{\mathrm{SR}}=\sum_{i \in \mathrm{BKG}} \mu_{i}^{s f} t_{i} N_{i, M C}^{\mathrm{CR}_{i}}+\sum_{k \in \mathrm{bkg}} N_{k, M C}^{\mathrm{SR}}
$$

\subsubsection{Test de hipótesis}

El objetivo de una búsqueda como la presentada en esta tesis, es poder especificar cuán bueno es el acuerdo entre los datos observados y una dada hipótesis, como ser las predicciones del número de eventos para una SR. Los test de hipótesis buscan discriminar entre una hipótesis nula $\left(H_{0}\right)$ y una hipótesis alternativa $\left(H_{1}\right)$. Por ejemplo, si se quiere calcular una significancia de descubrimiento, $H_{0}$ es la hipótesis 
de solo-fondo, mientras que $H_{1}$ supone la presencia de señal además del fondo. Para distinguir entre estas hipótesis se define un parámetro $\mu$, llamado intensidad de señal, que es cero para la hipótesis de solo-fondo y uno para la hipótesis de señal nominal. Los estadísticos de prueba se construyen para estudiar la compatibilidad de los datos observados con una hipótesis y son función de estos datos. Para cada hipótesis el estadístico de prueba tiene una función de distribución de probabilidad $f\left(q_{\mu} \mid H\right)$.

Un estadístico de prueba condensa todo el poder de discriminación S-B en un número. Los discriminadores más potentes son los likelihoods ratios, de acuerdo al lema de Neyman-Pearson [151] y se definen como:

$$
q_{\mu}=-2 \ln \left[\frac{L(\text { data } \mid \mu)}{L(\text { data } \mid \hat{\mu})}\right]
$$

según se detalla en la siguiente sección.

El nivel de acuerdo entre los datos y una dada hipótesis $\mathrm{H}$, es decir, la probabilidad de que un estadístico de prueba sea tan grande como su valor observado, suponiendo que $\mathrm{H}$ es correcta, viene dada por el valor- $p$ que se define como:

$$
\text { valor }-p=P\left(q>q_{o b s} \mid H\right)=\int_{q_{o b s}}^{\infty} f(q \mid H) d q
$$

donde f es la función de densidad de probabilidad y $q_{o b s}$ es el valor del estadístico de prueba observado en datos. Un valor-p chico significa que la hipótesis no coincide con los datos observados y $\mathrm{H}$ se considera excluida cuando el valor-p es menor que un determinado valor $\alpha$. Se suele convertir el valor-p $(p)$ en una significancia Z, como el número de desviaciones estándar a partir de las cuales una variable aleatoria distribuida normalmente tiene un área igual al valor-p. Se tiene entonces:

$$
Z=\Phi^{-1}(1-p)
$$

donde $\Phi^{-1}$ es el cuantil de la distribución normal.

Si se quiere evaluar la hipótesis de sólo fondo $\left(H_{b}\right)$, se tiene el valor $p_{b}$ que resulta:

$$
p_{b}=P\left(q \leq q_{o b s} \mid H_{b}\right)=1-\int_{q_{o b s}}^{\infty} f\left(q \mid H_{b}\right) d q
$$

a partir del cual se define el nivel de confianza de la hipótesis de solo-fondo $\mathrm{CL}_{b}=$ $1-p_{b}$. Por convención, si la probabilidad $p_{b}$ de observar unos ciertos datos bajo la suposición de $H_{b}$ verdadera, es menor a $2.87 \cdot 10^{-7}$, es decir $Z_{0}>5$, se rechaza la hipótesis de solo-fondo.

Si lo que se busca es evaluar la hipótesis de señal $\left(H_{s}\right)$, se tiene el valor-p, $p_{\mu}$ :

$$
p_{\mu}=P\left(q \leq q_{o b s} \mid H_{s}\right)=\int_{q_{o b s}}^{\infty} f\left(q \mid H_{s}\right) d q
$$

donde para excluir a la hipótesis de señal se pide $p_{\mu}<0.05$. Lo que se conoce como el límite superior al $95 \%$ de nivel de confianza (CL) en el parámetro de interés. 
Sin embargo, esta forma de proceder puede tener problemas si los datos observados tienen una fluctuación (hacia abajo) con respecto al fondo esperado, ya que se podría excluir incorrectamente la hipótesis de señal. Para combatir la anterior inferencia en la señal, se usa la convención del LHC que, en vez de requerir $C L_{s+b}=5 \%$, pide:

$$
C L s=\frac{p_{\mu}}{1-p_{b}}=\frac{C L_{s+b}}{C L_{b}}=\frac{\int_{q_{\mu}^{o b s}}^{\infty} f\left(q_{\mu} \mid H_{s}\right) d q_{\mu}}{\int_{0}^{q_{\mu}^{o b s}} f\left(q_{\mu} \mid H_{b}\right) d q_{\mu}}
$$

Por lo tanto, el límite superior $\mu_{u p}$ se obtiene para $C L s=0.05$, y se rechazan los valores de $\mu$ si $\mu<\mu_{u p}$ con un nivel de confianza del $95 \%$.

\subsubsection{Construcción del modelo}

La función likelihood se construye como el producto de las distribuciones de Poisson para el número de eventos en cada región y de un término gaussiano que tiene en cuenta las incertezas sistemáticas

$$
\begin{aligned}
L\left(\boldsymbol{n}, \boldsymbol{\theta}^{\mathbf{0}} \mid \mu_{s i g}, \boldsymbol{\mu}_{s \boldsymbol{f}}, \boldsymbol{b}, \boldsymbol{\theta}\right) & =P_{S R} \cdot P_{C R} \cdot C_{s y s} \\
& =\operatorname{Pois}\left(n_{S R} \mid \lambda_{S R}\left(\mu_{s i g}, \boldsymbol{b}, \boldsymbol{\theta}\right)\right) \cdot \prod_{i \in C R} \operatorname{Pois}\left(n_{i} \mid \lambda_{i}\left(\mu_{s i g}, \mu_{s f}, \boldsymbol{b}, \boldsymbol{\theta}\right)\right) \cdot C_{s y s}\left(\boldsymbol{\theta}^{\mathbf{0}}, \boldsymbol{\theta}\right)
\end{aligned}
$$

donde i recorre cada una de las CR, los parámetros $\lambda$ son los valores esperados de las distribuciones de Poisson que dependen de las predicciones $b$ de los fondos, de los factores de normalización $\left(\mu_{s f}\right)$, de los parámetros nuissance $(\boldsymbol{\theta})$ que modelan las incertezas sistemáticas con valor central $\boldsymbol{\theta}^{\mathbf{0}}$ (alrededor de los cuales los $\boldsymbol{\theta}$ pueden variarse para maximizar el likelihood) y el parámetro $\mu_{\text {sig }}$ que es la intensidad de señal. Cuando la intensidad de señal se fija en cero, se tiene la función likelihood para la hipótesis de solo-fondo. La función de distribución de probabilidad gaussina $C_{\text {sys }}\left(\boldsymbol{\theta}^{\mathbf{0}}, \boldsymbol{\theta}\right)$ incluye en el modelo las incertezas sistemáticas. Para cada parámetro nuissance independiente, $C_{\text {sys }}$ es el producto de las distribuciones correspondientes a mediciones auxiliares que describen las incertezas sistemáticas.

\section{Distribución del estadístico de prueba}

La hipótesis de sólo fondo da $\mu=0$. La hipótesis de señal + fondo tiene $\mu=1$ (aunque no está acotada y se puede hacer más grande si, por ejemplo, se quiere 2 veces la señal, etc). En particular, se tiene que:

$$
q_{1}=-2 \ln \left[\frac{L(\text { data } \mid \mu=1)}{L(\text { data } \mid \hat{\mu})}\right]
$$

donde el numerador es el likelihood suponiendo la intensidad de señal nominal, y el denominador es el likelihood del mejor ajuste, donde $\hat{\mu}$ es el mejor ajuste del valor de $\mu$.

El valor de $q_{1}$ en datos es ahora la medición. La distribución de $q_{1}$ no es calculable, pero puede obtenerse de pseudo-experimentos (toys). Se deben generar un gran 
número de pseudo-experimentos con un dado valor de $\mu$, calcular $q_{1}$ para cada uno y así obtener su distribución.

De $q_{o b s}$ y estas distribuciones del estadístico de prueba, se pueden poner límites o calcular significancias de descubrimiento de manera similar a lo que se mostró antes con el experimento de conteo y las distribuciones de Poisson.

\section{Incertezas sistemáticas}

$\mathrm{Al}$ incluir las incertezas sistemáticas, se tiene que el likelihood es $L($ data $\mid \vec{\mu}, \vec{\theta})=$ $\operatorname{Pois}\left(N_{i} \mid \mu \cdot s_{i}(\theta)+b_{i}(\theta)\right) \times P(\tilde{\theta}, \theta)$

$\mathrm{Y}$ el estadístico de prueba es el Profile Likelihood Ratio, que se escribe como:

$$
\tilde{q}_{\mu}=-2 \ln \left[\frac{L(\text { data } \mid \mu, \hat{\hat{\theta}})}{L(\operatorname{data} \mid \hat{\mu}, \hat{\theta})}\right]
$$

con $0 \leq \hat{\mu} \leq \mu$, y el numerador es el likelihood del mejor ajuste para un dado valor fijo de $\mu$, mientras que el denominador es el likelihood del mejor ajuste.

\subsubsection{Flujo de una búsqueda}

El flujo típico de una búsqueda como la realizada en la presente tesis puede resumirse de forma esquemática en los siguientes pasos:

- En primer lugar se normalizan los fondos dominantes a los datos en un ajuste simultáneo de las regiones de control.

- Se extrapolan los fondos a las regiones de validación y señal, usando los factores de transferencia (definidos como el cociente de eventos MC en la CR y la VR o la SR).

- Si hay buen acuerdo en las VR, se confía en la predicción de fondo en las SRs $\mathrm{y}$ se procede a incluir los datos de dichas regiones (unblind).

- Si no hay exceso de datos sobre los fondos del SM, se agrega la predicción de señal, se interpreta y se determinan los límites en los parámetros correspondientes del modelo.

- Si hay exceso, se evalúa la significancia, que puede ser evidencia $(>3 \sigma)$ o descubrimiento $(>5 \sigma)$.

Los ajustes que se realizan son entonces:

Ajuste de solo-fondo:

Se estima el fondo en las regiones de validación y de señal, incluyendo solo las CR en el ajuste a los datos. No hay señal en el ajuste, es decir que el likelihood se supone con una intensidad de señal $\mu_{\text {sig }}=0$. Las regiones de control se consideran libres de contaminación de señal. A su vez, en la expresión 5.25 no hay término de la SR. A partir de este ajuste y la extrapolación de fondo a las regiones de señal, se puede ver si existe un exceso de datos con respecto al fondo en las SR.

Ajuste de señal dependiente del modelo o Ajuste de exclusión:

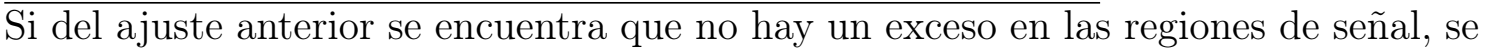


determinan límites de exclusión en un modelo específico. En este caso, en el ajuste simultáneo se tienen en cuenta tanto los fondos como la señal en las CR y en la SR. De esta forma se incluye la contaminación de señal en las regiones de control. El likelihood se supone con una intensidad de señal $\mu_{\text {sig }} \neq 0$. Se hace un ajuste de forma independiente para cada región de señal y se repite para cada punto de señal para los distintos parámetros que la describen.

Ajuste de señal independiente del modelo o Ajuste de descubrimiento:

Se obtienen límites superiores de manera independiente del modelo, en el número de eventos BSM, más allá de la predicción del fondo. En el ajuste se tienen en cuenta el fondo y una señal dummy en las CR y en las SR. El likelihood se supone con solo fondo. 


\section{Capítulo 6}

\section{Resultados e interpretación de la búsqueda de SUSY en estados finales con fotones $+E_{\mathrm{T}}^{\text {miss }}+$ jets}

En este capítulo se presentan los resultados obtenidos en el contexto de la búsqueda de nueva física, producto de la estrategia planteada en el capítulo anterior. En primer lugar se hace una descripción de las incertezas sistemáticas que afectan el análisis, tanto experimentales como teóricas. Luego se muestran los resultados en las distintas regiones de control, validación y señal, que surgen de realizar un ajuste de solo-fondo utilizando los datos de todo el Run-2. Se muestra el buen acuerdo general obtenido entre la predicción del fondo del SM y los datos en las CR y VR, y las estimaciones del fondo en las regiones de señal. A continuación se presentan los resultados en las regiones de señal para los datos de 2015 y 2016, y a partir del número de eventos observados en dichas regiones, se computan los límites dependientes e independientes del modelo de SUSY que se estudia.

\subsection{Incertezas sistemáticas}

Las incertezas sistemáticas afectan a todos los procesos de fondo estimados, ya sea utilizando MC o un método basado en datos, como también a las predicciones de señal. Estas incertezas sistemáticas proceden principalmente de dos tipos de fuentes: las experimentales y las teóricas, y pueden afectar al número esperado de eventos tanto en las regiones de control y validación, como en las de señal.

\subsubsection{Incertezas experimentales}

A continuación se describen los procedimientos para evaluar las incertezas comunes a todos los procesos y el número de parámetros nuisance (NP) a ser considerados. Entre ellas se incluyen las incertezas en la escala y resolución de energía de los distintos objetos físicos, las eficiencias de reconstrucción e identificación, y la luminosidad integrada registrada por ATLAS. El caso especial de las incertezas sistemáticas que afectan a las estimaciones data-driven para electrones y jets considerados como fotones, se discute en las Secciones 5.4.2 y 5.4.1, respectivamente, y están resumidas en la Sección 6.1.2. 
En las regiones de señal, las incertezas dominantes provienen de la escala y resolución de la energía de los jets.

\section{Incerteza en la luminosidad}

La incerteza en la luminosidad integrada combinada de Run-2 es del 1.7\% [72]. Se deriva siguiendo una metodología similar a la que se detalla en [152], de una calibración preliminar de la escala de luminosidad usando barridos de separación en $x-y$ del haz, conocidos como scans de Van der Meer.

\section{Incertezas de la identificación, escala de energía y resolución de fotones}

Las incertezas en la identificación de fotones (PH_EFF_ID_Uncertainty) son estimadas en medidas combinadas data-driven utilizando, entre otras, técnicas de identificación y pruebas en eventos radiativos del bosón Z [153, 154]. Para determinar las incertezas en las corrección de escala de energía (EG_SCALE_ALL) y la resolución (EG_RESOLUTION_ALL), se aplicaron respectivamente dichas correcciones con valores superiores e inferiores a las nominales en una desviación estándar [155, 156]. Estas desviaciones se aplicaron independientemente a cada muestra de MC y, a partir de las diferencias en el número de eventos seleccionados en cada caso, se asignaron las incertezas sistemáticas [157].

Los sistemáticos asociados a la determinación del aislamiento de los objetos se realiza en base a estudios MC a los que se les agregan o quitan correcciones basadas en estudios con datos [158].

\section{Incertezas de la identificación, escala de energía y resolución de leptones}

Para los electrones [159] y los muones [86], de manera similar a los fotones, la incerteza referida a la eficiencia de la identificación, la escala de energía y la resolución se determinan a partir de muestras de control $Z \rightarrow l^{+} l^{-}, J / \psi \rightarrow l^{+} l^{-}$ y $W^{ \pm} \rightarrow l^{ \pm} \nu$ ([160], [155] y [161]). Los parámetros nuisance a ser considerados son, respecto a los electrones, escala de energía (EG_SCALE_ALL), eficiencias de identificación, reconstrucción y aislamiento de electrones (EL_EFF_ID, EL_EFF_Reco y EL_EFF_Iso). En relación a los muones se tiene, la resolución y escala del momento (MUON_ID, MS y MUON_SCALE), y las eficiencias de reconstrucción y aislamiento (MUON_EFF y MUON_ISO).

\section{Incertezas asociadas a los jets}

Para los jets, las incertezas en la escala de energía y la resolución se derivan siguiendo el procedimiento descripto en [102] y [162]. Se consideran las siguientes incertezas:

- Incerteza en la resolución en energía de los jets (JER_EffNP_\{1, . ,7\}): variaciones de $\pm 1 \sigma$ en el smearing de los jets (smear jets de MC a datos, para corregir la resolución en MC) calculada usando eventos de di-jets y data zero bias usando conos aleatorios. Se tienen 8 NP.

- Incerteza en la escala de energía de los jets (JET_\{[...]\}): variaciones de $\pm 1 \sigma$ en la escala del momento del jet. Las incertezas de la escala provienen de 
la intercalibración en $\eta$ de di-jets, balance de $\mathrm{Z}+$ jet, balance de gamma + jet, balance de multi-jet, propagación de incertezas de partículas individuales y del test-beam, incertezas de pile-upe incertezas relacionadas con el sabor, que posteriormente se combinan en un conjunto de $29 \mathrm{NP}$.

- Incerteza en la eficiencia del etiquetado de sabores Flavor-tagging (FT_EFF_\{B,C,Light\},FT_EFF_extrapolation $\{$ charm $\}$ ): variaciones de $\pm 1 \sigma$ en la incerteza de los factores de escala que corrigen la tasa de etiquetado en simulaciones, para coincidir con aquella en datos.

- Incerteza en JVT (JET_JVTEff): variaciones de $\pm 1 \sigma$ que tienen en cuenta la contaminación residual proveniente de jets de pile-up después de aplicar supresión de pile-up y la elección del generador de MC.

\section{Incertezas para el término soft de $E_{\mathrm{T}}^{\text {miss }}$}

Para $E_{\mathrm{T}}^{\text {miss }}$, las incertezas de todos los objetos subyacentes a partir de los cuales se construye, se propagan, y se incluyen incertezas adicionales por la contribución del término soft. Los sistemáticos del término soft (TST) de $E_{\mathrm{T}}^{\text {miss }}$ se derivan de la comparación de la escala y resolución de energía de los datos 2015 con MC, a partir de comparaciones entre generadores. El impacto de las incertezas de la escala se estima variando esas escalas hacia arriba y hacia abajo usando el paquete de software METUtilities-00-02-46 [163]. Esta herramienta provee las incertezas (MET_SoftTrk_ResoPara, MET_SoftTrk_ResoPerp, MET_SoftTrk_ScaleUp) para el término soft de $E_{\mathrm{T}}^{\mathrm{miss}}$.

\section{Pile-up}

Como se menciona en la Sección 5.2, el factor de peso nominal se determina para hacer coincidir las interacciones medias por cruce del MC con las de los datos. La incerteza sobre el repesado se evalúa cambiando el número medio de interacciones por cruce de paquetes de protones en $\pm 10 \%$.

\subsubsection{Incertezas originadas en métodos data-driven}

Como se discute en la Sección 5.4, los fondos con fotones falsos (jet $\rightarrow \gamma$ fakes y $e \rightarrow \gamma$ fakes) se estiman utilizando métodos data-driven. Los métodos implican los siguientes pasos: i) el cálculo de los fake factors utilizando datos; ii) el pesado de los eventos en una muestra de control con estos fake factors para la estimación final del fondo.

Hay dos tipos diferentes de incertezas que afectan esta estrategia. La incerteza sistemática a partir del método utilizado para estimar los fake factors y la incerteza estadística de la muestra de control. La incerteza sistemática que afecta a los fake factors se discute en las Secciones 5.4.1 y 5.4.2. Estas incertezas se incorporan al ajuste simultáneo como un parámetro nuisance habitual para cada uno de los fondos. Las incertezas estadísticas de las muestras de control se incorporan en el ajuste como parámetros nuisance. 


\subsubsection{Incertezas teóricas}

Estas incertezas afectan las predicciones basadas en MC de los fondos tanto en las regiones de señal como de control y validación. Éstas son estimadas a partir de la comparación entre eventos simulados con distintos generadores MC y de variaciones en los parámetros de entrada utilizados para la generación de eventos. La incerteza teórica total para el modelo de señal se discute en la Sección 5.2.1.

\section{Producción de fotones directos, $t \bar{t} \gamma, W \gamma \mathbf{y} Z \gamma$}

La incerteza teórica para la producción de $\gamma+$ jets, $t \bar{t} \gamma, W \gamma$ y $Z \gamma$ asociada a la elección de la simulación MC se evalúa comparando los valores nominales de la muestra de SHERPA con las variaciones generadas cambiando las escalas de renormalización, factorización, resumación y las escalas de combinación CKKW a nivel generador [164, 134]. Además, se tienen en cuenta las variaciones en la generación de los eventos con respecto a los parámetros por defecto, considerando diferentes familias de PDFs e incertezas [165].

Dependiendo de los pesos disponibles en cada muestra, una variación de las PDF se lleva a cabo en primer lugar, siguiendo las recomendaciones de [166, 167, 35, 168], utilizando LHAPDF con el conjunto de PDF CT14, NNPDF (NNPDF30_nnlo_as_0118) y MMHT (MMHT2014nnlo68cl) para realizar el re-pesado de las PDF y determinar el valor de la incerteza como:

$$
\Delta=\frac{1}{2}\left[\operatorname{máx}\left(\Delta_{\mathrm{CT} 14}^{+}, \Delta_{\mathrm{NNPDF}}^{+}, \Delta_{\mathrm{MMHT}}^{+}\right)-\operatorname{mín}\left(\Delta_{\mathrm{CT} 14}^{-}, \Delta_{\mathrm{NNPDF}}^{-}, \Delta_{\mathrm{MMHT}}^{-}\right)\right]
$$

Además, se utilizaron variaciones en las escalas de renormalización $\left(\mu_{R}\right)$ y factorización $\left(\mu_{F}\right)$. En particular se utilizaron siete pares de variaciones de $\mu_{R} \mathrm{y} \mu_{F}$ con: $\left\{\mu_{R}, \mu_{F}\right\} \times\{0.5,0.5\},\{1,0.5\},\{0.5,1\},\{1,1\},\{2,1\},\{1,2\}$ y $\{2,2\}$.

\subsection{Ajuste de solo-fondo para todo Run-2}

Como se mencionó en la Sección 5.5, para estimar el fondo en las regiones de validación y de señal se realiza, considerando solo las regiones de control, un ajuste simultáneo a los datos, incluyendo todas las muestras de fondo y sin incluir la señal. De esta forma, las predicciones obtenidas son independientes de las VR y SR, y el número de eventos esperado en estas regiones puede ser comparado sin sesgo con los valores observados. A continuación se presentan los resultados en base a este ajuste, en cada una de las regiones de control, validación y señal.

\subsubsection{Resultados en las regiones de control}

Cada una de las tres regiones de control, descriptas en la Sección 5.3.2, se definió para estar enriquecida en un fondo particular, estar cinemáticamente cerca de las regiones de señal pero, al mismo tiempo, ser ortogonales a éstas de manera de reducir la contaminación de señal (como se mostró en la Figura 5.16).

En la Tabla 6.1 se muestra una comparación de las predicciones de fondo (discriminando cada una de las componentes) y los datos observados en las CR, antes y después del ajuste, para el conjunto de datos completo de Run-2. En la parte 


\begin{tabular}{|c|c|c|c|}
\hline Regiones de Control & CRQ & CRW & CRT \\
\hline Eventos Observados & 1719 & 2273 & 1293 \\
\hline Eventos del SM Esperados & $1719.35 \pm 58.92$ & $2273.10 \pm 64.92$ & $1293.11 \pm 42.12$ \\
\hline$e \rightarrow \gamma$ fakes & $16.07 \pm 3.25$ & $82.64 \pm 15.79$ & $184.88 \pm 38.09$ \\
\hline$j \rightarrow \gamma$ fakes & $73.19 \pm 72.94$ & $150.31 \pm 149.75$ & $76.17 \pm 75.92$ \\
\hline$\gamma+$ jets & $1491.17 \pm 100.79$ & $16.41 \pm 12.75$ & $1.56_{-1.56}^{+2.83}$ \\
\hline$W \gamma$ & $29.35 \pm 5.07$ & $1799.19 \pm 184.10$ & $44.64 \pm 8.06$ \\
\hline$Z(\rightarrow \ell \ell) \gamma$ & $3.03 \pm 1.13$ & $50.04 \pm 6.79$ & $4.42 \pm 1.48$ \\
\hline$Z(\rightarrow \nu \nu) \gamma$ & $11.54 \pm 3.23$ & $0.14 \pm 0.06$ & $0.00 \pm 0.00$ \\
\hline$t \bar{t} \gamma$ & $44.84 \pm 7.04$ & $173.27 \pm 24.62$ & $980.72 \pm 107.57$ \\
\hline$\gamma \gamma / W \gamma \gamma / Z \gamma \gamma$ & $50.17 \pm 15.36$ & $1.09 \pm 0.76$ & $0.71 \pm 0.71$ \\
\hline Eventos SM antes del ajuste & 3088.70 & 2399.67 & 1051.31 \\
\hline Antes del ajuste $e \rightarrow \gamma$ fakes & 16.06 & 82.57 & 184.68 \\
\hline Antes del ajuste $j \rightarrow \gamma$ fakes & 73.08 & 150.09 & 76.05 \\
\hline Antes del ajuste $\gamma+$ jets & 2869.22 & 31.56 & 3.04 \\
\hline Antes del ajuste $W \gamma$ & 31.92 & 1954.54 & 48.53 \\
\hline Antes del ajuste $Z(\rightarrow \ell \ell) \gamma$ & 3.03 & 50.04 & 4.43 \\
\hline Antes del ajuste $Z(\rightarrow \nu \nu) \gamma$ & 11.54 & 0.14 & 0.00 \\
\hline Antes del ajuste $t \bar{t} \gamma$ & 33.58 & 129.65 & 733.87 \\
\hline Antes del ajuste $\gamma \gamma / W \gamma \gamma / Z \gamma \gamma$ & 50.28 & 1.09 & 0.71 \\
\hline Pureza del fondo & $93 \%$ & $81 \%$ & $70 \%$ \\
\hline Factor de normalización $(\mu)$ & $0.52 \pm 0.24$ & $0.92 \pm 0.12$ & $1.34 \pm 0.16$ \\
\hline
\end{tabular}

Tabla 6.1: Resultados del ajuste de solo-fondo en las regiones de control para los datos de todo Run-2, correspondientes a $139 \mathrm{fb}^{-1}$. Los valores esperados nominales (valores antes del ajuste) se muestran para su comparación. Las incertezas consideradas son estadísticas y sistemáticas.

inferior de la tabla se muestran también los valores obtenidos para los factores de normalización $(\mu)$ y el grado de pureza del fondo correspondiente en cada CR.

Las distribuciones de algunas de las variables representativas, utilizadas para definir las regiones de señal, se muestran en las Figuras 6.1, 6.2 y 6.3 para CRQ, CRW y CRT, respectivamente. Dichas distribuciones son el resultado de aplicar los factores de normalización obtenidos en el ajuste simultáneo en las regiones de control. Se agregan también los datos con el fin de evaluar el modelado del fondo. Todas las figuras muestran un buen acuerdo entre los datos observados y las predicciones de fondo.

\subsubsection{Resultados en las regiones de validación}

Los resultados obtenidos para los factores de normalización se utilizan para estimar el fondo en las regiones de validación, en las cuales se estudian las distintas distribuciones para verificar el correcto modelado de los fondos, antes de la extrapolación a las regiones de señal. Las distribuciones y tablas se muestran con los valores resultantes de la aplicación de los factores de normalización que se obtuvieron del ajuste simultáneo a las regiones de control.

El número de eventos esperados (después del ajuste) y observados en las regiones de validación VRQ y las VRM se muestran en la Tabla 6.2, y para las regiones VRL se muestran en la Tabla 6.3. 


\begin{tabular}{lrrrrr}
\hline VRM & VRQ & VRM1L & VRM2L & VRM1H & VRM2H \\
\hline Eventos Observados & 718 & 128 & 23 & 423 & 52 \\
\hline Eventos del SM Esperados & $771.25 \pm 110.32$ & $145.30 \pm 27.60$ & $22.90 \pm 9.70$ & $437.25 \pm 82.95$ & $56.99 \pm 10.55$ \\
\hline$e \rightarrow \gamma$ fakes & $23.00 \pm 4.76$ & $4.70 \pm 1.01$ & $1.42 \pm 0.36$ & $12.51 \pm 2.67$ & $2.38 \pm 0.57$ \\
$j \rightarrow \gamma$ fakes & $95.68 \pm 95.34$ & $17.05 \pm 17.04$ & $1.58_{-1.58}^{+1.64}$ & $64.37 \pm 64.15$ & $8.04_{-8.04}^{+8.06}$ \\
$\gamma+$ jets & $565.15 \pm 101.23$ & $106.40 \pm 26.97$ & $15.31 \pm 9.26$ & $304.73 \pm 72.18$ & $30.60 \pm 8.52$ \\
$W \gamma$ & $29.19 \pm 4.27$ & $4.18 \pm 1.27$ & $1.39 \pm 0.75$ & $20.32 \pm 3.15$ & $6.75 \pm 1.69$ \\
$Z(\rightarrow \ell \ell) \gamma$ & $2.28 \pm 0.89$ & $0.30 \pm 0.24$ & $0.14_{-0.14}^{+0.17}$ & $1.17 \pm 0.64$ & $0.30 \pm 0.29$ \\
$Z(\rightarrow \nu \nu) \gamma$ & $10.80 \pm 3.06$ & $1.05 \pm 0.34$ & $0.52 \pm 0.21$ & $6.45 \pm 1.83$ & $2.83 \pm 0.86$ \\
$t \bar{t} \gamma$ & $23.65 \pm 4.21$ & $9.68 \pm 2.35$ & $2.36 \pm 1.01$ & $15.44 \pm 2.82$ & $4.37 \pm 1.09$ \\
$\gamma \gamma / W \gamma \gamma / Z \gamma \gamma$ & $21.50 \pm 5.60$ & $1.95 \pm 1.92$ & $0.17 \pm 0.14$ & $12.26 \pm 3.59$ & $1.73 \pm 0.69$ \\
\hline
\end{tabular}

Tabla 6.2: Estimación del fondo en las regiones de validación VRQ y VRM para los datos de todo Run-2, correspondientes a $139 \mathrm{fb}^{-1}$. Las incertezas son sistemáticas y estadísticas.

\begin{tabular}{lrrrr}
\hline VRL & VRL1 & VRL2 & VRL3 & VRL4 \\
\hline Eventos Observados & 1738 & 259 & 702 & 52 \\
\hline Eventos del SM Esperados & $1724.48 \pm 57.42$ & $261.71 \pm 17.00$ & $731.03 \pm 29.07$ & $50.40 \pm 4.04$ \\
\hline$e \rightarrow \gamma$ fakes & $133.01 \pm 26.54$ & $18.14 \pm 3.77$ & $45.62 \pm 9.65$ & $3.93 \pm 0.90$ \\
$j \rightarrow \gamma$ fakes & $107.24 \pm 106.86$ & $12.84_{-12.84}^{+12.85}$ & $42.29 \pm 42.18$ & $2.12_{-2.12}^{+2.17}$ \\
$\gamma+$ jets & $21.02 \pm 12.01$ & $5.13 \pm 3.93$ & $0.55_{-0.55}^{+0.81}$ & $0.12_{-0.12}^{+0.21}$ \\
$W \gamma$ & $891.08 \pm 98.62$ & $153.21 \pm 19.07$ & $432.51 \pm 47.37$ & $16.87 \pm 2.37$ \\
$Z(\rightarrow \ell \ell) \gamma$ & $59.10 \pm 15.68$ & $12.38 \pm 3.87$ & $7.97 \pm 2.02$ & $0.88 \pm 0.44$ \\
$Z(\rightarrow \nu \nu) \gamma$ & $0.06 \pm 0.05$ & $0.01_{-0.01}^{+0.01}$ & $0.03 \pm 0.02$ & $0.01_{-0.01}^{+0.01}$ \\
$t \bar{t} \gamma$ & $506.34 \pm 59.26$ & $58.65 \pm 7.72$ & $202.06 \pm 23.77$ & $26.47 \pm 3.67$ \\
$\gamma \gamma / W \gamma \gamma / Z \gamma \gamma$ & $6.63 \pm 2.39$ & $1.34 \pm 0.47$ & $0.00 \pm 0.00$ & $0.00_{-0.00}^{+0.00}$ \\
\hline
\end{tabular}

Tabla 6.3: Estimación del fondo en las regiones de validación VRL para los datos de todo Run-2, correspondientes a $139 \mathrm{fb}^{-1}$. Las incertezas son sistemáticas y estadísticas.

Las distribuciones observadas en VRQ, VRM1L, VRM2L, VRM1H y VRM2H se muestran en las Figuras 6.4, 6.5, 6.6, 6.7 y 6.8, respectivamente, para el conjunto de datos de todo el Run-2. De forma similar, las distribuciones para VRL1, VRL2, VRL3, VRL4 se muestran en las Figuras 6.9, 6.10, 6.11 y 6.12, respectivamente, para el conjunto de datos de todo el Run-2.

De forma general se encuentra un buen acuerdo entre los datos y la predicción del fondo para todas las regiones de validación del análisis. Sin embargo, en particular para VRQ y VRM1L (véase la Tabla 6.2 y las Figuras 6.4 y 6.5), se observa una deficiencia de datos con respecto al fondo, que es cubierto al considerar las incertezas estadísticas y sistemáticas.

Para el caso de la región de validación dominada por el fondo $e \rightarrow \gamma$ fakes (VRE), la Tabla 6.4 y la Figura 6.13 muestran que la predicción de fondo está en buen acuerdo con los datos observados. La región de validación tiene $\sim 75 \%$ de pureza en el fondo correspondiente y, a pesar de estar lo más cerca posible de las SR, tiene una contaminación de señal muy baja $(<1 \%)$, como se muestra en la Figura 5.17.

Una forma de ver el buen acuerdo encontrado entre las predicciones y los datos es con los pulls para las distintas regiones de validación, como se muestra en la Figura 6.14. Los pulls se definen como la resta entre los eventos esperados y observados (en una dada región), sobre la incerteza total (estadística y sistemática). En esta figura se agregan también las regiones de control (que, por construcción, luego del 


\begin{tabular}{lr}
\hline Fakes VR & VRE \\
\hline Eventos Observados & 500 \\
\hline Eventos del SM Esperados & $529.99 \pm 103.96$ \\
\hline$e \rightarrow \gamma$ fakes & $361.43 \pm 74.89$ \\
$j \rightarrow \gamma$ fakes & $79.90 \pm 79.64$ \\
$\gamma+$ jets & $8.83 \pm 7.84$ \\
$W \gamma$ & $51.40 \pm 11.01$ \\
$Z(\rightarrow \ell \ell) \gamma$ & $0.51 \pm 0.37$ \\
$Z(\rightarrow \nu \nu) \gamma$ & $4.93 \pm 1.62$ \\
$t \bar{t} \gamma$ & $22.63 \pm 3.61$ \\
$\gamma \gamma / W \gamma \gamma / Z \gamma \gamma$ & $0.35 \pm 0.07$ \\
\hline
\end{tabular}

Tabla 6.4: Estimación del fondo en la región de validación VRE para los datos de todo Run-2, correspondientes a $139 \mathrm{fb}^{-1}$. Las incertezas son sistemáticas y estadísticas.

ajuste la diferencia de eventos esperados y observados es cero), y solo los eventos esperados para las regiones de señal, porque aquí aún no pueden verse los datos del Run-2 completo (se dice que estas regiones están blinded).En este resumen se aprecia un buen acuerdo entre los datos y las predicciones de fondo del SM en todas las regiones de validación, validando el método y permitiendo analizar las regiones de señal como se hace en la próxima sección.

\subsubsection{Resultados en las regiones de señal}

Luego de constatar el buen acuerdo entre las predicciones de fondo y las mediciones en las regiones de validación, como se hizo en la sección anterior, se estudia la estimación del fondo del SM para las diferentes regiones de búsqueda de señal de nueva física usadas en la presente tesis. El resultado se muestra en la Tabla 6.5 para el conjunto de datos de todo Run-2, donde se omiten los valores de los datos observados ya que en este momento están en el proceso de aprobación dentro de la colaboración ATLAS para su publicación. En la sección siguiente se presentan los datos observados para una porción de los mismos, que fue aprobada puntualmente para la presentación de esta tesis. El número de eventos esperado a partir de procesos del SM en las regiones SRL, SRM y SRH es de $2.94 \pm 1.2,2.91 \pm 0.97$ y $2.63 \pm 0.85$, respectivamente.

El detalle de las incertezas sistemáticas dominantes en la estimación del fondo para las diferentes regiones de señal se muestran en la Tabla 6.6 donde, debido a la posible correlación entre las distintas componentes, la incerteza total no es necesariamente la suma en cuadratura de éstas. Se desprende de esta tabla que las incertezas sistemáticas teóricas son las dominantes, seguidas por las de los métodos data-driven y las experimentales asociadas con los jets.

Las distribuciones para $E_{\mathrm{T}}^{\text {miss }}$, con todos los cortes de selección de cada una de las regiones de señal excepto en la propia variable $E_{\mathrm{T}}^{\text {miss }}$, para cada una de las regiones de señal, se muestran en la Figura 6.16. Los datos solo se muestran en los bines que no pertenecen a las SR, pero la estimación del fondo se presenta en todo el rango. Se agregan además, las distribuciones para dos modelos de señal con masas de gluino y neutralino para las cuales fueron optimizadas cada una de las regiones, a modo de comparación. 


\begin{tabular}{lrrr}
\hline Regiones de Señal & SRL & SRM & SRH \\
\hline Eventos Observados & - & - & - \\
\hline Eventos del SM Esperados & $2.94 \pm 1.20$ & $2.91 \pm 0.97$ & $2.63 \pm 0.85$ \\
\hline$e \rightarrow \gamma$ fakes & $0.18 \pm 0.09$ & $0.05 \pm 0.03$ & $0.07 \pm 0.04$ \\
$j \rightarrow \gamma$ fakes & $0.30_{-0.30}^{+0.35}$ & $0.28_{-0.28}^{+0.33}$ & $0.19_{-0.19}^{+0.23}$ \\
$\gamma+$ jets & $0.48_{-0.48}^{+0.53}$ & $0.19_{-0.19}^{+0.20}$ & $0.06_{-0.06}^{+0.06}$ \\
$W \gamma$ & $0.65_{-0.65}^{+0.86}$ & $0.81 \pm 0.70$ & $1.01 \pm 0.69$ \\
$Z(\rightarrow \ell \ell) \gamma$ & $0.03_{-0.03}^{+0.10}$ & $0.03_{-0.03}^{+0.04}$ & $0.00_{-0.00}^{+0.01}$ \\
$Z(\rightarrow \nu \nu) \gamma$ & $0.39 \pm 0.17$ & $0.42 \pm 0.18$ & $1.08 \pm 0.37$ \\
$t \bar{t} \gamma$ & $0.67 \pm 0.37$ & $0.88 \pm 0.44$ & $0.22 \pm 0.17$ \\
$\gamma \gamma / W \gamma \gamma / Z \gamma \gamma$ & $0.24 \pm 0.12$ & $0.24 \pm 0.12$ & $0.00 \pm 0.00$ \\
\hline
\end{tabular}

Tabla 6.5: Estimación del fondo en las regiones de señal SRL, SRM y SRH para las muestras de todo Run-2, correspondientes a $139 \mathrm{fb}^{-1}$. Las incertezas incluidas son tanto estadísticas como sistemáticas. 

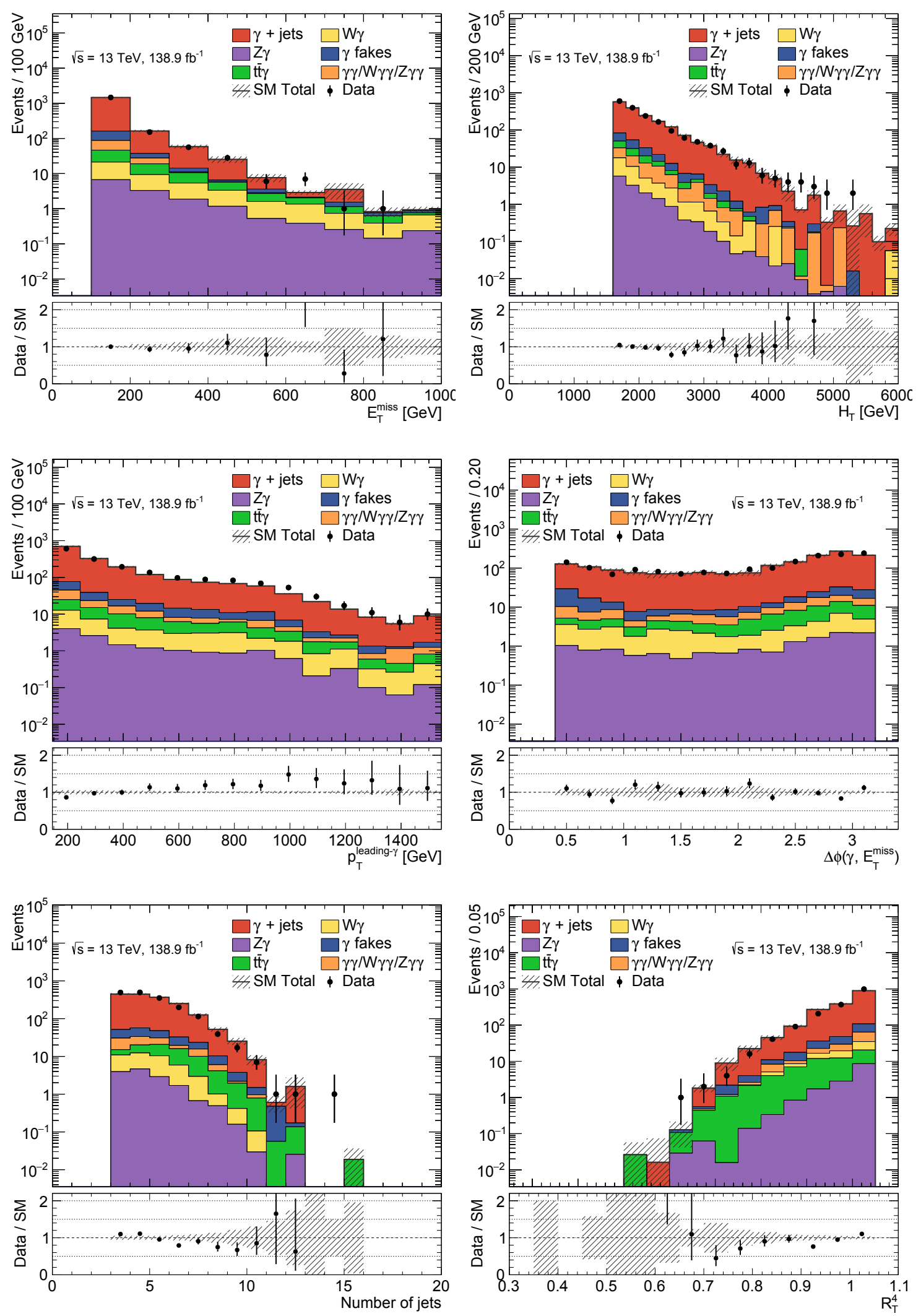

Figura 6.1: Distribuciones observadas en la región de control CRQ después del ajuste de solo-fondo para los datos de todo Run-2, correspondientes a $139 \mathrm{fb}^{-1}$. Las incertezas en los fondos del SM son sólo estadísticas. 

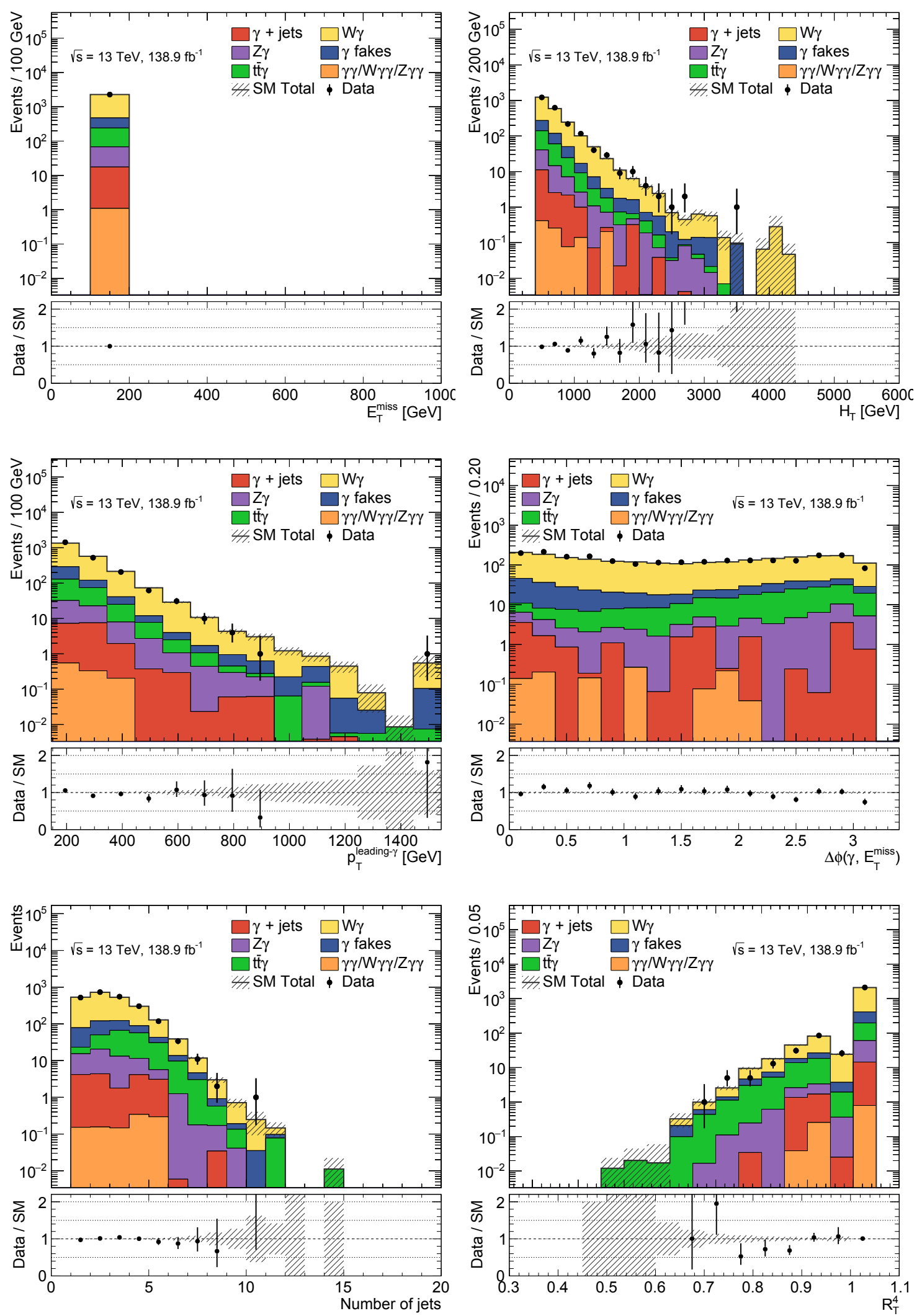

Figura 6.2: Distribuciones observadas en la región de control CRW después del ajuste de solo-fondo para los datos de todo Run-2, correspondientes a $139 \mathrm{fb}^{-1}$. Las incertezas en los fondos del SM son sólo estadísticas. 

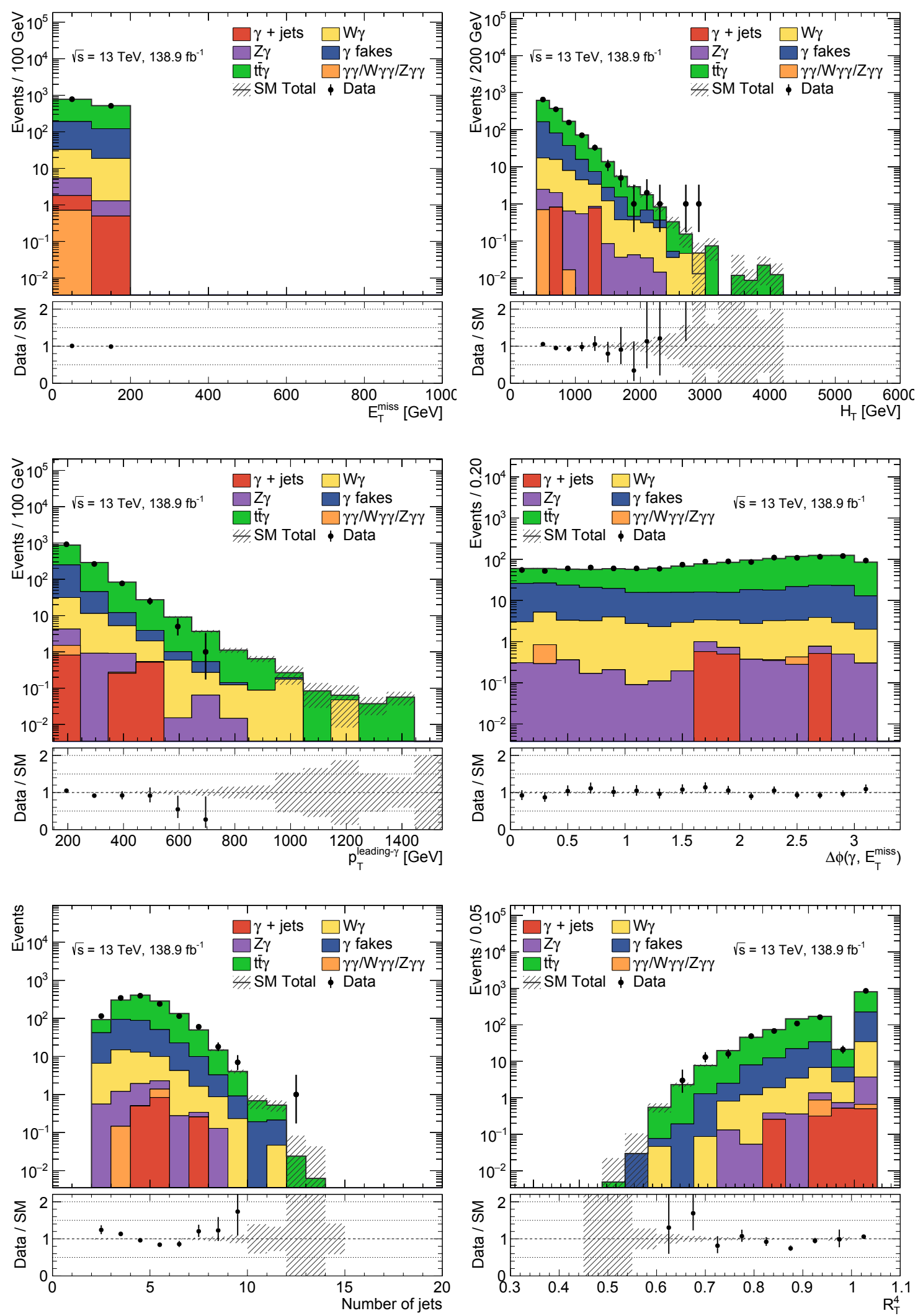

Figura 6.3: Distribuciones observadas en la región de control CRT después del ajuste de solo-fondo para los datos de todo Run-2, correspondientes a $139 \mathrm{fb}^{-1}$. Las incertezas en los fondos del SM son sólo estadísticas. 

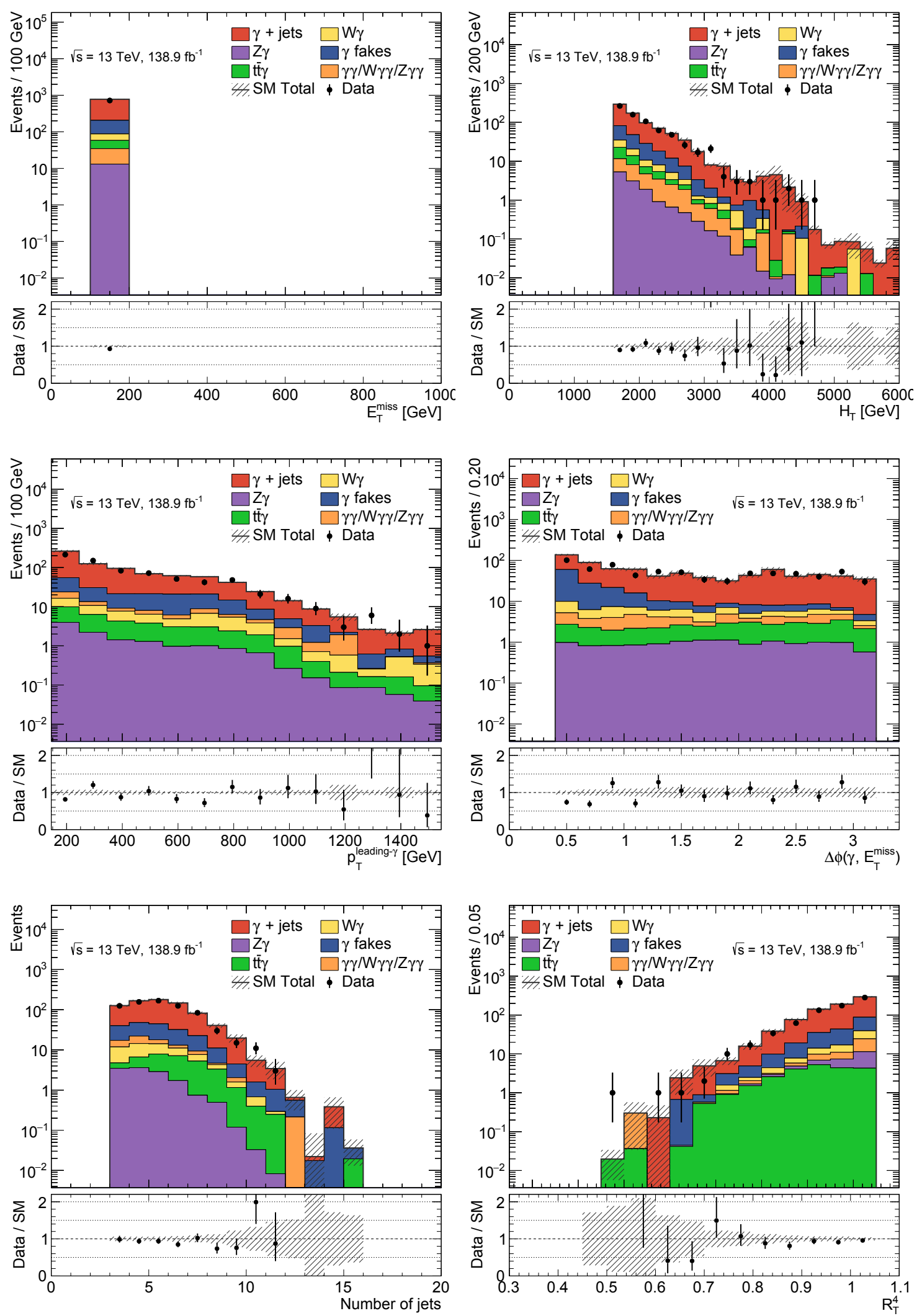

Figura 6.4: Distribuciones observadas en la región de validación VRQ después del ajuste de solo-fondo para los datos de todo Run-2, correspondientes a $139 \mathrm{fb}^{-1}$. Las incertezas en los fondos del SM son sólo estadísticas. 

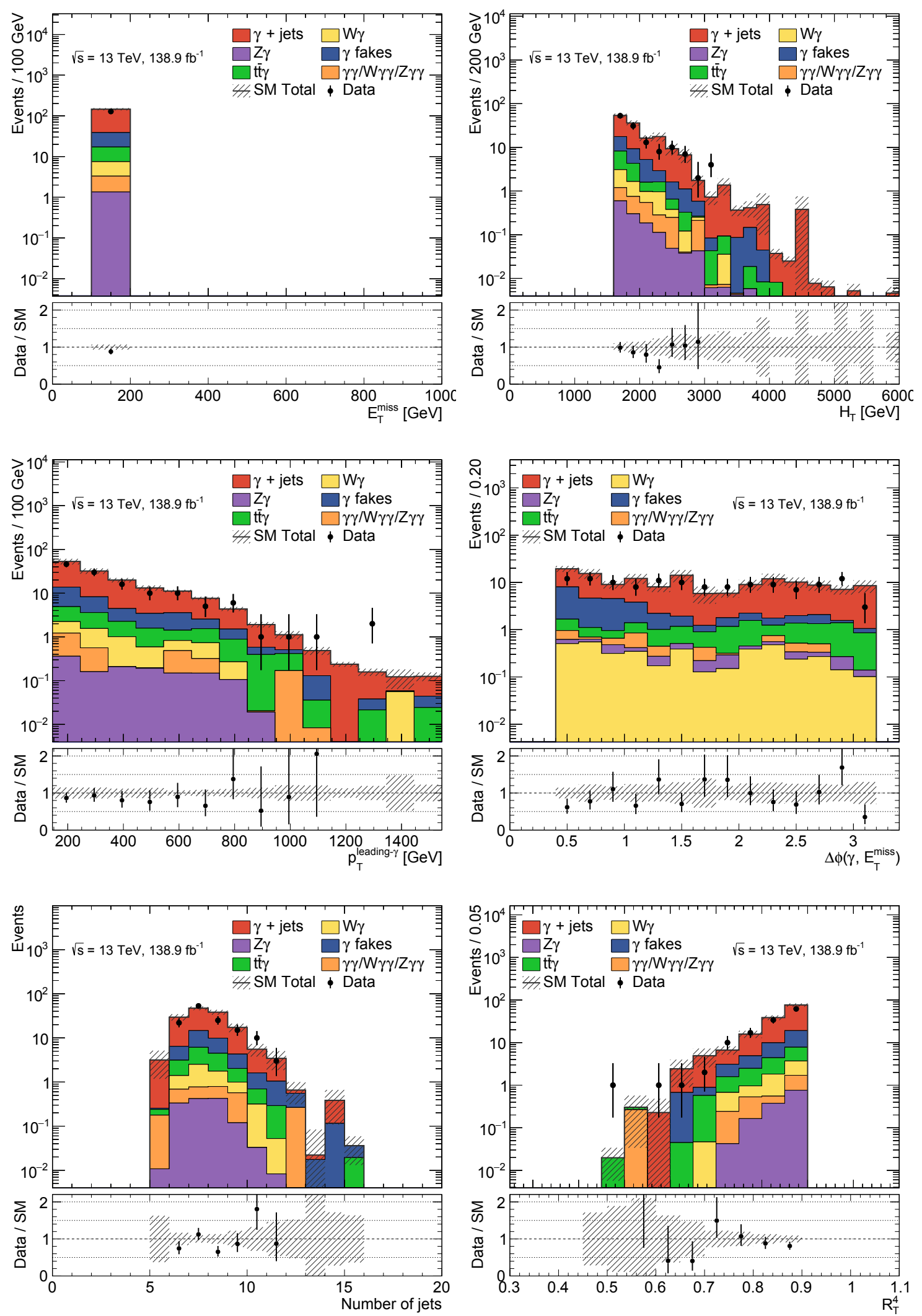

Figura 6.5: Distribuciones observadas en la región de validación VRM1L después del ajuste de solo-fondo para los datos de todo Run-2, correspondientes a $139 \mathrm{fb}^{-1}$. Las incertezas en los fondos del SM son sólo estadísticas. 

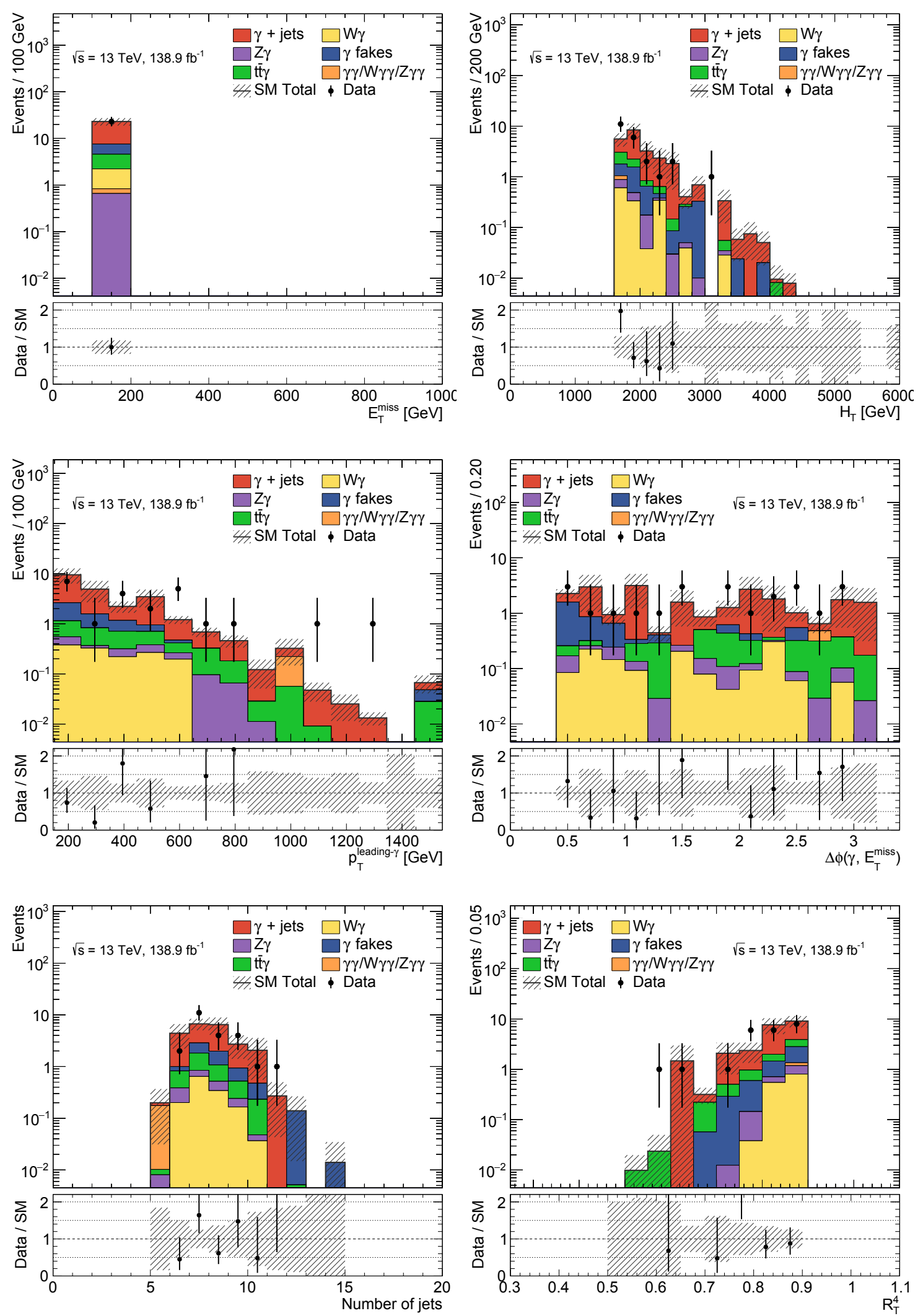

Figura 6.6: Distribuciones observadas en la región de validación VRM2L después del ajuste de solo-fondo para los datos de todo Run-2, correspondientes a $139 \mathrm{fb}^{-1}$. Las incertezas en los fondos del SM son sólo estadísticas. 

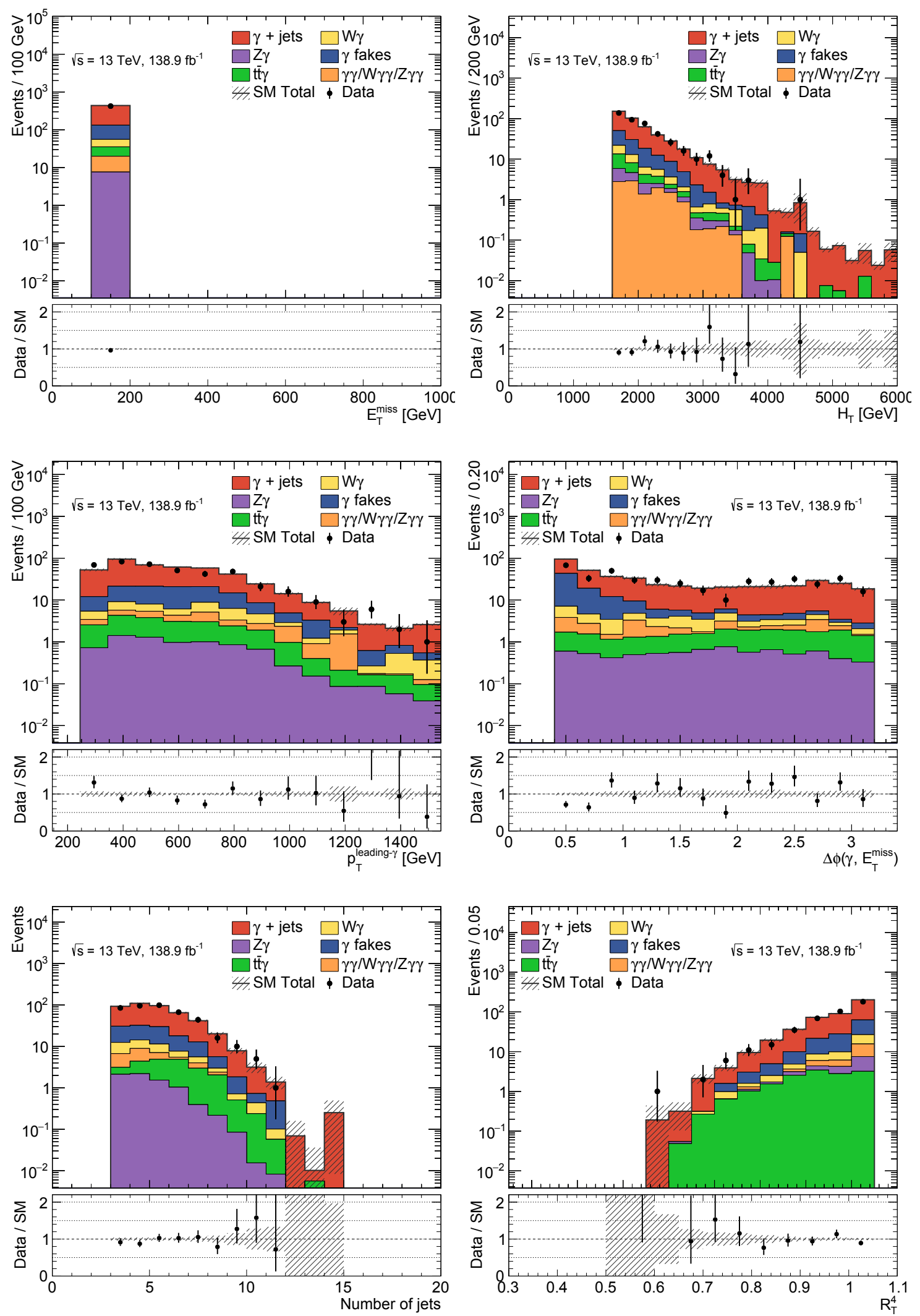

Figura 6.7: Distribuciones observadas en la región de validación VRM1H después del ajuste de solo-fondo para los datos de todo Run-2, correspondientes a $139 \mathrm{fb}^{-1}$. Las incertezas en los fondos del SM son sólo estadísticas. 

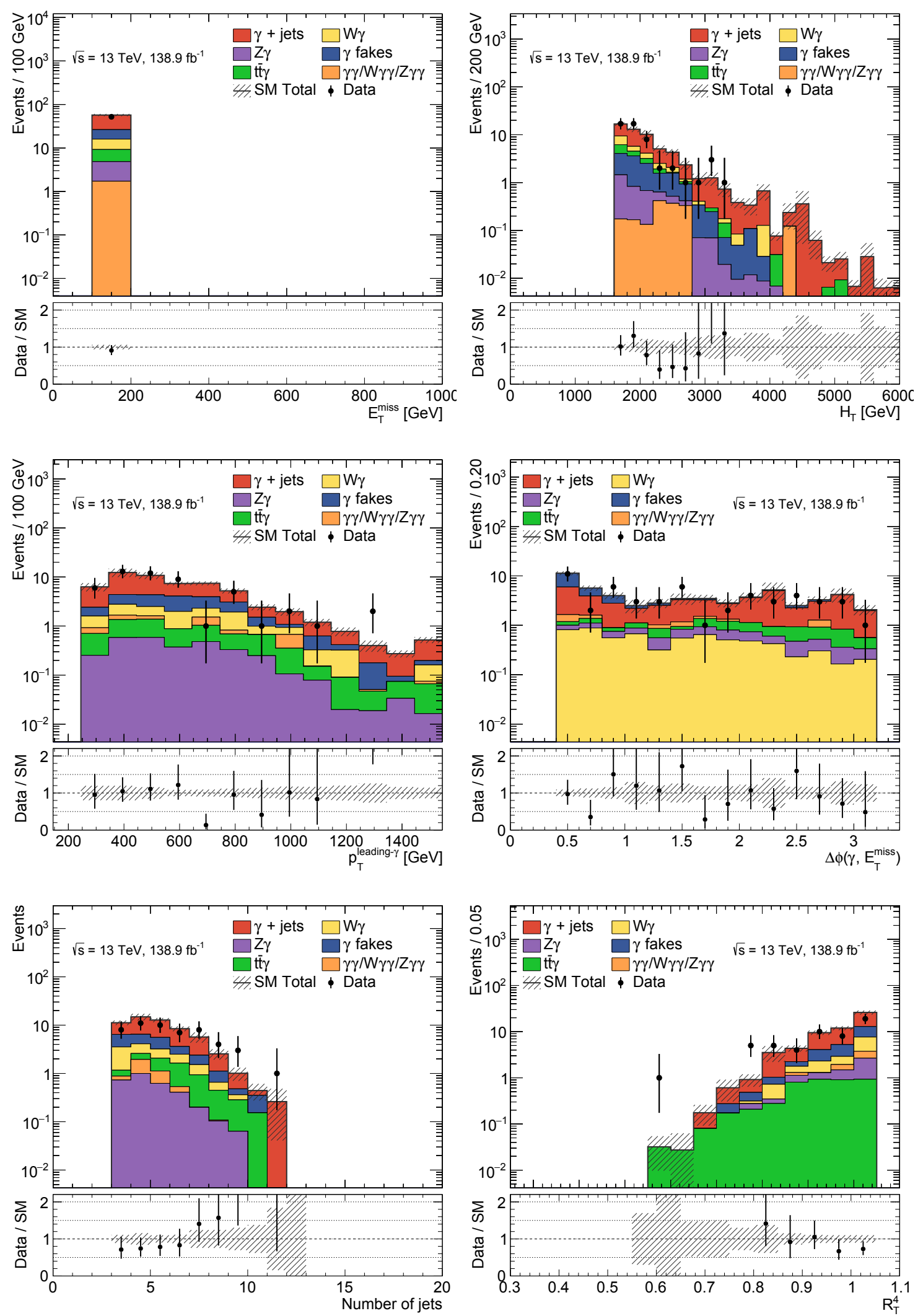

Figura 6.8: Distribuciones observadas en la región de validación VRM2H después del ajuste de solo-fondo para los datos de todo Run-2, correspondientes a $139 \mathrm{fb}^{-1}$. Las incertezas en los fondos del SM son sólo estadísticas. 

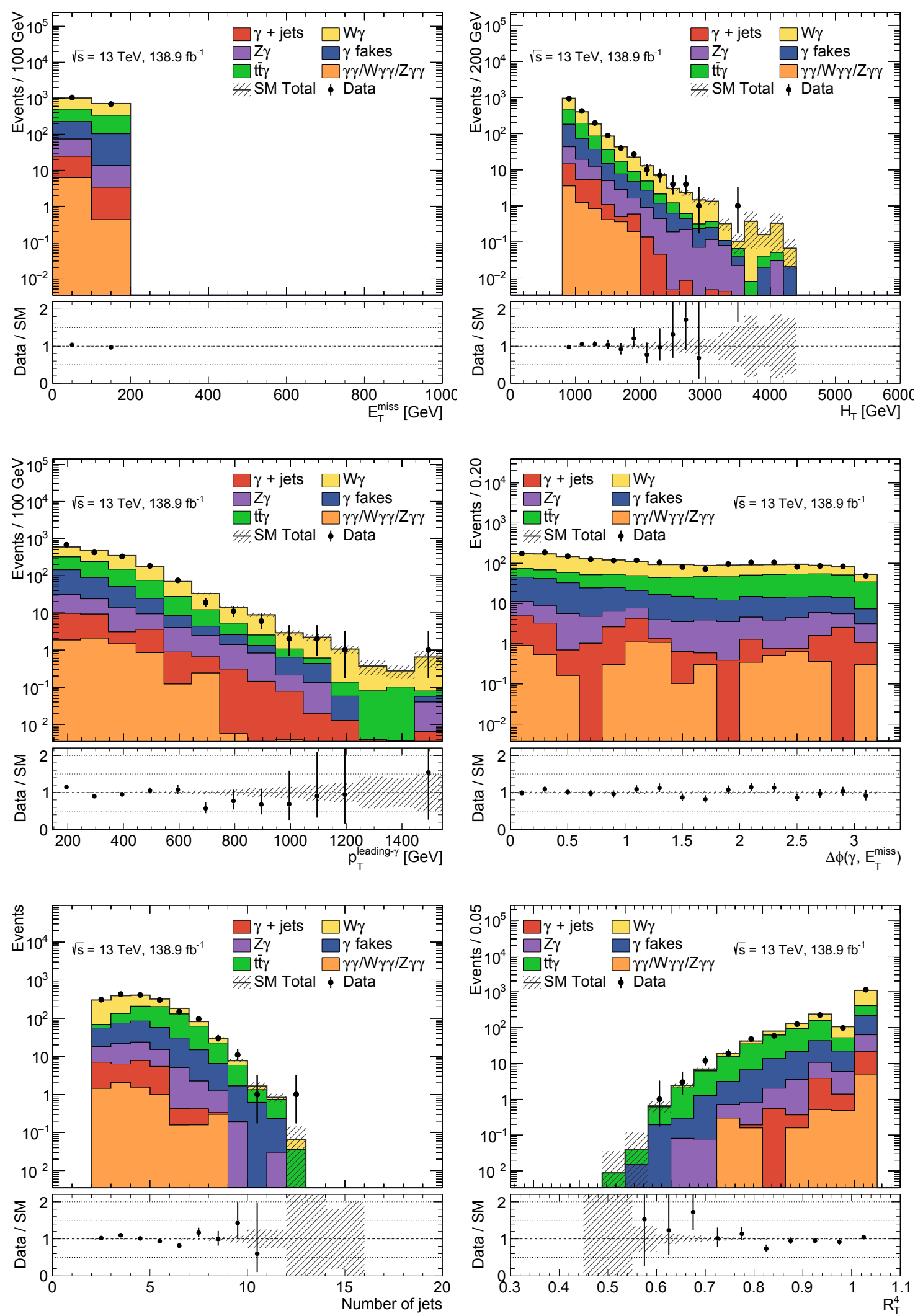

Figura 6.9: Distribuciones observadas en la región de validación VRL1 después del ajuste de solo-fondo para los datos de todo Run-2, correspondientes a $139 \mathrm{fb}^{-1}$. Las incertezas en los fondos del SM son sólo estadísticas. 

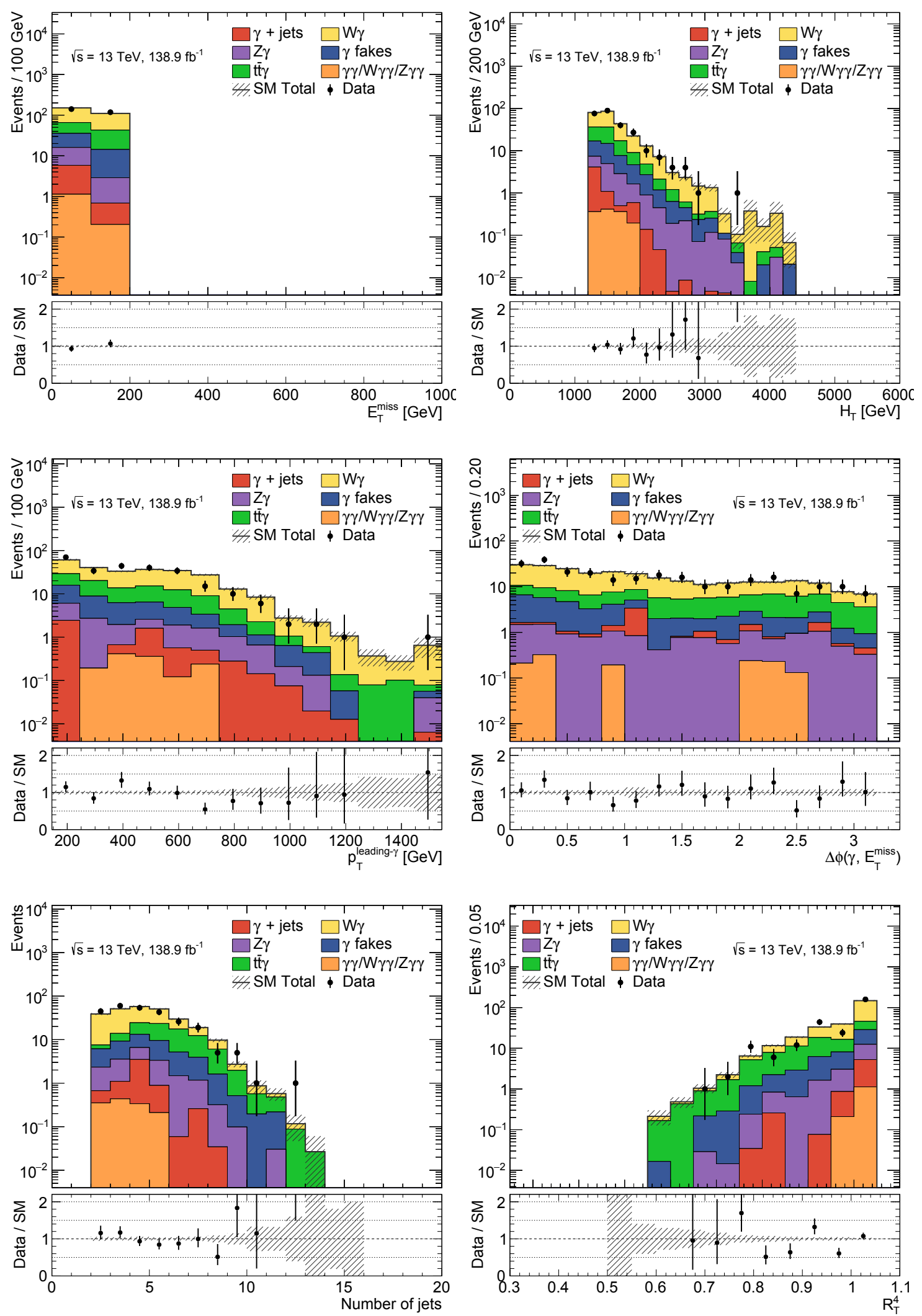

Figura 6.10: Distribuciones observadas en la región de validación VRL2 después del ajuste de solo-fondo para los datos de todo Run-2, correspondientes a $139 \mathrm{fb}^{-1}$. Las incertezas en los fondos del SM son sólo estadísticas. 

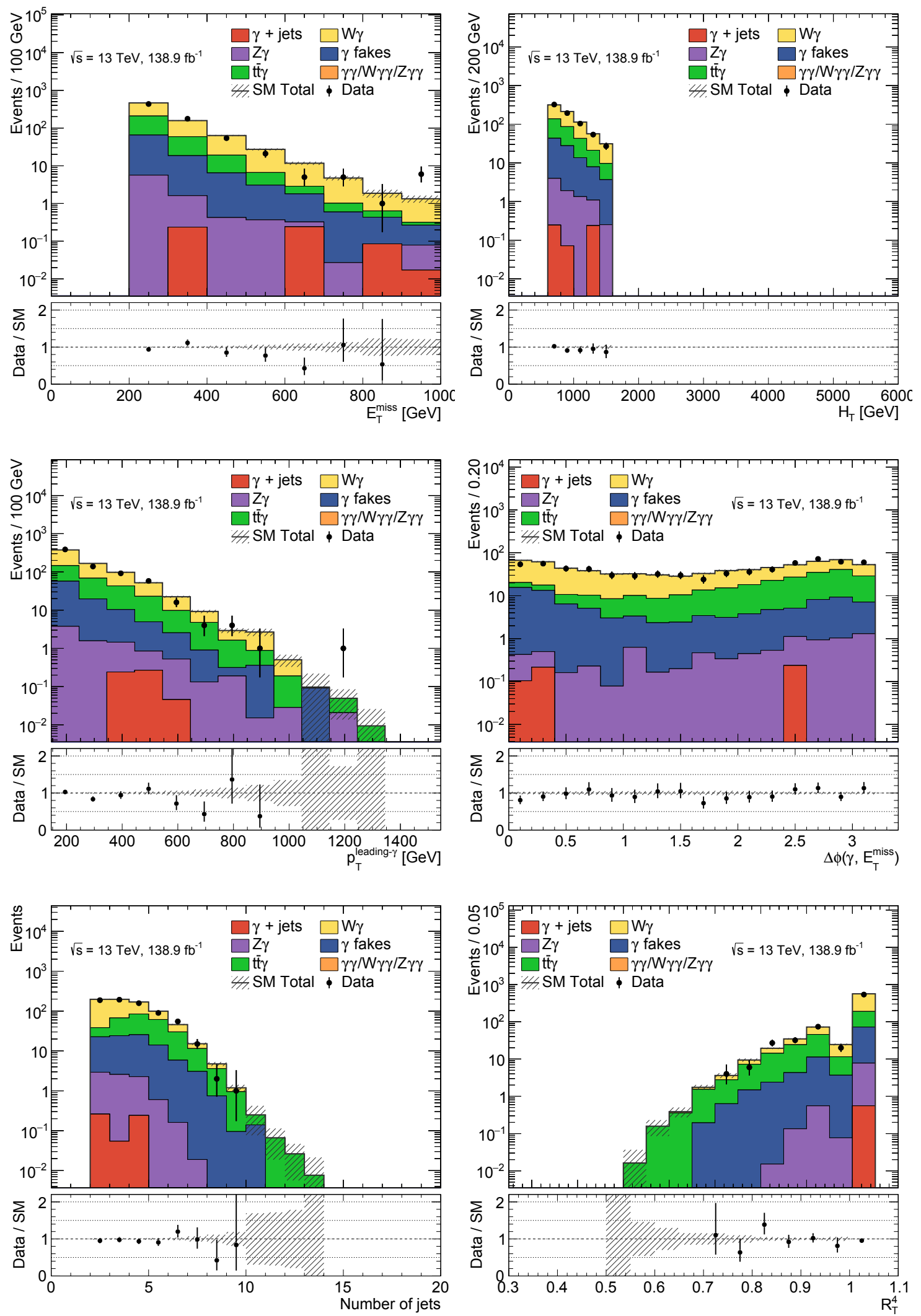

Figura 6.11: Distribuciones observadas en la región de validación VRL3 después del ajuste de solo-fondo para los datos de todo Run-2, correspondientes a $139 \mathrm{fb}^{-1}$. Las incertezas en los fondos del SM son sólo estadísticas. 

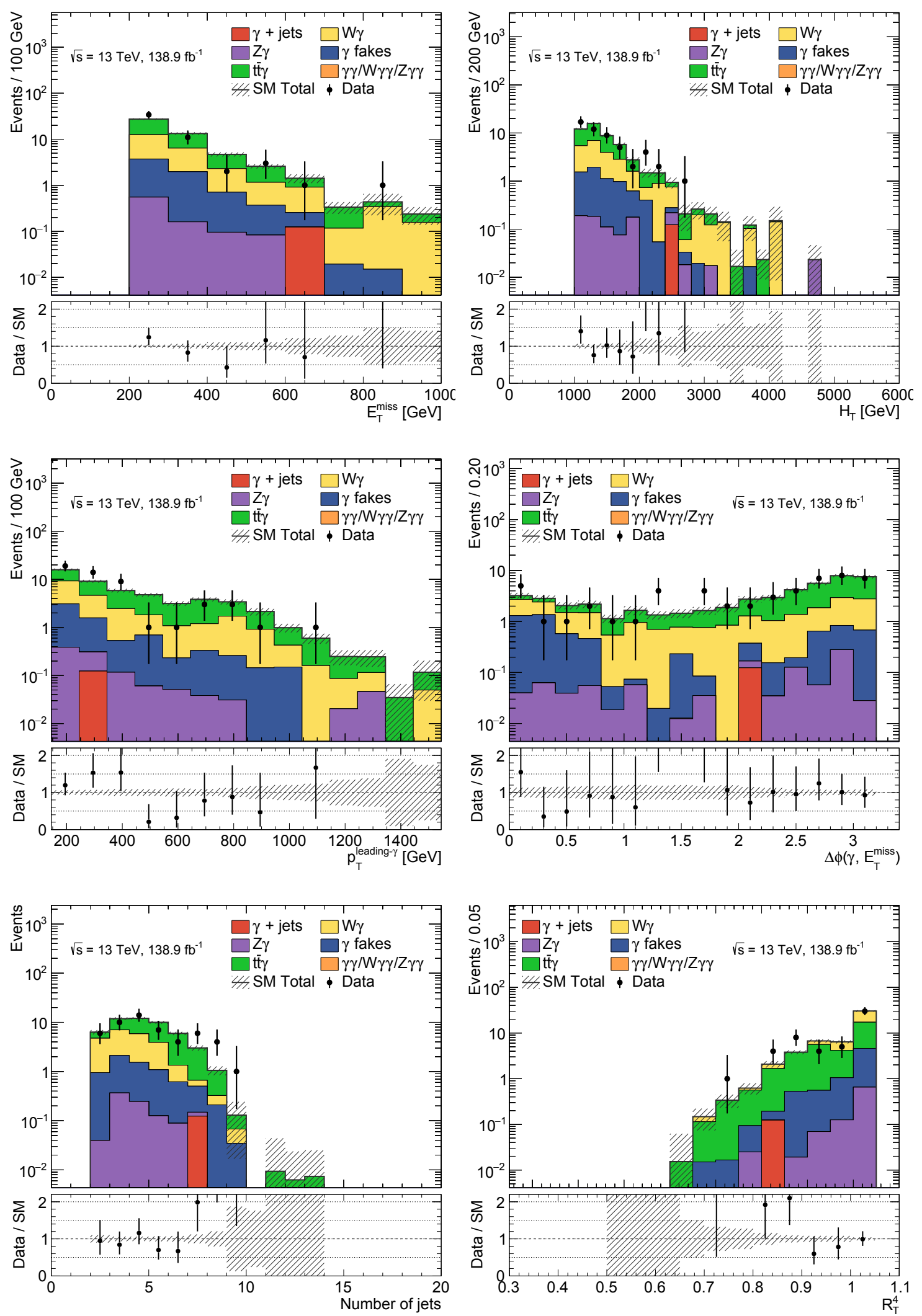

Figura 6.12: Distribuciones observadas en la región de validación VRL4 después del ajuste de solo-fondo para los datos de todo Run-2, correspondientes a $139 \mathrm{fb}^{-1}$. Las incertezas en los fondos del SM son sólo estadísticas. 

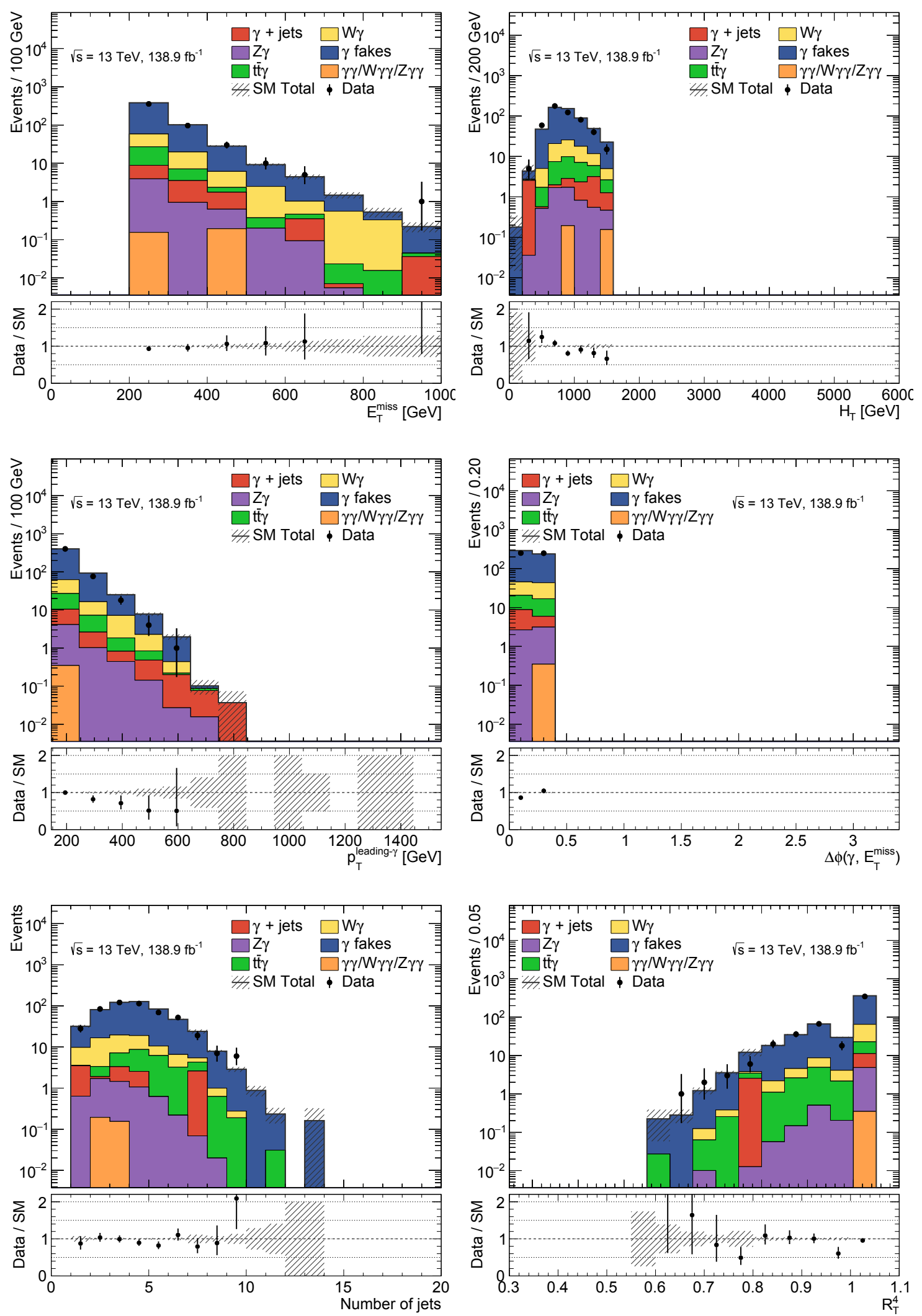

Figura 6.13: Distribuciones observadas en la región de validación VRE para los datos de todo Run-2, correspondientes a $139 \mathrm{fb}^{-1}$. Las incertezas en los fondos del SM son sólo estadísticas. 


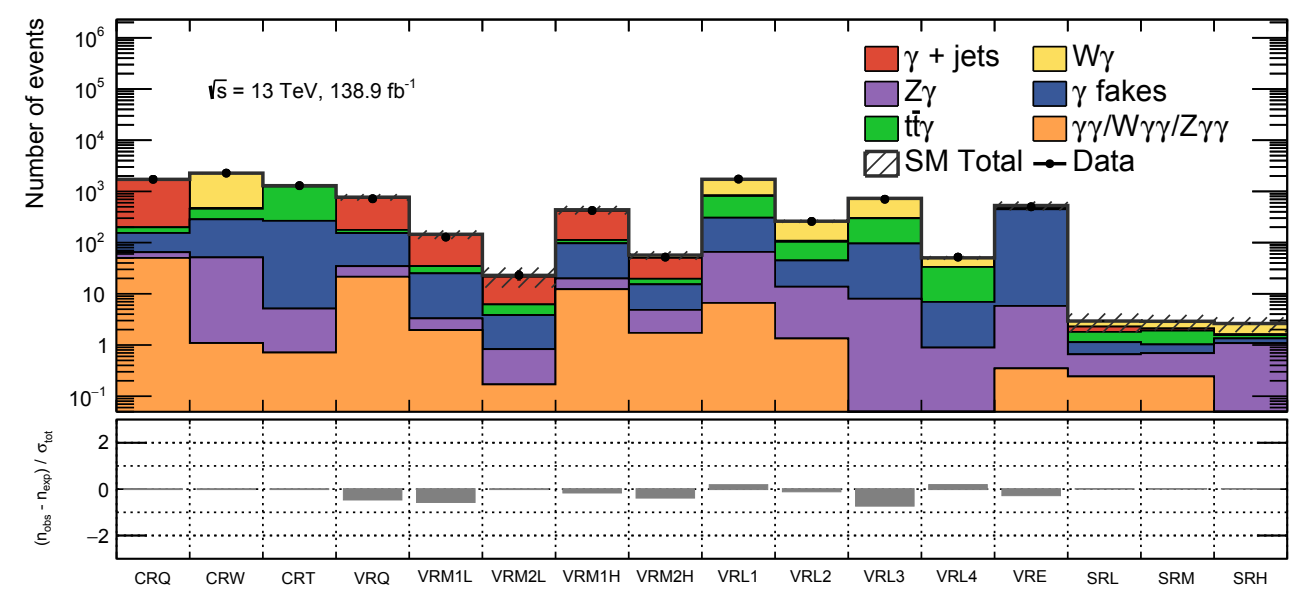

Figura 6.14: El número de eventos observados y esperados en las regiones de control, validación y señal (blinded), para el conjunto de datos de todo Run-2, correspondientes a $139 \mathrm{fb}^{-1}$. El panel inferior muestra la diferencia, en desviaciones estándar, entre el número de eventos observados y esperados (pulls). El pull se calcula utilizando las incertezas sistemáticas y estadísticas en la predicción del fondo $\left(\sigma_{\text {tot }}\right)$. 

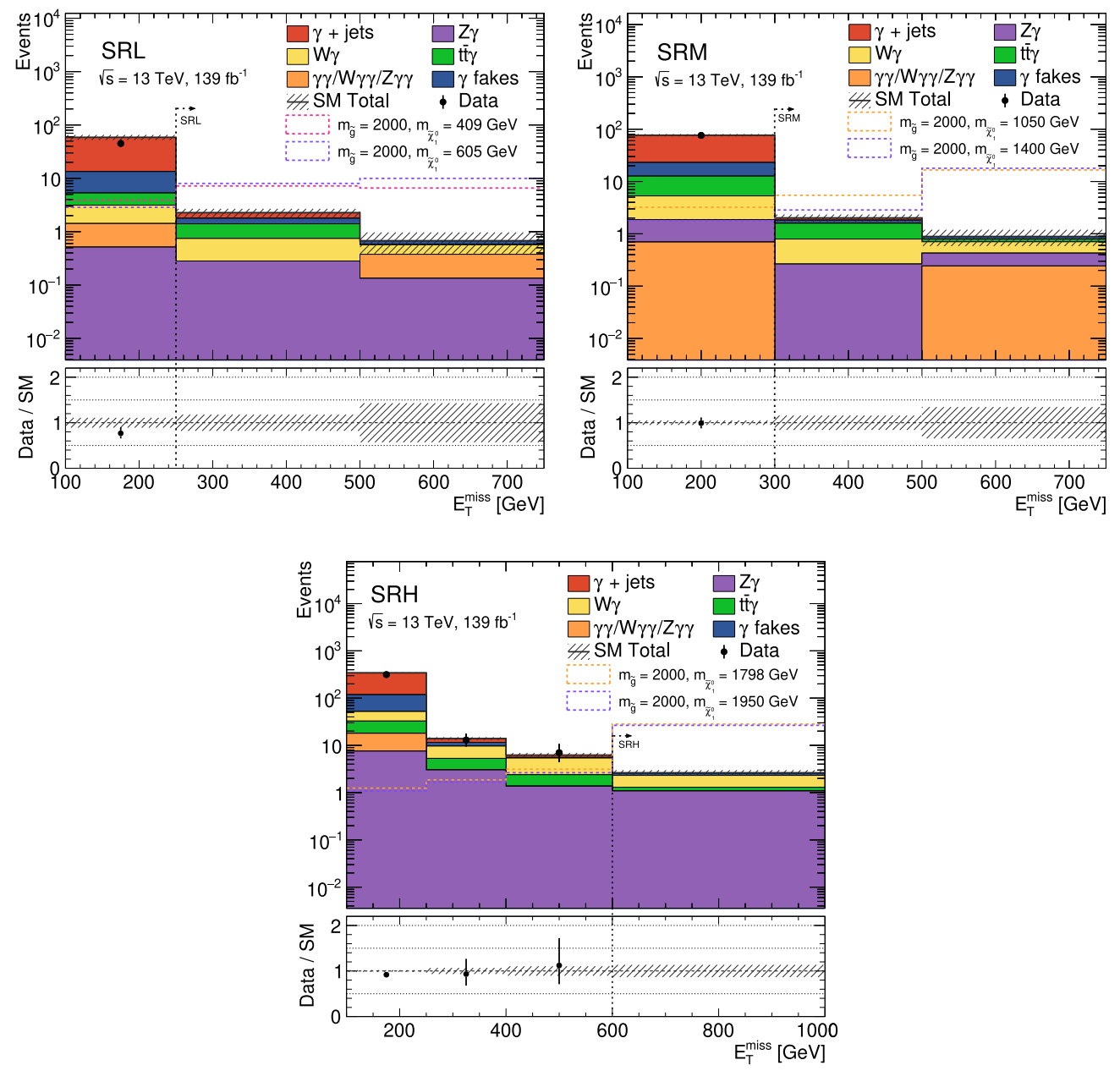

Figura 6.15: Distribuciones observadas blinded (puntos con barras de incerteza) y fondo esperado (histogramas sólidos) $E_{\mathrm{T}}^{\text {miss }}$ en las regiones de señal SRL, SRM y $\mathrm{SRH}$, luego del ajuste de solo-fondo. Las distribuciones para dos modelos de señal, con los valores especificados para las masas de gluinos y neutralinos se agregan para comparación. Las incertezas en los fondos del SM son sólo estadísticas. 
Tabla 6.6: Resumen de las incertezas sistemáticas dominantes en la estimación del fondo total en las tres SR. Debido a la posible correlación entre componentes, la incerteza total no es necesariamente la suma en cuadratura de éstas.

\begin{tabular}{|c|c|}
\hline Incertezas & SRL \\
\hline Fondo total esperado & 2.94 \\
\hline Total estadístico $\left(\sqrt{N_{\exp }}\right)$ & \pm 1.71 \\
\hline Total sistemático de fondo & $\pm 1.20[40.74 \%]$ \\
\hline$W \gamma$ theo. syst. & $\pm 0.56[19.0 \%]$ \\
\hline$\gamma+$ jets theo. syst. & $\pm 0.48[16.5 \%]$ \\
\hline JET_JER_EffectiveNP_1 & $\pm 0.47[15.8 \%]$ \\
\hline JET_JER_EffectiveNP_2 & $\pm 0.36[12.2 \%]$ \\
\hline jet $\rightarrow \gamma$ fakes syst. & $\pm 0.30[10.2 \%]$ \\
\hline$t \bar{t} \gamma$ theo. syst. & $\pm 0.30[10.1 \%]$ \\
\hline JET_Flavor_Composition & $\pm 0.23[7.7 \%]$ \\
\hline JET_JER_EffectiveNP_6 & $\pm 0.20[6.8 \%]$ \\
\hline JET_JER_EffectiveNP_7 & $\pm 0.20[6.7 \%]$ \\
\hline JET_EffectiveNP_Modelling1 & $\pm 0.20[6.7 \%]$ \\
\hline Incertezas & SRM \\
\hline Fondo total esperado & 2.91 \\
\hline Total estadístico $\left(\sqrt{N_{\exp }}\right)$ & \pm 1.70 \\
\hline Total sistemático de fondo & $\pm 0.97[33.22 \%]$ \\
\hline $\bar{~} W \gamma$ theo. syst. & 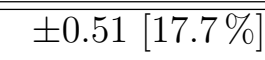 \\
\hline$t \bar{t} \gamma$ theo. syst. & $\pm 0.39[13.4 \%]$ \\
\hline jet $\rightarrow \gamma$ fakes syst. & $\pm 0.28[9.7 \%]$ \\
\hline JET_Flavor_Response & $\pm 0.18[6.1 \%]$ \\
\hline$\gamma+\overline{\text { jets theo. syst. }}$ & $\pm 0.16[5.6 \%]$ \\
\hline$Z \nu \nu \gamma$ theo. syst. & $\pm 0.16[5.5 \%]$ \\
\hline JET_EtaIntercalibration_Modelling & $\pm 0.15[5.3 \%]$ \\
\hline JET_Pileup_OffsetMu & $\pm 0.15[5.2 \%]$ \\
\hline JET_EffectiveNP_Modelling1 & $\pm 0.14[4.9 \%]$ \\
\hline JET_EtaIntercalibration_TotalStat & $\pm 0.14[4.8 \%]$ \\
\hline Incertezas & SRH \\
\hline Fondo total esperado & 2.63 \\
\hline Total estadístico $\left(\sqrt{N_{\text {exp }}}\right)$ & \pm 1.62 \\
\hline Total sistemático de fondo & $\pm 0.85[32.26 \%]$ \\
\hline$W \gamma$ theo. syst. & $\pm 0.72[27.3 \%]$ \\
\hline$Z \nu \nu \gamma$ theo. syst. & $\pm 0.32[12.3 \%]$ \\
\hline jet $\rightarrow \gamma$ fakes syst. & $\pm 0.19[7.1 \%]$ \\
\hline$t \bar{t} \gamma$ theo. syst. & $\pm 0.16[6.1 \%]$ \\
\hline FT efficiency (Light) & $\pm 0.09[3.5 \%]$ \\
\hline$e / \gamma$ scale & $\pm 0.08[3.1 \%]$ \\
\hline JET_Pileup_RhoTopology & $\pm 0.08[2.9 \%]$ \\
\hline JET_EffectiveNP_Modelling1 & $\pm 0.07[2.7 \%]$ \\
\hline JET_Flavor_Composition & $\pm 0.07[2.7 \%]$ \\
\hline FT efficiency extrapolation & $\pm 0.06[2.3 \%]$ \\
\hline
\end{tabular}




\subsection{Resultados en las regiones de señal para datos de 2015 y 2016}

Con el fin de presentar resultados en las regiones de señal en este trabajo, además de la potencialidad de búsqueda y capacidad de determinar límites en la teoría, se acordó con la colaboración mostrar los resultados en base a los datos colectados en 2015 y 2016 (aunque no han sido aprobados oficialmente para su exposición pública). En la Tabla 6.7 se muestra la comparación de datos observados y la correspondiente estimación de fondo para las regiones SRL, SRM y SRH. El número de eventos esperados a partir de procesos del SM en dichas regiones es de $0.66 \pm 0.59,0.61 \pm 0.55$ y $0.66 \pm 0.27$, respectivamente, mientras que el número de eventos observados es 2 en SRL, 0 en SRM y 5 en SRH. Se encuentra que la fuerte optimización realizada para el conjunto de datos de todo el Run-2 en las regiones de señal (Sección 5.3.1), remueve la mayoría del fondo del SM en las mismas, para el subconjunto de datos aquí considerados. Los cómputos de compatibilidad y los límites correspondientes en los valores de masa de gluinos y neutralinos se detallan a continuación.

\begin{tabular}{lrrr}
\hline Signal Regions & SRL & SRM & SRH \\
\hline Observed events & 2 & 0 & 5 \\
\hline Expected SM events & $0.66 \pm 0.59$ & $0.61 \pm 0.55$ & $0.66 \pm 0.27$ \\
\hline$e \rightarrow \gamma$ fakes & $0.03 \pm 0.03$ & $0.01_{-0.01}^{+0.02}$ & $0.03 \pm 0.02$ \\
$j \rightarrow \gamma$ fakes & $0.09_{-0.09}^{+0.13}$ & $0.09_{-0.09}^{+0.13}$ & $0.05_{-0.05}^{+0.05}$ \\
$\gamma+$ jets & $0.08 \pm 0.08$ & $0.08 \pm 0.07$ & $0.02_{-0.02}^{+0.02}$ \\
$W \gamma$ & $0.20_{-0.20}^{+0.53}$ & $0.16_{-0.16}^{+0.51}$ & $0.26 \pm 0.19$ \\
$Z(\rightarrow \ell \ell) \gamma$ & $0.00 \pm 0.00$ & $0.00 \pm 0.00$ & $0.00 \pm 0.00$ \\
$Z(\rightarrow \nu \nu) \gamma$ & $0.09 \pm 0.05$ & $0.06 \pm 0.03$ & $0.27 \pm 0.12$ \\
$t \bar{t} \gamma$ & $0.10 \pm 0.09$ & $0.20 \pm 0.12$ & $0.04_{-0.04}^{+0.04}$ \\
$\gamma \gamma / W \gamma \gamma / Z \gamma \gamma$ & $0.07 \pm 0.02$ & $0.00 \pm 0.00$ & $0.00 \pm 0.00$ \\
\hline
\end{tabular}

Tabla 6.7: Estimación del fondo en las regiones de señal SRL, SRM y SRH para el conjunto de datos de $2015+2016$, correspondientes a $36.2 \mathrm{fb}^{-1}$. Las incertezas incluidas son tanto estadísticas como sistemáticas.

Las distribuciones para $E_{\mathrm{T}}^{\text {miss }}$ para cada una de las regiones de señal con todos los cortes de selección correspondientes (excepto en la propia variable $E_{\mathrm{T}}^{\text {miss }}$ ), se muestran en la Figura 6.16. A modo de comparación, se agregan además las distribuciones para dos modelos de señal con masas de gluino y neutralino en el rango para el cual cada SR fue optimizada.

Por último, se muestran también los pulls correspondientes a los datos de 36.2 $f b^{-1}$ (Figura 6.17), donde ahora se agregan los datos en las regiones de señal. De igual manera que para el estudio realizado para todo el Run-2, se observa un buen acuerdo entre la predicción del fondo y los datos observados en todas las regiones de validación. Un "exceso" de datos con respecto al fondo esperado, estimado en $\sim 3 \sigma$, se observa en la región SRH. Debido a que este resultado puede ser consecuencia de fluctuaciones estadísticas, potenciadas quizás por el bajo fondo esperado al considerar solo parte de los datos del Run-2, es importante esperar a observar los resultados con la estadística completa del análisis (todo Run-2) una vez que sean aprobados por la colaboración, evitando así discusiones o interpretaciones anticipadas al resultado final. Teniendo en cuenta los lineamientos de ATLAS en relación a la significancia 

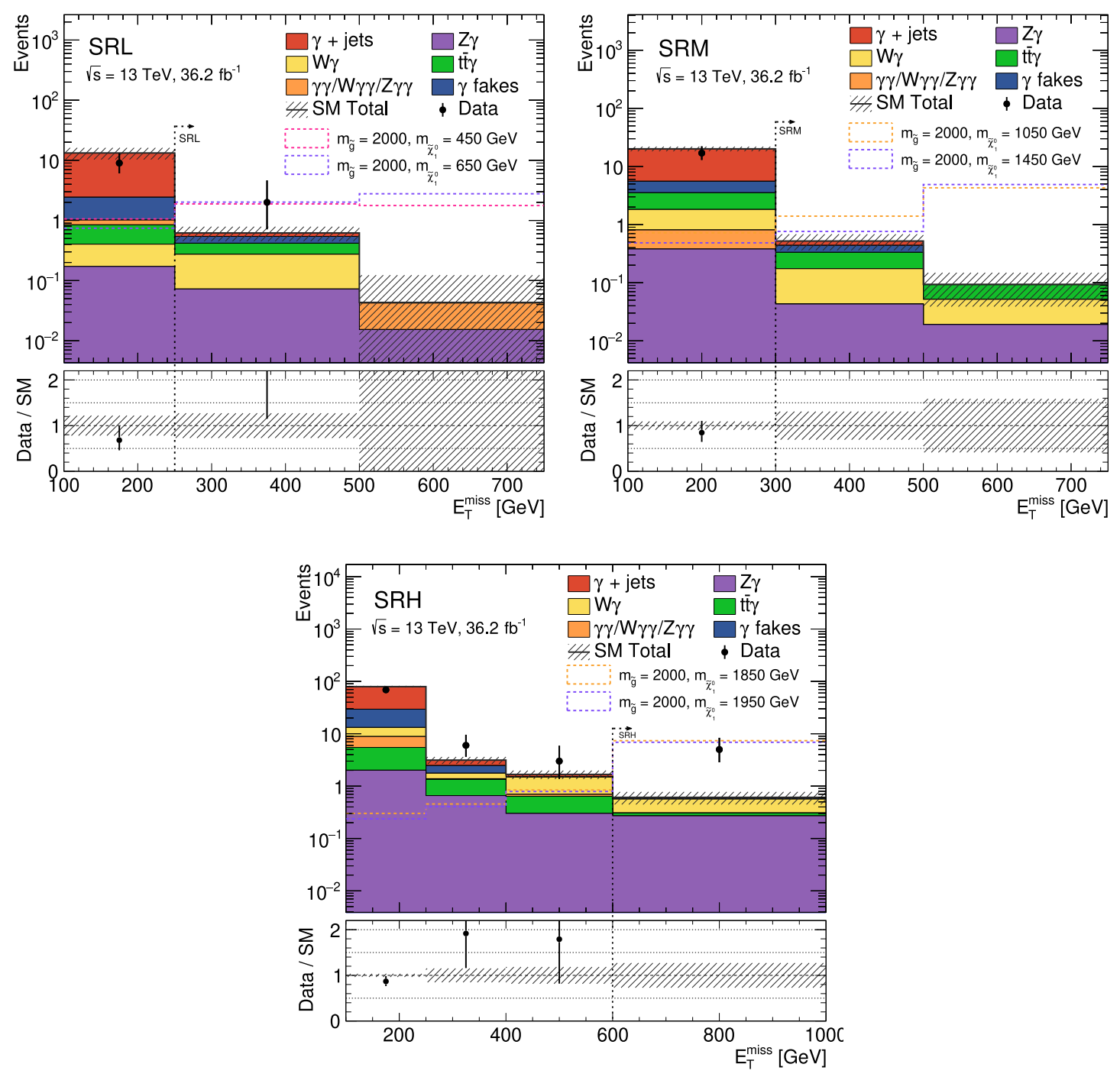

Figura 6.16: Distribuciones observadas (puntos con barras de incerteza) y fondo esperado (histogramas sólidos) $E_{\mathrm{T}}^{\text {miss }}$ en las regiones de señal SRL, SRM y SRH, luego del ajuste de solo-fondo. Las distribuciones para dos modelos de señal, con los valores especificados para las masas de gluinos y neutralinos se agregan para comparación. Las incertezas en los fondos del SM son sólo estadísticas.

estadística de una señal, se considera que los datos observados con $36.2 \mathrm{fb}^{-1}$ son aún compatibles con el fondo esperado y se procede en la sección siguiente a la obtención de los límites superiores a $95 \%$ CL en la producción de nueva física compatible con la búsqueda presentada en esta tesis.

\subsection{Límites independientes del modelo}

Basado en el número de eventos observados en las SR y los eventos de fondo esperados mostrados en la Tabla 6.7, se establecen límites superiores, con nivel de confianza (CL) de $95 \%$ para cada SR, en el número de eventos de cualquier escenario de física más allá del SM $\left(S_{\mathrm{obs}}^{95}\right)$, compatible con la búsqueda realizada en esta tesis. 


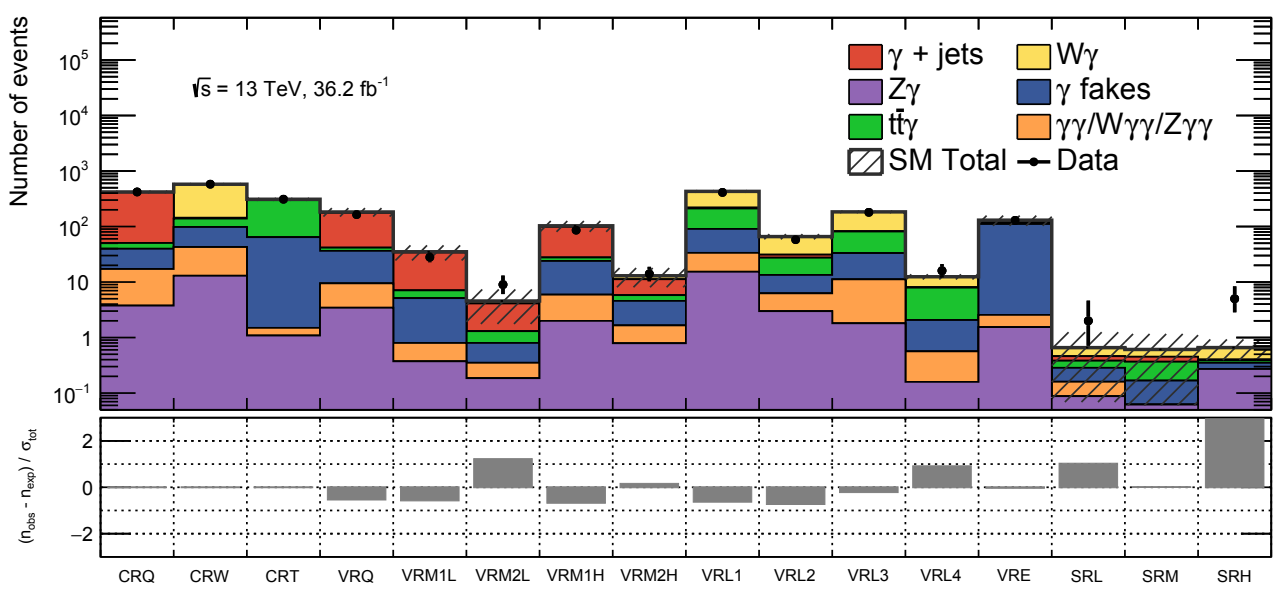

Figura 6.17: El número de eventos observados y esperados en las regiones de control, validación y señal (blinded), para el conjunto de datos de $2015+2016$, correspondientes a $36.2 \mathrm{fb}^{-1}$. El panel inferior muestra la diferencia, en desviaciones estándar, entre el número de eventos observados y esperados (pulls). El pull se calcula utilizando las incertezas sistemáticas y estadísticas en la predicción del fondo $\left(\sigma_{\text {tot }}\right)$.

Tabla 6.8: Las primeras dos columnas muestran el número de eventos observados y esperados. Luego, el límite superior al 95\% CL en la sección eficaz visible $\left(\langle\epsilon \sigma\rangle_{\text {obs }}^{95}\right)$ y en el número de eventos de señal $\left(S_{\mathrm{obs}}^{95}\right)$, y en el número de eventos de señal dado el número esperado (y apartamientos de $\pm 1 \sigma$ en lo esperado) de eventos de fondo $\left(S_{\exp }^{95}\right)$. Finalmente se muestra el $p$-value de descubrimiento $(p(s=0))$.

\begin{tabular}{lcccccc}
\hline Región de Señal & $N_{\text {obs }}$ & $N_{\exp }$ & $\langle\epsilon \sigma\rangle_{\text {obs }}^{95}[\mathrm{fb}]$ & $S_{\text {obs }}^{95}$ & $S_{\exp }^{95}$ & $p(s=0)$ \\
\hline SRL & 2 & $0.66 \pm 0.59$ & 0.18 & 6.4 & $4.9_{-0.4}^{+2.2}$ & 0.13 \\
SRM & 0 & $0.61 \pm 0.55$ & 0.02 & 0.7 & $3.0_{-0.1}^{+1.2}$ & 0.50 \\
SRH & 5 & $0.66 \pm 0.27$ & 0.27 & 9.6 & $4.2_{-0.3}^{+0.7}$ & 0.001 \\
\hline
\end{tabular}

Esto se realiza utilizando el profile likelihood ratio [169] y las prescripciones CL $_{s}$ [170]. Como se mencionó en la Sección 5.5, este ajuste tiene en cuenta solo el fondo, para ser independiente del modelo. En este caso, para cada SR, se realiza un muestreo sobre el parámetro de interés, que es el número de eventos de señal para un dado modelo. El límite superior en el número de eventos de nueva física se encuentra para el valor en el cual el $\mathrm{CL}_{s}$ cae debajo del $5 \%$.

Dichos límites se presentan en la Tabla 6.8, junto con los límites en la sección eficaz visible $\left(\langle\epsilon \sigma\rangle_{\text {obs }}^{95}\right)$, que se deriva simplemente de dividir el límite en el número de eventos por la luminosidad integrada. Asimismo, se agregan al final de la misma tabla, los límites en el número de eventos de señal, dado el número esperado de eventos de fondo $\left(S_{\exp }^{95}\right)$, junto con el $p$-value de descubrimiento $(p(s=0))$.

Se tiene que el límite en el número de eventos de nueva física para SRL, SRM y SRH es 6.4, 0.7 y 9.6, respectivamente. De lo anterior y el valor de la luminosidad utilizada en este análisis $\left(36.2 f b^{-1}\right)$, los límites en la sección eficaz visible encontrados son $0.18 f b^{-1}$ para SRL, $0.02 f b^{-1}$ para SRM y $0.27 f b^{-1}$ para SRH. 


\subsection{Límites dependientes del modelo}

Los límites al modelo de SUSY considerado en esta tesis se establecen al $95 \%$ de CL usando la receta de $\mathrm{CL}_{s}$ y se hacen por el ajuste de exclusión. En este caso, en el ajuste simultáneo del likelihood se tienen en cuenta tanto el fondo como la señal. Se tiene entonces que la intensidad de señal es $\mu_{\text {sig }} \neq 0$, se hace un ajuste independiente por cada SR, y se repite para cada punto del espacio observable de señal. Teniendo en cuenta las incertezas teóricas de la señal, el límite de exclusión observado se calcula con el número de eventos de señal correspondiente al valor nominal de la sección eficaz del modelo de SUSY $\pm 1 \sigma$.

La definición de cada una de las SR se realiza para aumentar la sensibilidad en distintas partes de la grid. Como se describe en la Sección 5.3, SRL se optimiza para cubrir la región a baja masa del neutralino, mientras que SRH lo hace en los escenarios comprimidos con valores de masas similares entre el gluino y el neutralino. Por su parte, SRM cubre la región intermedia de masas. Debido a esto, se obtienen los límites para cada una de estas SR por separado, y se las combina para obtener un único límite combinado.

La Figura 6.18 muestra los límites esperados y observados combinados, usando la región de señal con la mejor sensibilidad esperada en cada punto, obtenidos usando pseudo-experimentos ( 20000 toys). La línea negra punteada muestra el límite esperado y las bandas amarillas indican la desviación del mismo en $\pm 1 \sigma$ (considerando incertezas experimentales y teóricas). La línea sólida roja muestra el límite observado y las punteadas del mismo color, representan dicho límite para las variaciones de la sección eficaz de la señal (incerteza teórica de la muestra se señal).

Considerando el límite observado, se excluye a $95 \%$ CL la producción de gluinos con masas de hasta $\sim 2100 \mathrm{GeV}$, para masas intermedias de neutralinos $\sim 1000 \mathrm{GeV}$. Debido a la menor aceptancia del análisis en las regiones con baja masa del neutralino, la exclusión alcanzada se reduce hasta masas de gluinos de $1700 \mathrm{GeV}$. 


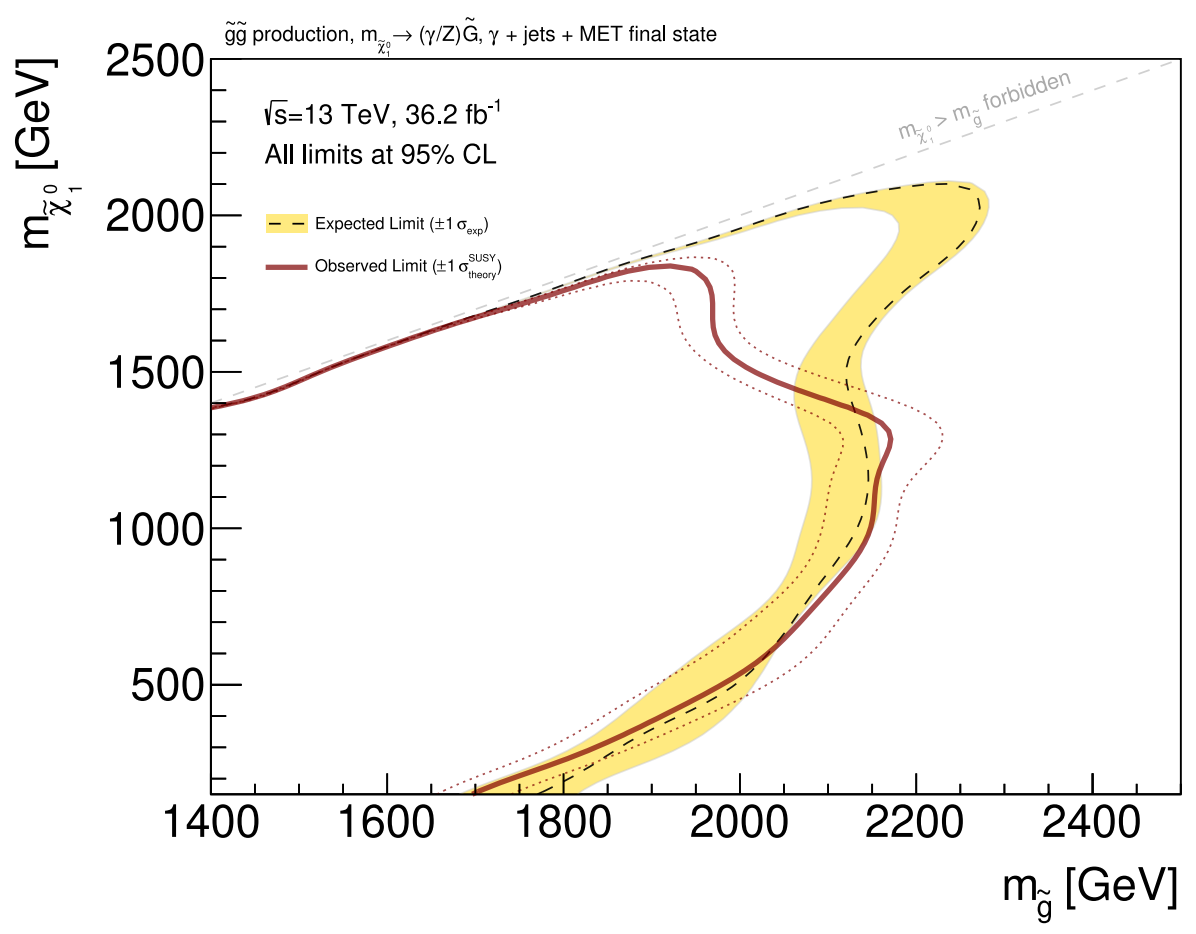

Figura 6.18: Límites de exclusión al 95\% CL observados y esperados, usando la región de señal con la mejor sensibilidad esperada en cada punto, para el conjunto de datos de $2015+2016$ correspondientes a una luminosidad integrada de $36.2 \mathrm{fb}^{-1}$. La línea negra punteada muestra el límite esperado y las bandas amarillas indican la desviación $\pm 1 \sigma$ (considerando incertezas experimentales y teóricas). La línea sólida roja muestra el límite observado y las punteadas representan el mismo límite para las variaciones de la sección eficaz de la señal (incerteza teórica de la muestra se señal). 


\section{Capítulo 7}

\section{Nuevas búsquedas en modelos extendidos}

El principal objetivo científico que motivó la construcción del LHC y sus detectores multipropósito, fue la búsqueda del bosón de Higgs (cumplido en 2012 con el descubrimiento) y la búsqueda de física más allá del SM. Si bien estas búsquedas de nueva física no estuvieron nunca restringidas a teorías específicas, desde el comienzo de la era del LHC tuvieron una muy fuerte motivación en modelos de SUSY como se puede ver en el reporte de ATLAS de 1999 [171]. En esta tesis no sólo se discutieron las bases y motivaciones teóricas detrás de SUSY (Capítulo 1), sino que además se presentó un análisis completo de una búsqueda motivada en modelos GMSB-GGM (Capítulos 5 y 6). Para una visión más general, los límites a las masas de las spartículas representativas de los distintos modelos de SUSY analizados en ATLAS se muestran en la Figura 7.1. De esta figura no sólo se puede ver el enorme esfuerzo puesto por la colaboración para cubrir, con modelos simplificados y estados finales accesibles, una enorme porción del espacio de parámetros, sino que también queda explícito que la idea de SUSY natural (aquella que resuelve los problemas de naturalidad del SM), con masas de partículas en el orden de $2 \mathrm{TeV}$, se pone en duda en base a los resultados de modelos simplificados hasta ahora estudiados. Al mismo tiempo, una particularidad que tienen la gran mayoría de los modelos estudiados es que conservan la paridad $\mathrm{R}$ y en general presentan un alto requerimiento en $E_{\mathrm{T}}^{\text {miss }}$. Esto significa que, a pesar de la amplia cobertura que se ve en la figura anterior, existen todavía huecos en el espacio de parámetros, donde es posible buscar supersimetría.

A baja luminosidad y en los primeros años de búsqueda en el LHC, modelos simplificados en términos de número de parámetros y sensibles en términos de sus secciones eficaces y BRs fueron seleccionados para motivar las distintas búsquedas. Actualmente, se están comenzando a realizar esfuerzos que involucren modelos más complejos pero con sensibilidades menores, que podrán ser sólo alcanzados a mayores luminosidades. En esta dirección se presenta a continuación, un caso de estudio para una búsqueda de SUSY en ATLAS, motivada en modelos de RPC Stealth SUSY (véase Sección 1.5.2), con estados finales formados por múltiples jets, fotones y bajo momento transverso faltante. 


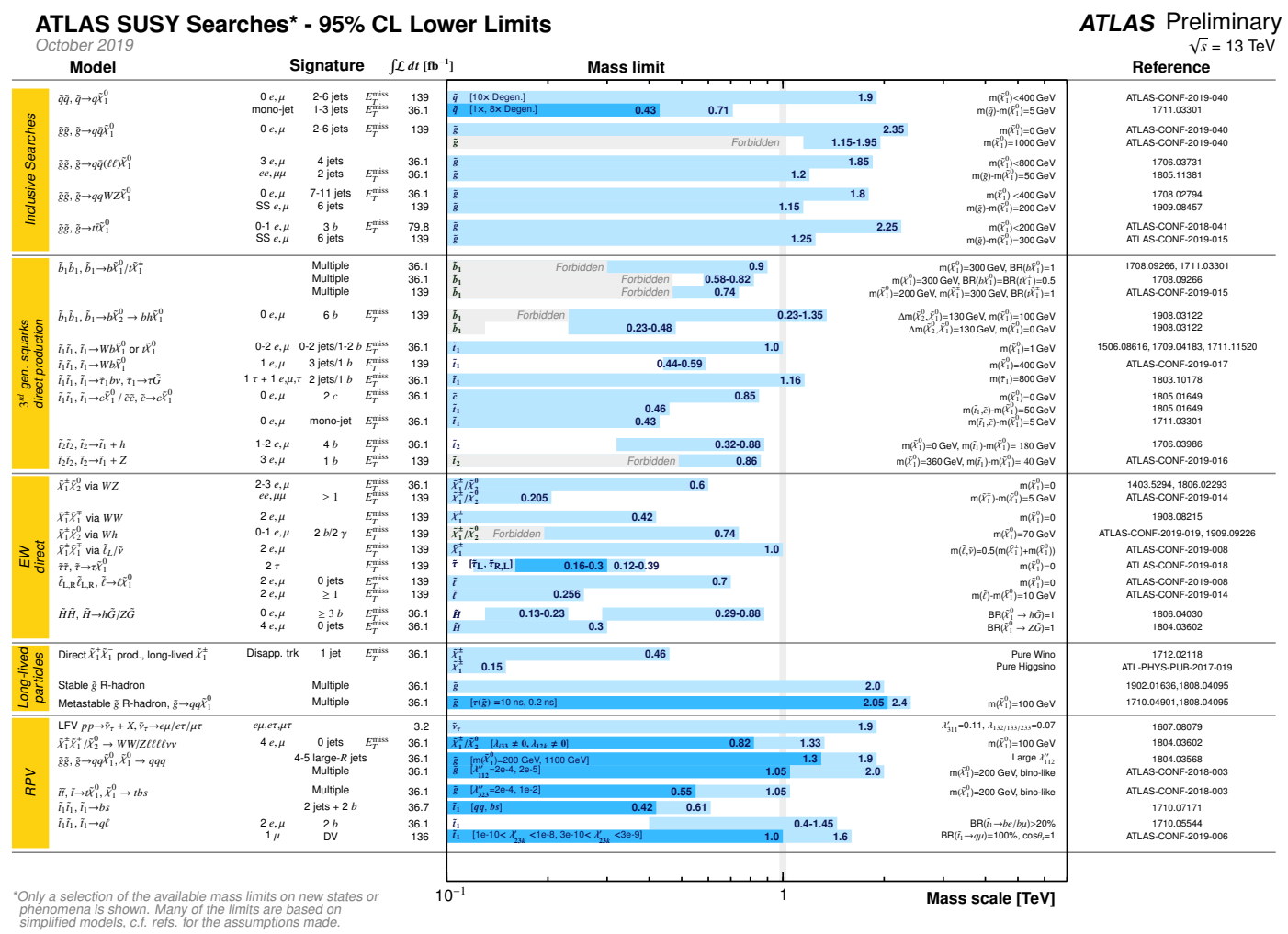

Figura 7.1: Alcance de la masa en las búsquedas de SUSY en ATLAS. Se muestra una selección representativa de los resultados de las búsqueda disponibles. Los resultados se mencionan para la sección transversal nominal tanto en una región de alcance de masa casi máxima como también en un escenario alternativo demostrativo. Algunos límites dependen de supuestos adicionales sobre la masa de los estados intermedios, tal como se describe en las referencias proporcionadas en el gráfico. En algunos casos estas dependencias adicionales se indican mediante bandas más oscuras que muestran diferentes parámetros del modelo.

\section{1. $\quad$ Modelos y simulaciones}

Los diagramas analizados en este estudio preliminar se muestran en la Figura 7.2. Son modelos donde el proceso se inicia con una producción de pares de squarks o gluinos (estos últimos decaen a un squark y a partir de allí ambos modelos tienen la misma cadena). Los squarks decaen a un jet y al $\widetilde{\chi}_{1}^{0}$ que, en este caso, se considera bino puro decayendo con un BR de $100 \%$ a un fotón y al singlino $(\widetilde{S})$, que a su vez decae al singlete $(S)$ y a la LSP de la teoría, el gravitino $(\widetilde{G})$. El singlete decae finalmente a dos jets, y debido a la casi degeneración de masas entre las partículas stealth, el momento transverso del $\widetilde{G}$ será bajo, dando el estado final deseado con fotones, jets y bajo $E_{\mathrm{T}}^{\text {miss }}[48,49]$.

Para realizar este estudio, se generan muestras MC de los modelos de señal antes mencionados para el conjunto de masas especificadas en la Tabla 7.1. La generación se realiza con el software MadGraph5+Pythia8, utilizando para el cálculo de las secciones eficaces el programa NNLL-fast [129, 130, 131, 132, 133]. La producción se realiza solo a nivel generador (MC TRUTH) para este primer estudio exploratorio sobre la sensibilidad y posibilidad de esta búsqueda. Para cada punto simulado (y para cada conjunto de masas) se generan cinco mil eventos. 

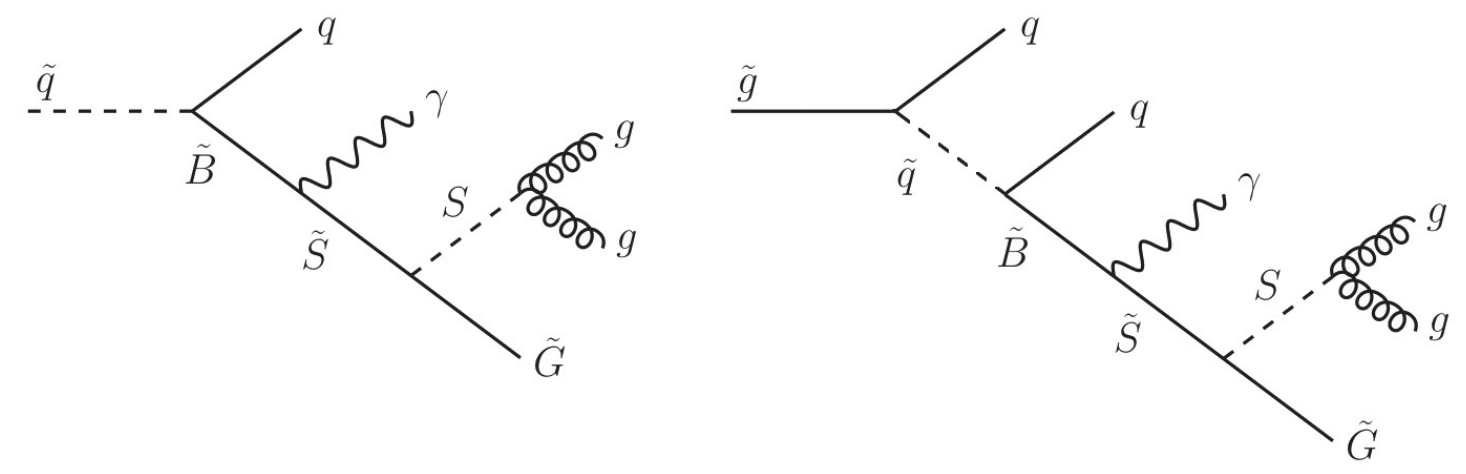

Figura 7.2: Decaimientos de Stealth SUSY, a partir de la producción de pares de squarks o gluinos, con un neutralino bino puro que decae a un fotón y singlino.

Tabla 7.1: Conjunto de masas para las distintas partículas involucradas en los decaimientos analizados, necesarias para generar las muestras MC de señal.

\begin{tabular}{|c|c|c|c|c|c|}
\hline Producción & $\widetilde{g}$ & $\widetilde{q}$ & $\widetilde{\chi}_{1}^{0}$ & $\widetilde{S}$ & $S$ \\
\hline \multirow{3}{*}{$\widetilde{g}-\widetilde{g}$} & \multirow{3}{*}{1800} & \multirow{2}{*}{1400} & 1200 & \multirow{2}{*}{1000} & \multirow{2}{*}{900} \\
\hline & & & 1100 & & \\
\hline & & 1200 & $\begin{array}{l}000 \\
600\end{array}$ & 600 & 540 \\
\hline \multirow{5}{*}{$\widetilde{q}-\widetilde{q}$} & \multirow{5}{*}{ 一 } & \multirow{5}{*}{1200} & 1100 & م०ח & ח7ר \\
\hline & & & 800 & 300 & 210 \\
\hline & & & 600 & & \\
\hline & & & 400 & 100 & 90 \\
\hline & & & 200 & & \\
\hline
\end{tabular}

Con el fin de simplificar la discusión, en lo que resta del capítulo se muestran los resultados solo para la producción de squarks. Este proceso de producción de Stealth SUSY resulta con una sección eficaz mayor (que la producción de gluinos), favoreciendo la presencia de señal, pero al mismo tiempo, presenta baja multiplicidad hadrónica lo que dificulta el rechazo del fondo contaminante.

\section{2. $\quad$ Selección de objetos y distribuciones}

Los requerimientos de selección utilizados para los distintos objetos físicos se listan a continuación:

- EMTopo anti- $k_{T}(R=0.4)$ Jets con $p_{\mathrm{T}}>50 \mathrm{GeV}$ y $|\eta|<2.4$.

- Fotones tight y aislados (FixedCutTight) con $p_{\mathrm{T}}>50 \mathrm{GeV}$ y $|\eta|<2.37$.

- Electrones tight y aislados (FCLoose) con $p_{\mathrm{T}}>20 \mathrm{GeV}$ y $|\eta|<2.47$.

- Muones medium y aislados (FCLoose) con $p_{\mathrm{T}}>20 \mathrm{GeV}$ y $|\eta|<2.5$.

En esta primera etapa exploratoria se minimiza el número de cortes de selección requeridos para que la búsqueda propuesta sea lo más inclusiva posible. Se requiere 

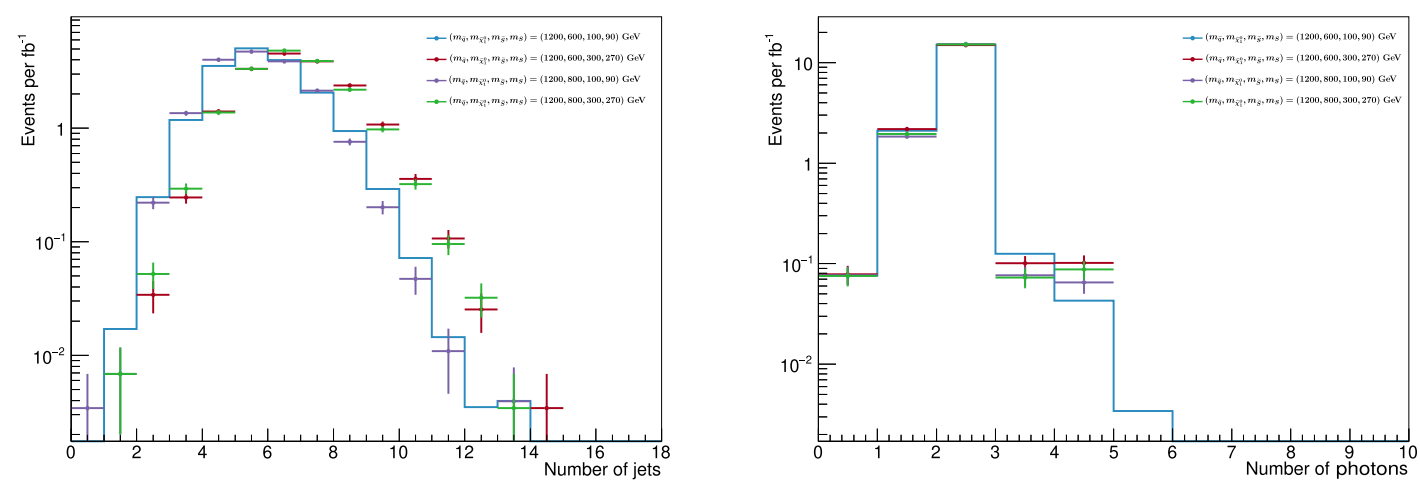

Figura 7.3: Distribuciones para la multiplicidad de jets (izquierda) y de fotones (derecha), para los cuatro puntos de referencia.

un veto de leptones, debido a la ausencia de éstos en el estado final. El solapamiento entre objetos se realiza siguiendo las mismas recomendaciones aplicadas en la Sección 5.1.5. El momento transverso faltante se calcula de igual manera que en la Sección 5.1 .6

Los puntos de señal generados se eligen para cubrir las distintas regiones cinemáticas características de estos procesos, donde se estima pueda tener sensibilidad una búsqueda en el LHC. En particular, los más representativos que se eligen como referencia para presentar y comparar en lo que sigue, son aquellos con $m_{\widetilde{q}}=1200 \mathrm{GeV}$, $m_{\widetilde{\chi}_{1}^{0}}=600$ u $800 \mathrm{GeV}, m_{\widetilde{S}}=300$ o $100 \mathrm{GeV}$ y $m_{S}=270$ o $90 \mathrm{GeV}$, respectivamente.

Todas las distribuciones que se presentan a continuación están normalizadas a la cantidad de eventos por $f b^{-1}$. En primer lugar, las distribuciones para la multiplicidad de jets y fotones de estos cuatro puntos de referencia se muestran en la Figura 7.3. Es importante notar aquí que los máximos de estas distribuciones coinciden con los valores esperados para este modelo, siendo $N_{\text {jets }}=6$ y $N_{\gamma}=2$.

Las distribuciones de $H_{\mathrm{T}}$ y $\mathcal{S}\left(E_{\mathrm{T}}^{\text {miss }}\right)$ se muestran en la Figura 7.4. En este caso se define $H_{\mathrm{T}}$ como:

$$
H_{\mathrm{T}}=\sum_{j e t s} p_{\mathrm{T}}^{\text {jets }}+p_{\mathrm{T}}^{\text {leading } \gamma}+p_{\mathrm{T}}^{\text {subleading } \gamma}
$$

donde, a diferencia de la definición que se utiliza en la búsqueda GGM de SUSY anterior, ahora se considera también el $p_{\mathrm{T}}$ del segundo fotón en el evento. A su vez, la significancia de $E_{\mathrm{T}}^{\text {miss }} \mathcal{S}\left(E_{\mathrm{T}}^{\text {miss }}\right)$, cuantifica el grado en que el $E_{\mathrm{T}}^{\text {miss }}$ medido es incompatible con su origen puramente de fluctuaciones y mediciones erróneas. Un valor grande de esta variable (relativo al valor de $E_{\mathrm{T}}^{\text {miss }}$ ) indica que $E_{\mathrm{T}}^{\text {miss }}$ se origina genuinamente a partir de partículas no interactuantes. En el presente estudio se utiliza la siguiente definición de esta variable [172]:

$$
\mathcal{S}\left(E_{\mathrm{T}}^{\text {miss }}\right)=\frac{E_{\mathrm{T}}^{\text {miss }}}{\sqrt{H_{\mathrm{T}}}}
$$

De estas distribuciones se corrobora la gran escala de energía de los eventos de señal (con un máximo en $H_{\mathrm{T}} \sim 2 \mathrm{TeV}$ ) y, debido al bajo $E_{\mathrm{T}}^{\text {miss }}$, se obtienen 

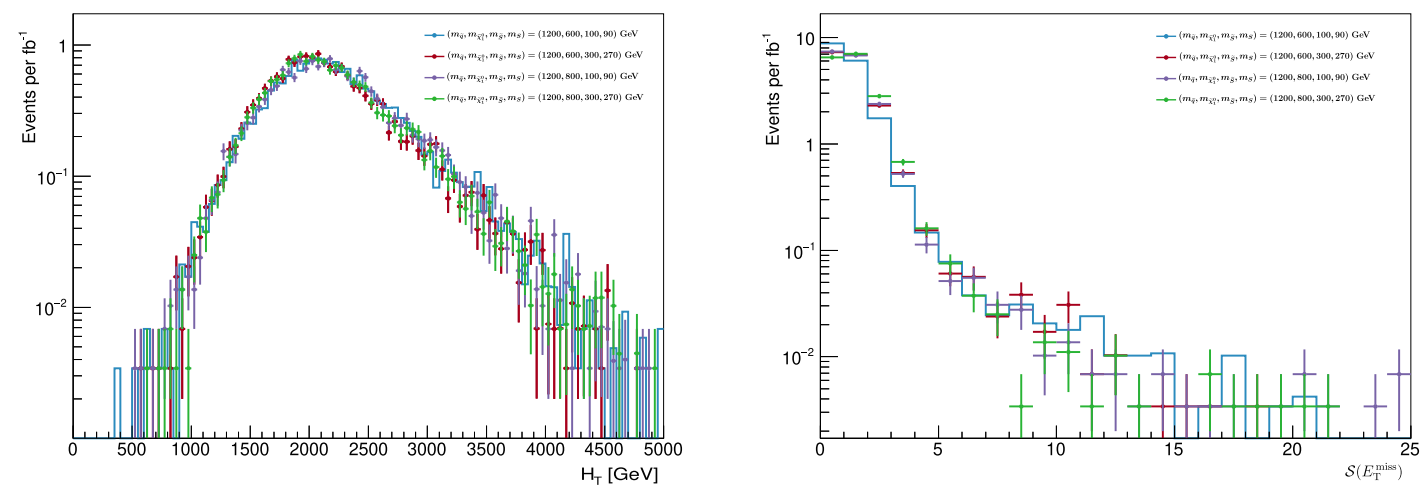

Figura 7.4: Distribuciones para las variables $H_{\mathrm{T}}$ (teniendo en cuenta los dos fotones más energéticos) y $\mathcal{S}\left(E_{\mathrm{T}}^{\mathrm{miss}}\right)$.
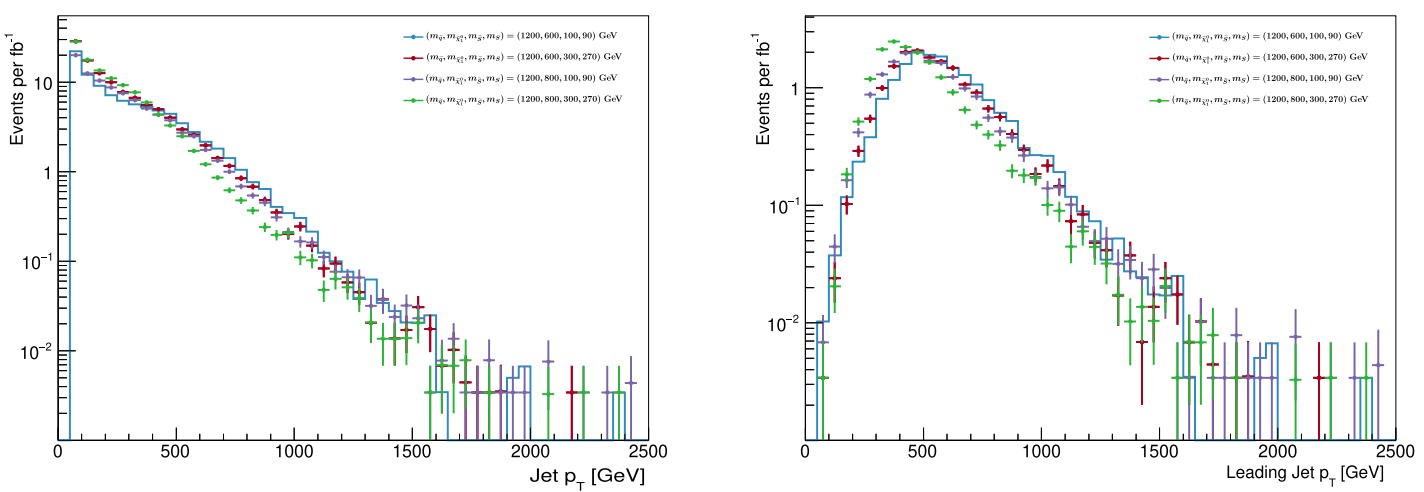

Figura 7.5: Distribuciones de $p_{\mathrm{T}}$ para todos los jets del evento (izquierda) y para el jet con más alto momento (derecha).

bajos valores de $\mathcal{S}\left(E_{\mathrm{T}}^{\text {miss }}\right)$ comparables a los esperados para eventos de fondo QCD del SM. Esta última característica permite suponer que el poder discriminatorio de esta variable no será significativo, a diferencia de lo que sucede en búsquedas con multijets y alto $E_{\mathrm{T}}^{\text {miss }}$ [113].

Por último, se presentan las distribuciones de $p_{\mathrm{T}}$ para todos los jets en el evento y para el jet con el momento más grande entre ellos (leading jet) en la Figura 7.5, y de manera similar para los fotones en la Figura 7.6, donde se agrega además la distribución para el fotón sub-leading. Se espera que todas estas variables sean las de mayor poder de discriminación entre la señal y el fondo SM.

\subsection{Estudios preliminares de factibilidad}

Como primer estudio preliminar para evaluar la factibilidad de una posible búsqueda, se comparan las distribuciones y el correspondiente número de eventos para el modelo y para una fracción mínima de los datos disponibles. Dicha fracción se utiliza como una primera aproximación del fondo del SM. En este caso se utilizan datos del 2015 que, al ser solo una preselección de base de una muestra pequeña, se suponen dominados por eventos de fondo y son entonces utilizados como tales en la 

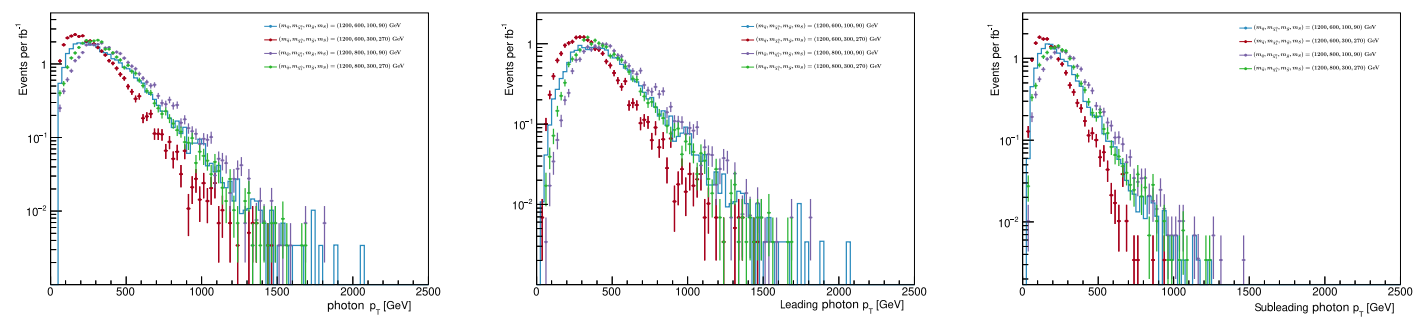

Figura 7.6: Distribuciones de $p_{\mathrm{T}}$ para todos los fotones del evento (izquierda), para el fotón leading (centro), y para el subleading (derecha).

comparación siguiente.

La Tabla 7.2 muestra una comparación de eventos entre dos modelos de Stealth SUSY y datos $2015\left(3.2 \mathrm{fb}^{-1}\right)$, para los diferentes cortes que seleccionan el estado final, aplicando entonces una selección de seis jets con $p_{\mathrm{T}}$ mayor a $50 \mathrm{GeV}$, al menos dos fotones aislados de $50 \mathrm{GeV}$ y un veto en la presencia de leptones en el estado final. Ambos modelos tienen $m_{\widetilde{q}}=1200 \mathrm{GeV}, m_{\widetilde{\chi}_{1}^{0}}=600 \mathrm{GeV}$, y las masas de los singlinos y singletes: $(\widetilde{S}, S)=(300,270)$ o $(\widetilde{S}, S)=(100,90)$. El número de eventos MC se escala a la luminosidad de 2015 para realizar la comparación.

Tabla 7.2: Comparación de eventos entre dos modelos de Stealth SUSY y datos 2015, para los diferentes cortes que seleccionan el estado final. Sin ninguna otra optimización.

\begin{tabular}{|c|c|c|c|}
\hline Cortes & $(100,90)$ & $\begin{array}{l}S) \\
(\mathbf{3 0 0 , 2 7 0 )}\end{array}$ & Data15 \\
\hline Todos & \multicolumn{2}{|c|}{55.7} & \\
\hline $0 \mathrm{~L}$ & 48.5 & 47.9 & \\
\hline $6 \mathrm{j} 50$ & 21.5 & 35.5 & $1.23 \mathrm{E}+06$ \\
\hline 1ph50 & 21.4 & 35.4 & 7700 \\
\hline 2ph50 & 18.8 & 31.5 & 70 \\
\hline
\end{tabular}

Si se consideran sistemáticos del $30 \%$ y un número de eventos de fondo igual a los obtenidos en datos, se puede calcular la significancia (Ec. 5.2), resultando en valores cercanos a la unidad para ambos modelos.

Esta relación preliminar entre señal y fondo, obtenida a partir de una selección de base y previa a un proceso de optimización, permite considerar factible la presente búsqueda, sentando las bases para una futura investigación dentro del experimento ATLAS, motivada por modelos de Stealth SUSY.

\subsubsection{Impacto de selección de eventos por un trigger combi- nado}

Con el objetivo de optimizar las búsquedas de Stealth SUSY tomando como base un estado final con alto número de jets y al menos un fotón, se propuso la implementación de un nuevo trigger combinando los distintos objetos mencionados. Como se muestra en la Sección 4.5, este nuevo trigger que combina el requerimiento de un fotón loose con $p_{\mathrm{T}}>45 \mathrm{GeV}$ y seis jets con igual requerimiento en $p_{\mathrm{T}}, \mathrm{y}$ 


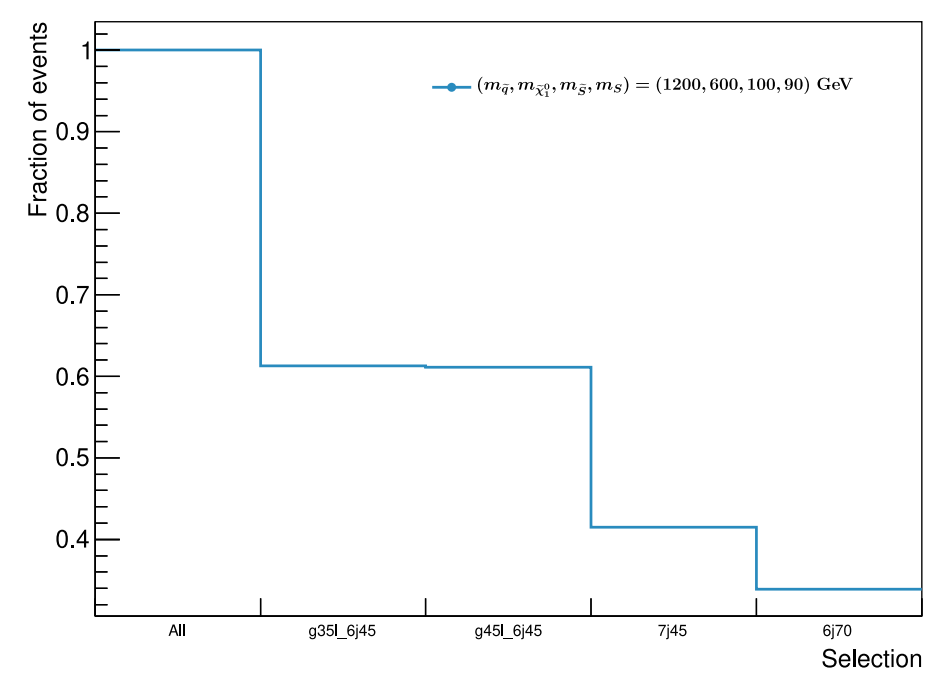

Figura 7.7: Fracción de eventos disponibles luego del requerimiento equivalente de distintos triggers según se muestra en su nomenclatura. La selección de cada bin es independiente de los otros, cada requerimiento es siempre con respecto al total de la muestra.

dentro del rango $0<|\eta|<2.4$, HLT_g45_loose_6j45_0eta240, se incluyó para la toma de datos de 2018 .

Para la selección de los eventos, dos de los triggers de multijets sin pre-escaleo disponibles en el menú que podrían utilizarse son HLT_6j70_0eta240 y HLT_7j45_0eta240, que requieren 6 o 7 jets con un $p_{\mathrm{T}}$ de al menos 70 o $45 \mathrm{GeV}$, respectivamente. Debido a la presencia de un fotón en el estado final para el modelo de Stealth SUSY analizado, la inclusión de este nuevo trigger combinado permite reducir los requerimientos finales de las posibles regiones de señal desde el trigger.

La ganancia en señal que ofrece este nuevo trigger combinado con respecto a los otros dos triggers disponibles en el menú, se estudia con una muestra MC de 5 mil

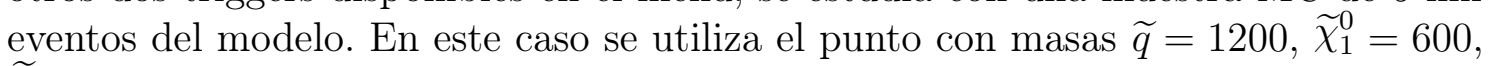
$\widetilde{S}=100$ y $S=90$, y en la Figura 7.7 se muestra la fracción de eventos disponible luego del requerimiento de alguno de los triggers. Notar aquí que la selección de cada bin es independiente de los otros. Cada requerimiento es siempre con respecto al total de la muestra.

De la figura anterior se desprende que la fracción de eventos que se obtiene después de requerir 7 jets con $p_{\mathrm{T}}>45 \mathrm{GeV}$ o 6 jets con $p_{\mathrm{T}}>70 \mathrm{GeV}$, es entre un 20 y un $30 \%$ menor que aquella lograda por el trigger combinado propuesto. Un estudio similar se hizo para un modelo de GMSB SUSY, conocido como GTTP, donde el gluino decae a quarks tops y al neutralino más liviano que a su vez decae a un fotón y gravitino que, debido al estado final con una alta multiplicidad de jets y al menos un fotón, lo vuelve un posible usuario de este trigger, y del cual obtendría una ganancia en señal de $\sim 40 \%$. 


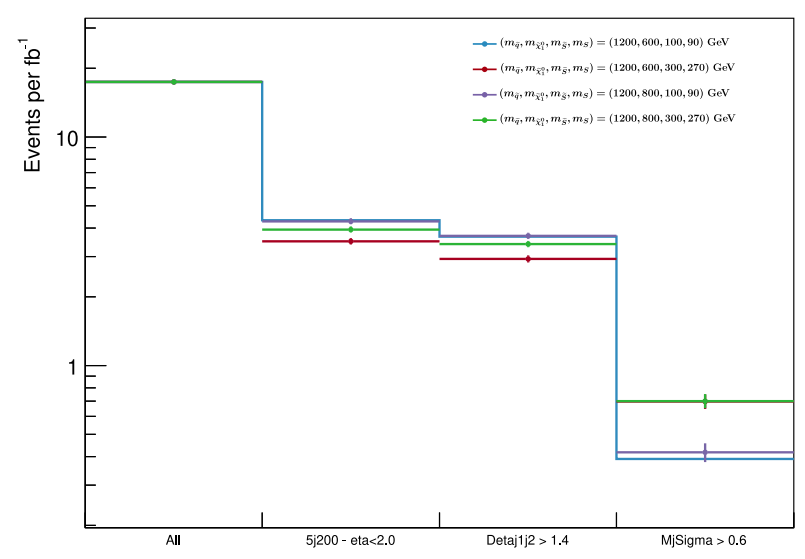

Figura 7.8: Cutflow para la región con 5 jets del análisis $R P V$ multijets [173], para los cuatro puntos de referencia de Stealth SUSY.

\subsubsection{Reinterpretación simple de Nueva Física con Stealth SUSY}

Dentro de la colaboración ATLAS están documentadas todas las búsquedas de SUSY en un paquete de software llamado SimpleAnalysis, que permite evaluar un dado modelo teórico en dichas búsquedas. Esta herramienta provee, de manera simple y en un formato sistematizado, la selección de eventos para las distintas regiones de señal y control de las distintas búsquedas. En base a esta información, es posible determinar la aceptancia de los distintos análisis ya realizados sobre un nuevo modelo a estudiar. Una vez determinadas las búsquedas con mayor aceptancia y con la información de los fondos esperados, se puede entonces determinar la exclusión (o no) del nuevo modelo propuesto.

Se realiza dicho estudio con una muestra de MC de señal con $\widetilde{q}=1200, \widetilde{\chi}_{1}^{0}=600$, $\widetilde{S}=300$ y $S=270$, encontrándose que todas las búsquedas disponibles presentan aceptancias con valores nulos (o compatibles con éstos). Estos resultados eran de esperar debido al alto $E_{\mathrm{T}}^{\text {miss }}$ que requieren la mayoría de las regiones de señal de las búsquedas de SUSY en ATLAS.

Un caso particular que no tiene requerimientos en $E_{\mathrm{T}}^{\text {miss }}$, es el análisis $R P V$ multijets [173]. Este análisis considera jets con un $\mathrm{R}$ grande $(R=1.0)$, y sus requerimientos principales son cuatro (o cinco) jets con $p_{\mathrm{T}}>200 \mathrm{GeV}, \Delta R>1.4$ entre el leading y el subleading jet, y $M_{J}^{\Sigma}>0.6$. La variable $M_{J}^{\Sigma}$ se define como la suma de las masas de todos los jets $(R=1.0)$ con $p_{\mathrm{T}}>100 \mathrm{GeV}$ y $|\eta|<1.5$. Los resultados de aplicar los cortes de selección (cutflow) para los cuatro puntos de referencia en la región con cinco jets se muestran en la Figura 7.8. Se observa que la variable $M_{J}^{\Sigma}$, con alto poder discriminatorio para dicho análisis, no es apropiada para su uso en Stealth SUSY.

Una comparación entre eventos del MC de señal de Stealth SUSY aplicando la selección de las SR del análisis de RPV, y los datos compatibles con el fondo del SM según el análisis realizado con $14.82 \mathrm{fb}^{-1}$, se muestra en la Tabla 7.3. Las columnas $4 \mathrm{j}$ y $5 \mathrm{j}$ refieren a si el análisis requiere 4 o 5 jets en sus regiones de señal. 
Tabla 7.3: Comparación entre eventos del MC de señal de Stealth SUSY (para los distintos cortes de las SR de RPV) y los observados en datos en el análisis con $14.82 \mathrm{fb}^{-1}$.

\begin{tabular}{l|l||cc} 
& Selección & $4 \mathrm{j}$ & $5 \mathrm{j}$ \\
\hline \hline MC de señal & $4 \mathrm{j} 200, \eta<2.0$ & 157.9 & - \\
& $5 \mathrm{j} 200, \eta<2.0$ & - & 51.6 \\
& $\eta_{12}<1.4$ & 138.9 & 43.4 \\
& $M_{j}^{\Sigma}>0.8$ & 15.7 & - \\
& $M_{j}^{\Sigma}>0.6$ & - & 10.34 \\
\hline \hline Datos & SR & 122 & 64
\end{tabular}

En base a los resultados esperados se puede estimar la significancia de la búsqueda, obteniéndose valores de ésta entre 0.2 y 0.3 según el número de jets seleccionados. Siendo éste el análisis más favorable, queda determinado que el modelo objeto de nuestro estudio Stealth SUSY, no ha sido excluido por las búsquedas ya realizadas dentro de la colaboración.

Surge entonces la necesidad de iniciar un nuevo análisis que lleve adelante estos estudios que permitirán cubrir aún más el espacio de parámetros de las búsquedas de SUSY en ATLAS. 


\section{Capítulo 8}

\section{Conclusión}

El éxito de la operación del LHC ha quedado plasmado en los casi $140 \mathrm{fb}^{-1}$ de datos de colisiones colectados por el detector ATLAS a energía de centro de masa de $13 \mathrm{TeV}$. Esta gran riqueza de datos ha permitido avances en medidas de precisión del Modelo Estándar y búsquedas de nueva física más allá de nuestra comprensión actual, como la que se presenta en esta tesis. Desde el comienzo del programa de búsqueda de supersimetría en los experimentos del LHC, se consideró una vasta cantidad de escenarios generales, sin embargo al presente no se ha observado evidencia de nuevas partículas supersimétricas lo que motiva un esfuerzo por mejorar el poder de exclusión. Si bien se planea aumentar considerablemente los datos colectados con ATLAS en el futuro Run-3, no se espera un enorme aumento en la sensibilidad de algunos modelos específicos, dada la abundante cantidad de datos con los que se cuenta al presente. Por esta razón, ha sido necesario focalizarse en desarrollos desde el sistema de trigger, incluyendo la reconstrucción de objetos físicos y su posterior selección, y en optimizaciones de los métodos de análisis, a fin de poder mejorar drásticamente la sensibilidad de la búsqueda respecto de las investigaciones previas en Run-1 aprovechando el conjunto completo de datos de Run-2, todos aspectos desarrollados e incluidos en esta tesis.

Dado que la búsqueda de nueva física aquí presentada ha sido motivada por modelos de SUSY que predicen estados finales con fotones de alto momento transverso, jets y energía transversa perdida, una parte de esta tesis está abocada al estudio de estos objetos físicos tanto como candidatos claves del estado final experimental elegido, como también para la evaluación de la eficiencia del detector en seleccionarlos aportando también a la realización de medidas de precisión y otras búsquedas involucrando estos objetos físicos en sus procesos.

El primer paso de todo análisis queda definido por el sistema de trigger. Esta selección en tiempo real ha sido todo un desafío científico-técnico, ya que se necesitó hacer frente al aumento de cuatro veces la luminosidad máxima del LHC en Run-2 (hasta $2.1 \times 10^{34} \mathrm{~cm}^{-2} \mathrm{~s}^{-1}$ ), y a un aumento similar en el número de interacciones por cruce de haces en comparación con la toma de datos durante el Run-1. A modo de referencia, aplicando los métodos explicados en esta tesis (Capítulo 4) se determinaron las eficiencias para dos de los principales triggers de fotones usados para la toma de datos de la colaboración, que fueron el trigger difotón con umbrales de energía transversal de 25 y $35 \mathrm{GeV}$, y el trigger de un sólo fotón con energía transversal de $140 \mathrm{GeV}$. Para estos se encontraron eficiencias de selección individuales superiores al $96 \%$ para fotones reconstruidos offline con un mínimo de $5 \mathrm{GeV}$ de 
energía por encima del umbral del trigger. Los estudios realizados han sido incluidos en las publicaciones de ATLAS [103, 108].

Es de destacar que estudios con alto número de jets son experimentalmente interesantes y motivan distintos análisis de búsqueda de nueva física. En particular dentro de SUSY se realizó una búsqueda con un estado final que contiene hasta 12 jets y alta energía transversa faltante [113]. En este contexto se implementaron métodos (Capítulo 4) para determinar la performance del sistema de trigger para seleccionar eventos con distinto número de jets, necesarios en dicho análisis, obteniendo eficiencias con valores mayores al $95 \%$ para objetos con $10 \mathrm{GeV}$ por encima del umbral de requerimiento del trigger.

A continuación se presentó el diseño de una búsqueda de nueva física en un estado final de eventos con fotones aislados de alto $p_{\mathrm{T}}$, presencia de jets y alto momento transversal faltante, utilizando $139 \mathrm{fb}^{-1}$ de datos recolectados por el detector ATLAS entre 2015 y 2018. Dicho estado final es favorecido por un modelo GGM-SUSY, con el gravitino como partícula más liviana y un neutralino tipo binohiggsisno como NLSP. En base a este modelo y sus correspondientes simulaciones se diseñaron tres regiones de búsqueda diferentes, SRL, SRM y SRH, en las cuales podrían manifestarse la nueva física llevando a un descubrimiento. La primera región, SRL ha sido optimizada para gluinos de alta masa y neutralinos de baja masa, la $\mathrm{SRH}$ se enfoca en aquellos escenarios en los que las masas del gluino y neutralino son muy parecidas, y la SRM se ocupa de los rangos de masa intermedios. Para determinar si hay un exceso en los datos con respecto a los esperados de procesos del SM, es mandatorio desarrollar estrategias y nuevos métodos para la estimación de los distintos fondos con una alta precisión, en particular frente al escenario de alta estadística que provee el conjunto de datos de Run-2. Estas estrategias fueron detalladas en el presente trabajo, junto con sus respectivas incertezas, tanto experimentales como teóricas. Todo el proceso se demostró finalmente exitoso en base a estudios presentados en distintas regiones de validación especialmente diseñadas para dicha tarea.

En esta tesis se presentan los resultados obtenidos con una muestra parcial de datos del Run-2, colectados en los años 2015 y 2016. Esto permitió mostrar la solidez de la estrategia de búsqueda propuesta y la capacidad, en caso de no encontrar evidencia de nueva física, para realizar el cómputo de los límites en las masas de los neutralinos y gluinos del modelo propuesto así como proveer a la comunidad de los límites en la sección eficaz visible. En cada región de señal se observaron 2, 0, 5 eventos con una predicción esperada para fondo de procesos del SM de $0.66 \pm 0.59$, $0.61 \pm 0.55,0.66 \pm 0.27$ para las regiones $\mathrm{SRL}, \mathrm{SRM}$ y $\mathrm{SRH}$, respectivamente. Estos resultados permiten excluir la producción de gluinos con masas de hasta $1700 \mathrm{y}$ $2100 \mathrm{GeV}$, a $95 \% \mathrm{CL}$, dependiendo de la masa del neutralino, indicando mejoras con respecto a la publicación previa [2]. Dicho aumento en los límites determinados ( $\sim 100 \mathrm{GeV}$ en la región de exclusión de masa de gluinos), se deben a las mejoras introducidas en la performance de identificación, reconstrucción y selección de los objetos físicos, así como de la rigurosa optimización de la estrategia de análisis. Al momento de escribir esta tesis, los resultados finales incluyendo todos los datos de Run-2 están en proceso de aprobación interna de la colaboración para poder hacerse públicos en los próximos meses.

El estado actual del arte de la búsqueda de partículas de Supersimetría en los distintos experimentos del LHC, presenta límites en las masas de las nuevas partí- 
culas cercanas a los $2 \mathrm{TeV}$, lo cual entra en conflicto con la naturalidad de la teoría. Sin embargo, estos límites se basan en una selección especial de modelos, con foco en aquellos que presentan mayor aceptancia en función de sus secciones eficaces, reconstrucción experimental y capacidad a ser diferenciados de procesos del SM. La persistencia de estos resultados pone a la comunidad frente al desafío de encarar nuevas búsquedas guiadas por modelos más sofisticados y más complejas desde el punto de vista experimental. En esta dirección, se presentó al final de la tesis la factibilidad para realizar en un futuro cercano una nueva búsqueda guiada por un modelo con un sector adicional, denominado Stealth SUSY. 


\section{Bibliografía}

[1] ATLAS Collaboration. Search for photonic signatures of gauge-mediated supersymmetry in $8 \mathrm{TeV} p p$ collisions with the ATLAS detector. Phys. Rev. D, 92:072001, 2015.

[2] ATLAS Collaboration. Search for photonic signatures of gauge-mediated supersymmetry in $13 \mathrm{TeV} p p$ collisions with the ATLAS detector. Phys. Rev. D, 97:092006, 2018.

[3] Yu. A. Gol'fand and E. P. Likhtman. Extension of the Algebra of Poincare Group Generators and Violation of p Invariance. JETP Lett., 13:323-326, 1971. [Pisma Zh.Eksp.Teor.Fiz. 13:452-455,1971].

[4] D. V. Volkov and V. P. Akulov. Is the Neutrino a Goldstone Particle? Phys. Lett. B, 46:109-110, 1973.

[5] J. Wess and B. Zumino. Supergauge Transformations in Four-Dimensions. Nucl. Phys. B, 70:39-50, 1974.

[6] J. Wess and B. Zumino. Supergauge Invariant Extension of Quantum Electrodynamics. Nucl. Phys. B, 78:1, 1974.

[7] S. Ferrara and B. Zumino. Supergauge Invariant Yang-Mills Theories. Nucl. Phys. B, 79:413, 1974.

[8] Abdus Salam and J. A. Strathdee. Supersymmetry and Nonabelian Gauges. Phys. Lett. B, 51:353-355, 1974.

[9] Stephen P. Martin. A Supersymmetry primer. 1997. [Adv. Ser. Direct. High Energy Phys.18,1(1998)].

[10] Michael Dine and Willy Fischler. A Phenomenological Model of Particle Physics Based on Supersymmetry. Phys. Lett. B, 110:227, 1982.

[11] Luis Alvarez-Gaume, Mark Claudson, and Mark B. Wise. Low-Energy Supersymmetry. Nucl. Phys. B, 207:96, 1982.

[12] Chiara R. Nappi and Burt A. Ovrut. Supersymmetric Extension of the SU(3) x SU(2) x U(1) Model. Phys. Lett. B, 113:175, 1982.

[13] H. Goldberg. Constraint on the photino mass from cosmology. Phys. Rev. Lett., 50:1419, 1983.

[14] J.R. Ellis, J.S. Hagelin, D.V. Nanopoulos, K.A. Olive, and M. Srednicki. Supersymmetric relics from the big bang. Nucl. Phys. B, 238:453-476, 1984. 
[15] ATLAS Collaboration. Observation of a new particle in the search for the Standard Model Higgs boson with the ATLAS detector at the LHC. Phys. Lett. B, 716:1, 2012.

[16] CMS Collaboration. Observation of a new boson at a mass of $125 \mathrm{GeV}$ with the CMS experiment at the LHC. Phys. Lett. B, 716:30, 2012.

[17] Gordon Kane. Modern Elementary Particle Physics: Explaining and Extending the Standard Model. Cambridge University Press, 2 edition, 2017.

[18] T.D. Lee. Particle Physics and Introduction to Field Theory: Revised and Updated First Edition. Taylor \& Francis, 1981.

[19] D. Griffiths. Introduction to Elementary Particles. Wiley, 2008.

[20] Andrew Purcell. Go on a particle quest at the first CERN webfest. Le premier webfest du CERN se lance à la conquête des particules. (BUL-NA-2012-269. 35/2012):10, Aug 2012.

[21] P. W. Anderson. Plasmons, gauge invariance, and mass. Phys. Rev., 130:439442, Apr 1963.

[22] Yoichiro Nambu. Quasi-particles and gauge invariance in the theory of superconductivity. Phys. Rev., 117:648-663, Feb 1960.

[23] Peter W. Higgs. Broken symmetries and the masses of gauge bosons. Phys. Rev. Lett., 13:508-509, Oct 1964.

[24] F. Englert and R. Brout. Broken symmetry and the mass of gauge vector mesons. Phys. Rev. Lett., 13:321-323, Aug 1964.

[25] G. S. Guralnik, C. R. Hagen, and T. W. B. Kibble. Global conservation laws and massless particles. Phys. Rev. Lett., 13:585-587, Nov 1964.

[26] GERALD S. GURALNIK. The history of the guralnik, hagen and kibble development of the theory of spontaneous symmetry breaking and gauge particles. International Journal of Modern Physics A, 24(14):2601-2627, 2009.

[27] John C. Collins, Davison E. Soper, and George Sterman. Factorization of hard processes in qcd, 2004.

[28] V. N. Gribov and L. N. Lipatov. Deep inelastic e p scattering in perturbation theory. Sov. J. Nucl. Phys., 15:438-450, 1972. [Yad. Fiz.15,781(1972)].

[29] Guido Altarelli and G. Parisi. Asymptotic Freedom in Parton Language. Nucl. Phys., B126:298-318, 1977.

[30] Yuri L. Dokshitzer. Calculation of the Structure Functions for Deep Inelastic Scattering and e+ e- Annihilation by Perturbation Theory in Quantum Chromodynamics. Sov. Phys. JETP, 46:641-653, 1977. [Zh. Eksp. Teor. Fiz.73,1216(1977)]. 
[31] James Botts, Jorge G. Morfin, Joseph F. Owens, Jianwei Qiu, Wu-Ki Tung, and Harry Weerts. Cteq parton distributions and flavor dependence of sea quarks. Physics Letters B, 304(1-2):159-166, Apr 1993.

[32] A. D. Martin, W. J. Stirling, R. S. Thorne, and G. Watt. Parton distributions for the lhc. The European Physical Journal C, 63(2):189-285, Jul 2009.

[33] A. D. Martin, W. J. Stirling, R. S. Thorne, and G. Watt. Uncertainties on $\alpha_{s}$ in global pdf analyses and implications for predicted hadronic cross sections. The European Physical Journal C, 64(4):653-680, Oct 2009.

[34] A. D. Martin, W. J. Stirling, R. S. Thorne, and G. Watt. Heavy-quark mass dependence in global pdf analyses and 3- and 4-flavour parton distributions. The European Physical Journal C, 70(1-2):51-72, Oct 2010.

[35] Carrazza S. Deans C. Del Debbio L. Forte S. Guffanti A. Hartland N. Latorre J. Rojo J. et al. Ball R., Bertone V. Parton distributions with lhc data. Nuclear Physics B, 867(2):244-289, Feb 2013.

[36] Stefan Höche. Introduction to parton-shower event generators, 2014.

[37] Stefano Catani, Frank Krauss, Bryan R Webber, and Ralf Kuhn. Qcd matrix elements + parton showers. Journal of High Energy Physics, 2001(11):063-063, Nov 2001.

[38] Frank Krauss. Matrix elements and parton showers in hadronic interactions. Journal of High Energy Physics, 2002(08):015-015, Aug 2002.

[39] Michelangelo L Mangano, Fulvio Piccinini, Antonio D Polosa, Mauro Moretti, and Roberto Pittau. Alpgen, a generator for hard multiparton processes in hadronic collisions. Journal of High Energy Physics, 2003(07):001-001, Jul 2003.

[40] Nathalie Palanque-Delabrouille, Christophe Yèche, Julien Baur, Christophe Magneville, Graziano Rossi, Julien Lesgourgues, Arnaud Borde, Etienne Burtin, Jean-Marc LeGoff, James Rich, and et al. Neutrino masses and cosmology with lyman-alpha forest power spectrum. Journal of Cosmology and Astroparticle Physics, 2015(11):011-011, Nov 2015.

[41] Martín Tripiana. Medida de la sección eficaz de producción de fotones directos aislados en colisiones pp a $\sqrt{s}=7 \mathrm{TeV}$ en el experimento ATLAS, Marzo 2012. Tesis de Doctorado Universidad Nacional de La Plata.

[42] H. et al. Nishino. Search for proton decay via $p \rightarrow e^{+} \pi^{0}$ and $p \rightarrow \mu^{+} \pi^{0}$ in a large water cherenkov detector. Phys. Rev. Lett., 102:141801, Apr 2009.

[43] Stephen P. Martin. A supersymmetry primer. Advanced Series on Directions in High Energy Physics, page 1-98, Jul 1998.

[44] Francisco Alonso. Búsqueda de Supersimetría en eventos con un fotón, jets y energía faltante con el detector ATLAS, Febrero 2016. Tesis de Doctorado Universidad Nacional de La Plata. 
[45] Search for photonic signatures of gauge-mediated supersymmetry in $8 \mathrm{TeV} p p$ collisions with the ATLAS detector. Phys. Rev. D, 92:072001, 2015.

[46] Francisco Anuar Arduh. Búsqueda de supersimetría en procesos con un fotón, electrón/muón y energía perdida en estado final con el detector ATLAS del LHC, Marzo 2018. Tesis de Doctorado Universidad Nacional de La Plata.

[47] ATLAS Collaboration. Search for supersymmetry in a final state containing two photons and missing transverse momentum in $\sqrt{s}=13 \mathrm{TeV} p p$ collisions at the LHC using the ATLAS detector. Eur. Phys. J. C, 76:517, 2016.

[48] JiJi Fan, Matthew Reece, and Joshua T. Ruderman. Stealth supersymmetry. Journal of High Energy Physics, 2011(11), Nov 2011.

[49] JiJi Fan, Matthew Reece, and Joshua T. Ruderman. A stealth supersymmetry sampler. Journal of High Energy Physics, 2012(7), Jul 2012.

[50] Lyndon R Evans and Philip Bryant. LHC Machine. J. Instrum., 3:S08001. $164 \mathrm{p}, 2008$. This report is an abridged version of the LHC Design Report (CERN-2004-003).

[51] LEP design report. CERN, Geneva, 1983. By the LEP Injector Study Group.

[52] G. Aad et al. The ATLAS Experiment at the CERN Large Hadron Collider. JINST, 3:S08003, 2008.

[53] S. Chatrchyan et al. The CMS Experiment at the CERN LHC. JINST, 3:S08004, 2008.

[54] K. Aamodt et al. The ALICE experiment at the CERN LHC. JINST, 3:S08002, 2008.

[55] A. Augusto Alves, Jr. et al. The LHCb Detector at the LHC. JINST, 3:S08005, 2008.

[56] ATLAS Collaboration. The ATLAS Experiment at the CERN Large Hadron Collider. JINST, 3:S08003, 2008.

[57] ATLAS Collaboration. Atlas insertable b-layer technical design report. ATLAS-TDR-19, 2010.

[58] B. Abbott et al. Production and integration of the ATLAS Insertable B-Layer. JINST, 13:T05008, 2018.

[59] ATLAS Collaboration. ATLAS technical coordination: Technical Design Report. Technical Design Report ATLAS. CERN, Geneva, 1999.

[60] H. H. Ten Kate. Superconducting magnet system for the atlas detector at cern. IEEE Transactions on Applied Superconductivity, 9(2):841-846, June 1999.

[61] Vertex Reconstruction Performance of the ATLAS Detector at $\sqrt{s}=13 \mathrm{TeV}$. Technical Report ATL-PHYS-PUB-2015-026, CERN, Geneva, Jul 2015. 
[62] Valerio Rossetti. Performance of the ATLAS Calorimeters and Commissioning for LHC Run-2. Jul 2015.

[63] ATLAS Collaboration. Performance of the ATLAS Trigger System in 2010. Eur. Phys. J. C, 72:1849, 2012.

[64] ATLAS Collaboration. Performance of the ATLAS trigger system in 2015. Eur. Phys. J. C, 77:317, 2017.

[65] R. Achenbach et al. The ATLAS level-1 calorimeter trigger. JINST, 3:P03001, 2008.

[66] Peter Jenni, Marzio Nessi, Markus Nordberg, and Kenway Smith. ATLAS high-level trigger, data-acquisition and controls: Technical Design Report. Technical Design Report ATLAS. CERN, Geneva, 2003.

[67] CERN. The worldwide lhe computing grid.

[68] CERN. ATLAS Computing: technical design report. Technical Design Report ATLAS. Geneva, 2005.

[69] B Lenzi. The Physics Analysis Tools project for the ATLAS experiment. Technical Report ATL-SOFT-PROC-2009-006, CERN, Geneva, Oct 2009.

[70] P Calafiura, W Lavrijsen, C Leggett, M Marino, and D Quarrie. The Athena Control Framework in Production, New Developments and Lessons Learned. 2005.

[71] R. Brun and F. Rademakers. ROOT: An object oriented data analysis framework. Nucl. Instrum. Meth., A389:81-86, 1997.

[72] ATLAS Collaboration. Luminosity determination in $p p$ collisions at $\sqrt{s}=13$ TeV using the ATLAS detector at the LHC. ATLAS-CONF-2019-021, 2019.

[73] G. Avoni et al. The new lucid-2 detector for luminosity measurement and monitoring in atlas. JINST, 13(07):P07017, 2018.

[74] ATLAS Collaboration. Reconstruction of primary vertices at the ATLAS experiment in Run 1 proton-proton collisions at the LHC. Eur. Phys. J. C, 77:332, 2017.

[75] ATLAS Collaboration. Data-Quality Requirements and Event Cleaning for Jets and Missing Transverse Energy Reconstruction with the ATLAS Detector in Proton-Proton Collisions at a Center-of-Mass Energy of $\sqrt{s}=7 \mathrm{TeV}$. ATLAS-CONF-2010-038, 2010.

[76] ATLAS Collaboration. The Pythia 8 A3 tune description of ATLAS minimum bias and inelastic measurements incorporating the Donnachie-Landshoff diffractive model. ATL-PHYS-PUB-2016-017, 2016.

[77] ATLAS Collaboration. The ATLAS Simulation Infrastructure. Eur. Phys. J. C, 70:823, 2010 . 
[78] S. Agostinelli et al. GEANT4: A Simulation toolkit. Nucl. Instrum. Meth. A, 506:250-303, 2003.

[79] ATLAS Collaboration. Electron and photon performance measurements with the ATLAS detector using the 2015-2017 LHC proton-proton collision data. 2019.

[80] ATLAS Collaboration. Topological cell clustering in the ATLAS calorimeters and its performance in LHC Run 1. Eur. Phys. J. C, 77:490, 2017.

[81] ATLAS Collaboration. Improved electron reconstruction in ATLAS using the Gaussian Sum Filter-based model for bremsstrahlung. ATLAS-CONF-2012047, 2012.

[82] ATLAS Collaboration. Electron efficiency measurements with the ATLAS detector using the 2015 LHC proton-proton collision data. ATLAS-CONF2016-024, 2016.

[83] G. Aad, B. Abbott, D.C. Abbott, A. Abed Abud, K. Abeling, D.K. Abhayasinghe, S.H. Abidi, O.S. AbouZeid, N.L. Abraham, H. Abramowicz, and et al. Electron and photon performance measurements with the atlas detector using the 2015-2017 lhc proton-proton collision data. Journal of Instrumentation, 14(12):P12006-P12006, Dec 2019.

[84] Morad Aaboud et al. Measurement of the photon identification efficiencies with the ATLAS detector using LHC Run 2 data collected in 2015 and 2016. Submitted to: Eur. Phys. J., 2018.

[85] Matteo Cacciari and Gavin P. Salam. Pileup subtraction using jet areas. Phys. Lett., B659:119-126, 2008.

[86] ATLAS Collaboration. Muon reconstruction performance of the ATLAS detector in proton-proton collision data at $\sqrt{s}=13 \mathrm{TeV}$. Eur. Phys. J. C, 76:292, 2016.

[87] G. Aad, B. Abbott, J. Abdallah, O. Abdinov, B. Abeloos, R. Aben, M. Abolins, O. S. AbouZeid, N. L. Abraham, and et al. Muon reconstruction performance of the atlas detector in proton-proton collision data at $\sqrt{s}=13$ tev. The European Physical Journal C, 76(5), May 2016.

[88] Matteo Cacciari, Gavin P. Salam, and Gregory Soyez. The Anti- $k_{t}$ jet clustering algorithm. JHEP, 0804:063, 2008.

[89] W. Lampl, S. Laplace, D. Lelas, P. Loch, H. Ma, S. Menke, S. Rajagopalan, D. Rousseau, S Snyder, and G Unal. Calorimeter Clustering Algorithms: Description and Performance. Technical Report ATL-LARG-PUB-2008-002. ATL-COM-LARG-2008-003, CERN, Geneva, Apr 2008.

[90] ATLAS Collaboration. Jet reconstruction and performance using particle flow with the ATLAS Detector. Eur. Phys. J. C, 77:466, 2017. 
[91] ATLAS Collaboration. Jet energy scale measurements and their systematic uncertainties in proton-proton collisions at $\sqrt{s}=13 \mathrm{TeV}$ with the ATLAS detector. Phys. Rev. D, 96:072002, 2017.

[92] ATLAS Collaboration. Performance of pile-up mitigation techniques for jets in $p p$ collisions at $\sqrt{s}=8 \mathrm{TeV}$ using the ATLAS detector. Eur. Phys. J. C, 76:581, 2016.

[93] ATLAS Collaboration. $E_{\mathrm{T}}^{\text {miss }}$ performance in the ATLAS detector using 20152016 LHC pp collisions. ATLAS-CONF-2018-023, 2018.

[94] ATLAS Collaboration. Expected performance of the ATLAS $b$-tagging algorithms in Run-2. ATLAS-PHYS-PUB-2015-022, 2015.

[95] A. Lavrenov. Mv2-algorithm's clones, 2002.

[96] Marie Lanfermann. Deep Learning in Flavour Tagging at the ATLAS experiment. PoS, EPS-HEP2017:764, 2018.

[97] ATLAS Collaboration. Commissioning of the ATLAS b-tagging algorithms using $t \bar{t}$ events in early Run-2 data. ATLAS-PHYS-PUB-2015-039, 2015.

[98] ATLAS Collaboration. Measurements of $b$-jet tagging efficiency with the ATLAS detector using $t \bar{t}$ events at $\sqrt{s}=13 \mathrm{TeV}$. 2018.

[99] ATLAS Collaboration. Expected performance of missing transverse momentum reconstruction for the ATLAS detector at $\sqrt{s}=13 \mathrm{TeV}$. ATLAS-PHYSPUB-2015-023, 2015.

[100] https://twiki.cern.ch/twiki/bin/view/AtlasProtected/ EtmissRecommendationsRe120p7.

[101] ATLAS Collaboration. Performance of Missing Transverse Momentum Reconstruction in ATLAS studied in Proton-Proton Collisions recorded in 2012 at $\sqrt{s}=8 \mathrm{TeV}$. ATLAS-CONF-2013-082, 2013.

[102] ATLAS Collaboration. Jet energy measurement with the ATLAS detector in proton-proton collisions at $\sqrt{s}=7 \mathrm{TeV}$. Eur. Phys. J. C, 73:2304, 2013.

[103] ATLAS Collaboration. Performance of electron and photon triggers in ATLAS during LHC Run 2. Eur. Phys. J. C, 2020.

[104] Rudolf Erich Raspe. Las sorprendentes aventuras del Barón Münchhausen. 1785.

[105] Bradley Efron. Bootstrap methods: another look at the jackknife. Springer, 1979.

[106] ATLAS Collaboration. Performance of the ATLAS Trigger System in 2010. Eur. Phys. J. C, 72:1849, 2012.

[107] ATLAS Collaboration. Performance of the ATLAS Electron and Photon Trigger in $p p$ Collisions at $\sqrt{s}=7 \mathrm{TeV}$ in 2011. ATLAS-CONF-2012-048, 2012. 
[108] Morad Aaboud et al. Performance of the ATLAS Trigger System in 2015. Eur. Phys. J., C77(5):317, 2017.

[109] Fernando Monticelli. Producción de $\mathrm{J} / \psi \rightarrow e^{+} e^{-}$en ATLAS a $\sqrt{s}=7 \mathrm{TeV}$. Determinación de la eficiencia del Trigger de e y $\gamma$ con $45 \mathrm{pb}^{-1}$ de datos para la búsqueda del Higgs en el canal $\mathrm{H} \rightarrow \gamma \gamma$, Febrero 2011. Tesis de Doctorado Universidad Nacional de La Plata.

[110] Maria Josefina Alconada Verzini. Search for the $125 \mathrm{GeV}$ Higgs Boson at 13 $\mathrm{TeV}$ in diboson decay channels by the ATLAS collaboration. Abril 2016. Tesis de Doctorado Universidad Nacional de La Plata.

[111] ATLAS Collaboration. Measurement of the inclusive isolated prompt photon cross section in $p p$ collisions at $\sqrt{s}=8 \mathrm{TeV}$ with the ATLAS detector. JHEP, 08:005, 2016.

[112] ATLAS Collaboration. Search for new phenomena with photon + jet events in proton-proton collisions at $\sqrt{s}=13 \mathrm{TeV}$ with the ATLAS detector. JHEP, 03:041, 2016.

[113] Search for new phenomena in final states with large jet multiplicities and missing transverse momentum using $\sqrt{s}=13 \mathrm{TeV}$ proton-proton collisions recorded by ATLAS in Run 2 of the LHC. Technical Report ATLAS-CONF2020-002, CERN, Geneva, Feb 2020.

[114] ATLAS Collaboration. Jet global sequential corrections with the ATLAS detector in proton-proton collisions at $\sqrt{s}=8 \mathrm{TeV}$. ATLAS-CONF-2015-002, 2015.

[115] ATLAS Collaboration. Tagging and suppression of pileup jets with the ATLAS detector. ATLAS-CONF-2014-018, 2014.

[116] ATLAS Collaboration. Selection of jets produced in $13 \mathrm{TeV}$ proton-proton collisions with the ATLAS detector. ATLAS-CONF-2015-029, 2015.

[117] https://indico.cern.ch/event/587852/.

[118] D et al. Adams. Recommendations of the Physics Objects and Analysis Harmonisation Study Groups 2014. Technical Report ATL-PHYS-INT-2014-018, CERN, Geneva, Jul 2014.

[119] D et al. Adams. Recommendations of the Physics Objects and Analysis Harmonisation Study Groups 2014. Technical Report ATL-COM-PHYS-2014-451, CERN, Geneva, May 2014.

[120] ATLAS Collaboration. Search for supersymmetry in a final state containing two photons and missing transverse momentum in $\sqrt{s}=13 \mathrm{TeV} p p$ collisions at the LHC using the ATLAS detector. 2016.

[121] https://twiki.cern.ch/twiki/bin/view/AtlasProtected/

SusyObjectDefinitionsr2013TeV\#Overlap_Removals. 
[122] Baptiste Abeloos et al. Search for squarks and gluinos with the ATLAS detector in final states with jets and missing transverse momentum at $\sqrt{s}=13 \mathrm{TeV}$ : supporting documentation for Moriond 2017. Technical Report ATL-COMPHYS-2016-1518, CERN, Geneva, Oct 2016.

[123] Georges Aad et al. Combined Measurement of the Higgs Boson Mass in $p p$ Collisions at $\sqrt{s}=7$ and $8 \mathrm{TeV}$ with the ATLAS and CMS Experiments. Phys. Rev. Lett., 114:191803, 2015.

[124] Nathaniel Craig, Daniel Green, and Andrey Katz. (De)Constructing a Natural and Flavorful Supersymmetric Standard Model. JHEP, 1107:045, 2011.

[125] Roberto Auzzi, Amit Giveon, and Sven Bjarke Gudnason. Flavor of quiver-like realizations of effective supersymmetry. JHEP, 1202:069, 2012.

[126] Csaba Csaki, Lisa Randall, and John Terning. Light Stops from Seiberg Duality. Phys.Rev., D86:075009, 2012.

[127] Grant Larsen, Yasunori Nomura, and Hannes L.L. Roberts. Supersymmetry with Light Stops. JHEP, 1206:032, 2012.

[128] Nathaniel Craig, Savas Dimopoulos, and Tony Gherghetta. Split families unified. JHEP, 1204:116, 2012.

[129] W. Beenakker, R. Hopker, M. Spira, and P.M. Zerwas. Squark and gluino production at hadron colliders. Nucl. Phys. B, 492:51-103, 1997.

[130] A. Kulesza and L. Motyka. Threshold resummation for squark-antisquark and gluino-pair production at the LHC. Phys. Rev. Lett., 102:111802, 2009.

[131] A. Kulesza and L. Motyka. Soft gluon resummation for the production of gluino-gluino and squark-antisquark pairs at the LHC. Phys. Rev. D, 80:095004, 2009.

[132] Wim Beenakker, Silja Brensing, Michael Kramer, Anna Kulesza, Eric Laenen, et al. Soft-gluon resummation for squark and gluino hadroproduction. JHEP, 0912:041, 2009.

[133] W. Beenakker, S. Brensing, M. Kramer, A. Kulesza, E. Laenen, et al. Squark and gluino hadroproduction. Int. J. Mod. Phys. A, 26:2637-2664, 2011.

[134] Christoph Borschensky, Michael Kramer, Anna Kulesza, Michelangelo Mangano, Sanjay Padhi, Tilman Plehn, and Xavier Portell. Squark and gluino production cross sections in pp collisions at $\sqrt{s}=13,14,33$ and $100 \mathrm{TeV}$. Eur. Phys. J. C, 74:3174, 2014.

[135] Abdelhak Djouadi, Jean-Loïc Kneur, and Gilbert Moultaka. Suspect: A fortran code for the supersymmetric and higgs particle spectrum in the $\{\mathrm{MSSM}\}$. Computer Physics Communications, 176(6):426 - 455, 2007.

[136] M. Muhlleitner. SDECAY: A Fortran code for SUSY particle decays in the MSSM. Acta Phys.Polon., B35:2753-2766, 2004. 
[137] A. Djouadi, J. Kalinowski, and M. Spira. HDECAY: A Program for Higgs boson decays in the standard model and its supersymmetric extension. Comput.Phys.Commun., 108:56-74, 1998.

[138] A. Djouadi, M.M. Muhlleitner, and M. Spira. Decays of supersymmetric particles: The Program SUSY-HIT (SUspect-SdecaY-Hdecay-InTerface). Acta Phys.Polon., B38:635-644, 2007.

[139] Elzbieta Richter-Was, D Froidevaux, and Luc Poggioli. ATLFAST 2.0 a fast simulation package for ATLAS. Technical Report ATL-PHYS-98-131, CERN, Geneva, Nov 1998.

[140] T. Gleisberg et al. Event Generation With SHERPA 1.1. JHEP, 0910:007, 2009.

[141] Steffen Schumann and Frank Krauss. A Parton shower algorithm based on Catani-Seymour dipole factorisation. JHEP, 0803:038, 2008.

[142] Stefan Hoeche, Frank Krauss, Marek Schonherr, and Frank Siegert. QCD matrix elements + parton showers: The NLO case. JHEP, 1304:027, 2013.

[143] Hung-Liang Lai, Marco Guzzi, Joey Huston, Zhao Li, Pavel M. Nadolsky, Jon Pumplin, and C. P. Yuan. New parton distributions for collider physics. Phys. Rev. D, 82:074024, 2010.

[144] J. Alwall, R. Frederix, S. Frixione, V. Hirschi, F. Maltoni, O. Mattelaer, H. S. Shao, T. Stelzer, P. Torrielli, and M. Zaro. The automated computation of treelevel and next-to-leading order differential cross sections, and their matching to parton shower simulations. JHEP, 1407:079, 2014.

[145] Torbjörn Sjöstrand, Stefan Ask, Jesper R. Christiansen, Richard Corke, Nishita Desai, Philip Ilten, Stephen Mrenna, Stefan Prestel, Christine O. Rasmussen, and Peter Z. Skands. An Introduction to PYTHIA 8.2. Comput. Phys. Commun., 191:159-177, 2015.

[146] ATLAS Collaboration. Measurement of the inclusive isolated prompt photon cross section in $p p$ collisions at $\sqrt{s}=7 \mathrm{TeV}$ with the ATLAS detector. Phys. Rev. D, 83:052005, 2011.

[147] Francisco Alonso, Francisco Anuar Arduh, Joaquin Hoya, Maria-Teresa Dova, Hernan Wahlberg, and Gonzalo Enrique Orellana. Search for supersymmetry in events with photons, jets and missing transverse momentum with $36 \mathrm{fb}^{-1}$ of data at $13 \mathrm{TeV}$. Technical Report ATL-COM-PHYS-2016-1662, CERN, Geneva, Nov 2016.

[148] Leonard Aubry et al. Search for new phenomena with the ATLAS detector in monophoton events from proton-proton collisions at sqrts $=13 \mathrm{TeV}$ collected in 2015 and 2016. Technical Report ATL-COM-PHYS-2016-1626, CERN, Geneva, Nov 2016.

[149] H Abreu et al. Purity Estimates for the Inclusive Isolated Photons. Technical Report ATL-PHYS-INT-2011-015, CERN, Geneva, Mar 2011. 
[150] Gonzalo Enrique Orellana. Estudios de fondos de electrones reconstruidos como fotones en búsquedas de supersimetría con el detector ATLAS, Marzo 2017. Tesis de Licenciatura Universidad Nacional de La Plata.

[151] Jerzy Neyman, Egon Sharpe Pearson, and Karl Pearson. Ix. on the problem of the most efficient tests of statistical hypotheses. Philosophical Transactions of the Royal Society of London., 231(694-706):289-337, 1933.

[152] ATLAS Collaboration. Improved luminosity determination in $p p$ collisions at $\sqrt{s}=7 \mathrm{TeV}$ using the ATLAS detector at the LHC. Eur. Phys. J. C, 73:2518, 2013 .

[153] ATLAS Collaboration. Measurement of the photon identification efficiencies with the ATLAS detector using LHC Run-1 data. 2016.

[154] ATLAS Collaboration. Photon identification in 2015 ATLAS data. ATLPHYS-PUB-2016-014, 2016.

[155] https://twiki.cern.ch/twiki/bin/view/AtlasProtected/ EGammaCalibrationRun2.

[156] Louis Fayard, Goudet, et al. Energy calibration prerecommendation for Run 2. Technical Report ATL-COM-PHYS-2015-1300, CERN, Geneva, Oct 2015.

[157] ATLAS Collaboration. Electron and photon energy calibration with the ATLAS detector using LHC Run 1 data. Eur. Phys. J. C, 74:3071, 2014.

[158] Xabier Sebastián Anduaga. Búsqueda de dimensiones espaciales extras. Estudio del estado final de dos fotones en el experimento ATLAS, Marzo 2012. Tesis de Doctorado Universidad Nacional de La Plata.

[159] ATLAS Collaboration. Electron efficiency measurements with the ATLAS detector using the 2012 LHC proton-proton collision data. ATLAS-CONF2014-032, 2014.

[160] https://twiki.cern.ch/twiki/bin/viewauth/AtlasProtected/ EfficiencyMeasurements.

[161] https://twiki.cern.ch/twiki/bin/view/AtlasProtected/ MuonPerformance.

[162] ATLAS Collaboration. Monte Carlo Calibration and Combination of In-situ Measurements of Jet Energy Scale, Jet Energy Resolution and Jet Mass in ATLAS. ATLAS-CONF-2015-037, 2015.

[163] https://twiki.cern.ch/twiki/bin/viewauth/AtlasProtected/ EtmissRecommendationsRel20p7.

[164] Michael Kramer, Anna Kulesza, Robin van der Leeuw, Michelangelo Mangano, Sanjay Padhi, et al. Supersymmetry production cross sections in pp collisions at $\sqrt{s}=7 \mathrm{TeV} .2012$.

[165] Jon Butterworth et al. PDF4LHC recommendations for LHC Run II. J. Phys. G, 43:023001, 2016. 
[166] https://twiki.cern.ch/twiki/bin/view/AtlasProtected/ PdfRecommendations.

[167] Sayipjamal Dulat, Tie Jiun Hou, Jun Gao, Marco Guzzi, Joey Huston, Pavel Nadolsky, Jon Pumplin, Carl Schmidt, Daniel Stump, and C. P. Yuan. New parton distribution functions from a global analysis of quantum chromodynamics, 2015.

[168] L. A. Harland-Lang, A. D. Martin, P. Motylinski, and R. S. Thorne. Parton distributions in the lhc era: Mmht 2014 pdfs, 2014.

[169] M. Baak, G. J. Besjes, D. Cote, A. Koutsman, J. Lorenz, and D. Short. Histfitter software framework for statistical data analysis, 2014.

[170] Alexander L. Read. Presentation of search results: The $C L_{s}$ technique. $J$. Phys. G, 28:2693-2704, 2002.

[171] ATLAS Collaboration. ATLAS detector and physics performance: Technical Design Report, 2. Technical Design Report ATLAS. CERN, Geneva, 1999.

[172] Object-based missing transverse momentum significance in the ATLAS detector. Technical Report ATLAS-CONF-2018-038, CERN, Geneva, Jul 2018.

[173] Search for massive supersymmetric particles in multi-jet final states produced in pp collisions at $\sqrt{s}=13 \mathrm{TeV}$ using the ATLAS detector at the LHC. Technical Report ATLAS-CONF-2016-057, CERN, Geneva, Aug 2016. 


\section{Agradecimientos}

En primer lugar quiero agradecer a Tere y Hernán, no sólo por la oportunidad que me dieron para trabajar con ellos en el increíble mundo del LHC, sino también por todo el tiempo, la dedicación, la confianza y todos los conocimientos que me han transmitido a lo largo de estos años.

A todo el extraordinario grupo de HEP de La Plata con el que aprendo todos los días!! En especial a Fer, Fran y Gonza por las horas compartidas trabajando juntos y la incalculable ayuda que me dieron. Un verdadero placer trabajar así...

Also want to thank the amazing people I met at CERN and Geneva for all the learning but also for all the friends I made!! An special remark to Anna for the opportunity to work with her at the UNIGE.

Al CONICET y a la UNLP por su apoyo, y a todos lo que trabajan cada día por hacer ciencia de calidad para el país y los que participan de algo tan importante como lo es la educación pública, libre y gratuita.

A mis amigos por estar siempre, aún estando lejos..

A mi familia, por el apoyo incondicional.

Y a Majo, por ser mi compañera siempre. 